Meeting Report

\title{
Abstracts of the 10th Asia-Pacific Conference on Clinical Nutrition ${ }^{+}$
}

\author{
Jonathan Buckley 1,*, Malcolm Riley ${ }^{2}$, Lisa Wood ${ }^{3}$, Sheila Skeaff ${ }^{4}$ and Manny Noakes ${ }^{2}$ \\ 1 School of Health Sciences, University of South Australia, Adelaide, South Australia 5001, Australia \\ 2 CSIRO Health and Biosecurity, Adelaide, South Australia 5001, Australia; malcolm.riley@csiro.au (M.R.); \\ manny.noakes@csiro.au (M.N.) \\ 3 School of Biomedical Sciences and Pharmacy, University of Newcastle, Callaghan, New South Wales 2308, \\ Australia; lisa.wood@newcastle.edu.au \\ 4 Department of Human Nutrition, University of Otago, Dunedin 9054, New Zealand; \\ sheila.skeaff@otago.ac.nz \\ * Correspondence: jon.buckley@unisa.edu.au; Tel.: +61-8-83021853
}

Received: 16 April 2018; Accepted: 20 April 2018; Published: 9 August 2018

\begin{abstract}
The Asia-Pacific Conference on Clinical Nutrition is a biennial conference held within the Asia-Pacific region. The 2017 meeting was a joint meeting of the Asia-Pacific Society of Clinical Nutrition, the Nutrition Society of Australia and the Nutrition Society of New Zealand. The meeting was hosted by CSIRO Health and Biosecurity in collaboration with the University of South Australia, the University of Adelaide, Flinders University and the South Australian Health and Medical Research Institute. The theme of the meeting was Nutrition Solutions for a Changing World. Four hundred and thirty-eight registrants attended the conference and 432 papers were presented. This issue presents the proceedings of this meeting in the form of abstracts for each paper that was presented at the conference.
\end{abstract}

Keywords: nutrients, disease, vitamins, minerals, macronutrients, micronutrients

\section{Plenary Presentations}

\subsection{The Age of Food}

\section{J. H. J. Cribb}

Julian Cribb \& Associates, Australia

Dramatic evolution in the global diet, cuisine and consumer preferences will, over the coming decades, change the foods we eat more profoundly than at any time in history. Emerging scarcities of land, water, oil, nutrients, fish and climate instability along with concerns about health and sustainability signal a major shift in the world food production paradigm from extensive, open-air and rural to intensive, indoors, urban and high tech. Remarkable new food and nutritional opportunities await us in this, The Age of Food.

\subsection{Nutrition Solutions for the Asia-Pacific Region}

\section{R. A. Gibson}

Foods Science and Nutrition, School of Agriculture Food and Wine, University of Adelaide, Adelaide, SA 5005, Australia

Nutritional needs in the Asia-Pacific Region range from areas with severe nutritional deficiencies to problems resulting from over-nutrition. Standards of living range from high-rise 
apartments in some of the most modern cities in the world to humble villages with little or no access to modern amenities. One issue unites us all in the Region-vast distances separated by oceans, forests, deserts sometimes poorly served by transportation. Addressing the nutritional needs of all peoples in the region is a priority. Like all areas, the nutritional needs of peoples in the Asia-Pacific Region have to be assessed using accurate tools, results interpreted according to latest knowledge, appropriate interventions developed in collaboration with locals and then well designed and well powered studies conducted to confirm the effectiveness of the intervention. This has to be followed by the hardest task - to develop low cost, socially acceptable interventions for the population. There are increasing tools available for the rapid determination of nutrient status and scientists can no longer hide behind the tyranny of distance and the need for complex tools of analysis as an excuse for doing nothing.

A major advance in the collection of biological fluids has been developed using dried spots on paper and other matrixes treated with protective chemicals. Similar to the ubiquitous Guthrie cards, these collection devices allow the storage and transport of biological samples without the need for freezers and can be transported by post. When connected to LC-MSMS technology via auto-samplers, huge numbers of nutrients can be monitored in a single sample. New data suggest that dried samples are even more stable than frozen samples since there is no aqueous medium to allow for enzymatic reactions. Examples of larger-scale clinical trials conducted across vast distances will be given.

\subsection{Food with Anti-Diabetes Activity}

\section{D. $\mathrm{Li}$}

Institute of Nutrition \& Health, Qingdao University, Qingdao 266071, China

Type 2 diabetes (T2D) continues to grow globally, especially in developing countries, such as China and India. As we all know that diet plays an important role in human health. Epidemiological, observational, prospective cohort studies and randomized controlled trails revealed that dietary intake is closely associated with T2D. Different dietary patterns have different effect on risk of T2D. Mediterranean diet, Dietary Approaches to Stop Hypertension (DASH) and Alternative Healthy Eating Index (AHEI) are significantly negatively associated with incident of T2D. Compared with higher glycemic index (GI) and glycemic load (GL) foods, lower GI \& GL foods are associated with decreased rick of T2D. Whole grain, oats and oat bran, psyllium, wheat germ, soy bean and legume are associated with reduced risk of T2D compared with fine carbohydrate. Fruits and vegetables are protective factors of T2D. Total protein and animal protein, and protein from red meat and processed meat are risk factors of T2D. Plant protein (mainly from soy), and protein from dairy and dairy products are the protective factors of T2D. Protein from egg and fish intake are not associated with a decreased risk of T2D. Effect of lipids/fats and fatty acids on T2D risk is much more complicated. Increased dietary intake of total fat and reduced carbohydrate is associated with lower risk of T2D in Western populations, however, this is not a case in Asians. N-3 PUFA have beneficial effects in relation to increased insulin sensitivity in Asians, however, n-3 PUFA may have unfavourable effects on T2D in Caucasians.

\subsection{Nutrition Solutions for Pregnant Women and Their Babies}

\section{Makrides}

South Australian Health and Medical Research Institute, Adelaide 5001, Australia

Better understanding of the essential roles that good nutrition plays in setting the foundations for a healthy start to life has already resulted in impressive improvements in perinatal and longerterm health outcomes. This presentation will highlight two contemporary examples requiring different implementation strategies to maximise health outcomes for mothers and their babies. The first relates to how and when allergenic solid foods are introduced to infants as a strategy to minimise the occurrence of food allergies in early life. Intervention studies and the associated systematic review indicate that allergenic foods such as eggs and peanuts should be regularly included into an infant's 
diet from 6-12 months of age. Appropriate implementation strategies require broad, harmonised, population-wide dissemination. On the other hand, the second example requires a targeted approach because there is a more sensitive consideration of benefit and risk. Supplementing pregnant women with omega-3 long chain fatty acid increases mean gestation length with commensurate decreases in early preterm birth $<34$ weeks and increases in prolonged gestation $>42$ weeks. This pattern of increased length of gestation is also associated with an increase in mean birth weight, a reduction in the frequency of low birth weight and an increase in the frequency of large for gestational age. Prevention of early preterm birth is a global priority and implementation strategies are needed to identify and treat women most likely to benefit from omega-3 LCPUFA without increasing the risks associated with prolonged gestation beyond term.

\subsection{Optimizing Exercise Adaptations in Gold Medal Athletes and Mere Mortals}

\section{S. M. Phillips}

Department of Kinesiology, McMaster University, Hamilton, ON L8S 4L8, Canada

Protein is often an afterthought for some athletes and persons that are physically active. Emerging evidence suggests, however, that protein is not merely a substrate to provide building blocks for proteins, but that various amino acids are also molecular triggers for adaptive processes in skeletal muscle. In this session, the mechanisms underpinning protein turnover in skeletal muscle will be discussed that are critical in optimizing the adaptive responses to exercise. Not only will the focus be on athletes but mere mortals, of all ages. Discussion will focus on understanding protein requirements versus optimal recommendations in exercising persons. The role of protein quality, timing relative to exercise, and age will be variables highlighted that play a crucial role in the promotion of turnover. The most recent analysis of protein's role in promotion of muscle adaptation will be discussed as well as the role of individual amino acids and their derivatives in promoting gains in lean mass. With an increasingly large number of athletes, and casual exercisers, competing at a masters level (and beyond), there will also be a focus on aging. The ostensible downsides of consuming 'too much' protein will also be a topic of discussion.

\subsection{The "One Road One Belt" Initiative and Important Opportunities for International Collaboration in the} Nutrition and Health Fields

Y. Wang ${ }^{1,2}$

1 Global Health Institute, Xi' an Jiaotong University, Xi' an 710049, China

2 Department of Nutrition and Health Sciences College of Health, Ball State University, Muncie, IN 47306, USA

The Silk Road Economic Belt and the 21st-century Maritime Silk Road, also known as the "One Belt, One Road" or the "Belt and Road" Initiative is an ambitious development strategy and framework, proposed by China that focuses on connectivity and cooperation among countries primarily between China and other countries worldwide, which include 66 countries at present.

As the second largest economy in the world, with her population accounting for about $1 / 5$ of the global population, and with China's important and growing international influence, the strategy underlines China's push to take a bigger role in global affairs, including its support and promotion of global collaboration in areas such as trade, research, and public health issues. While the coverage area of the initiative initially is primarily Asia and Europe, Oceania, East Africa and America are also being included. The anticipated cumulative investment over an indefinite timescale is variously put at US $\$ 4$ trillion or US $\$ 8$ trillion. It is estimated that China's investment will be far more than the wellknown US Marshall Plan (e.g., it is stated, "The One Belt One Road Project, in terms of its size, could be multiple times larger and more ambitious than the Marshall Plan or the European enlargement, see below link/article"). China is making major investments, including support for collaboration in joint research and training programs related to health and international aids. 
In China, over the past year, a series of efforts and initiatives have been made to promote global health related research and collaboration, for example, many universities have developed new global health programs, the China CDC established a new Global Health Center in summer 2016, and a new Global Heath Section under the Chinese Preventive Medicine Association was established in fall 2016. The University Alliance of the Silk Road (UASR), a university alliance centered at Xi'an Jiaotong University, aims to support the One Belt, One Road initiative with research and engineering, and to foster understanding and academic exchange. At present, the network includes about 140 universities from about 38 countries and regions worldwide. The UASR aims to support training, research, policy, cross-cultural understanding, and medical service. In September 2016, Xi'an Jiaotong University established an ambitious interdisciplinary cross-campus Global Health Institute, to help foster future research and collaboration regarding health-related topics in China and abroad. The Chair of the proposed session, Prof Youfa Wang is the Founding Dean of the Global Health Institute. It is projected that the One Belt, One Road" initiative will provide many great future opportunities for related international collaborations to address health related issues, while nutrition will be an important part of it.

\subsection{Nutrition and Inflammation}

L. G. Wood 1,2

1 School of Biomedical Science and Pharmacy, University of Newcastle, Callaghan, NSW 2308, Australia

2 Hunter Medical Research Institute, Newcastle, NSW, Australia

21st century dietary habits have underpinned the development of many chronic inflammatory diseases that have now reached epidemic proportions, such as diabetes, cardiovascular disease and some cancers. Modern diets have contributed to this problem by providing fuel for inflammation, with regular consumption of processed and 'fast' foods and low intake of fruit, vegetables and whole grains. This eating pattern promotes obesity and leads to excessive saturated fat and refined carbohydrate intake, combined with inadequate fibre and antioxidant intake, thus creating a proinflammatory environment. Obesity induces a state of chronic, low-grade inflammation, due to the changes in adipose tissue that occur with weight gain and nutrient excess. Saturated free fatty acids, cholesterol and cellular debris left after adipocyte apoptosis, activate innate immune receptors such as toll-like receptor 4 (TLR4), leading to assembly of the NLRP3 inflammasome, macrophage recruitment and polarisation and secretion of pro-inflammatory cytokines. Conversely, a low intake of soluble fibre leads to suppression of beneficial anti-inflammatory mechanisms, such as free fatty acid receptor 2/3 (FFAR2/3) activation and histone deacetylase (HDAC) inhibition. Similarly, with a low intake of antioxidants such as vitamin C, vitamin E, carotenoids and polyphenols, NF- $k B$ activity and production of pro-inflammatory cytokines is enhanced. In our laboratory, we have generated evidence from human and experimental models of asthma, demonstrating that these mechanisms contribute to the development of airway inflammation and the clinical expression of asthma. Evidence suggests that interventions targeting both the quantity and quality of food consumed are needed.

\subsection{The Future of Nutrition}

\section{Noakes}

\section{CSIRO Health \& Biosecurity, Adelaide 5000, Australia}

Self health and wellness and direct to consumer wellness products and services are a burgeoning global industry, in part fueled by our increasing aging population wishing to maintain and optimize their health and reduce morbidity. The global wellness industry is estimated as a \$3.4 trillion market, or 3.4 times larger than the worldwide pharmaceutical industry. Within that sector, preventive and personalised services, nutrition and weight loss, complementary medicine and nutraceuticals encompass almost one third of this economic value. While the health system is under economic pressure, there is an opportunity for gobal food and healthcare sectors to become more effective as 
well as to be major export industries and make a positive contribution to the economy. Nutrition has a key role to play in these areas. The technological revolution is transforming both nutrition science as well as communication. We need not only to keep pace and adapt, but we also need new solutions for nutrition to be relevant in this environment.

Food, nutraceuticals and pharmaceuticals are being purchased more and more outside the conventional means of the supermarket and pharmacy-purchased online from anywhere in the world. Genetic information and services are proliferating and costs their costs are declining. Nutrition, diet and lifestyle are not independent from these developments and we need to understand and embrace the new science of precision health which allows the individual to be more empowered and to have more precise information about how to best manage their health and wellbeing.

\section{Regular Abstracts}

\subsection{Dietary Inflammatory Index in Relation to Sub-Clinical Atherosclerosis and Atherosclerotic Vascular Disease Mortality in Older Women}

N. P. Bondonno ${ }^{1, *}$, J. R. Lewis ${ }^{1}$, L. C. Blekkenhorst ${ }^{1}$, N. Shivappa ${ }^{2}$, R. J. Woodman ${ }^{3}$, C. P. Bondonno ${ }^{1}$, N. C. Ward ${ }^{1}$, J. R. Hébert ${ }^{2}$, P. L. Thompson ${ }^{4}$, R. L. Prince ${ }^{1}$ and J.M. Hodgson ${ }^{1}$

1 School of Medicine, University of Western Australia, Perth, WA 6009, Australia

2 Arnold School of Public Health, University of South Carolina, Columbia, SC 29208, USA

3 School of Public Health, Flinders University of South Australia, Adelaide, SA 5042, Australia

4 Department of Cardiovascular Medicine, Sir Charles Gairdner Hospital, Perth, WA 6009, Australia

* Corresponding Author

Arterial wall thickening, stimulated by low-grade systemic inflammation, underlies many cardiovascular events. As diet is a significant moderator of systemic inflammation, the dietary inflammatory index (DII ${ }^{\mathrm{TM}}$ ) has recently been devised to assess the overall inflammatory potential of an individual's diet. The primary objective of this study was to assess the association of the DII with common carotid artery intima-media thickness (CCA-IMT) and carotid plaques. Additionally, we assessed the relationship of DII score with atherosclerotic vascular disease (ASVD)-related mortality, ischemic cerebrovascular disease (CVA)-related mortality and ischemic heart disease (IHD)-related mortality. The study was conducted in Western Australian women aged over 70 years $(n=1304)$. Dietary data were used to calculate a DII score for each individual. In multivariable-adjusted models, DII scores were associated with subclinical atherosclerosis: a 1 SD (2.13 unit) higher DII score was associated with a $0.013 \mathrm{~mm}$ higher mean CCA-IMT $(p=0.016)$ and a $0.016 \mathrm{~mm}$ higher maximum CCA-IMT $(p=0.008)$, measured at 36 months. There were 269 deaths during follow-up. High DII scores were positively associated with ASVD-related death [per SD, HR: 1.36, 95\% CI: 1.15, 1.60], CVA-related death [per SD, HR: 1.30, 95\% CI: 1.00, 1.69], and IHD-related death [per SD, HR: 1.40, $95 \%$ CI: 1.13, 1.75]. These results support the hypothesis that a pro-inflammatory diet increases systemic inflammation leading to development and progression of atherosclerosis and eventual ASVD-related death.

\subsection{Nitrate-Independent Blood Pressure Lowering Effect of Beetroot Juice: A Meta-Analysis of Current Data}

Zahra Bahadoran, Parvin Mirmiran, Fereidoun Azizi, and Asghar Ghasemi *

Research Institute for Endocrine Sciences, Shahid Beheshti University of Medical Sciences, Tehran 1983963113, Iran

* Corresponding Author

Objectives: The aim of this systematic review and meta-analysis was to clarify several aspects of beetroot juice supplementation on systolic (SBP) and diastolic blood pressure (DBP). Methods: Metaanalysis of randomized clinical trials investigating the potential effect of beetroot juice on resting blood pressures in humans. Results: Nineteen eligible trials included in the analyses were conducted between 2009 and 2016 and included a total of 43 intervention $(n=598)$ and 43 control $(n=598)$ 
groups. Overall, SBP $(-3.55 \mathrm{mmHg}, 95 \% \mathrm{CI}=-4.55,-2.54)$ and DBP $(-1.32 \mathrm{mmHg}, 95 \% \mathrm{CI}=-1.97$, $-0.68)$ were significantly lower in beetroot supplemented groups compared to controls. Mean difference of SBP between beetroot supplemented groups and controls was greater in the case of longer compared to shorter ( $\geq 14$ vs. $<14$ d) study duration $(-5.11$ vs. $-2.67 \mathrm{mmHg}$ ), and highest compared to the lowest (500 vs. 70 and $140 \mathrm{~mL} / \mathrm{d}$ ) dose of beetroot juice $(-4.78 \mathrm{vs}$. $-2.37 \mathrm{mmHg}$ ). A positive correlation between beetroot juice doses and mean differences of blood pressures, and in contrast, a smaller effect size of blood pressures was observed following supplementation with higher $\mathrm{NO}_{3}$ doses ( $\mathrm{mg} / 100 \mathrm{~mL}$ of beetroot juice). A weak effect was observed in meta-analysis of trials that used $\mathrm{NO}_{3}$-depleted beetroot juice as placebo compared to other interventions $(-2.10$ vs. -4.58 $\mathrm{mmHg}$ for SBP, and -0.71 vs. $-2.10 \mathrm{mmHg}$ for DBP). Conclusion: Our findings, however, were a further support for previous reports, suggesting a different paradigm regarding a dose-dependent relationship between inorganic $\mathrm{NO}_{3}$ and its hypotensive effects; our findings also highlighted potential $\mathrm{NO}_{3}$-independent blood pressure lowering effects of beetroot juice.

\subsection{Dietary Nitrate Consumption and Risk of Coronary Heart Disease in Women from the Nurses' Health Study}

J. Jackson 1, G. Zong 2, L. MacDonald-Wicks 1, A.J. Patterson 1, W. C. Willett2,3,4, E. B. Rimm 2,3,4, J. E. Manson ${ }^{4,5}$ and M. McEvoy ${ }^{6}$

1 Priority Research Centre in Physical Activity and Nutrition, University of Newcastle, Callaghan, NSW 2308, Australia

2 Department of Nutrition, Harvard T.H. Chan School of Public Health, Boston, MA 02115, USA

3 Channing Division of Network Medicine, Department of Medicine Brigham and Women's Hospital and Harvard Medical School, Boston, MA 02115, USA

4 Department of Epidemiology, Harvard T.H. Chan School of Public Health, Boston, MA 02115, USA

5 Division of Preventive Medicine, Brigham and Women's Hospital and Harvard Medical School, Boston, MA 02115, USA

6 Centre for Clinical Epidemiology and Biostatistics, Hunter Medical Research Institute, University of Newcastle, Callaghan, NSW 2308, Australia

Cardiovascular disease (CVD) is the leading cause of death worldwide. Several CVD risk factors must be targeted to adequately address this multifactorial disease. Clinical trials have found that the consumption of dietary nitrate can acutely lower blood pressure, improve vascular health and reduce formation of blood clots. However, we do not yet understand the impact of long-term habitual dietary nitrate intakes and their relationship with CVD. We prospectively followed 62,535 women from the Nurses' Health Study who were free from diabetes, CVD and cancer at baseline in 1986. Information on diet and potential confounders was updated every 2 or 4 years with validated questionnaires. The main outcome was coronary heart disease (CHD), defined by the occurrence of non-fatal myocardial infarction or fatal CHD. Cox proportional hazard regression models were used to estimate relative risks (RR) and 95\% confidence intervals (CI). During 26 years of follow-up, 2257 cases of CHD were identified. The primary dietary source of nitrate intake was green leafy vegetables including lettuce and spinach. When comparing the highest quintile of nitrate intake with the lowest quintile, the RR was 0.77 (95\% CI; $0.67,0.87)$ after adjusting for age, race and baseline diagnosis of hypertension and hypercholesterolemia. After additional adjustment for the alternative healthy eating index excluding vegetable consumption, this inverse association was no longer significant [RR: 0.91 (95\% CI: 0.79, 1.04)]. Intakes of dietary nitrate may be inversely related to risk of CHD, however this association may not be independent of overall diet quality.

\subsection{The Effect of Vitamin D Supplementation in Patients with Heart Failure}

\section{L. Ge and B. R. Dong}

The Center of Gerontology and Geriatrics, West China Hospital, Sichuan University, No. 37 Guoxue Lane, Chengdu 610065, China 
Low 25-hydroxyvitamin D status has been associated with increased cardiovascular events in epidemiologic studies. We aimed to assess whether vitamin D supplementation improves cardiac function in adults with heart failure (HF) through an analysis of a meta-analysis of randomized controlled trials (RCTs). PubMed, Ovid and Medline were searched up to 1 June 2017 for randomized controlled trials that assessed the relationship between vitamin $\mathrm{D}$ supplementation and cardiac function (change in left ventricular ejection fraction (LVEF) (\%) and change in 6-min walk test (6 MW) (m) among adults with heart failure. Meta-analysis was conducted from six RCTs studies involving 482 participants (238 cases and 244 controls). Among six studies included in the meta-analysis, four reported the change in LVEF, four reported the $6 \mathrm{MW}$ outcomes. According to our meta-analysis, neither the LVEF (WMD $0.26 \%, 95 \%$ CI -0.25 to 0.78 ) nor the $6 \mathrm{MW}$ test (WMD $-0.04 \mathrm{~m}, 95 \% \mathrm{CI}-0.26$ to 0.18 ) were significantly affected by vitamin D supplementation in any of the subgroup comparisons. There is no evidence to support that vitamin D supplementation significantly benefits HF patients. However, given the relatively small number of enrolled patients, further studies are needed to elucidate this field.

2.5. Wholegrain and Dietary Fibre Intake on CVD, CHD, Stroke Incidence: Umbrella Analysis and Update of Effect Size Estimates

A. N. Reynolds ${ }^{1,2}$, G. Smith ${ }^{1}$, J. Mann ${ }^{1,2,3}$ and L. Te Morenga ${ }^{1,2}$

1 Department of Human Nutrition, University of Otago, Dunedin 9016, New Zealand

2 Edgar National Centre for Diabetes and Obesity Research, University of Otago, Dunedin 9016, New Zealand

3 Department of Medicine, University of Otago, Dunedin 9016, New Zealand

Numerous systematic reviews and meta-analyses exist on wholegrain or dietary fibre intake and incidence of cardiovascular disease (CVD), however quality varies. To consider the totality of evidence, an umbrella analysis was performed. An initial search to January 2017 identified nine systematic reviews reporting wholegrain or dietary fibre intake in the incidence of CVD, coronary heart disease (CHD), and stroke. The ROBIS tool for assessing risk of bias in systematic reviews was applied, and search updates to February 2017 identified the relevant pool of studies. This process identified 4.9 and 6.5 million person years of evidence on wholegrain and fibre intake in the incidence of CVD. The DerSimonian and Laird random effects model was used for high-versus-low intake comparisons, dose-response was considered with and without assuming linearity. Various sensitivity analyses were applied in the presence of heterogeneity $\left(\mathrm{I}^{2}>50 \%\right.$ or $\left.p<0.10\right)$. The strength of evidence was graded. Higher intakes of wholegrain, total fibre, and soluble fibre were consistently associated with decreased incidence of CVD, CHD. Total fibre showed the strongest relationship for CVD (RR0.76, 95\% CI 0.69-0.84), CHD (RR0.76, 95\% CI 0.70-0.83) and stroke (RR0.77, 95\% CI 0.680.87 ) in high-versus-low analysis. Vegetable fibre intake was associated with reduced incidence of $\mathrm{CHD}$ and stroke. No convincing associations were observed with cereal, fruit, and legume fibre. This umbrella analysis and update of effect size estimates produced results not dissimilar to the findings of the initial meta-analyses, however confidence in the effect size estimates and generalisability of results greatly improved.

\subsection{Cardiovascular Disease Risk Factors in Liver Transplant Recipients. Do They Impact Survival?}

L. Marsh ${ }^{1}$, K. McDowall ${ }^{2}$ and K. M. Dickinson ${ }^{1}$

1 Discipline of Nutrition and Dietetics, College of Nursing and Health Science, Flinders University, Adelaide, SA 5042, Australia

2 Department of Dietetics and Nutrition, Flinders Medical Centre, Bedford Park, SA 5042, Australia

The prevalence of cardiovascular disease risk factors among liver transplant candidates is increasing and there are few published studies on whether these risk factors impact on survival posttransplant. This study aimed to assess if the presence of pre-transplant cardiovascular disease risk factors, including obesity, hypertension, dyslipidaemia and hyperglycaemia, influence posttransplant mortality. A retrospective case note audit was conducted with the South Australian Liver Transplant Unit on adult patients with a minimum of five years follow-up. Patient pre-transplant 
cardiovascular disease risk factors were recorded from time of wait list assessment as well as height, actual body weight, blood pressure, blood lipids and blood glucose levels. Outcomes of interest were date and cause of death post-transplant. Of the 209 patients included in the study with risk factor data available, $52(25.1 \%)$ were obese, $60(28.8 \%)$ were hypertensive, $82(41.8 \%)$ were dyslipidaemic and $72(35.1 \%)$ were hyperglycaemic pre-transplant. Mean survival was estimated at 5769 (95\% CI 5244-6294) days (15.8 years) and survival rates were $92.8 \%$ at one year, $84.2 \%$ at five years and $65.5 \%$ at ten years. Overall, hyperglycaemia had a significant impact on post-transplant survival and was the most significant mortality predictor alone (HR: 1.99, 95\% CI 1.24-3.18, $p=0.004$ ) and in combination with obesity (HR: $2.34,95 \%$ CI 1.22-4.48, $p=0.01$ ). In summary, cardiovascular disease risk factors are common among patients pre-transplant and appear to influence survival. Assessment and management of risk factors such as hyperglycaemia is important and further research is recommended to determine how to minimise its associate mortality risk.

\subsection{No Effect of Consuming Eicosapentaenoic Acid Rich Fish Oil for 6 Months on Depression Severity in Patients with Heart Disease}

N. M. Berry ${ }^{1}$, A. M. Coates ${ }^{2}$, T. Air ${ }^{3}$, J. D. Buckley 2, J. Beltrame 4, S. Cai 2, L. Burres ${ }^{3}$,

P. R. C. Howe ${ }^{5}$ and G. Schrader ${ }^{3}$

1 University of East Anglia, Norwich NR4 7TJ, UK

2 University of South Australia, Adelaide, SA 5001, Australia

3 The University of Adelaide, Adelaide, SA, Australia

4 The Queen Elizabeth Hosptial, Adelaide, SA, Australia

5 University of Newcastle, Callaghan, NSW 2308, Australia

Omega-3 polyunsaturated fatty acids (n3-PUFA) have been shown to have positive effects on patients with mood disorders or cardiac diseases. Their effects on cardiac patients with depression remain uncertain. This randomised double-blind clinical trial evaluated the effects of n3-PUFA on depression severity in 73 participants (75\% male, $60.5 \pm 9.9$ years) with concurrent heart disease and depression who consumed four $1 \mathrm{~g}$ fish oil capsules/day containing $2.2 \mathrm{~g}$ eicosapentaenoic acid (EPA) and $0.2 \mathrm{~g}$ docosahexaenoic acid (DHA) for 6 months. Participants had angiographically-documented coronary artery disease and co-morbid depression (score of $\geq 16$ on the Centre for Epidemiologic Studies-Depression (CES-D) scale). Depression severity was also assessed using the clinician-rated Hamilton Depression Rating Scale (HAM-D). There was no difference at baseline in depression score (CES-D) between groups $(p=0.39)$. However, erythrocyte DHA levels at baseline were higher in the placebo group $(4.2 \pm 0.9 \%)$ than the treatment group $(3.8 \pm 0.7 \%)(p=0.04)$. The latter significantly increased their erythrocyte EPA + DHA levels relative to placebo $(2.7 \pm 1.8 \%$ vs. $-0.5 \pm 0.7 \%, p<0.001)$. Both treatment and placebo groups improved depression scores for CES-D $(\beta=-1.42, p=0.002)$ and HAM-D ( $\beta=-0.57, p=0.001)$ after controlling for gender and anti-depressant use but there was no time $x$ group interaction. There were no correlations between changes in erythrocyte EPA or DHA and changes in depression score. The data do not support an antidepressant benefit of EPA supplementation in depressed cardiac patients.

\subsection{Does Daily Consumption of Barley Affect Blood Cholesterol Concentrations? Systematic Review and Meta-Analysis}

Ramez Alhazzaa, Louise Harvey, Claire Larter and Dorothy Mackerras

Food Standards Australia New Zealand, Barton, ACT 2600, Australia

Soluble fibres such as $\beta$-glucan and arabinoxylan are present in barley (Hordeum vulgare) and thought to change blood cholesterol concentrations. Reductions in total and LDL cholesterol and increases in HDL cholesterol are beneficial health effects due to being inversely associated with coronary heart disease risk. A systematic review and meta-analysis were performed to assess how the consumption of barley, as wholegrain or minimally processed products, affects blood cholesterol concentrations. A broad search for barley, dietary fibres and blood cholesterol was conducted in PubMed, EMBASE and Cochrane CENTRAL in December 2014. In total, 295 articles were screened 
and seven strata ( $\mathrm{n}=185$ participants) from seven randomised controlled trials (RCTs) with low risk of bias were included in the review and the meta-analysis. Dietary intake of barley significantly changed total and LDL cholesterol concentration by -0.32 [95\% CI: $-0.42,-0.21]$ and -0.25 [95\% CI: $-0.32,-0.18] \mathrm{mmol} / \mathrm{L}$, respectively, without significantly affecting HDL cholesterol concentration. Daily barley dry weight achieving the effect ranged between $30 \mathrm{~g}$ and $175 \mathrm{~g}$, which cover the median daily cereal intake of $40 \mathrm{~g}$ in Australia. Dietary consumption of $\geq 30 \mathrm{~g}$ barley showed a consistent and significant reduction in blood total and LDL cholesterol concentrations, which can be explained by a plausible biological mechanism. However, the quality of the evidence for the relationship between barley intake and the reduction of blood total and LDL cholesterol concentrations has serious imprecision due to the low number of participants. Therefore, the quality of evidence has a moderate degree of certainty.

\subsection{Comparing the CVD Predictive Ability of Mediterranean Diet Adherence Scores: 10-Year Follow-Up of the ATTICA Study (2002-12)}

E. Georgousopoulou, D. Alyounis, N. D'Cunha, D. Mellor, N. Naumovski, C. Chrysohoou, C. Pitsavos and D. Panagiotakos

Australian National University, Australia

The Mediterranean diet (MD) is established as the most protective dietary pattern against Cardiovascular Disease (CVD). It is widely recommended as an efficacious lifestyle approach for CVD primary prevention. However, the tools assessing the level of adherence to MD have significant differences in methodology, and to date, there is no recognised gold standard. The present study aimed to compare some of the most widely cited scores (a-MED, t-MED, Italian Score and MedDietScore) for their performance in predicting CVD. The ATTICA study is a prospective study of individuals aged 18-89 years, living in the Athens metropolitan area, Greece, consisting of a random sample of 1514 males $(\mathrm{M})$ (mean age: $44.9 \pm 14.3$ years) and 1528 females (F) (45.6 \pm 13.5 years), recruited in 2001-2002 and followed-up for 10-years. CVD outcomes were diagnosed according to the ICD-10 criteria, with 2020 participants having valid information. Food intake was assessed with a validated Food Frequency Questionnaire. The 10-year CVD incidence was 15.7\% (M $=19.7 \%$ and $\mathrm{F}=11.7 \%, p<0.001)$. When scores were used separately in unadjusted models to predict 10-year CVD risk, only MedDietScore was inversely and independently associated with CVD risk (Hazard Ratio (HR) = 0.909, 95\% Confidence Interval (CI): 0.891, 0.928) and lowest -2logLikelihood $(-2 \log \mathrm{L}=166.416)$, which depicts superiority in prediction accuracy. The remaining scores were not independent CVD risk predictors, with lower predictive accuracy (a-MED: HR $=0.979,95 \%$ CI: 0.867, $1.11,-2 \log \mathrm{L}=487.169$; t-MED: $\mathrm{HR}=1.082,95 \%$ CI: 0.943, 1.24, $-2 \log \mathrm{L}=397.332$; Italian Score: $\mathrm{HR}=$ 1.01, 95\% CI: $0.881,1.16,-2 \log \mathrm{L}=487.123$ ). Results highlight the emerging need for a widely accepted tool for assessing adherence to MD patterns, which should be based on predicting ability and correct classification performance.

\subsection{NITRATE Improves Carbohydrate Metabolism by Increasing GLUT4 in Insulin-Sensitive Tissues in Obese Type 2 Diabetic Rats \\ Ghasemi Asghar ${ }^{1}$, Gheibi Sevda ${ }^{1,2}$ and Jeddi Sajad ${ }^{1}$ \\ 1 Endocrine Physiology Research Center, Research Institute for Endocrine Sciences, Tehran, Iran. \\ 2 Neurophysiology Research Center and Department of Physiology, Faculty of Medicine, Shahid Beheshti University of Medical Sciences. Tehran, Iran}

Decreased nitric oxide (NO) bioavailability contributes to the development of obesity and diabetes, and nitrate, a NO precursor, is a potential therapeutic agent for diabetes. This study aims to determine the effects of nitrate on carbohydrate metabolism and glucose-stimulated insulin secretion (GSIS) in type 2 diabetes. Male Wistar rats were divided into 4 groups: control, control+nitrate, diabetes, and diabetes + nitrate. Diabetes was induced using high-fat diet and a low dose of streptozotocin. Sodium nitrate $(100 \mathrm{mg} / \mathrm{L}$ in drinking water) was administered for two 
months. Serum levels of fasting glucose, insulin, and lipid profile were measured, and the insulin resistance/sensitivity indices were calculated every 2-weeks. At the end of the study, mRNA expression and protein levels of GLUT4 in soleus muscle and epididymal adipose tissue, and serum IL-1 $\beta$, were measured; additionally, glucose, pyruvate, and insulin tolerance tests were done. GSIS from isolated islets and islet insulin content was also determined. In diabetic rats, nitrate significantly improved carbohydrate metabolism and lipid profile but had no effect on GSIS and islet insulin content. Compared to untreated diabetic rats, nitrate administration restored GLUT4 mRNA expression and protein levels in soleus muscle (2.15-fold and $17 \%)$ and epididymal adipose tissue (3.44-fold and $22 \%$ ). Nitrate also restored elevated serum IL-1 $\beta$ in diabetic rats ( $4.7 \pm 0.5$ vs. $3.2 \pm 0.6$ $\mathrm{pg} / \mathrm{mL}, p=0.020$ ). In conclusion, long-term nitrate administration in type 2 diabetic rats improved carbohydrate metabolism by increasing mRNA expression and protein levels of GLUT4 in insulinsensitive tissues and decreasing inflammation.

\title{
2.11. Dose-Response Randomized Trial of Indian Curry Consumption on 24 hr CGM, Postprandial Insulinemia, and Triglyceridemia \\ S. Haldar ${ }^{1}$, S. C. Chia ${ }^{1}$, S. H. Lee ${ }^{2}$, J. Lim ${ }^{1}$, M. K. S. Leow ${ }^{1}$, E. C. Y. Chan ${ }^{2}$ and C. J. K. Henry ${ }^{1,3}$ \\ 1 CNRC, Singapore Institute for Clinical Sciences, Singapore \\ 2 Department of Pharmacy, NUS, Singapore, Singapore \\ 3 Department of Biochemistry, Yong Loo Lin School of Medicine, NUS, Singapore
}

Consumption of polyphenol rich foods have been associated with reduced risk of several chronic diseases including type 2 diabetes and cardiovascular diseases. Within the Asian dietary context, spices contribute appreciable amounts of dietary polyphenols. With the increasing popularity of Indian curries worldwide, we investigated the acute effects of two distinct doses of mixed spices (turmeric, cumin, coriander, Cayenne pepper, cinnamon, clove, and Indian gooseberry) and base vegetables (tomatoes, onions, ginger, and garlic), in quantities that are typically used in Indian curries when consumed with white rice, on $24 \mathrm{~h}$ glucose control using continuous glucose monitoring (CGM), on postprandial insulinemia and triglyceridemia (TG), and on urinary total polyphenol excretion (TPE) using Folin-Ciocalteu assay. The test meals and standardised meals during each intervention periods were isocaloric and matched for total vegetable content, as well as for macronutrient composition. Compared with Dose 0 Control (D0C), we found significant linear doseresponse reductions in postprandial incremental areas under the curve (iAUC) for CGM glucose during Dose 1 Curry (D1C) and Dose 2 Curry (D2C), respectively $(p<0.05)$. The postprandial iAUC of plasma insulin was also lower by approximately $15 \%$ during the D2C as compared with the D0C $(p=0.089)$. On the contrary, we found significant dose-dependent increases in postprandial TG with increasing doses of curry intake $(p<0.01)$. Significant increases in TPE with increasing curry doses across the three treatments were also noted. This study clearly demonstrates that consumption of mixed spices and base vegetables typically used in Indian curries has a significant modulatory role on postprandial metabolism.

\subsection{The Relationship between Dietary Patterns and Cognition in Older People with and without Type 2 Diabetes}

\author{
F. Zabetian_Targhi ${ }^{1}$, V. K. Srikanth ${ }^{1,2,3}$, K. J. Smith ${ }^{1}$, W. H. Oddy ${ }^{1}$, C. Moran ${ }^{2,3}$, W. Wang ${ }^{2}$ \\ and M. L. Callisaya ${ }^{1,3}$ \\ 1 Menzies Institute for Medical Research, University of Tasmania, Hobart, Tasmania, Australia \\ 2 Department of Medicine, Peninsula Health, Monash University, Melbourne, Victoria, Australia \\ 3 Department of Medicine, School of Clinical Sciences, Monash University, Melbourne, Victoria, Australia
}

Cognitive dysfunction is common in older people, particularly in those with type 2 diabetes (T2D). This study aimed to examine associations between diet and cognition and explore any differences in associations between those with and without T2D. A sample of 694 people $(n=350$ non-T2D, n =344 T2D) aged 55-90 years were recruited. The 80 items Cancer Council Food Frequency 
Questionnaire was used to assess dietary intake. Cognitive function was assessed using a battery of neuropsychological tests to create seven cognitive domain z-scores (verbal-memory, visual-memory, executive-function, language, processing-speed, visuospatial-functioning, and global-score). Three dietary patterns were derived from principal component analysis (traditional, prudent, and western). General linear models were used to assess the associations between dietary patterns and cognitive scores adjusting for age, sex, education, mood, and energy. The mean age was 70.0 years (SD 7.4) with $42.8 \%$ males. In adjusted models, a prudent diet was associated with better cognition in all domains $(p<0.05)$ except for executive-function $(p=0.70)$. A western diet was associated with poorer visual-memory $(p=0.03)$ and visuospatial-function $(p=0.004)$, and a traditional diet was associated with poorer visuospatial-function $(p=0.01)$. A significant interaction for T2D with diet suggested associations between a traditional diet and poorer global-cognition $(p=0.047)$, processing speed $(p=$ $0.03)$, and verbal memory $(p=0.03)$ was stronger in those without T2D. In conclusion, western and traditional diets may have negative effects on cognition in older people with some differences seen in people with and without T2D. Conversely, a prudent diet may have a critical role in maintaining cognitive function regardless of T2D diagnosis.

\subsection{InsuTAGTM: Relevance of Circulating Triglyceride Levels as a Predictor for Insulin Resistance}

R. N. Thota ${ }^{1}$, K. A. Abbott ${ }^{1}$, J. J. A. Ferguson ${ }^{1}$, M. Veysey ${ }^{2}$, M. Lucock ${ }^{3}$, S. Niblett ${ }^{2}$, K. King ${ }^{2}$ and M. L. Garg ${ }^{1}$

1 Nutraceuticals Research Program, School of Biomedical Sciences \& Pharmacy, University of Newcastle, Callaghan NSW 2308, Australia

2 School of Medicine \& Public Health, University of Newcastle, Callaghan NSW 2308, Australia

3 School of Environmental \& Life Sciences, University of Newcastle, Callaghan NSW 2308, Australia

Insulin resistance (IR) is a key etiological factor in type 2 diabetes, further contributing to the development of cardiovascular disease. The aim of this study was to investigate whether a novel physiologically relevant marker, InsuTAG ${ }^{\mathrm{TM}}$, a product of fasting insulin and triglyceride, can predict IR and metabolic syndrome (MetS). Data of 618 participants from the Retirement Health and Lifestyle Study were evaluated for the current study. IR was defined by homeostatic model assessment (HOMA-IR) scores $\geq 2.5$. Pearson correlations were used to examine the associations of HOMA-IR with InsuTAGTM and other markers. Predictions of IR from InsuTAG ${ }^{\mathrm{TM}}$ were evaluated using multiple regression models. Receiver operating characteristic (ROC) curves were constructed to measure the sensitivity and specificity of InsuTAG ${ }^{\mathrm{TM}}$ values in identifying IR and MetS. Youden's Index was used to determine the optimum cut-point for prediction of IR. InsuTAGTM was positively correlated with HOMA-IR ( $\mathrm{r}=0.86 ; p<0.0001)$. InsuTAG ${ }^{\mathrm{TM}}$ is a strong predictor of IR, accounting for $65 \%$ of the variation in HOMA-IR after adjusting for confounders. ROC curves showed that InsuTAG ${ }^{\mathrm{TM}}(0.93)$ was superior to other known lipid markers for predicting IR, with a sensitivity and specificity of $84.2 \%$ and $86.9 \%$. InsuTAG ${ }^{\mathrm{TM}} \geq 11.2$ was determined as the optimal cut-off for identifying IR. Prevalence of MetS was significantly higher $(p<0.0001)$ in subjects with InsuTAGTM values $\geq 11$.2. InsuTAG ${ }^{\mathrm{TM}}$ is correlated with metabolic disease risk factors and has high sensitivity and specificity for predicting IR in healthy older adults without diabetes. InsuTAG ${ }^{\mathrm{TM}}$ appears to be a feasible and reliable marker of IR and MetS.

\subsection{Diabetes Education: What Do Patients and Health Professionals Want?}

L. K. Andrew ${ }^{1}$, J. B. Keogh ${ }^{1}$, S. Dinmore ${ }^{2}$ and P. M. Clifton ${ }^{1}$

1 School of Pharmacy and Medical Sciences, University of South Australia, Adelaide, SA 5042, Australia

2 Teaching Innovation Unit, University of South Australia, Adelaide, SA 5042, Australia

Diabetes education plays an important role in self-management of type 2 diabetes, leading to improved blood glucose control and prevention of complications. The purpose of this study was (1) to explore the attitudes of patients and health professionals to an online education program and (2) determine its content by identifying patient needs and any confusion relating to type 2 diabetes selfmanagement. Qualitative semi-structured interviews were conducted with patients $(n=10)$ and 
health professionals $(n=7)$ and thematically analysed. Both patients and health professionals support an online program and patients prefer online compared to face-to-face delivery. Patients are confused about their medication and the difference between fasting blood glucose and $\mathrm{HbA1c}$. Two patients who knew and understood their HbA1c stressed the importance of the blood test in helping them manage long-term blood glucose control. Patients are both confused and frustrated by blood glucose fluctuations and dietary advice from health professionals to eat foods (e.g., fruit) that elevate blood glucose. Health professionals feel that type 2 diabetes education is essential for good selfmanagement and emphasised the important role of education linking good blood glucose control with preventing complications. Health professionals think that diet, particularly carbohydrates, portion size, and conflicting dietary advice, is most confusing for their patients. Dietary advice varies between health professionals who often provide their patients with one simple message regarding their diet. Based on these findings, the online diabetes program will include the following topics: basic diabetes information, diet, exercise, medication, complications, and the importance of blood glucose control.

\subsection{Long-Term Effect of Naked Oat on Type 2 Diabetes Mellitus (T2DM): One-Year Results of a RCT in China}

\section{Xu, J. Gu and Y. Li *}

Department of Nutrition and Food Hygiene, School of Public Health, Peking University, Beijing, China

* Corresponding Author

The present study aimed to assess the long-term effects of naked oat and structured nutrition therapy intervention on metabolic controls in Chinese adults with type 2 diabetes mellitus (T2DM). A two-staged, parallel-group, randomized, controlled trial was carried out in China. 445 participants aged 50-65 with T2DM were randomly assigned (1:1:1:1) to one of the four groups: usual care, diet, $50 \mathrm{~g}$ and $100 \mathrm{~g}$ naked oat, and diet group. During the first stage, participants were arranged to live in hotels and given a one-month centralized intervention. Then, participants returned home after 11 months of living in hotels. During the first stage, all three active groups showed significantly greater reductions of $\mathrm{HbA} 1 \mathrm{c}$ compared to the control group $(p<0.001)$. In addition, $100 \mathrm{~g}$ naked oat plus diet intervention produced a greater decrease of $\mathrm{HbA} 1 \mathrm{c}$ than diet intervention alone $(p=0.04)$. Decreases in $\mathrm{HbA} 1 \mathrm{c}$ persist in the three active groups after 12-month intervention $(p<0.001)$. The $100 \mathrm{~g}$ naked oat plus diet group reported the largest improvement of $\mathrm{HbA} 1 \mathrm{c}$ at month 12 , and mean differences in $\mathrm{HbA1c}$ change between $50 \mathrm{~g}$ or $100 \mathrm{~g}$ naked oat plus diet group and diet group were $-0.60 \%$ ( $p=$ $0.10)$ and $-0.74 \%(p=0.04)$. The long-term intervention of naked oat combined with structured nutrition therapy conferred greater benefits for the management of T2DM, rather than nutrition therapy intervention alone.

\section{in Type 2 Diabetes Patients \\ W. Zhang ${ }^{1}$, C. Liu ${ }^{1}$, L. Liu ${ }^{2}, \mathrm{H} . \mathrm{Yu}^{3}$, C. Liu ${ }^{2}$ and Y. An ${ }^{1}$ \\ 1 Feihe International Inc., Beijing 100016, China \\ 2 Chinese Nutrition Society, Beijing 100016, China \\ 3 Capital Medical University, Beijing 100016, China}

2.16. Consumption of a Milk-Based Formulation Decreased Fasting and Postprandial Plasma Glucose Levels

The demands for convenient nutrition solutions by the 130 million diabetes patients in China inspired us to develop a low-fat, milk powder-based formulation with ingredients that have been clinically shown to attenuate postprandial glycemic responses, including resistant starch, inulin, and L-arabinose. To investigate if chronic consumption of our formulation could improve glucose metabolism in type 2 diabetes patients, we recruited 110 subjects and conducted a double-blind, randomized, and placebo-controlled study. Subjects consumed our formulation or full-fat milk powder as control (30 g with breakfast and $15 \mathrm{~g}$ with dinner) daily for 90 days. Parameters such as blood glucose, insulin, and lipid profile were measured. Consumption of our formulation reduced 
fasting and 2-h postprandial glucose levels by $1.32 \mathrm{mmol} / \mathrm{L}(p<0.01)$ and $0.65 \mathrm{mmol} / \mathrm{L}(p<0.001)$, respectively. Conversely, consumption of full-fat milk powder had no effect on fasting or 2 -h postprandial glucose levels. We observed significant increase in $\mathrm{HbA1c}$ levels in subjects who consumed full-fat milk powder $(7.17 \%$ at baseline versus $7.67 \%$ after intervention, $p<0.05)$, possibly due to the Chinese New Year holidays that occurred during the intervention, in which large meals were usually consumed. By contrast, we did not observe such an increase in subjects who consumed our formulation. There was no change in insulin or blood lipid profiles in both groups. These results suggest that, as compared to full-fat milk powder, chronic consumption of our formulation may improve glycemic control in type 2 diabetes patients. Accordingly, our formulation is suitable to be part of nutrition solutions for diabetes patients.

\subsection{Variants of 57 Gene Associated with Diabetes in Chinese Population Born in and after Famine Period}

\section{A. Liu, C. Song and M. Wang}

National Institute for Nutrition and Health, Chinese Center for Disease Control and Prevention, Beijing 100016, China

Objective: Increasing studies demonstrated that genes and famine may interact in determining the risk of diabetes in adult life; however, the mechanism is not clear yet. The current study was conducted to explore whether people born in the 1960-1961 Chinese famine have the higher frequency of risk allele. Method: The sample for 1982 adults from cross-sectional 2010 China National Nutrition and Health Surveillance was utilized. The subjects born in 1960 and 1961 were selected as famine exposed group, and in 1963 were selected as unexposed group. The two groups were 1:1 matched by gender and birth district. Mass Array was used to detect the genotypes of 57 loci. Pearson square test or Fisher was used to analyze the difference of each genotype and allele of the two groups. Result: The frequency of risk allele of rs2028299, rs3923113, and 896854 in exposed group were 0.189, 0.128 , and 0.343 , respectively, and in unexposed group were $0.225,0.153$, and 0.310 , respectively. The frequency of risk allele is different between two groups: $\operatorname{rs} 2028299(=7.329, p=0.007), \operatorname{rs} 3923113$ $(=4.791, p=0.029)$, and $\mathrm{rs} 896854(=4.543, p=0.033)$. Subjects in exposed group had significant higher frequency of the rs896854T allele and less frequency of the rs2028299C allele and rs3923113C allele. There was no significant difference in the frequency of the rest 54 loci alleles between the two groups. Conclusions: Although 3 loci alleles were found to be different between the two groups, there is still no enough evidence to support that subjects born in famine have more chance to carry the diabetes risk gene.

\subsection{Dietary Pattern and Its Association with Cardio Metabolic Risks in Chinese Adults: The China Health and Nutrition Survey}

M. $\mathrm{Li}^{1}$ and $\mathrm{Z}$. Shi ${ }^{2}$

1 Division of Health Science, University of South Australia, Adelaide, SA 5042, Australia

2 Discipline of Medicine, University of Adelaide, Adelaide, SA 5005, Australia

(1) Background: Rapid social and economic change in China has brought about dramatic shift in diet and health among Chinese. We aimed to investigate the dietary pattern during 1991-2011 and its association with cardio metabolic risks in a longitudinal study among Chinese adults; (2) Methods: Adults participating in The China Health and Nutrition Survey were included. 3-day food consumption was collected by $24 \mathrm{~h}$ recall method. Anthropometric measures, blood pressure, fasting blood glucose, and blood lipids was collected in 2009. Dietary pattern was generated using principal components analysis. The association between dietary pattern and cardio metabolic risk was assessed with generalized linear regression adjusted for age, sex, and SES factors; (3) Results: "Traditional" pattern loaded with rice, meat, and vegetables, and "Modern" pattern had high loadings of fast food, milk, and deep fried food. "Traditional" pattern was inversely associated with cardio metabolic risks with linear slopes ranging from -0.15 (95\% CI: $-0.18,-0.12$ ) for hypertension to -0.67 (95\% CI: -0.73 , -0.60 ) for impaired glucose control. "Modern" pattern was associated positively with those factors 
with slopes ranging 0.10 (95\% CI: 0.04, 0.17) for high cholesterol to 0.42 (95\% CI: $0.35,0.49)$ for impaired glucose control; (4) Conclusion: Promoting healthy diet is urgent to tackle the uprising cardio metabolic disease epidemic in China.

\subsection{Genes Associated with Non-Th2 Asthma Are Differentially Expressed in the Airways of Obese Asthma}

\section{A. Thompson, B. S. Berthon, K. J. Baines, P. G. Gibson and L. G. Wood}

Centre for Healthy Lungs, Hunter Medical Research Institute, University of Newcastle, Callaghan, NSW 2308, Australia

The risk of developing asthma is increased by $50 \%$ in overweight and obese individuals; however, the causal links are still being elucidated. Inflammation is increased in both asthma and obesity, and may be a potential contributing factor. This observational, cross-sectional study aimed to investigate obesity driven inflammation in asthma by comparing sputum gene expression in obese $(\mathrm{n}=18)$ and non-obese $(\mathrm{n}=19)$ asthmatic adults. Lung function was measured by spirometry, and sputum was induced during hypertonic saline challenge. Microarray analysis and qPCR were used to identify genes that were differentially expressed between groups. Eighty genes were differentially expressed in obese versus non-obese asthmatics, with the majority being immune-related. Gene ontologies upregulated in obese asthma included the defense response, the inflammatory response, and the response to IFN- $\gamma$, which has been associated with non-T-helper 2 (Th2) asthma. In obese subjects, pro-inflammatory genes associated with IFN and TNF superfamilies (IFITM1, IL18RAP, and GBP1), neutrophil chemotaxis and migration (VNN2), triggering of the NLRP3 inflammasome (GBP5), and NF- $\mathrm{B}$ activation (IL17RB, IRF1) were upregulated. TFF3, a gene known to indirectly suppress inflammation, was downregulated in obese asthmatics. qPCR validated the microarray data confirming that IL18RAP ( $p=0.049)$ was upregulated and TFF3 $(p=0.009)$ was downregulated in obese asthmatics. This study identified several novel inflammatory genes associated with obesity and non-Th2 airway inflammation. These markers are potential therapeutic targets for this increasingly large and important patient population.

\subsection{The On/Off Diet: Effects of Week-On, Week-Off Energy Restriction Compared to Continuous Energy} Restriction-24 Month Data

\section{Headland 1,2, P. M. Clifton 1,2 and J. B. Keogh 1,2}

1 School of Pharmacy and Medical Science, University of South Australia, Adelaide, SA 5042, Australia

2 Sansom Institute for Health Research and Alliance for Research in Exercise, Nutrition and Activity (ARENA), University of South Australia, Adelaide 5001, Australia

Investigation of the effect of week-on, week-off energy restriction (IER), compared to continuous energy restriction (CER) on weight loss and weight loss maintenance after 12 and 24 months. Participants were overweight or obese (BMI $34.04 \pm 5.3 \mathrm{~kg} / \mathrm{m}^{2}, 51.8 \pm 11.3$ years) $(\mathrm{n}=107)$. Weight, body composition, and a fasting blood were taken at 0,8 weeks CER, and 16 weeks IER, and at 12 and 24 months. Attrition was $56 \%$ at 12 and $60 \%$ at 24 months. Weight loss was $-6.2 \pm 2.9 \mathrm{~kg}$ CER, $-6.4 \pm 2.3 \mathrm{~kg}$ IER; $(p=0.8)$ at 8 weeks and $-6.3 \pm 5.2 \mathrm{~kg}$ CER, $\mathrm{n}=27,-4.9 \pm 3.4 \mathrm{~kg}$ IER, $\mathrm{n}=20 ;(p=0.3)$ at 12 months. There was no difference between the groups for either fat mass or lean muscle mass after 8 weeks ER or 12 months ER. At 12 months, FGF-21 $(p<0.02)$ and triglycerides concentrations decreased $\left(-0.2 \pm 0.5 \mathrm{mmol} \mathrm{L}^{-1} p<0.01\right)$, while the decrease in fasting glucose approached significance $\left(-0.2 \pm 0.5 \mathrm{mmol} \mathrm{l}^{-1} p=0.06\right)$ with no difference between groups. Weight change between baseline and the 24 mnths between the groups approached significance $(-4.2 \pm 3.5 \mathrm{~kg}$ CER, $\mathrm{n}=24,-1.6 \pm 3.2 \mathrm{~kg}$ IER, $\mathrm{n}=19 ;(p=0.06))$. Weight increased between 12 and 24 months but was not significantly different (1.9 $\pm 2.5 \mathrm{~kg}$ CER, $3.1 \pm 3.7 \mathrm{~kg}$ IER $(p=0.2))$. Weight loss experienced in the first 12 months was the same in each group. However, by 24 months the difference in overall weight loss between the groups was approaching statistical significance, and regain experienced between 12 and 24 months was numerically larger in the IER. The large attrition, whilst being similar in both groups, limits generalisation of the results. This study is ongoing. 
2.21. Clinically Significant Weight Loss in the Upscaled PEACH ${ }^{\mathrm{TM}}$ QLD Community Program for Children above a Healthy Weight Range

C. J. Moores ${ }^{1}$, J. Miller ${ }^{1}$, L. A. Daniels ${ }^{2}$, H. Vidgen ${ }^{2}$ and A. M. Magarey ${ }^{1}$

1 Nutrition and Dietetics, College of Nursing and Health Sciences, Flinders University, Adelaide, South Australia, Australia

2 School of Exercise and Nutrition Sciences, Faculty of Health, Queensland University of Technology, Brisbane, Queensland, Australia

$\mathrm{PEACH}^{\mathrm{TM}}$-Parenting, Eating, and Activity for Child Hea-is a 6-month, parent-led weight management program for families with children above a healthy weight. The Program has previously demonstrated efficacy in RCTs and was recently upscaled in Queensland, Australia (2013-2016). This study aims to explore if clinically significant weight loss can be achieved in the upscaled PEACH ${ }^{\mathrm{TM}}$ QLD community-based child obesity program. PEACH ${ }^{\mathrm{TM}}$ QLD enrolled $n=926$ children $(n=817$ families) above a healthy weight range. Children were measured by trained facilitators at the beginning and end of the 6-month program. Clinically significant weight loss was defined as a reduction of $\geq 0.25 \mathrm{BMIz}$ units (US-CDC2000). Data are presented as mean [95\% CI: LL, UL]. Pre-post anthropometric data were available for $n=326$ children ( $60 \%$ female, mean age $8.7 \mathrm{y})$. At baseline, children were $22 \%$ overweight, $33 \%$ obese, and $45 \%$ morbidly obese (IOTF extended cut-points). At follow-up, children were $2 \%$ normal weight, $27 \%$ overweight, $34 \%$ obese, and $37 \%$ morbidly obese ( $P$ difference baseline to follow-up $<0.001$ ). Clinically significant weight loss was observed for $16 \%$ ( $n$ $=53)$ of children: mean decrease $-0.41[-0.52,-0.35]$. There were non-clinically significant BMIz score decreases for a further $61 \%(n=200)$, mean decrease $-0.10[-0.11,-0.09]$, and for the remaining $22 \%$ $(n=73)$, a mean increase of 0.08 [0.06, 0.11]. Clinically significant weight loss was observed in the upscaled PEACH ${ }^{\mathrm{TM}}$ QLD community health program. Further analyses are needed to determine demographics and lifestyle behaviour changes associated with clinically significant weight loss.

\subsection{Reliability of Compartmental Body Composition Measures in Adults Using GE iDXA: Implications for Nutrition Research}

A. D. Dordevic ${ }^{1}$, M. P. Bonham ${ }^{1}$, A. Ghasem-Zadeh ${ }^{2}$, A. Evans ${ }^{1}$, E. Barber ${ }^{1}$, K. Day 1,3, A. Kwok ${ }^{1}$ and $\mathrm{H}$. Truby ${ }^{1}$

1 Dept Nutrition, Dietetics \& Food, Monash University, Australia

2 Endocrinology Centre of Excellence, University of Melbourne, Australia

3 Dept Biomedical Sciences, University of Surrey, Australia

Nutrition intervention studies are increasingly using DXA to measure changes in body composition over time. The reliability and precision of any instrument is important in order to interpret whether measured changes in body compartments are significant and/or clinically relevant. This study aimed to measure the precision and reliability of body compartment measures obtained from the GE iDXA over a 3 month period. Weight stable adults $( \pm 2 \mathrm{~kg})$ aged $18-65$ years participated in total body composition scans, performed 3 times with at least 1 month between visits. Despite weight-stability, the coefficient of variation was high for each of the body compartment measures over 3 months (range 0.8-5.9\%). Precision-scans conducted on the same day with subject repositioning only resulted in $<2 \%$ variation. The coefficient of variation $(\mathrm{CV} \%)$ for visceral adipose tissue (VAT) was 31.6\%, significantly higher than other components. The least significant change for VAT mass over 3 months was $206.3 \mathrm{~g}$. VAT CV\% was negatively correlated with BMI $(-0.537, p<$ $0.001)$, total fat mass $(-0.489, p<0.001)$, and VAT mass $(-0.795, p<0.001)$, indicating that individuals with higher body fat and BMI $>25 \mathrm{~kg} / \mathrm{m}^{2}$ have a reduced variation in VAT measurement over 3 months. To accurately measure change in VAT via iDXA, it is recommended that participants require a BMI $>25 \mathrm{~kg} / \mathrm{m}^{2}$, or VAT mass $>500 \mathrm{~g}$. Any observed changes in VAT mass $<500$ grams should be interpreted with caution due to the lack of precision. 
2.23. Temporal Eating Patterns: Associations with Nutrient Intakes, Diet Quality, and Measures of Adiposity

R. M. Leech, A. Timperio, K. M. Livingstone, A. Worsley and S. A. McNaughton

Institute for Physical Activity and Nutrition (IPAN), School of Exercise and Nutrition Sciences, Deakin University, Geelong, Australia

Higher energy intake (EI) later in the day may be associated with poor diet quality and obesity. However, studies examining the distribution of eating occasions (EOs) across the day are rare. This study aimed to examine associations between temporal eating patterns, nutrient intakes, diet quality and measures of adiposity among Australian adults. Dietary data from two 24-h recalls collected during the 2011-12 National Nutrition and Physical Activity Survey were analyzed ( $\mathrm{n}=4544$ adults, $\geq 19$ years). Temporal eating patterns, based on the distribution of EOs across the day, were determined using latent class analysis. Diet quality was assessed using the 2013 Dietary Guidelines Index (DGI). Multivariate regression models assessed associations between temporal eating patterns, nutrient intakes, diet quality, and adiposity measures (weight status and central adiposity). Models were adjusted for potential confounders and energy misreporting. Three patterns, labelled "Conventional", "Later lunch", and "Grazing", were identified. Compared to a "Conventional" or "Later lunch" pattern, men and women with a "Grazing" pattern had lower DGI and higher intake of discretionary foods $(p<0.05)$. Among women, the "Grazing" pattern was associated with overweight/obesity ( $\mathrm{OR}=1.57,95 \% \mathrm{CI}$ : 1.15-2.13) and central adiposity ( $\mathrm{OR}=1.73,95 \% \mathrm{CI}: 1.19-2.50)$ after adjustment for potential confounders and energy misreporting. These associations were attenuated after excluding energy misreporters and adjusting for total EI. In conclusion, a "Grazing" temporal eating pattern was modestly but significantly associated with poorer diet quality and higher adiposity among women. Future research should consider the impact of energy misreporting on the relationship between temporal eating patterns and adiposity.

\subsection{Evaluation of Changes in Diet Quality during a Weight Loss Study in Obese Asthmatic Children}

S. R. Eslick ${ }^{1}$, M. E. Jensen ${ }^{1}$, C. E. Collins ${ }^{2}$ and L. G. Wood ${ }^{1}$

1 Centre for Healthy Lungs, Hunter Medical Research Institute, University of Newcastle, Callaghan, NSW, Australia

2 Priority Research Centre in Physical Activity and Nutrition, Faculty of Health and Medicine, University of Newcastle, NSW, Australia

The prevalence of obesity in asthmatic children is high and often associated with more severe asthma and poorer clinical outcomes. The objectives were (1) to examine changes in diet quality and clinical outcomes in obese asthmatic children following a weight loss intervention; and (2) to examine the association between diet quality, clinical outcomes, and weight loss success following a weight loss intervention. Twenty-eight obese asthmatic children, aged 8-17 years, completed a 10-week, dietinduced weight loss intervention involving dietary intake, anthropometry, lung function, asthma control, and clinical physical activity duration $(\mathrm{r}=-0.445, p=0.023)$ at baseline. This study demonstrated that acute weight loss can be safely achieved in obese asthmatic children using dietary intervention, resulting in significant improvements in asthma and diet quality.

\subsection{Polyphenol Levels Are Inversely Correlated with Body Weight and Obesity in an Elderly Population after 5 Years of Follow-Up}

Xiaohui Guo 1,2, Anna Tresserra-Rimbau ${ }^{1}$ and Rosa M. Lamuela-Raventòs 1,*

1 Department of Nutrition, Food Science and Gastronomy, XaRTA, INSA-UB, School of Pharmacy and Food Science, University of Barcelona, Barcelona, Spain

2 Department of Nutrition and Food Hygiene, School of Public Health, Peking University Health Science Center, Beijing, China

* Corresponding Author 
Few human studies have investigated the relationship between polyphenol intake and body weight. Our aim was to assess the relationship between urinary polyphenol levels and body weight. A cross-sectional study was performed with 573 participants from the PREDIMED (Prevenciòn con Dieta Mediterránea) trial (ISRCTN35739639). Total polyphenol levels were measured by a reliable biomarker, total urinary polyphenol excretion (TPE), determined by the Folin-Ciocalteu method in urine samples. Participants were categorized into five groups according to their TPE at the 5-th-year. Multiple linear regression models were used to assess the relationships between TPE and obesity parameters (body weight (BW), body mass index (BMI), waist circumference (WC), and waist-toheight ratio (WHtR)). After 5-year follow-up, significant inverse correlations were observed between TPE at the 5-th-year and BW ( $\beta=-1.004 ; 95 \% \mathrm{CI}-1.634$ to $-0.375, p=0.002)$, BMI $(\beta=-0.320 ; 95 \% \mathrm{CI}$ : -0.541 to $-0.098, p=0.005)$, WC $(\beta=-0.742 ; 95 \%$ CI: -1.326 to $-0.158, p=0.013)$, and WHtR $(\beta=-0.408$; $95 \%$ CI: -0.788 to $-0.028, p=0.036$ ) after adjustments for potential confounders. To conclude, a greater polyphenol intake may thus contribute to reducing body weight in elderly people at high cardiovascular risk.

\subsection{Pre-Intervention Brain Activation in Response to Visual Food Cues Predicts Weight Loss at Intervention}

M. O'Boyle, C. Kahathuduwa, T. Davis and M. Binks

Texas Tech University, TX 79409, USA

There is substantial variability in initial weight loss response to dietary restriction. Here we examine the ability of pre-intervention fMRI brain activation levels to predict change in weight $(\Delta \mathrm{Wgt})$ in a 3-week calorie restriction program. Data from 28 adults with obesity (BMI $30-39.9 \mathrm{~kg} / \mathrm{m}^{2}$ ) who participated in a randomized 3-week calorie restriction intervention (1120 kcal/day), compared to a diet derived from total meal replacement versus portion controlled typical foods, were examined. Body weight and brain activation (i.e., brain visual food-cue reactivity; fMRI-FCR) were measured. Pre-intervention fMRI-FCR data (i.e., contrasts of parameter estimates) were extracted from bilateral anterior cingulate, amygdala, dorsolateral prefrontal cortex, insula, nucleus accumbens, orbitofrontal cortex, and primary motor cortex. Linear correlations between $\Delta \mathrm{Wgt}$ and fMRI-FCR revealed that weight change was negatively correlated with pre-intervention activation of brain regions that exert inhibitory control over ingestion. These include the left anterior cingulate cortex $(\mathrm{r}=-0.447 ; p=0.01)$ and the left dorsolateral prefrontal cortex $(r=-0.536, p=0.003)$, as well as regions that regulate motor readiness to ingest, such as the left/right motor cortices $(r=-0.506, p=0.006$, and $r=-0.386, p=0.042)$. Our data suggest that lower levels of inhibitory control and increased motor readiness to ingest in response to food cue-measured pre-intervention is associated with attenuated weight loss. In the future, examination of pre-intervention brain activation levels may assist in identifying who is most likely to benefit from dietary calorie restriction.

\subsection{The Characteristics of Interventions That Effectively Prevent Excessive Gestational Weight Gain during Pregnancy: A Systematic Review and Meta-Analysis}

R. E. Walker 1, C. J. Bennett 1, M. L. Blumfield 1, S. M. Gwini 2, J. Ma 3, F. Wang 4, Y. Wan ${ }^{4}$ and H. Truby ${ }^{1}$

1 Department of Nutrition, Dietetics and Food, Monash University, Australia

2 Department of Epidemiology and Preventive Medicine, Monash University, Australia

3 Lanzhou University, Lanzhou, China

4 Zhejiang University, Hangzhou, China

Excessive maternal gestational weight gain (GWG) occurs in $50-70 \%$ of pregnancies and perpetuates generational obesity. Understanding why interventions work is critical to enabling effective translation. The rapid adoption of social media, and the use of novel medical delivery models, also requires exploration. This review aimed to describe the characteristics of international interventions aimed at preventing excessive GWG (diet, physical activity, lifestyle, eHealth, and 
medical) and to compare their efficacy via a systematic review of randomized controlled trials (RCTs) for preventing excessive GWG. Cochrane Library, CINAHL, EMBASE, LILACS, MEDLINE, PsycINFO, Scopus, and Chinese databases CNKI, Wanfang, and VIP were searched in April 2016 without date or language restrictions. Of 25425 screened references, 105 RCTs were identified. Metaanalyses (random-effects) of 75 trials estimated diet-only interventions as most effective in women without gestational diabetes mellitus (GDM) (WMD: $-2.60 \mathrm{~kg}$; $95 \% \mathrm{CI}:-3.69$ to $-1.60, p<0.001, I^{2}=$ 96.5\%). In women with GDM, interventions combining diet and activity were most effective (WMD: -2.76 ; $95 \%$ CI: -4.67 to $-0.85, p=0.005, I^{2}=97.7 \%$ ). The timing of commencement, intensity, frequency, duration, mode of delivery, and diet-type had no consistent effect on outcome. Interventions for preventing excessive GWG can be successful, with dietary interventions being most effective regardless of diet-type. Even if interventions commence later in pregnancy, are of low intensity and frequency, and are delivered to groups, they can still be effective. This suggests that low-cost, lowdose interventions can provide benefits and are responsive to local community needs and expectations, and culture is important in planning a successful strategy to prevent excessive GWG.

\subsection{Vitamin D and Its Metabolism Are Directly Associated with Improved Bone Quality in Elderly Patients \\ D. K. Sharma ${ }^{1}$, R. Stamenkov ${ }^{2}$, T. Robertson ${ }^{2}$, C. J. M. Stapledon ${ }^{2}$, G. J. Atkins ${ }^{2}$, L. B. Solomon ${ }^{2}$, P. M. Clifton ${ }^{1}$, P. H. Anderson ${ }^{1}$ and H. A. Morris ${ }^{1}$ \\ 1 School of Pharmacy and Medical Sciences, University of South Australia, Australia \\ 2 Centre for Orthopaedic and Trauma Research, University of Adelaide, Adelaide 5000, Australia}

Vitamin D supplementation with calcium markedly reduces the risk of fractures, although resulting increases in bone mineral density (BMD) are controversial. Such data suggest that this supplementation may not act through increasing BMD; rather, it may improve bone quality rather than quantity. The purpose of the present study was to investigate the mechanism by which vitamin $\mathrm{D}$ status affects bone quality in elderly hip fracture patients. Trabecular bone biopsies and serum samples were collected from hip fracture patients undergoing surgery for a hip replacement (70 females, 41 males) at Royal Adelaide Hospital. Radiographs were used to measure cortical thickness and medullary width. Trabecular bone structure was analysed by micro CT and serum $25(\mathrm{OH}) \mathrm{D}$, $1,25(\mathrm{OH})_{2} \mathrm{D}$, and parathyroid hormone $(\mathrm{PTH})$ by immunoassay. Serum $25(\mathrm{OH}) \mathrm{D}$ correlated positively with trabecular thickness $(\mathrm{r}=0.209, p<0.05)$ and negatively to the ratio of bone surface to bone volume $(\mathrm{BS} / \mathrm{BV})(\mathrm{r}=-0.214, p<0.05)$, both indicators of bone quality. In males, serum $25(\mathrm{OH}) \mathrm{D}$ correlated negatively with cortical medullary width, an indicator of bone resorption and accounted for $10 \%$ variability. No bone structural parameters were correlated with either PTH or $1,25(\mathrm{OH})_{2} \mathrm{D}$ levels. Furthermore, serum 25(OH)D and gender, together with bone mRNA levels for CYP27B1 (the enzyme that converts the prohormone $25(\mathrm{OH}) \mathrm{D}$ to $1,25(\mathrm{OH})_{2} \mathrm{D}$ ) and CYP24A1 (the enzyme responsible for degradation of vitamin D metabolites), account for $19 \%$ of the variability of $\mathrm{BS} / \mathrm{BV}(p$ $=0.001)$. These data indicate that serum $25(\mathrm{OH}) \mathrm{D}$ and its metabolism within bone to $1,25(\mathrm{OH})_{2} \mathrm{D}$ have direct effects on bone quality, thus emphasizing the need for maintaining adequate vitamin D status in the elderly to promote bone health.

\subsection{Japanese Young Females Are More Dissatisfied but at a Lower Risk of Disordered Eating Behaviours than Malaysian Chinese}

M. Kagawa, W. Gan, Y. Chin, M. Appukutty, J. Wong, B. Poh, Z. Mohd Shariff, M. Mohd Taib, S. Maruyama, T. Imai, and T. Takahashi

Curtin University, Perth 6102, Australia

Distorted body image may increase risks of unhealthy and often unnecessary weight-loss behaviours. While Japanese females are reported to be highly dissatisfied with their body, studies that compared severity of distorted body image with other ethnic groups are limited. The present study aimed to compare body dissatisfaction and its relationships with disordered eating behaviours, 
as well as self-esteem and depression risk between Japanese and Malaysian Chinese females. A total of 183 Japanese and 287 Malaysian Chinese females aged between 18 and 30 years completed questionnaires that included Body Satisfaction Scale (BSS), Figure Rating Scale (FRS), 26-item Eating Attitudes Test (EAT-26), Center for Epidemiologic Studies Depression scale (CES-D), and Rosenberg Self-Esteem Scale (RSES). While there were no significant differences in the body mass index (BMI) between the two ethnic groups, Japanese females expressed strong body dissatisfaction and reported thinner ideal physique compared with their Malaysian Chinese counterparts $(p<0.01)$. Japanese females also showed lower self-esteem than Malaysian Chinese; however, Malaysian Chinese showed higher risk of depression $(p<0.01)$. In addition, correlations between body dissatisfaction, self-esteem, and a risk of disordered eating behaviours were different between Japanese and Chinese Malaysian females. In conclusion, Japanese females have greater body dissatisfaction, while Malaysian Chinese have higher risks of developing disordered eating and depression. The results indicate the possibility of different aetiology and roles of body image and self-esteem in the development of disordered eating behaviours among different ethnicities.

2.30. Endogenously Synthesized n-3 PUFAs during Pregnancy Decreases Mammary Cancer Risk among the Offspring of Female Mice

\author{
J. M. $\mathrm{Li}^{1}{ }^{1}$ J. L. Gao ${ }^{1}$ and D. $\mathrm{Li}^{1,2}$ \\ 1 Department of Food Science and Nutrition, Zhejiang University, Zhejiang, China \\ 2 Institute of Nutrition and Health, Qingdao University, Qingdao, China
}

To investigate whether exposure to endogenously synthesized n-3 polyunsaturated fatty acids (PUFAs) during maternal pregnant period could decrease mammary cancer risk in later life of offspring and explore its potential mechanisms, wild-type C57BL/6J mice and transgenic fat-1 mice, which can synthesize n-3 PUFAs endogenously, were fed with a high-fat diet ( $43 \%$ energy) composed of n-6 PUFAs originating from safflower oil throughout pregnancy. Mammary tumorigenesis were induced by 7,12-dimethylbenz[a]anthracene (DMBA) in 7-week-old female offspring. Eight additional female offspring without DMBA treatment per group were used for mechanisms exploring. Offspring of fat-1 mice showed a significantly lower tumor incidence and longer latency to tumor appearance. The plasma concentration of $17 \beta$-estradiol, both in pregnant mothers on gestation day 19 and offspring aged 3 or 7 weeks, was significantly lower in fat-1 group. Offspring of fat 1 also exhibited delayed puberty onset, and their mammary glands contained enriched n-3 PUFAs in phospholipid fractions, decreased number of epithelial terminal end buds (TEBs, targets for malignant transformation), and increased differentiation of epithelial tree. Besides, reduced cell proliferation and increased apoptosis were observed in the TEBs structures of fat-1 offspring. Furthermore, differences in long noncoding RNA expression in mammary tissue were linked to DNA damage repair, cell proliferation, and differentiation. These findings suggest that prenatal exposure to endogenously synthesized n-3 PUFAs environment in utero has a protective effect on carcinogeninduced mammary tumors in later life by inducing epigenetic changes that modify susceptibility to breast cancer.

\title{
2.31. A 13 Month Pilot RCT to Assess Supplementation with LCn-3PUFA as an Adjunct Therapy for Non- Surgical Periodontal Treatment
}

A. M. Coates ${ }^{1}$, T. R. Fitzsimmons ${ }^{2}$, B. Chee ${ }^{2}$, B. Park ${ }^{2}$, S. Edwards' ${ }^{3}$, K. Kapellas ${ }^{4}$, P. R. C. Howe ${ }^{5}$ and P. M. Bartold ${ }^{2}$

1 Alliance for Research in Exercise, Nutrition and Activity (ARENA), Sansom Institute for Health Research, University of South Australia, Australia

2 Adelaide Dental School, University of Adelaide, Australia

3 Data, Design and Statistics Service, Adelaide Health Technology Assessment, Department of Public Health, University of Adelaide, Australia

4 Australian Research Centre for Population Oral Health, School of Dentistry, University of Adelaide, Australia 
5 Clinical Nutrition Research Centre, University of Newcastle, Australia

Periodontitis is characterized by chronic inflammation of gingival tissues. We evaluated the clinical efficacy of fish oil supplementation as an adjunct therapy for advanced chronic periodontitis in a 13 month randomized, controlled parallel trial. Participants $(10 \mathrm{M} / 23 \mathrm{~F}$, mean age $52 \pm 10$ years) consumed either fish oil (FO $\mathrm{n}=23 ; 1884 \mathrm{mg}$ long chain omega 3 polyunsaturated fatty acids (LCn3PUFA)/day) or placebo ( $\mathrm{n}=10 ; 2000 \mathrm{mg}$ soy oil/day) and were assessed at baseline and after 4, 7, 10, and 13 months for probing pocket depth (PPD) and clinical attachment (CAL). At baseline, 4 and 13 months fasting blood samples were assessed for LCn-3PUFA erythrocyte content. Good compliance to supplementation was evident with significant increases in erythrocyte LCn-3PUFA content over 13 months in the FO group compared with no change in the placebo group $(\Delta 3.2 \%$ vs. $-0.1 \%, p<0.001)$. Using a Poisson Generalized Estimating Equation model, there were significant reductions in mean PPD and CAL in both groups with group by time interactions for PPD, such that placebo had less PPD at 10 months compared with FO. There were also significant group by time interactions for CAL such that placebo had less CAL at 4 and 13 months compared with FO. The frequency of deep sites also declined significantly from baseline to 13 months in both groups (PPD > $=5 \mathrm{~mm}: 19$ to $4 \% \mathrm{FO}$ vs. 18 to $2 \%$ placebo $p<0.05$; CAL $\geq 6 \mathrm{~mm}: 18$ to $4 \% \mathrm{FO}$ vs. 12 to $0.5 \%$ placebo, $p<0.05)$, whilst periodontal treatment was effective in all participants; fish oil did not provide an added benefit.

Funding Source(s): Australian Dental Research Foundation grants (2013 and 2014); Novasel Australia LTD donated the capsules.

2.32. Metabolic Profile of People with Insulin Resistance, Pre-Diabetes, and Type 2 Diabetes: Implications for Nutrition Interventions

\section{K. A. Abbott, R. N. Thota and M. L. Garg}

Nutraceuticals Research Program, School of Biomedical Sciences \& Pharmacy, University of Newcastle, NSW, Australia

Nutrition intervention studies aimed at prevention of type 2 diabetes (T2D) often deliver conflicting results. Insulin resistance (IR) and pre-diabetes (PD) precedes development of T2D; however, prevention strategies do not distinguish between these states. The metabolic profiles of people with IR and PD may differ, potentially influencing outcomes in nutrition intervention studies. This study aimed to compare the metabolic profile of insulin-sensitive overweight/obese (ISO) and IR overweight/obese (IRO) individuals with those who have PD or T2D. Participants were recruited from the general public residing in the Greater Newcastle area, NSW, Australia, if they were overweight/obese or had been diagnosed with pre-diabetes or type 2 diabetes. HOMA-IR $\geq 2.5$ was used to categorize IR, and fasting blood glucose (FBG) was used to distinguish T2D $(\geq 7.0 \mathrm{mmol} / \mathrm{L})$ from PD (5.5-6.9 mmol/L). A total of 130 participants (age: $54.7 \pm 11.9$ yrs, BMI: $31.5 \pm 5.3$, males: $43.1 \%)$ were included in the analysis. Thirty participants had T2D (23.1\%); 48 (36.9\%) had PD. Of the remaining 52 participants, 20 had IR and 32 were IS. The BMI of ISO (28.5 \pm 3.7$)$ was significantly lower $(p<0.05)$ than IRO, PD, and T2D. No difference was seen between FBG of ISO vs. IRO individuals. Lipid profile was similar for IRO, PD, and T2D individuals. Overweight/obese individuals with IR have a similar metabolic profile to those with pre-diabetes and type 2 diabetes, suggesting hyperinsulinaemia is associated with metabolic dysfunction even in the absence of hyperglycaemia. This should be considered when tailoring nutrition interventions for people with insulin resistance versus those with pre-diabetes for improved health outcomes.

\subsection{Blood Mononuclear Cell Expression of GPR41 and GPR43 Is Associated with Plasma Scfa Levels in Asthma}

\section{R. McLoughlin, B. Berthon, K. Baines and L. Wood}

Priority Research Centre for Health Lungs, Hunter Medical Research Institute, University of Newcastle, Callaghan, NSW 2308, Australia 
Soluble fibres are fermented by bacteria in the colon to short chain fatty acids (SCFAs). Animal and human studies have suggested that anti-inflammatory effects of SCFAs occur via activation of $G$ protein coupled receptor's (GPRs) 41 and 43 . We have previously shown that in asthma, a single dose of soluble fibre improved lung function, reduced airway eosinophils, and upregulated airway gene expression of GPR41 and GPR43. In addition, 7 days supplementation with soluble fibre improved asthma control and reduced airway eosinophils. However the mechanisms of this remain unclear. This study investigated potential anti-inflammatory mechanisms of SCFAs by measuring GPR41 and 43 gene expression. We conducted a randomized, placebo-controlled 3-way crossover study $(\mathrm{n}=17$ asthmatics) of 7 days soluble fibre inulin ( $12 \mathrm{~g} /$ day), inulin+probiotic (multi-strain $>25$ billion CFU), and placebo supplementation. Peripheral blood mononuclear cells (PBMCs) were isolated using Ficoll density gradient. RNA was extracted from the PBMCs, and gene expression of GPR41/43 was measured by qPCR. Plasma SCFAs were measured by gas chromatography. Expression of GPR41/43 did not significantly change with any of the interventions; however, following inulin supplementation there were significant correlations between plasma SCFA butyrate levels and GPR41 ( $\left.r_{s}=0.7170, p=0.0026\right)$ and GPR43 $\left(r_{s}=0.6481, p=0.009\right)$ gene expression. Upregulation of GPR41/43 by SCFAs, in particular butyrate, may contribute to the anti-inflammatory response observed. We conclude that the mechanisms contributing to the anti-inflammatory role of inulin are likely to be mediated by SCFAs and involve a range of pathways that require further exploration.

\title{
2.34. Prospective Association of Dietary and Nutrient Patterns with Mortality: Findings from a 20-Year Follow-Up Study in China
}

\author{
Y. Melaku ${ }^{1}$, T. Gill ${ }^{1}$, A. Taylor ${ }^{1}$, R. Adams ${ }^{2}$ and Z. Shi ${ }^{2}$ \\ 1 Adelaide Medical School, The University of Adelaide, Australia \\ 2 Health Observatory, Discipline of Medicine, The University of Adelaide, Adelaide, SA, Australia
}

Studies on food/nutrient patterns associated with mortality risk are scarce. Using data from the Chinese Health and Nutrition Survey (CHNS), we determined the prospective association of dietary and nutrient patterns with mortality among 15,990 adults aged 18 years and over. Dietary intake data were collected using a 24-h recall method for three consecutive days, for each individual across nine waves (1989-2011). Dietary and nutrient patterns were identified in seven of the nine waves (19912009) using factor analysis. Factor scores (pattern scores) were calculated. We used overall mean scores of the patterns as an exposure. Mortality was recorded at each wave. To assess the associations between the pattern scores and all-cause mortality, hazard ratios (HRs) were determined using Cox proportional hazard regression. Two dietary (traditional and modern) and two nutrient (plant- and animal-sourced) patterns were identified. Over the median 8.9 years of follow-up, 1243 incident deaths were recorded. After adjusting for potential confounders, participants in the third tertile (highest intake) of the modern dietary pattern had a 58\% decrease (HR $=0.42 ; 95 \%$ CI: $0.33-0.53$ ) in mortality risk, compared to those in the first tertile (lowest intake). Participants in the third tertiles of the animal- and plant-sourced nutrient patterns had a $29 \%$ decrease ( $\mathrm{HR}=0.71 ; 95 \% \mathrm{CI}$ : $0.58-0.87)$ and a $107 \%$ increase ( $\mathrm{HR}=2.07 ; 95 \% \mathrm{CI}: 1.71-2.51)$ in mortality risk, respectively, compared to those in the first tertiles. Greater adherence to a modern dietary and/or an animal-sourced nutrient pattern is associated with a lower risk of all-cause mortality in China.

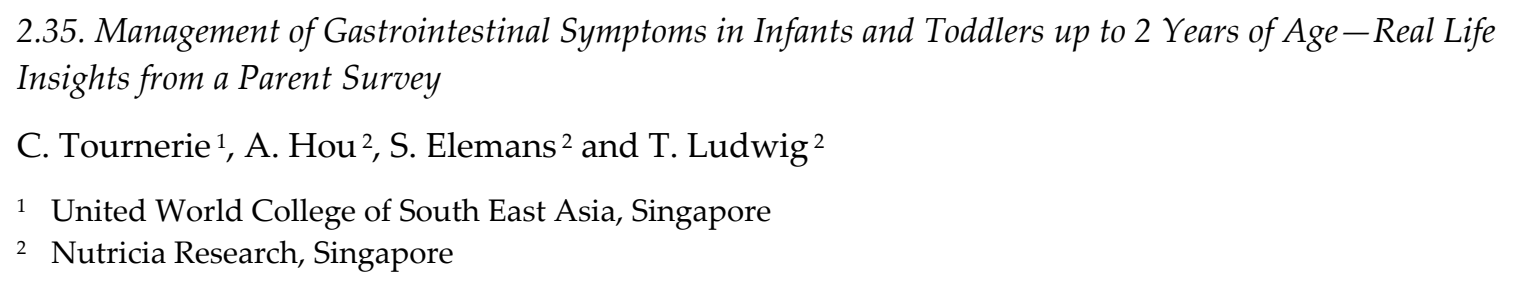

The 'real life' behavior of parents in relation to functional gastrointestinal disorders (i.e., infant colic, constipation, and regurgitation) and related signs and symptoms is not well researched. Methods: An anonymous electronic survey was distributed in April 2017 to employees of a nutrition 
company in two locations (Singapore and The Netherlands). Parents that had in the past five years children with gastrointestinal complaints in the first two years after birth were eligible to participate. Results: Of the 77 respondents, $68.8 \%$ were European, $24.7 \%$ Asian, and 6.5\% of other nationalities. Participants consulted a doctor (68.8\%), the internet (63.6\%), family members (50.6\%), fellow mothers $(36.4 \%)$, friends $(31.2 \%)$, books or magazines $(20.8 \%)$, or social media $(15.6 \%)$. According to the participants, the most useful information sources were medical doctors $(27.3 \%)$, the internet $(22.1 \%)$, and the family (15.6\%). In brief, a broad spectrum of a multitude of remedies was reported, ranging from physical manipulation to usage of medication and over the counter remedies. For example, up to 10 remedies were reported by one respondent, in which $44 \%$ of respondents reported usage of three or more different kinds of remedies. The most obvious difference between locations was the usage of formula thickeners, which was reported by $21.6 \%$ of the respondents in the Netherlands and none in Singapore. Conclusion: This survey indicates the common usage of a very high diversity of remedies for the alleviation of early life gastrointestinal issues with frequent usage of multiple remedies in one infant.

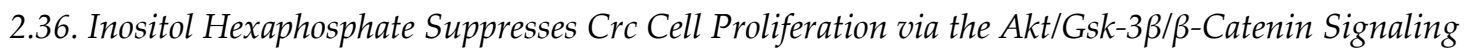 Cascade in DMH-Induced Rats}

\section{W. Yu, C. Liu, X. Li, F. Yang, L. Cheng, C. Liu and Y. Song}

1 School of Public Health, Qingdao University Medical College, China

2 School of Nursing, Qingdao University Medical College, China

3 School of Basic Medicine, Qingdao University Medical College, China

Colorectal cancer (CRC) is common worldwide, and most treatments for CRC have undesirable side effects. Many researchers have demonstrated that inositol hexaphosphate (IP6) has potent anticarcinogenic activity against CRC and no apparent toxicity to normal cells. However, the underlying mechanism is still unclear. In this study, we investigated the anticancer and antiproliferative properties of IP6 in CRC and its possible mechanisms during this chemopreventive process. We examined the expression of genes related to the PI3K/Akt and Wnt pathways at the transcriptional and translational levels in a DMH-induced rat CRC model following IP6 administration. In addition, we also conducted cell proliferation analysis. The results demonstrated that IP6 could inhibit tumors, in terms of tumor incidence, number, weight, and volume in DMHinduced rats. Additionally, Akt and C-myc mRNA levels were significantly decreased. IP6 was also shown to downregulate Akt, pAkt, pGSK-3 $\beta$, and C-myc protein expression and upregulate $\mathrm{p} \beta$ catenin protein expression. Furthermore, tumor tissues from IP6-treated rats showed decreased proliferation. In conclusion, the anti-proliferative effect of IP6 may be related to crosstalk between the PI3K/Akt and Wnt pathways, revealing a potential mechanism of CRC inhibition by IP6 in our model.

\subsection{Propolis Beneficially Modulated Gut Micriobiota and Ameliorated Dextran Sulfate Sodium-Induced Colitis in Rats}

K. Wang ${ }^{1,2,3}$, X. Jin ${ }^{4}$, M. You ${ }^{2}$, R.K. Le Leu ${ }^{5}$, D.L. Topping ${ }^{3}$, M.A. Conlon ${ }^{3}$, L. $\mathrm{Wu}^{1}$ and F. $\mathrm{Hu}^{2}$

1 Institute of Apiculture Research, Chinese Academy of Agricultural Sciences, Beijing, China

2 College of Animal Sciences, Zhejiang University, Hangzhou, China

3 CSIRO Health and Biosecurity, Adelaide, Australia

4 College of Food Science and Nutritional Engineering, China Agricultural University, Beijing, China

5 Central and Northern Adelaide Renal and Transplantation Service, Royal Adelaide Hospital, Adelaide, Australia

Background: Propolis is an important hive product and is considered beneficial to health. However, evidence of its potential for improving gut health is still lacking. Here, we use rats to examine whether dietary supplementation with propolis could be used as a therapy for ulcerative colitis. Methods: Rats were fed with a Western style diet alone (controls) or supplemented with different amounts of Chinese propolis $(0.1 \%, 0.2 \%$, and $0.3 \%)$ to examine effects on acute colitis 
induced by 3\% dextran sulfate sodium (DSS) in drinking water. Results: Propolis at $0.3 \%$, but not lower levels, significantly improved colitis symptoms compared with the control group, with a lesspronounced disease activity index (DAI) $(p<0.001)$, a significant increase in colon length/weight ratio $(p<0.05)$, and an improved distal colon tissue structure as assessed by histology. Although short chain fatty acid levels in digesta were not altered by propolis supplementation; the use of $16 \mathrm{~s}$ rRNA phylogenetic sequencing revealed a significant increase in gut microbial diversity after $21 \mathrm{~d}$ of $0.3 \%$ propolis supplementation compared with controls. Propolis induced a significant increase in bacteria belonging to the Proteobacteria and Acidobacteria phyla. Conclusion: This is the first study to demonstrate that propolis can attenuate DSS-induced colitis and provide new insight into dietmicrobiota interactions during inflammatory bowel disease.

\subsection{Quantification of Short Chain Fatty Acids in Fermented Food and Beverages}

\section{P. A. Gill ${ }^{1}$, J. G. Muir ${ }^{1}$, M. C. van Zelm ${ }^{2}$ and P. R. Gibson ${ }^{1}$}

1 Department of Gastroenterology, Central Clinical School, Monash University, Melbourne, VIC, Australia

2 Department of Immunology and Pathology, Central Clinical School, Monash University and Alfred Hospital, Melbourne, VIC, Australia

Short chain fatty acids (SCFAs) are produced within the colon as the result of colonic fermentation of 'dietary fibre'. SCFA promote gut health and also have systemic effects. Increased delivery of SCFA to the body may be desirable. SCFAs are also present in many fermented foods and beverages, representing a poorly characterised oral source of SCFA. We aimed to quantify the SCFA levels present within fermented products to determine their potential for therapeutic use. Commonly consumed and readily available fermented foods and beverages were chosen for analysis. Representative samples of food and beverages were collected and pooled. SCFA were quantified using gas chromatography with a flame ionization detector (GC-FID). Heptanoic acid was used as an internal standard. Dairy products were extracted using diethyl ether with a valeric acid internal standard. Samples were run in triplicate with variation $<5 \%$. Sample data was expressed as $\mathrm{mg} / \mathrm{g}$, as well as mg per serving size. Acetate was the most abundant SCFA detected and was highest in pepper sauce $(124 \mathrm{mg} / \mathrm{g})$ and apple cider vinegar $(67 \mathrm{mg} / \mathrm{g})$. Blue vein cheese $(5.5 \mathrm{mg} / \mathrm{g})$ and Gherkin $(0.6$ $\mathrm{mg} / \mathrm{g}$ ) contained the highest levels of butyrate and propionate, respectively. After adjusting for serving sizes, kombucha (1200 mg) and apple cider vinegar $(1000 \mathrm{mg})$ provide the most SCFA, mainly as acetate. Oral sources of SCFA via the consumption of fermented foods may provide another approach for elevating circulating levels of SCFA. The potential immune-modulating and epigenetic effects require further investigation.

\subsection{Ten Weeks of Increased Protein Consumption Raises Plasma TMAO Concentrations in Healthy Older Males: A 10 Week Randomised Controlled Trial}

S. M. Mitchell ${ }^{1}$, C. J. Mitchell ${ }^{1}$, A. M. Milan ${ }^{1}$, N. Zeng ${ }^{1}$, F. Ramzan ${ }^{1}$, P. Sharma ${ }^{1}$, E. A. Rettedal ${ }^{2}$, S. Knowles $^{2}$, N. Roy ${ }^{2}$, S. J. Orena ${ }^{3}$, S. H. Zeisel ${ }^{3}$, A. Sjödin ${ }^{4}$, K.-H. Wagner ${ }^{5}$ and D. Cameron-Smith ${ }^{1}$

1 Liggins Institute, New Zealand

2 AgResearch, New Zealand

3 University of North Carolina, NC, USA

4 Copenhagen University, Denmark

5 University of Vienna, Austria

High protein diets (HPD) are enriched in choline and L-carnitine. These are precursors in bacterial metabolism, generating trimethylamine- $N$-oxide (TMAO). TMAO is positively associated with increased risk for cardiovascular disease events. However, HPDs are increasingly recommended to maintain physical function in the elderly. In this study twenty-nine healthy men (74.2 \pm 3.6 years) were randomised to consume a controlled HPD $(1.6 \mathrm{~g} / \mathrm{kg} / \mathrm{day})$ or APD $(0.8 \mathrm{~g} / \mathrm{kg} / \mathrm{day})$ for 10 weeks. Faecal and fasting blood samples were collected at baseline and in the final week. Quantification of plasma TMAO, creatinine, choline, and betaine was performed using liquid chromatography-stable isotope dilution-multiple reaction monitoring mass spectrometry. The faecal 
microbiota is being characterised by $16 \mathrm{~S}$ rDNA gene sequencing using the Ion Torrent platform, and preliminary compositional results are available. Plasma concentration of TMAO increased only in the HPD group $(8.3 \pm 4.7 \mathrm{M}$ to $29.1 \pm 31.5 \mathrm{M}, p=0.008)$. Creatinine decreased in the APD group $(86.5 \pm$ $20.4 \mathrm{M}$ to $78.2 \pm 16.6 \mathrm{M}, p=0.002)$. Choline and betaine were unchanged. Gene sequencing of variable regions 1-9 has thus far shown no significant differences in faecal microbial alpha-diversity (Chao1) within or between groups. HPD can substantially increase circulatory TMAO in healthy older men, and this should be considered when making recommendations for optimal protein intake for the elderly. Further analysis of faecal microbial composition may establish correlations between TMAO production and gut microbial community structure.

\subsection{Defining Dairy Intolerance: Symptoms of Dairy Intolerance in the Absence of Lactose Malabsorption}

A. M. Milan ${ }^{1}$, A. Shrestha ${ }^{1}$, H. J. Karlström ${ }^{2}$, J. A. Martinsson ${ }^{2}$, N. J. Nilsson², M. P. G. Barnett ${ }^{3,4}$ and D. Cameron-Smith ${ }^{1}$

1 Liggins Institute, University of Auckland, New Zealand

2 Linköping University, Linköping, Sweden

3 Food Nutrition \& Health Team, AgResearch Limited, New Zealand

4 High-Value Nutrition National Science Challenge, New Zealand

Lactose intolerance (LI) research often reports a subset of individuals referred for intolerance testing that are not diagnosed as lactose malabsorbers, despite self-reported digestive symptoms following dairy consumption. Yet, the characteristics of non-lactose dairy intolerance (NLDI) have not been reported. The current study aimed to identify individuals with self-reported dairy intolerance, excluding those clinically identified as lactose malabsorbers, and characterise the diary intolerant digestive response. Healthy young women were assessed for lactose intolerance by standardised lactose challenge and diagnosed based on blood glucose, hydrogen breath test, and subjective symptoms. Tolerant controls $(n=10)$, lactose intolerant malabsorbers $(n=10)$, and NLDI subjects ( $n=20$; self-report intolerant but not lactose intolerant) consumed $750 \mathrm{~mL}$ of milk to examine dairy digestive responses. Self-reported symptoms were recorded by visual analog scale half-hourly over $3 \mathrm{~h}$. Tolerant subjects experienced no notable increase in symptoms. NLDI subjects reported increased abdominal distension, rumbling, and bloating following milk consumption, which subsided by 90 to $120 \mathrm{~min}$. In contrast, LI subjects experienced these symptoms throughout the postmeal period, without relief. However, NLDI subjects experienced belching throughout the post-meal period, unlike LI subjects. Further, LI subjects experienced delayed onset (past $90 \mathrm{~min}$ ) of abdominal cramps and fecal urgency, which were not experienced in NLDI subjects. We propose that NLDI can be defined by a distinct subset of digestive symptoms experienced after dairy consumption, independent of lactose tolerance. Rather than lower gastrointestinal symptoms associated with lactose malabsorption, NDLI is characterised by early onset upper gastrointestinal symptoms that resolve rapidly.

\subsection{Resistant Starch Prevents High-AGE, Diet-Induced CKD Progression by Remodeling the Gut Microbiome and Suppressing Complement}

M. Snelson, S. M. Tan, V. Thallas-Bonke, T. V. Nguyen, G. C. Higgins, R. Lindblom, K. C. Sourris, M. Ziemann, A. El-Osta, M. E. Cooper and M. T. Coughlan

Department of Diabetes, Central Clinical School, Monash University, Melbourne, Australia

Highly processed foods contain Advanced Glycation End-products (AGEs), which have been linked to the development of diabetes complications. Diet-derived AGEs largely escape digestion and are fermented by gut bacteria; however, it is not known whether AGEs can alter gut microbial ecology, leading to the progression of chronic kidney disease (CKD). This study aimed to investigate if diet-derived AGEs could worsen CKD progression, and whether CKD progression could be arrested via remodeling the gut microbiome using resistant starch (RS) supplementation. These studies utilized a mouse model of type 2 diabetes, the $\mathrm{db} / \mathrm{db}$ mouse, in which a low- or high-AGE 
diet, plus or minus $25 \%$ RS, was supplied for 10 weeks. Gut microbiota composition was assessed using 16S-rRNA sequencing of cecal digesta. Transcriptomic profiling of renal cortex was determined by RNA-Sequencing. In db/db mice, consumption of a high AGE diet led to worsening of renal injury (albuminuria), enhanced intestinal permeability (determined by in vivo FITC-labelled dextran clearance), and a shift in the Firmicutes to Bacteriodetes ratio compared to low AGE-fed db/db mice. RS restored Firmicutes:Bacteriodetes and significantly reduced albuminuria. Gene set enrichment analysis showed an upregulation in the complement cascade in high AGE-fed $\mathrm{db} / \mathrm{db}$ mice, which was normalized by RS. These studies indicate that: (i) Dietary AGEs can worsen CKD via gut microbiome remodeling and complement activation and (ii) Diet therapy with RS can improve renal disease progression and dampen innate immune system activation. RS may be a readily applicable therapy for protecting the kidney in individuals at risk of CKD.

\subsection{Understanding Growth: Pacific Islands Families Study, Growth Patterns 2 to 14 Years, and Association with Metabolic Markers}

E. Rush, V. Obolonkin, L. Plank, M. Smith, L. Iuistini, F. Savila and E. Tautolo

University of Auckland, Auckland 1010, New Zealand

From the year 2000, the longitudinal Pacific Islands Families (PIF) Study has followed the growth and development of more than 1000 Pacific children born at Middlemore Hospital, South Auckland. We aimed to identify at age 14 years weight trajectories from 2 to 14 years and assess their association with metabolic risk factors. A nested subsample (100 girls, 104 boys) was randomly selected from the cohort for indepth measures including a blood sample to measure metabolic risk. Growth patterns were determined by standardizing ages of measurement at 2, 4, 6, 9, 11, and 14 years, ranking the weight of the children on age, and estimating linear and quadratic regressions on age. Quintiles by slope and separately by intercept for each child allowed determinations of trends of growth trajectory, 2 to 14 years of age, with metabolic measures at 14 years; birthweight; and physiological, nutrition, lifestyle, and geographical variables measured previously. The steeper the weight trajectory from 2 to 14 years, the higher the concentrations of insulin; C-peptide; leptin; and markers of liver function, kidney function, and inflammation (all $p<0.01$ ). Children with increased weight at age 2 years (intercept) showed a similar trend for increasing concentrations of these metabolic markers. A relatively rapid growth pattern or a higher body weight in early childhood was associated with higher risk for metabolic disease. Monitoring growth trajectories may help target interventions to optimize growth, nutrition, and physical activity.

\subsection{Sleep Duration and Overweight/Obesity in Preschool-Aged Children: A Prospective Study of Up to 48,922 Children}

Y. Wan ${ }^{1}$, H. Liu ${ }^{2}$, F. Wang ${ }^{1}$ and D. Li ${ }^{1}$

1 Department of Food Science and Nutrition, Zhejiang University, Hangzhou, China

2 Jiaxing Maternity and Child Health Care Hospital, Jiaxing, China

High prevalence of obesity is conventionally attributed to increased intake of energy-dense food and reduced physical activity. However, in recent years, sleep duration has been proposed as a possible modifiable factor of obesity. To examine the association between sleep duration and overweight/obesity in preschool-aged children, a total of 48,922 3-year old children were included in the present study as baseline and were followed up to 5 years of age. Prevalence ratios and risk ratios were used to assess the association between sleep duration and risk of overweight/obesity. In baseline cross-sectional analyses, the adjusted prevalence ratios (95\% confidence interval) of overweight (with 11-12 $\mathrm{h}$ of sleep being considered the reference group) for children sleeping $\leq 10 \mathrm{~h}$ and $\geq 13 \mathrm{~h}$ were $1.13(1.06-1.20)$ and $1.16(1.09-1.24)$, respectively, whereas the adjusted prevalence ratios $(95 \%$ confidence interval) of obesity were $1.25(1.11-1.40)$ and 1.25 (1.11-1.42). In longitudinal analyses, the adjusted risk ratios (95\% confidence interval) of overweight for children sleeping $\leq 10 \mathrm{~h}$ and $\geq 13 \mathrm{~h}$ were 1.48 (1.26-1.74) and $1.13(0.96-1.34)$, while adjusted risk ratios (95\% confidence interval) of 
obesity were $1.77(1.30-2.40)$ and 1.19 (0.85-1.66). Restricted cubic splines regression supported Ushaped curvilinear associations between sleep duration and overweight/obesity in both crosssectional and longitudinal analyses. In conclusion, both short and overlong sleep duration are associated with a higher risk of overweight/obesity in preschool-aged children. Optimizing sleep duration may be an important, modifiable intervention for overweight and obesity.

\subsection{Evaluating the Effect of Habitual Dietary Fat Consumption on Fat Taste Sensitivity in Twins: A Randomized Controlled Trial}

A. Costanzo ${ }^{1}$, C. Nowson ${ }^{1}$, L. Orellana ${ }^{1}$, K. Duesing ${ }^{2}$ and R. Keast ${ }^{1}$

1 Deakin University, Geelong, VIC, Australia

2 CSIRO Health \& Biosecurity, North Ryde, NSW, Australia

Individuals with impaired oral fatty acid sensitivity (fat taste) have reduced satiety responses after consuming fatty foods, leading to passive overconsumption. Habitual consumption of dietary fat may modulate sensitivity to fat taste, with high consumption decreasing sensitivity (increasing fat taste thresholds (FTT)) and low consumption increasing sensitivity (decreasing FTT). No study has assessed this while controlling for weight change. The objective was to assess the effect of low-fat or high-fat dietary intake on FTT, whilst maintaining weight. A randomised, controlled trial including 44 twin pairs (mean age 43.7 (15.4 SD) years, 34 monozygotic, 10 dizygotic, 33 female, 10 male, and 1 gender-discordant) was conducted, with each twin within a pair randomly allocated to a low-fat ( $<20 \%$ energy fat) or high-fat ( $>35 \%$ energy fat) diet over 8 weeks. FTT was assessed by a 3-alternate forced choice methodology and transformed to an ordinal scale (FT rank), and dietary intake using six 24-h food records. Linear mixed regression models were fitted, accounting for twin pairs as a random factor. Compliance to dietary intervention was maintained throughout the trial (low-fat: $17.0 \%$ (6.6) energy fat; high-fat: $39.0 \%$ (6.8) energy fat). Weight did not change in either group after 8 weeks (low-fat: $-1.5 \mathrm{~kg}, 95 \%$ CI: [-5.5, 2.4], $p>0.05$; high-fat: $+0.5 \mathrm{~kg},[-3.5,4.4], p>0.05)$. FT rank changed in both groups after 8 weeks (low-fat: -4.2 , [-5.6, -2.9], $p<0.001$; high-fat: +1.5, [0.2, 2.8], $p<$ 0.05 ; low-fat vs. high-fat: $-5.8,[-4.7,-6.9], p<0.001)$. This study provides evidence that habitual fat consumption modulates fat taste sensitivity. Understanding this mechanism may aid in reducing passive overconsumption of fatty foods.

\subsection{Anthocyanins Derived from Sweet Cherries Reduce Obesity Markers in High Fat-Fed Mice}

\section{L. Blackhall ${ }^{1}$, R. Berry ${ }^{1}$, D. P. Geraghty ${ }^{2}$, K. D. K. Ahuja ${ }^{2}$ and J. T. Walls ${ }^{1}$ \\ 1 School of Medicine, University of Tasmania, Australia \\ 2 School of Health Science, University of Tasmania, Australia}

Obesity is a major risk factor for cardiovascular disease, type 2 diabetes, and some cancers, making it a global health concern. The drugs used to treat obesity elicit a myriad of side effects, making non-drug treatments desirable. There is growing evidence that the water-soluble flavonoids, anthocyanins, have a positive impact on many diseases, but only limited research on their potential anti-obesity actions has been conducted. The aim of our study was to determine the efficacy of sweet cherry anthocyanins as a treatment and preventative therapy for obesity in mice. Six week prevention (PT) and 10-week reversal trials (RT) were conducted in parallel. Ninety-six male mice were fed a high fat diet (HFD, 36\% wt/wt fat) and were evenly split into PT or RT. Mice in the PT were randomised into either treatment (Tx) or control (Cx) with PT-Tx mice receiving $40 \mathrm{mg} / \mathrm{kg}$ body weight anthocyanin. In the RT, after 6 weeks HFD, the mice were split into Tx and Cx, with Tx mice receiving anthocyanin for the final 4 weeks of the trial. Mice in the PT gained 19\% less weight $(p<$ 0.05 ) following 6 weeks supplementation with anthocyanin. No significant difference was found in the 10 week RT; however, the trend observed suggested reversal of obesity might be possible with a longer treatment phase. Mice that were fed a HFD that included anthocyanin gained significantly less weight than mice that did not receive the anthocyanin. This suggests that cherry anthocyanin might reduce weight gain in those consuming a HFD. 
2.46. Interventions Designed to Reduce Gestational Weight Gain Can Reduce the Incidence of GDM: Systematic Review and Meta-Analysis

C. J. Bennett 1, R. E. Walker ${ }^{1}$, M. L. Blumfield ', S. M. Gwini ${ }^{2}$, J. Ma 3, F. Wang ${ }^{4}$, Y. Wan ${ }^{4}$, H. Dickinson ${ }^{5}$ and H. Truby ${ }^{1}$

1 Department of Nutrition, Dietetics and Food, Monash University, Australia

2 Department of Epidemiology and Preventive Medicine, Monash University, Australia

3 Lanzhou University, Lanzhou, China

4 Zhejiang University, Hangzhou, China

5 Department Obstetrics and Gynaecology, Monash, Australia

Excessive gestational weight gain (GWG) increases the risk of gestational diabetes mellitus (GDM). Many interventions have reduced GWG. However, the effect on GDM is still unknown. This systematic review (SLR) aimed to (i) evaluate the impact of interventions designed to prevent excessive GWG on the incidence of GDM, and (ii) examine if effects differ by geographical region and body mass index (BMI). An SLR of randomised controlled trials (RCTs) was conducted without date limits using seven international databases and three Chinese databases. RCTs that reported a primary/secondary aim to reduce excessive GWG and the incidence of GDM were considered. Two authors independently identified and assessed the included studies. Meta-analysis data are reported for GDM incidence with interventions covering diet, physical activity (PA), and lifestyle (diet plus PA). Of 20,517 manuscripts screened, 37 were included for analysis $(n=12,942)$. Diet interventions reduced the risk of GDM by $44 \%$ (RR: $0.56,95 \%$ CI: $0.36-0.87$ ), while PA interventions reduced the risk by $38 \%$ (RR: $0.62,95 \%$ CI: 0.50-0.78). Both lifestyle interventions and BMI did not significantly alter the risk. PA interventions from Southern Europe reduced GDM risk by $37 \%$ (RR: $0.63,95 \%$ CI: $0.50,0.80$ ). Both diet and lifestyle interventions conducted in Asia resulted in a 62\% (RR: $0.38,95 \%$ CI: $0.24,0.59$ ) and $32 \%$ (RR: $0.68,95 \%$ CI: $0.54,0.86$ ) reduction in GDM, respectively. Interventions designed to prevent excessive GWG can reduce the risk of GDM. However, due to physiological and/or behavioural responses to interventions, the 'one size fits all' approach is not supported.

\author{
2.47. The Impact of Eating a Meal, Snack, or Not Eating during a Simulated Nightshift on Sleepiness, \\ Hunger, and Gastric Complaints \\ C. Gupta 1, J. Dorrian"1, S. Centofanti 1, A. Coates 1, D. Kennaway 2, G. Wittert 2, L. Heilbronn 2, \\ P. Catcheside ${ }^{3}$, M. Noakes ${ }^{4}$, J. Stepien ${ }^{1}$, D. Chandrakumar ${ }^{1}$, D. Coro ${ }^{1}$ and S. Banks ${ }^{1}$ \\ 1 University of South Australia, Australia \\ 2 Adelaide University, Australia \\ 3 Flinders University, Australia \\ 4 CSIRO, Australia
}

Shiftworkers often report eating during a nightshift. It is unknown what effect the size of the meal eaten at night has on hunger and gastric complaints, as shiftworkers are eating when the body is primed for sleep. This study explored the impact of eating a meal, a snack, or not eating on sleepiness, hunger, and gastric complaints. 22 healthy non-shiftworking males $(n=13)$ and females $(n=9)$ aged $18-39$ years (age $\mathrm{M} \pm$ SD: $24.2 \pm 5.2$ years, BMI $\left.23.8 \pm 2.1 \mathrm{~kg} / \mathrm{m}^{2}\right)$ underwent four simulated nightshifts. Participants were randomly allocated to a condition: meal ( $n=7$; males:females, 4:3), snack $(n=7 ; 4: 3)$, or no eating $(n=8 ; 5: 3)$. At $0030 \mathrm{~h}$, participants consumed either a meal comprising $30 \%$ of $24 \mathrm{~h}$ energy intake (meal condition), a snack comprising $10 \%$ of $24 \mathrm{~h}$ energy intake (snack condition), or did not eat during the nightshift (no eating condition). Total $24 \mathrm{~h}$ energy intake and macronutrient content was kept constant across conditions. At 2100 h, 2330 h, 0230 h, and 0500 h, participants completed sleepiness, hunger, and gastric complaint scales. Mixed model analyses revealed that, at $0500 \mathrm{~h}$, participants in the meal condition were significantly sleepier $(p<0.05)$, felt more full $(p<0.01)$, were less hungry $(p=0.001)$, and tended to report more stomach upset $(p=0.10)$ and bloating $(p=0.28)$ compared to the snack condition, with no significant differences between the snack and no eating conditions. Eating a large meal during the nightshift leads to increased sleepiness 
and gastric complaints. To minimise sleepiness and gastric complaints during the nightshift, shiftworkers should consider the size of the meal eaten.

\subsection{Post-Exercise Ingestion of Carbohydrate, Protein, and Water: A Meta-Analysis for Effects on Subsequent Athletic Performance}

D. McCartney, B. Desbrow and C. Irwin

School of Allied Health Sciences and Menzies Health Institute Queensland, Griffith University, Gold Coast, Australia

Endurance athletes often undertake consecutive training sessions with limited recovery time between exercise bouts (e.g., $\leq 4 \mathrm{~h}$ ). Nutritional strategies that optimise post-exercise recovery in these situations are therefore important. This two-part review investigated the effect of consuming carbohydrate $(\mathrm{CHO})$ and protein (PRO) with water following exercise on subsequent endurance exercise performance. Studies that measured endurance exercise performance ( $\geq 5$ min duration $) \leq 4 \mathrm{~h}$ after a standardised exercise bout (any type) under the following control vs. intervention conditions were included: Part-1: Water (W) vs. CHO ingested with an equal volume of water (CHO-W); and, Part-2: $\mathrm{CHO}-\mathrm{W}$ vs. protein ingested with $\mathrm{CHO}$ and an equal volume of water (PRO-CHO-W), in which $\mathrm{CHO}$ or energy intake was matched. Studies were excluded if extraneous dietary/pharmacological constituents were administered. Random-effects meta-analyses and metaregression analyses were conducted to evaluate intervention efficacy. Part-1: 44 trials $(n=487)$ were reviewed. Ingesting $-\mathrm{W}\left(106 \pm 51 \mathrm{~g} \mathrm{CHO} ; 0.82 \pm 0.52 \mathrm{~g} \mathrm{CHO} \mathrm{kg}^{-1} \cdot \mathrm{h}^{-1}\right)$ improved exercise performance compared to W $(1.6 \pm 0.6 \mathrm{~L}) ; \% \Delta$ Mean Power Output $(\mathrm{MPO})=4.18,95 \%$ CI: 3.37, $4.99\left(\mathrm{I}^{2}=42.7\right)$. Improvement was attenuated when participants were "fed" ( $\leq 4 \mathrm{~h}$ post-prandial) as opposed to "fasted" ( $>10 \mathrm{~h}$ post-prandial) on commencing the initial exercise bout $\left(p=0.029 ; \mathrm{R}^{2}=0.30\right)$. Part-2: 14 trials $(\mathrm{n}=135)$ were reviewed. Ingesting PRO-CHO-W $\left(33.6 \pm 25.5 \mathrm{~g}\right.$ PRO; $0.48 \pm 0.35 \mathrm{~g}$ PRO $\left.\cdot \mathrm{kg}^{-1}\right)$ did not affect exercise performance compared to CHO-W $\left(112 \pm 59 \mathrm{~g} \mathrm{CHO} ; 0.60 \pm 0.24 \mathrm{~g} \mathrm{CHO} \mathrm{kg} \mathrm{BM}^{-1} \cdot \mathrm{h}^{-1}\right.$; $1.4 \pm 0.6 \mathrm{~L}) ; \% \Delta \mathrm{MPO}=0.55,95 \% \mathrm{CI}:-0.52,1.62\left(\mathrm{I}^{2}=72.9\right)$. Athletes with limited time for nutritional recovery between consecutive training sessions should prioritise $\mathrm{CHO}$ and fluid ingestion to enhance subsequent endurance exercise performance.

\subsection{Consuming Low Doses of Alcohol with a Sugar-Containing or Artificially-Sweetened Mixer on Simulated Driving Performance}

\section{B. Brickley, B. Desbrow, D. McCartney and C. Irwin}

Menzies Health Institute Queensland, School of Allied Health Sciences, Griffith University, Gold Coast, Australia

The Australian National Drug and Alcohol Research Centre (NDARC) recommends specific drinking guidelines to ensure breath alcohol concentrations (BrAC) remain $\leq 0.05 \%$. However, these recommendations may be inappropriate for individuals consuming alcohol without carbohydrate, which results in higher BrACs. This study investigated effects of ingesting alcohol (as per NDARC guidelines) with and without carbohydrate on BrACs and simulated driving performance. Thirtytwo participants (16 male; $22 \pm 6 \mathrm{y} ; 71 \pm 11 \mathrm{~kg}$ ) completed two trials in a randomised single-blinded study. Participants performed a baseline drive (Drive 1), and then an experimental drive (Drive 2) following alcohol consumption (males: $20 \mathrm{~g}$; females: $10 \mathrm{~g}$ ). Alcoholic beverages contained either 25 g sucrose or aspartame (AS), matched for sweetness. Primary measures of driving performance were lateral control (standard deviation of lane position, i.e., lane swerving, and number of lane departures, i.e., lane crossings) and risk taking (total number of overtaking manoeuvres and maximum speed whilst overtaking). BrAC and subjective ratings (mood, arousal, intoxication, impairment, and willingness to drive) were also assessed. BrAC at the onset of Drive 2 was significantly reduced in the sucrose trial $(0.021 \pm 0.009 \%$ vs. $0.030 \pm 0.011 \%)$. Two males provided a breath sample $>0.05 \% \mathrm{BrAC}$ under the AS treatment, whereas two females had no detectable BrAC under the sucrose treatment. Neither beverage significantly influenced measures of simulated 
driving (Drive 1 vs. 2). Subjective ratings did not differ significantly between treatments. Ingesting alcohol in quantities advised by the NDARC results in no detectable impairment in simulated driving. However, the likelihood of exceeding the legal driving $\mathrm{BrAC}$ is increased when alcohol is consumed with non-nutritive mixers.

\subsection{The Effect of Cooking on the Nitrate, Nitrite, and Nitrosamines Content for Use with a FFQ: A Systematic Review}

N. F. McMahon ${ }^{1}$, T. G. Pavey ${ }^{2}$, B. Desbrow ${ }^{3}$ and M. D. Leveritt ${ }^{1}$

1 School of Human Movement and Nutrition Sciences, University of Queensland, St. Lucia, QLD, Australia

2 School of Exercise \& Nutrition Sciences, Queensland University of Technology, Kelvin Grove, QLD, Australia

3 School of Allied Health Sciences, Griffith University, Gold Coast, QLD, Australia

Background/Aims: Dietary nitrate $\left(\mathrm{NO}_{3}^{-}\right)$improves vascular function and enhances exercise performance when consumed adequately. Vegetables offer the highest source of $\mathrm{NO}_{3}{ }^{-}$(per $100 \mathrm{~g}$ ). Considering cooked vegetable intake is higher than fresh sources, the objective of this systematic review was to determine the impact of cooking processes (such as boiling and blanching) on $\mathrm{NO}_{3}{ }^{-}$ and nitrite $\left(\mathrm{NO}_{2}{ }^{-}\right)$concentration values in food and beverages. Methods: Studies were included if extractable $\mathrm{NO}_{3}{ }^{-}$and $\mathrm{NO}_{2}{ }^{-}$data was available for foods or beverages, and provided a comparison between fresh and cooked samples. Results: Of the 2682 vegetable samples tested, only $10 \%$ of nitrate from vegetable sources and $18 \%$ of nitrite from vegetable sources were cooked and analysed for their $\mathrm{NO}_{3}{ }^{-}$and $\mathrm{NO}_{2}{ }^{-}$content. The weighted mean $\mathrm{NO}_{3}{ }^{-}$concentrations losses from fresh vegetable samples due to the cooking processes were boiling $(53.99 \%)>$ steamed $(51.35 \%)>$ blanched $(47.39 \%)>$ fried $(45.5 \%)>$ microwaved $(26.52 \%)>$ baked $(2.25 \%)$. Conclusions: The lack of data provides an interesting avenue for future research investigating the quantification of dietary intakes of $\mathrm{NO}_{3}^{-}, \mathrm{NO}_{2}^{-}$, and nitrosamines, particularly when cooked vegetable intake is higher than raw. From the analytical data it is clear the effect of thermal processing or treatment should be taken into account to when assessing the levels of $\mathrm{NO}_{3}{ }^{-}$that may be present in vegetables.

\subsection{Does a Preference for Salty or Sweet Foods Impact on Overall Energy Intake or Weight Status?}

\section{G. A. Hendrie, H. J. Lease, M. A. Rebuli and D. N. Cox}

\section{CSIRO Health and Biosecurity, Adelaide SA 5000, Australia}

As "taste" is a primary driver of food choice, it would be useful to understand how the sensory properties of diets influence total dietary energy intake (EI) and weight status. A database of 720 frequently consumed foods, described by a trained sensory panel for basic tastes (sweetness and saltiness) and fatty-mouthfeel, was systematically applied to all foods consumed in the 2011-2013 Australian National Nutrition and Physical Activity Survey. Data from the face-to-face $24 \mathrm{hr}$ dietary recalls from children and adults $(n=12,153)$ were analysed, and total nutrient and sensory properties of diets described. To understand the contribution of food groups to total nutrient and sensory intake, all foods and beverages were assigned to food groups consistent with the Australian Guide to Healthy Eating. There were positive significant correlations between the nutrient and sensory properties of reported diets. In adults, discretionary foods contribute $44 \%$ to total sugar intake compared to $35 \%$ of total sweetness; $32 \%$ to total sodium intake and $25 \%$ to total saltiness; and $33 \%$ to total fat intake and $23 \%$ to total fatty-mouthfeel. Normal weight adults derived a greater contribution of sweetness from core foods than overweight/obese adults. Regression models for adults, controlling for age, sex, and BMI, revealed that fatty-mouthfeel $(\beta=0.492)$, saltiness $(\beta=0.223)$, and sweetness $(\beta=0.189)$ were significant predictors of EI and explained $56 \%$ total variance $(p<0.01)$. Sensory properties of diet were poor predictors of weight status. Key findings suggest core foods can provide sensory stimulation, and that fatty-mouthfeel drives EI more than saltiness or sweetness. 
2.52. Cruciferous and Total Vegetable Intakes Are Inversely Associated with Subclinical Atherosclerosis in Older Adult Women

B. P. Bondonno 1, L. C. Blekkenhorst 2, J. R. Lewis ${ }^{2}$, R. J. Woodman ${ }^{3}$, A. Devine 1, W. H. Lim ${ }^{4}$, K. Zhu ${ }^{5}$, L. J. Beilin ${ }^{1}$, P. L. Thompson 5 , R. L. Prince ${ }^{2}$ and J. M. Hodgson ${ }^{1}$

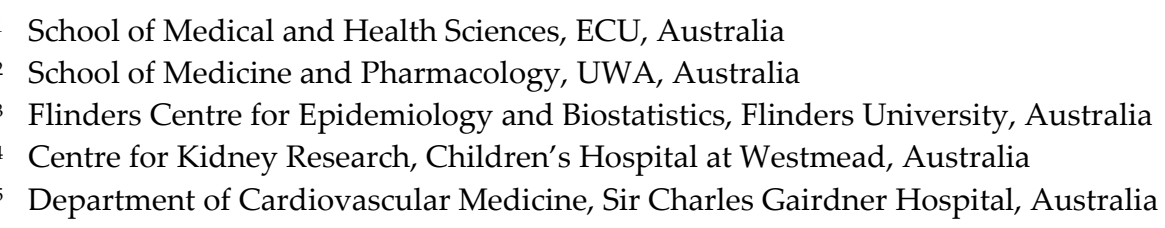

Dietary patterns rich in vegetables are inversely associated with markers of atherosclerosis such as common carotid artery intima-media thickness (CCA-IMT) and plaque. Few studies have explored associations of vegetable intake alone and whether different types of vegetables, containing different phytochemical profiles, are important. We investigated associations of total vegetable intake and specific vegetables grouped according to phytochemical constituents with CCA-IMT and carotid plaque severity in a cohort of women aged $\geq 70$ years. Intakes of total vegetables and vegetable types (cruciferous, allium, yellow/orange/red, leafy greens, and legumes) were calculated at baseline (1998) using a food frequency questionnaire. In 2001, CCA-IMT $(n=954)$ and focal carotid plaques $(n=968)$ were assessed using high-resolution B-mode carotid ultrasonography. Baseline mean (SD) of total vegetable intake was 199.9 (78.0) $\mathrm{g} / \mathrm{d} ; 2.7$ (1.0) serves/d. Women consuming $\geq 3$ serves of vegetables each day had approximately 4.6-5.0\% lower mean CCA-IMT $(p=0.014)$ and maximum CCA-IMT ( $p$ $=0.004)$ compared to participants consuming $<2$ serves of vegetables. For each $10 \mathrm{~g} / \mathrm{d}$ higher cruciferous vegetable intake, there was an associated $0.006 \mathrm{~mm}(0.8 \%)$, lower mean CCA-IMT $(p<$ $0.01)$, and $0.007 \mathrm{~mm}(0.8 \%)$ lower maximum CCA-IMT $(p<0.01)$. Other vegetable types were not associated with CCA-IMT. No associations were observed between vegetables and plaque severity. Increasing vegetable intake with a focus on cruciferous vegetables may have benefits for the prevention of subclinical atherosclerosis in older adult women.

\subsection{The Acute Effect of Magnesium Supplementation on Endothelial Function: A Pilot Study}

\section{J. Murphy ${ }^{1,2}$, J. B. Keogh ${ }^{1}$ and P. M. Clifton ${ }^{1}$}

1 School of Pharmacy and Medical Sciences, Alliance for Research in Exercise, Nutrition and Activity (ARENA), Sansom Institute for Health Research, University of South Australia, Australia

2 Dublin Institute of Technology and Trinity College Dublin, Ireland

Magnesium $(\mathrm{Mg})$ deficiency may be an important factor in the process of endothelial dysfunction, an early event in the development of atherosclerosis. Our aim was to determine the acute effect of an oral $\mathrm{Mg}$ supplement compared to no supplement on flow-mediated-dilatation (FMD) as a measure of endothelial function. Nineteen participants (mean age 39 years, BMI 22.9 $\mathrm{kg} / \mathrm{m}^{2}$ ) completed this randomised cross-over study. Blood pressure and FMD measurements were recorded, and blood samples were taken before participants drank $200 \mathrm{~mL}$ water with or without an over the counter $\mathrm{Mg}$ supplement (450 $\mathrm{mg}$ and $300 \mathrm{mg}$ for men and women respectively) with repeat measurements at 60 and $120 \mathrm{~min}$. There was no time by treatment interaction for absolute change in FMD $(p=0.267), \operatorname{SBP}(p=0.182), \operatorname{DBP}(p=0.383)$, or MAP $(p=0.201)$. Mg increased $(p=0.037$ compared with control) from $0.86 \pm 0.06 \mathrm{mmol}$ at baseline and $0.88 \pm 0.05 \mathrm{mmol}$ at $60 \mathrm{~min}$ to $0.91 \pm 0.06 \mathrm{mmol}$ at $120 \mathrm{~min}$ post-supplementation ( $p<0.001 \mathrm{from}$ baseline and $p=0.002$ from $60 \mathrm{~min}$ ). An acute dose of $\mathrm{Mg}$ as an oral supplement had no effect on absolute change in FMD, SBP, DBP, or MAP over time, compared with no supplement control. Serum $\mathrm{Mg}$ significantly increased following supplementation. There appears to be no benefit of $\mathrm{Mg}$ supplements on FMD or blood pressure.

\subsection{Anthocyanin Cyanidin-3-Rutinoside Promotes Vasodilation and Protects Methylglyoxal-Induced Vascular Dysfunctions in Rat Aorta}

T. Thilavech ${ }^{1}, \mathrm{M}$. Abeywardena ${ }^{2}$ and S. Adisakwattana ${ }^{1}$ 
1 Department of Nutrition and Dietetics, Faculty of Allied Health Sciences, Chulalongkorn University, Bangkok 10330, Thailand

2 CSIRO Health \& Biosecurity, Kintore Avenue, Adelaide, SA 5000, Australia

Cyanidin-3-rutinoside (C3R), a natural anthocyanin present in high levels in certain fruits and vegetables, has been reported to possess anti-oxidant, hypoglycemic, and anti-glycation properties. Methylglyoxal (MG) is a highly reactive dicarbonyl aldehyde by-product of glycolysis. MG is significantly elevated in the hyperglycemic state and serves as a major precursor of advanced glycation end products that underpins the development of micro-vascular complications in diabetes. The present study investigated the likelihood of inherent vasoactivity of C3R in itself, as well as its potential for protecting against MG-induced vascular abnormalities using isolated rat blood vessels. $\mathrm{C} 3 \mathrm{R}$ induced vasorelaxation dose-dependently in aortic rings (EC50: $2.43 \pm 0.57 \mathrm{M}$ ) pre-contracted with noradrenaline. These effects were endothelium-dependent and mediated primarily via nitric oxide (NO), as evidenced by the absence of relaxation in endothelium denuded preparations, as well as in the presence of $N^{\omega}$-nitro-L-arginine, an inhibitor of endothelial NO synthase (eNOS). The C3Rinduced vasorelaxation was also associated with a marked upregulation of eNOS mRNA expression, confirming the participation of NO in C3R's actions. Similarly, intravenous infusion of C3R (15-25 $\mathrm{mol} / \mathrm{kg}$ ) in anesthetized rats dose-dependently reduced mean arterial blood pressure ranging from $11-23 \%(p<0.05)$. Pre-treatment with MG $(500 \mathrm{M})$ led to vascular abnormalities, as observed by considerable potentiation $(p<0.05)$ of the vasoconstriction elicited by noradrenaline and impairment of vasorelaxation mediated by acetylcholine. Co-incubation with C3R ( $3 \mathrm{M})$ obliterated these abnormalities. Taken collectively, C3R possesses inherent vasoprotective properties that are effective against MG-induced vascular abnormalities, thus complementing its potential as a candidate antiglycation agent.

\title{
2.55. Physiological Properties of Tocotrienols: Evidence in Human Studies
}

Puvaneswari Meganathan and Ju-Yen Fu

Nutrition Unit, Product Development and Advisory Services Division, Malaysian Palm Oil Board, Malaysia

Vitamin E has been recognized as an essential vitamin since its discovery in 1922. Although the functions of tocopherols are well established, tocotrienols have been the unsung heroes of vitamin E. Due to their structural differences, tocotrienols were reported to exert distinctive properties compared to tocopherols. While most vegetable oils contain higher amount of tocopherols, tocotrienols were found abundantly in palm oil. Nature has made palm vitamin $E$ with up to $70 \%$ of total tocotrienols, among which alpha-, gamma-, and delta-tocotrienols are the major constituents. Recent advancements have shown their biological properties in conferring protection against cancer, cardiovascular diseases, neurodegeneration, oxidative stress, and immune regulation. Preclinical results of these physiological functions were translated into clinical trials gaining global attention. The biological activities exerted by tocotrienols will be reviewed in detail from the perspective of evidence reported in human studies to date in terms of efficacy, population, disease state, and bioavailability. This will serve as a platform to pave the future direction for tocotrienols in clinical settings.

\subsection{Chronic Stress and Suboptimal Nutrition Is Associated with Chromosome Instability in Dementia Family Carers}

\author{
C. F. Bull ${ }^{1,2}$, T. Almond ${ }^{1}$, H. Christensen ${ }^{3}$ and M. F. Fenech ${ }^{1}$ \\ CSIRO Health \& Biosecurity, Australia \\ 2 Molecular \& Biomedical Sciences, University of Adelaide, Australia \\ 3 Black Dog Institute, NSW, Australia
}

Telomere dysfunction and DNA damage lead to chromosomal instability and increased risk for cancers, as well as neurodegenerative and cardiovascular diseases. Chronic psychological stress is associated with shorter telomeres, while dietary micronutrient deficiency compromises DNA 
replication and genome integrity. We tested the hypothesis that chronically stressed dementia family carers have elevated DNA damage, and shorter telomeres relative to non-carer controls. Nutritional and stress status was determined in carers, and age and gender matched controls ( $n=42$ per group). Primary outcome measure was telomere length (TL). Secondary measures included perceived (PSS) and physiological stress (hair, serum, and saliva), biomarkers of chromosomal and epigenome stability, psychological factors, and nutritional and immune status. Carers had significantly higher perceived stress (PSS) $(p<0.0001)$ and anxiety $(p=0.0002)$ compared with controls. No differences were observed in TL or DNA damage; however, TL was negatively correlated with age $(\mathrm{r}=-0.5, p<$ $0.0001)$, homocysteine $(\mathrm{r}=-0.23, p=0.03)$, and alcohol intake $(\mathrm{r}=-0.3, p=0.006)$. Increased micronuclei (indicative of chromosome breakage/loss) were observed in carers with low serum zinc $(\mathrm{r}=-0.36, p=$ $0.02)$ and high serum cortisol $(\mathrm{r}=0.3, p=0.05)$. BDNF was lower in carers $(p=0.06)$, and inversely correlated with PSS $(\mathrm{r}=-0.33, p=0.06)$. IL6 was associated with lower cognitive function (MMSE) ( $\mathrm{r}$ $=-0.23, p=0.04)$, elevated MMA (risk factor for dementia) $(\mathrm{r}=0.27, p=0.01)$, and nucleoplasmic bridges (misrepair of broken chromosomes) $(r=0.25, p=0.03)$. While the data do not support the primary hypothesis, secondary measures indicate that chronic stress, and suboptimal nutrition, may interactively impact on chromosomal stability, contributing to increased morbidity, reduced immunity, and accelerated ageing observed in chronically stressed individuals.

\title{
2.57. Impact of AMY1 Gene Copy Number on Starch Digestion and Absorption
}

\author{
F. S. Atkinson 1,2 D. Hancock ${ }^{1}$, P. Petocz ${ }^{3}$ and J. C. Brand-Miller ${ }^{1,2}$ \\ 1 School of Life and Environmental Sciences, The University of Sydney, Sydney, NSW 2000, Australia \\ 2 Charles Perkins Centre, The University of Sydney, Sydney, NSW 2000, Australia \\ 3 Department of Statistics, Macquarie University, Sydney, NSW 2000, Australia
}

The salivary amylase gene (AMY1) shows remarkable variation in copy number (CN) in humans. AMY1 CN correlates with salivary alpha-amylase activity, but the physiological significance is uncertain. We explored the influence of AMY1 CN on starch digestion efficiency by assessing breath gas production following starch consumption. Fifteen individuals with low AMY1 and 15 with high AMY1 CN ( $3.9 \pm 0.9$ vs. $10.0 \pm 2.5$ copies, respectively, $p<0.001)$ completed two 8 -h test sessions in a randomised, crossover design. Each session consisted of a white rice meal, with or without added resistant starch, followed by a plain rice meal at $4 \mathrm{~h}$. Breath hydrogen and methane responses were assessed every hour and capillary blood samples were collected at regular intervals over $8 \mathrm{~h}$. Fasting breath hydrogen and total hydrogen production were similar in both groups. In contrast, both fasting breath methane $(12.8 \pm 2.5$ vs. $2.1 \pm 0.6 \mathrm{ppm}$, respectively, $p<0.001)$ and total methane production (5652 \pm 405 vs. $907 \pm 405$ ppm, respectively, $p<0.003$ ) were significantly higher in the low AMY1 group, irrespective of the meal type. Individuals with low AMY1 CN digested starch less efficiently, displaying lower postprandial glucose and insulin responses after consumption of rice meals and higher methane production in the large bowel. These findings suggest that having lower functional salivary amylase activity not only spares more carbohydrate for large bowel fermentation but also alters the balance of colonic microflora, which has potential implications for disease risk and gastrointestinal health.

\subsection{Relationship between UVR and Homocysteine Dependent on Genetic Variance in Folate Metabolism}

\author{
P. Jones ${ }^{1}$, E. Beckett ${ }^{1,2}$, Z. Yates ${ }^{3}$, M. Veysey ${ }^{4}$ and M. Lucock ${ }^{1}$ \\ 1 School of Environmental \& Life Sciences, University of Newcastle, Callaghan, NSW 2308, Australia \\ 2 School of Medicine \& Public Health, University of Newcastle, Callaghan, NSW 2308, Australia \\ 3 School of Biomedical Sciences \& Pharmacy, University of Newcastle, Callaghan, NSW 2308, Australia \\ 4 Hull-York Medical School, University of York, UK
}

One-carbon metabolism fuels multiple processes vital in health, including influencing disease risk via regulating homocysteine levels. These processes can be disrupted by independent or interactive effects of genetic and environmental factors. Evidence supports gene-environment interactions between several folate genes and an unavoidable environmental exposure, ultraviolet 
radiation (UVR). Methylenetetrahydrofolate dehydrogenase 1 (MTHFD1) codes for a multifunctional enzyme central to folate-mediated one-carbon metabolism, but has not yet been examined in this context. Aim of this study was to evaluate for interactions between the MTHFD1-G1958A variant and UVR on homocysteine levels. MTHFD1-G1958A, MTHFD1-T401C, MTHFR-C677T, SHMT-C1420T, and TYMS $28 \mathrm{bp} 2 \mathrm{R}>3 \mathrm{R}$ variants were genotyped via PCR/RFLP. Erythemal dose rate, a surrogate measure of UVR exposure, was assessed using satellite data (NASA's Total Ozone Mapping program). Interactions between these variables in influencing plasma homocysteine levels were assessed in a cross-sectional elderly cohort $(n=568)$. MTHFD1-G1958A independently predicted homocysteine level $(p=0.0367)$. Erythemal dose rate was negatively associated with homocysteine levels $(p<0.0001)$. A significant interaction was found between MTHFD1-G1958A genotype and UVR, with the relationship between UVR and homocysteine only significant in those carrying the polymorphic MTHFD1-G1958A allele $(p<0.0001)$. Multifactorial modelling with additional variants (MTHFR-C677T, SHMT-C1420T, TYMS 28 bp 2R > 3R, and MTHFD1-T401C) found MTHFD1G1958A was the only variant to significantly interact with erythemal dose rate to predict homocysteine levels ( pinteraction 0.0195). Findings support a key role of MTHFD1-G1958A in regulating homocysteine levels, as well as the relationship between UVR and homocysteine. These interactions are of potential health significance, due to the extensive involvement of one-carbon metabolism in disease processes.

\subsection{Habitual Coffee Consumption and Cognitive Function: A Mendelian Randomization Meta-Analysis in} up to 415,530 Participants

Ang Zhou, Amy E. Taylor, Ville Karhunen ${ }^{5}$, Yiqiang Zhan, Suvi P. Rovio, Jari Lahti, Per Sjögren, Liisa Byberg, Donald M. Lyall, Juha Auvinen, Terho Lehtimäki, Mika Kähönen,

Nina Hutri-Kähönen, Mia Maria Perälä, Michaëlsson, Anubha Mahajan, Lars Lind, Chris Power, Johan G. Eriksson, Olli T. Raitakari, Sara Hägg, Nancy L. Pedersen, Juha Veijola, Marjo-Riitta Järvelin, Marcus R. Munafò, Erik Ingelsson, David J. Llewellyn and Elina Hyppönen

School of Health Sciences, University of South Australia, Adelaide 5001, Australia

Coffee's long-term effect on cognitive function remains unclear with studies suggesting both benefits and adverse effects. We used the Mendelian randomization approach, and meta-analysed data from 10 European ancestry cohorts, including up to 415,530 participants and 300,760 coffee drinkers, to investigate the causal relationship between habitual coffee consumption and cognitive function in mid-to-later life. In each cohort, composite cognitive scores that capture global and memory cognition were computed using the available cognitive tests. A genetic score derived using CYP1A1/2 (rs2472297) and AHR (rs6968865) was chosen as a proxy for habitual coffee consumption. Null associations were observed when examining the associations of the genetic score with global and memory cognition ( $\beta=-0.0007,95 \%$ C.I. -0.009 to $0.008, p=0.87 ; \beta=-0.001,95 \%$ C.I. -0.005 to $0.002, p=0.51$, respectively), with high consistency between studies (Pheterogeneity $>0.4$ for both). Domain specific analyses using available cognitive measures in the UK Biobank also did not support effects by habitual coffee intake for reaction time, pairs matching, reasoning, or prospective memory $(p \geq$ 0.05 for all). Despite the power to detect very small effects, our meta-analysis including 300,760 coffee drinkers provided no evidence for causal long-term effects of habitual coffee consumption on memory function or global cognition.

\subsection{Causal Relationship between BMI and Depression: A Mendelian Randomisation Study}

A. Mulugeta ${ }^{1}$, J. Tyrrell ${ }^{2}$, A. Zhou ${ }^{1}$, T. Frayling ${ }^{2}$ and E. Hyppönen ${ }^{1}$

1 Centre for Population Health Research, University of South Australia, Adelaide, Australia

2 Exeter Medical School, Exeter, UK

Obesity is associated with depression, but evidence for underlying causality is uncertain. We used Mendelian randomization (MR) approach and information from up to 407,277 UK Biobank participants to test the hypothesis that higher BMI increases the risk of major depressive disorders 
(MDD). MDD was determined based on self-reported and hospital episode data. A weighted genetic risk score (GRS) using 73 SNPs was constructed to approximate differences in BMI based on previously published genome-association analysis. Separate risk scores were prepared for genetic variants affecting BMI using different biological mechanisms. Phenotypic association between higher BMI and MDD was noted for both genders, with somewhat strong association seen in women compared to men (OR 1.24, 95\% CI 1.23-1.26, vs. 1.14; 1.12-1.16, $p<1.4 \times 10^{-32}$ for both). BMI GRS, and all mechanism specific GRSs, showed the expected associations with BMI ( $p<2.3 \times 10^{-61}$ for all). MR analyses provided some support for a causal relationship between BMI and depression, with evidence stronger for women than for men (OR per genetically instrument BMI unit 1.30, 95\% CI 1.15-1.47, $p=3.2 \times 10^{-5}$; OR 1.11, 0.94 to 1.32, $p=0.2$ ). Exploration for the association between mechanism-specific GRS and MDD showed an association for variants implicated in neurodevelopment $\left(p=1.4 \times 10^{-5}\right)$, hypothalamic expression $(p=0.006)$, and cell cycle regulation $(p=$ 0.003) but less so for GRSs reflecting other pathways. This large-scale study provides tentative evidence for a causal association between higher BMI and depression risk, especially in women.

\title{
2.61. The Medical School Experience - How Does It Affect Attitudes towards Nutrition Care?
}

S. Martin 1, E. Sturgiss ${ }^{1}$, L. Ball ${ }^{2}$ and K. Douglas ${ }^{1}$

1 Academic Unit of General Practice, Australian National University Medical School, Australia

2 Menzies Health Institute Queensland, Griffith University, Australia

Healthy nutrition is important in the prevention and management of chronic disease, with doctors centrally placed to provide nutrition care. Despite this, studies have reported a lack of effectiveness of nutrition advice from doctors to patients in promoting behavioural change. To address this, there have been efforts to increase the explicit teaching of nutrition to medical students. We want to explore how medical students experience nutrition education and are interviewing ANU medical students using "gamified" case studies and semi-structured interviews. Using inductive thematic analysis, we are exploring the factors that have an impact on the student experience and shape their ideas about nutrition. Emerging themes include the view that nutrition care is a core skill that all doctors should have, but that it is implied and peripheral in their learning. Medical students perceive that they have a good level of nutrition knowledge, as it is something you can learn in places other than medical school. There is a tension between seeing general nutrition advice as "obvious" and easy to provide, compared to specific nutrition care required for particular medical conditions. Doctors are seen as managing multiple issues under time pressure, with dietitians seen as specialists that can spend extra time with patients. The modelling of nutrition care by clinician teachers is variable, emphasized in some settings but devalued in others. This study makes an important contribution to understanding the experience of nutrition education in medical school. It will contribute positively to efforts to promote nutrition care as an essential clinical skill.

\subsection{The Impact on Assessment Tasks of Embedding Nutrition into Medical Course Curriculum at Deakin University}

\author{
R. Perlstein ${ }^{1}$, J. McCleod ${ }^{2}$, N. Stupka ${ }^{2}$, C. Bell ${ }^{2}$, S. McCoombe ${ }^{2}$ and C. Nowson ${ }^{1}$ \\ 1 School of Exercise and Nutrition, Deakin University, Australia \\ 2 School of Medicine, Deakin University, Australia
}

Background/Aims: One of the key drivers of learning is assessment. Staff within the Schools of Exercise and Nutrition Sciences and Medicine have been working collaboratively to embed nutrition into the medical curriculum. The aim of this study was to assess the proportion of nutrition questions included in the summative exams administered within the Bachelor of Medicine Bachelor of Surgery. Methods: Year 1 and 2 exams contributed between 63\% (2013)-68\% (2016) of the summative assessment, whilst in year 3/4 they made up 38\% (2013-16). The exams contained both multi choice (MCQs) and short answer questions (SAQs). The questions that included some nutrition content were classified as wholly related to nutrition (WN) or partially related to nutrition (PN), where nutrition 
was a component. Results: The total number of exam questions in each year ranged from 1032 to 1274 . There was a trend for an increase in the proportion of nutrition-related questions over the years: MCQs: $2013-0 \%$ (39\% WN, 61\% PN), 2014-8\% (55\% WN, 45\% PN), 2015-6\% (58\% WN, 42\% PN), 2016-10\% (42\% WN, 58\% PN), SAQs: $2013-12 \%$ (50\% WN, 50\% PN), $2014-22 \%(70 \% \mathrm{WN}, 30 \%$ PN), 2015-17\% (33\% WN, 67\% PN), and 2016-19\% (47\% WN, 53\% PN). Conclusions: Nutrition was represented in both multiple choice and short answer questions with an overall increase in the nutrition content between 2013 and 2016. This is likely to be due to the increased collaboration and involvement by nutrition teaching staff with medical educators since 2009.

\subsection{The Effects of NIPRCES on Nutrition-Related KAP: A Comparison between Two nearby Counties in China}

\section{Y. Xu and G. Ma}

Department of Nutrition and Food Hygiene, School of Public Health, Peking University, Beijing, China

The National Improvement Program for Rural Compulsory Education Students (NIPRCES) in China started from November, 2011, which aimed to improve the nutritional status of rural students. By 2016, 183 billion CNY had been invested into the program, which covered more than 33.6 million rural students nationwide. However, little research has been conducted to compare students' nutrition-related knowledge, attitudes, and practices (KAP) between subsidized and unsubsidized areas. Data on nutrition-related KAP were collected by self-administered questionnaires among rural students of four random chosen compulsory schools, aged from grade 4 to grade 9 , in two nearby counties in China. One county was selected as pilot of NRPICES, whose students had been receiving subsidies for their school meals (breakfast) since 2012, while students in another county were unsubsidized. The breakfast quality was assessed by food variability. Totally, 378 and 469 students were selected in the subsidized and unsubsidized county, respectively. Compared with students in the unsubsidized area, students in NIPRCES had better nutritional knowledge $(p<0.001)$, more positive attitudes towards nutrition $(p<0.001)$, higher breakfast frequencies $(p<0.05)$, and better breakfast qualities $(p<0.001)$. Moreover, consumptions of meat, milk, eggs, beans, and fresh vegetables were more frequent among subsidized students $(p<0.05)$. The study indicates a promoting effect of NIPRCES on nutrition-related KAP of rural students. Further evaluations are required to confirm the potential benefits of NIPRCES.

\subsection{Greener Groceries: Insights into Australians' Views on Sustainable Food Choices 2010-2017}

\section{A. S. Lawrence and H. J. Jones}

Dairy Australia, Level 5, IBM Centre, 60 City Road, Southbank, Victoria, Australia

In 2010, the United Nations Food and Agriculture Organisation defined sustainable diets as those that are "protective and respectful of biodiversity and ecosystems, culturally acceptable, accessible, economically fair and affordable; nutritionally adequate, safe and healthy; while optimizing natural and human resources." The purpose of this study was to examine attitudes, perceptions, influences, and behaviour in relation to food choice and sustainability among adults living in Australia between 2010 and 2017. Eight annual on-line surveys of the general adult population $(n=1621-1856)$ were undertaken. Weighting ensured gender and age representativeness. Independent samples $t$-tests determined differences. Since 2010, the importance of food being produced in an environmentally responsible way has steadily risen in prominence as a factor influencing food choice (in 2010,58\% said it was critical, very important, or important compared with $67 \%$ in $2017, p<0.01$ ). In contrast, the proportion of adults agreeing that the dairy industry is a significant contributor to greenhouse gas emissions was unchanged. In June 2017, 40\% of adults reported having changed what they eat and drink in order to be more sustainable. The main reported dietary changes were decreased consumption of soft drink, meat, sugar, processed foods, and packaged foods, and increased consumption of vegetables, fruit, water, protein, and nuts/seeds/grains. Respondents indicated the key influencers of their sustainable food choices were 
health concerns, environmental concerns, and the media. In conclusion, whether food is produced in an environmentally responsible way is becoming an increasingly important determinant of food choice among adults living in Australia. Funding source: Dairy Australia.

\subsection{Improving Health Outcomes of Indonesians through Building Capacity of the Nutrition Workforce in} Yogyakarta

D. C. Sulistyoningrum ${ }^{1}$, K. M. Dickinson ${ }^{2}$, L. Matwiejczyk ${ }^{2}$, T. Sudargo ${ }^{1}$ and J. Miller ${ }^{2}$

1 Department of Nutrition and Health, Faculty of Medicine Universitas Gadjah Mada, Yogyakarta, Indonesia

2 Discipline of Nutrition and Dietetics, College of Nursing and Health Sciences, Flinders University, Adelaide, SA, Australia

Dietitians and nutritionists working in academic, clinical, and community settings in Yogyakarta, Indonesia identified projects that addressed the nutrition needs of disadvantaged groups in their communities. With the support of an Australia Award Fellowship, a formal professional development program was hosted by Flinders University in Adelaide, Australia during February-March 2017 for 15 Indonesian Fellows. The program consisted of field work, course work, and formal mentoring from local experts. Upon completion of the program, Fellows returned home to progress their chosen project. Outcomes have included establishing a dietetic internship program commencing in September 2017, a student-led dietetic outpatient clinic that received a competitive support grant from the University, and inter-sectoral program endorsement plus funding from the Department of Women Empowerment, Child Protection, and Planned Parenthood of Sleman District, Yogyakarta for the nutrition school children program. In addition, the Ayah Asih breastfeeding project was implemented and received enormous engagement from the community. Clinical projects for paediatric, cancer, and mental health patients received support from their respective hospitals. In April 2017, mentors provided in-country support with a follow up visit to Yogyakarta to monitor projects' progress. This experience showcases a successful mentoring partnership between Australian nutrition and dietetic professionals and Indonesian counterparts. Strong people-to-people links supported by the Australia Awards Fellowship have shaped a foundation for strengthening the nutrition and dietetics workforce and improving the health outcomes of people in Yogyakarta. Ongoing mentoring is required to ensure implementation and success of projects.

\subsection{Changes in Body Weight and Nutritional Status in South Australian Nursing Home Residents}

T. Arjuna ${ }^{1,2}$, N. Luscombe-Marsh 1,3, K. Lange ${ }^{1}$, A. I. Kang 4, P. Hickman ${ }^{4}$, C. Edwards ${ }^{4}$, S. Reid ${ }^{4}$, I. Chapman ${ }^{1}$ and S. Soenen ${ }^{1}$

1 Discipline of Medicine and National Health and Medical Research Council (NHMRC) Centre of Research Excellence in Translating Nutritional Science to Good Health, The University of Adelaide, Adelaide, SA 5005, Australia

2 Department of Health Nutrition, Faculty of Medicine, Universitas Gadjah Mada, Yogyakarta, Indonesia

3 CSIRO Nutrition and Health Program, Health and Biosecurity Business Unit, Adelaide, Australia

4 Southern Cross Care (South Australia \& Northern Territories) Inc., Australia

Ageing-related changes in body weight and composition impact quality of life of older people. Therefore the aim of the retrospective study was to determine body weight, body mass index (BMI), nutritional status assessed by malnutrition universal screening tool (MUST), pain, and length of stay of a cohort of elderly nursing home residents in Adelaide, South-Australia, as well as the factors associated with changes in body weight over 6-12 months. 1020 residents aged $87 \pm 8$ years were in the 6-months retrospective analysis, and a subset of 752 residents in the 12-months sub-group. The average weight and BMI for the overall cohort were $66 \pm 16 \mathrm{~kg}$ and $25 \pm 6 \mathrm{~kg} / \mathrm{m}^{2}$. Almost $30 \%$ of residents were at medium or high nutritional risk (14\% and $16 \%$ ). Body weight decreased $0.4 \pm 4.1 \mathrm{~kg}$ $(0.5 \pm 6.4 \%)$ over 6 -months $(p=0.006)$ and $0.9 \pm 5.2 \mathrm{~kg}(1.3 \pm 7.8 \%)$ over 12 -months $(p<0.001) .46 \%$ of residents had marked weight change ( $\geq 5 \%$ loss or gain) over 12 -months. Residents in the lowest BMI tertile $\left(\leq 23 \mathrm{~kg} / \mathrm{m}^{2}\right)$ were most likely to experience both marked weight change $(52 \%)$ and weight reduction $(30 \%)$. Weight loss was associated with higher pain scores $(p=0.012)$ and greater length of 
stay in the nursing home $(p=0.002)$. These findings indicate that, on average, these older people lost weight, with high rates of both substantial weight loss and gain, particularly among those in the lowest BMI tertile. Almost a third in the lowest BMI tertile lost $5 \%$ or more body weight, putting them at increased risk of undernutrition-related morbidity, suggesting greatest attention to prevent and treat such morbidity should be focused on that group.

\subsection{Nutritional Status, Eating Pattern, and Physical Activity as Risk Factor of Prediabetes among Adult in} Yogyakarta

Susetyowati, Emy Huriyati, B. J. Istiti Kandarina and Farah Faza

Departmenet of Nutrition and Health, Faculty of Medicine, Universitas Gadjah Mada, Yogyakarta 55281, Indonesia; susetyowati@ugm.ac.id (S.)

Background: Diabetics in Indonesia increase from 1.1\% (2007) to 2.1\% (2013). This is preceded with pre-diabetes whose risk factors are obesity, unbalanced diet, and sedentary lifestyle. Objectives: determining nutritional status, eating pattern, and physical activity that may affect pre-diabetes among adults. Methods: Cross-sectional study and subjects represent the most populated area in Yogyakarta, taken in Umbulharjo (urban), Turi, and Minggir (rural) by cluster sampling. We interviewed subjects using food consumption pattern questionnaire, measured nutritional status: body mass index/BMI, waist circumference/WC, body fat mass, Global Physical Activity Questionnaire/GPAQ, and fasting blood glucose $(<100 \mathrm{~g} / \mathrm{dL}$ was normal and $>100 \mathrm{~g} / \mathrm{dL}$ was prediabetes). Results: From 385 subjects, 162 (42.1\%) were pre-diabetes and 223 (57.9\%) were normal, with prevalence in urban $(67.28 \%)$ higher than rural $(32.72 \%)$. BMI and visceral fat were higher on pre-diabetes than normal ( 23.96 vs. $23.57,8.34$ vs. $7.03, p=0.44,0.01$, respectively). WC was higher on pre-diabetes than normal ( 80.89 vs. 78.78 on male and 82.72 vs. 79.54 on female, $p=0.20$ and 0.05 ). Sweet, fatty, grilled, and processed food; instant noodles; and physical activity were associated with pre-diabetes $(p \leq 0.001)$. There was no association between total fruit and vegetable consumption with pre-diabetes $(p=0.27)$. Conclusion: Visceral fat, food consumption pattern, and physical activity are risk factors of pre-diabetes. For prevention risk of pre-diabetes, we should control nutritional status in normal category and minimize unhealthy food consumption.

\subsection{Relationships between Nutritional Status, Cognitive Impairment, and Functional Capacity: Findings from a Cross-Sectional Study of Indonesians Aged 65 or Older}

\section{T. Arjuna ${ }^{1,2}$, S. Soenen ${ }^{1}$, R. A. Hasnawati ${ }^{2}$, K. Lange ${ }^{1}$, I. Chapman ${ }^{1}$ and N. D. Luscombe-Marsh ${ }^{1,3}$}

1 Discipline of Medicine and National Health and Medical Research Council (NHMRC) Centre of Research Excellence in Translating Nutritional Science to Good Health, The University of Adelaide, Adelaide, SA 5005, Australia

2 Department of Nutrition and Health, Faculty of Medicine, Universitas Gadjah Mada, Yogyakarta, Indonesia

3 CSIRO Nutrition and Health Program, Health and Biosecurity Business Unit, Adelaide, Australia

Malnutrition is a global health priority, particulalry in developing countries including Indonesia because of the dual-burden of high birth rates and population ageing. However, there remains a paucity of knowledge around the impact of poor nutrition (both malnutrition and at-risk of malnutrition) on health, including the nutritional status and cognitive and physical capacity of Indonesians aged 65 years and older. This study aimed to measure these outcomes in communitydwelling older adults living in rural and urban areas of Yogyakarta Indonesia, and determine the relationships between nutritional status and cognitive with physical capacity. A cross-sectional study involving 527 older individuals aged $\geq 65$ years was conducted. Health outcomes were self-reported using structed questionnaires; nutritional status was determined using anthropometric measures and the Mini Nutritional Assessment (MNA); cognitive function was determined using the Mini Mental State Examination (MMSE); and functional capacity was determined from measurements of grip strength, gait speed, and Instrumental Activities of Daily Living (IADLs). Key findings were that rural compared with urban participants had poorer nutritional status, a lower lower cognitive function, and grip strength, but faster gait speed while being more dependent on assistance to 
perform daily activities (all $p<0.05$ ). Cognitive function, rather than nutritional status, has the strongest association with functional capacity (correlation coefficient with grip strength, gait speed, and IADL was $0.446,0.332$, and -0.457 , respectively, all $p<0.001$ ). These findings indicate that the development of strategies to improve cognitive function and nutritional status is of pivotal importance for the wellbeing of Indonesians citizens.

\subsection{Hospital Staffs' Perceptions of a New Technology to Engage Patients in Their Nutrition Care at the Bedside}

S. Roberts ${ }^{1}$, A. Marshall ${ }^{1,2}$ and W. Chaboyer ${ }^{1}$

1 National Centre of Research Excellence in Nursing, Menzies Health Institute Queensland, Griffith University, Australia

2 Gold Coast Health, Gold Coast, Queensland 4207, Australia

Optimal nutrition is vital for health and recovery during hospitalisation; however, many patients do not meet their nutritional needs in hospital. Active patient involvement, or participation, in their nutrition care can improve dietary intakes. Our team is developing a program by which patients can participate in their nutrition care at the bedside. As end-user feedback is vital for ensuring feasible, acceptable, and sustainable interventions, this study explored hospital staff's perceptions of this technology. Interviews were conducted with hospital staff providing routine nutrition care to patients (dietitians, nutrition assistants, doctors, nurses, and foodservices). Interviews were recorded, transcribed, and analysed using thematic analysis. Staff expressed positive views of the program and wanted it implemented. Their responses formed three themes and various sub-themes: (1) enacting patient participation in practice; (2) optimising nutrition care; and (3) considerations for implementing the program in practice. Staff thought the program would improve various aspects of nutrition care and enable patient participation. They recognised benefits to the program for patients and staff, and hence found it acceptable. Characteristics of the program itself and allocation of roles and responsibilities in operationalising it were seen as pivotal for success. Whilst staff raised some concerns, they focused on overcoming barriers and facilitating implementation if the program were to be adopted in practice.

\subsection{Effect of the Mediterranean Diet on Cognition and Brain Morphology/Function: A Systematic Review and Meta-Analysis of RCTs}

S. Radd-Vagenas ${ }^{1}$, S. L. Duffy ${ }^{2,3}$, S. L. Naismith ${ }^{3,4}$, B. J. Brew ${ }^{5,6}$, V. M. Flood ${ }^{1}$ and M. A. Fiatarone Singh 1,7

1 Faculty of Health Sciences, The University of Sydney, Australia

2 Charles Perkins Centre \& Woolcock Institute of Medical Research, Central Clinical School, Faculty of Medicine, The University of Sydney, Australia

3 Healthy Brain Ageing Program, Brain and Mind Centre, The University of Sydney, Australia

4 Charles Perkins Centre, School of Psychology, Faculty of Science, The University of Sydney, Australia

5 Faculty of Medicine, University of New South Wales and Department of Neurology, Australia

6 St Vincent's Hospital and Peter Duncan Neurosciences Unit for Applied Medical Research, NSW, Australia

7 Hebrew SeniorLife and Jean Mayer USDA Human Nutrition Research Center on Aging at Tufts University, USA

Currently no medical treatment exists to prevent, delay, or modify the course of dementia, but reviews of primarily observational studies suggest a Mediterranean diet (MedDiet) may benefit cognition and reduce dementia risk. We searched nine databases (inception to July 2015) to identify randomised controlled trials (RCTs), which tested a MedDiet compared to an alternate diet for cognitive or brain morphology/function outcomes, with additional focus on intervention diet quality and its relation to the 'traditional' Mediterranean dietary pattern. Analyses were based on 66 cognitive tests and one brain function outcome from five included studies ( $\mathrm{n}=1888$ participants). Prescribed MedDiets varied considerably between studies, particularly in quantitative food advice. Only $12.1 \%$ of individual outcomes (8/66) at trial level significantly favoured a MedDiet diet for 
cognitive performance, with effect sizes (ESs) ranging from small (0.32) to large (1.66), whereas two favoured control. Data limitations precluded a meta-analysis. However, 3/8 domain composite cognitive scores (memory, frontal, and global function) were significant, with ESs ranging from 0.39 to 1.29. Risk of low plasma, Brain-derived Neurotrophic Factor (BDNF) was reduced (OR $=0.22 ; 0.05$ to 0.90 ) in those on a MedDiet in a post-test comparison at 3 years, and genetic profile appeared predictive of beneficial adaptations to a MedDiet for some cognitive outcomes at 6.5 years in PREDIMED (Prevenciòn con Dieta Mediterránea). There was no benefit for incident cognitive impairment or dementia. Although presently available data are mostly non-significant, with small ESs, the significant improvements in cognitive domain composites in the most robustly designed study warrant additional research.

\subsection{Oral and Extra-Oral Sweet Receptor Expression in a Model of Antibiotic Induced Dysbiosis}

E. L. Beckett ${ }^{1}$, G. Burns ${ }^{2}$, M. Lucock ${ }^{3}$, M. Veysey ${ }^{1,4}$ and S. Keely ${ }^{2}$

1 School of Medicine and Public Health, University of Newcastle, Callaghan 2308, Australia

2 School of Biomedical Sciences and Pharmacy, University of Newcastle, Callaghan 2308, Australia

3 School of Environmental and Life Sciences, University of Newcastle, Callaghan 2308, Australia

4 Hull York Medical School, UK

Antibiotic treatment is associated with weight gain and risk for obesity. The mechanisms driving this association are not yet clear; however, dysbiosis has been identified as a potential causative factor in weight gain and obesity. Procedures that impact diet, weight, and gastrointestinal microbiota, such as gastric banding surgery, have been linked to modified expression of TAS1R taste receptors on the tongue. TAS1Rs are also expressed in the gastrointestinal tract, and so may be involved in detecting sweet compounds and appetite control. We assessed how antibiotic-induced dysbiosis influenced expression of TAS1R2 and TAS1R3, the two components of the heterodimeric sweet taste receptor, on the tongue and in the colon in a mouse model. Mice (8/group) received amoxicillin and clavulanate (5 day course), or control treatment. 3 days post-treatment, colon and tongue tissues were collected, RNA was isolated (TRIZOL), and expression of TAS1R2 and TAS1R3 was assessed by qPCR. Expression of TAS1R2 was decreased in colon $(1.0 \pm 0.29$ vs. $0.37 \pm 0.07$ relative expression units (REU), $p=0.048)$ and tongue ( $1.0 \pm 0.34$ vs. $0.14 \pm 0.03 \mathrm{REU}, p=0.026)$ tissue of antibiotic treated mice. However, TAS1R3 expression was not significantly different following antibiotic treatment (Colon: $1.0 \pm 0.13$ vs. $1.10 \pm 0.42 \mathrm{REU}, p=0.81$; Tongue: $1.0 \pm 0.28$ vs. $1.05 \pm 0.36 \mathrm{REU}, p=0.92$ ). Reduced expression of TAS1R2 following antibiotic treatment may reduce sensation of sweet dietary compounds on the tongue and reduced enteroendocrine signaling in the colon, altering appetite control. Future studies are required to assess the duration of effects, the influence on diet and weight, and how altered mRNA expression translates into changed protein expression levels.

\subsection{Potential Efficacy of Probiotics from Different Origins}

\section{R. Vemuri ${ }^{1}$, R. Eri, ${ }^{1}$, M. J. Ball ${ }^{1,2}$, S. Tristram ${ }^{1}$ and K. D. K. Ahuja ${ }^{1}$ \\ 1 School of Health Sciences, University of Tasmania, Launceston, TAS, Australia \\ 2 School of Health \& Biomedical Sciences, RMIT University, VIC, Australia}

To be efficacious, probiotics must exhibit functional characteristics including the ability to withstand gastric acidity and adhere to intestinal epithelium and immunomodulate. Although there are numerous in vitro studies showcasing the potential of different probiotics, none have compared probiotics of different origins. We investigated the effects of different origin bacterial strains on adhesion capacity and release of inflammatory markers with human colonic cells (HT-29 and LS$174 \mathrm{~T}$ ) and the survivability in a static in vitro digestion model in the presence of gastric and intestinal enzymes involved in digestion. $10^{\circ}$ Colony Forming Units (CFU)/mL of Lactobacillus acidophilus DDS1 (human origin), Bifidobacterium lactis UABla-12 (human origin), L. plantarum UALp-05 (plant origin), and Streptococcus thermophilus UASt-09 (dairy origin) were used for these experiments. All strains survived in the range of $10^{6}$ to $10^{8} \mathrm{CFU} \mathrm{mL}^{-1}$ in presence of $\mathrm{pH} 2.5$, bile, and digestive enzymes such as pepsin and pancreatin. Adhesion and survivability for all strains ranged from 77 to $95 \%$. All the 
strains downregulated pro-inflammatory IL-8 and upregulated anti-inflammatory IL-10 levels. $L$. acidophilus DDS-1 showed the highest survivability, adhesion, and immunomodulatory potential. This in vitro evaluation data suggests that all the tested strains have the potential to be effective probiotics.

\subsection{Targeting Gut Microbiota: Lactobacillus Alleviated Type 2 Diabetes via GRP43/GLP-1 \&PYY Pathway}

Y. Shan, P. Lei, B. Li and K. Li

Harbin Institute of Technology, China

Our previous findings show that G15 and Q14, two species of lactobacillus from Chinese traditional fermented diary food, have potential in the prevention of diabetes. Herein, we clarified their gut microbiota-related mechanisms. G15 and Q14 lowered the blood glucose, insulin, and glucagon concentrations in blood using ELISA detecting kit. Intestinal flora analysis by quantitative real-time PCR indicated that G15 and Q14 significantly shortened the number of G- Bacteroides and Enterobacterium, which subsequently decreased the levels of LPS and inflammatory factors in the blood. Quantities of various short-chain fatty acids (SCFAs) in the feces by gas chromatography technology showed that G15 and Q14 enhanced the concentrations of acetic acid and butyric acid, which were degraded by the intestinal flora including Lactobacillus, Bifidobacterium, Clostridium leptum, and Bacteroides prevotella. The increased levels of acetic acid and butyric acid stimulated the transcriptional expression of G-protein uncoupled receptor 43 (GPR43), not GPR41 in the colon, followed by the release of GLP-1 and PYY into the blood detected by ELISA method. G15 and Q14 obviously improved the disorganization of the intestinal mucous according to the pathological observation of HE staining. Two tight conjuction proteins including ZO-1, occuludin, and not claudin were significantly upregulated by G15 and Q14, which are partly responsible for the re-organization of the intestinal permeability and mucosal barrier integrity. Lactobacillus G15 and Q14 alleviated type 2 diabetes (T2D) in a gut microbiota-dependent way via downregulating Gram-negative, bacteriarelated LPS secretion, as well as upregulating SCFAs-producing, bacteria-related, G protein-coupled receptor 43 (GRP43) pathway.

\subsection{Weight Loss and Improved Small-Intestinal Permeability in Overweight Individuals Following a Meal Replacement Program: A Randomized Controlled Trial}

\section{D. Tran, K. Nyland, M. A. Rebuli, J. Bowen and M. Noakes}

CSIRO Health and Biosecurity, Adelaide, SA 5000, Australia

Increased intestinal permeability has been demonstrated in morbidly obese individuals leading to translocation of endotoxins, which in turn may promote low-grade inflammation. The relationship between weight loss, small intestinal permeability, and inflammation is not clear. The aim of the present study is to evaluate the effect of weight loss following a meal replacement program (MRP) on intestinal permeability in overweight and obese individuals. Adults aged 25-60 years with a BMI $\geq 27 \mathrm{~kg} / \mathrm{m}^{2}$ and no chronic illness were recruited in Adelaide, South Australia. Study participants ( $\mathrm{n}=$ 164) were randomised to a 16 week program of either a standard MRP (S-MRP; $n=82$ ) or a modified intermittent fasting MRP (M-MRP, $n=82)$. All participants were provided a meal plan based on their individual energy requirements. Weight, small intestinal permeability, and C-reactive protein (CRP) was assessed at baseline and after 16 weeks of weight loss. Both diets showed a significant reduction in weight after the 16 week program (M-MRP; $-11.2 \pm 0.6 \mathrm{~kg}$, S-MRP; $10.7 \pm 0.5 \mathrm{~kg}$, NS between treatments). Compared to baseline (lactulose:rhamnose ratio S-MRP $8.81 \pm 0.74, \mathrm{M}-\mathrm{MRP} 8.84 \pm 0.67$ ), both groups showed improved permeability after the 16 week program (lactulose:rhamnose ratio SMRP $6.6 \pm 0.70$; M-MRP $5.6 \pm 0.57$ ). By week 16, there was also a significant reduction in CRP from baseline values, independent of treatment (S-MRP $-0.45 \pm 0.16 \mathrm{mg} / \mathrm{L} ; \mathrm{M}-\mathrm{MRP}-0.59 \pm 0.23 \mathrm{mg} / \mathrm{L} p<$ $0.05)$. Both meal replacement-based strategies were associated with improved intestinal permeability, CRP, and weight loss in overweight and obese adults. 


\subsection{Global Metabolite Profiling of Multiple Sample Types to Identify Markers for Functional} Gastrointestinal Disorders

K. Fraser ${ }^{1,2,3}$, N. C. Roy ${ }^{1,2,3,4}$, H. Stirrat ${ }^{1}$, W. Young ${ }^{1,2}$ and R. Gearry ${ }^{2,4}$

1 Food Nutrition \& Health Team, AgResearch Grasslands, Palmerston North, New Zealand

2 The High-Value Nutrition National Science Challenge, New Zealand

3 Riddet Institute, Massey University, Palmerston North, New Zealand

4 University of Otago, Christchurch, New Zealand

Irritable Bowel Syndrome (IBS) is a functional gastrointestinal (GI) disorder characterised by chronic or recurrent abdominal discomfort mostly associated with changes in GI habit in the absence of a detectable organic cause. Global metabolite profiling across multiple sample types can provide biochemical fingerprints that may assist with understanding the underlying mechanisms. In a casecontrol study, we aimed to identify microbial and host factors in plasma to provide mechanistic insights into functional GI disorders and increase the predictability of phenotypes for use in nutrition intervention studies. Individuals with functional GI symptoms (cases) or asymptomatic (controls) undergoing colonoscopy were recruited at Christchurch hospital, and a subset of 102 plasma samples and 70 breath samples were measured. Plasma samples were subjected to biphasic extraction and liquid chromatography mass spectrometry analysis (LC-MS), while breath volatiles were captured on carbon sorbent and subsequently analysed by gas chromatography mass spectrometry (GC-MS). LC-MS analysis detected 491 polar, 458 semi-polar, and 768 non-polar (lipid) metabolite features in plasma, while GC-MS profiling yielded 37 volatile components in the breath. Plasma metabolomic profiles differed significantly between the IBS phenotypes and the control subjects, with major perturbations observed in amino acid, bile acid, and lipid metabolism. Breath volatile profiles revealed weak separations between the IBS phenotypes. Metabolomics can detect and determine differential metabolites and pathways affected between phenotypes, and the next steps include applying a systems biology approach using metabolomics (breath, plasma, and faeces) datasets of a larger cohort size to identify key pathways and biomarkers.

\subsection{Development of Nutrition Science Competency Standards for Australian Undergraduate Nutrition Science Degrees: A Joint NSA and ANT Initiative}

T. Lawlis, S. Torres, A. Coates, K. Clark, K. Charlton, L. Wood and A. Devine

University of Canberra, Canberra, ACT 2600, Australia

The need for updated competency standards for nutrition scientists was identified at a cobadged Australian Nutrition Trust/Nutrition Society of Australia (NSA) workshop on Curriculum for Nutrition Science degrees, at the NSA Annual Scientific Meeting, in November 2014. An iterative multiple methods approach comprising three stages was undertaken: (1) scoping study of existing competencies; (2) exploratory survey; and (3) a modified Delphi process (completed in 2 rounds) involving 128 nutrition experts from industry, community, government, and academia. A $\geq 70 \%$ consensus rule was applied to findings in Rounds 1 and 2 of the Delphi process in order to arrive at a final list of competency standards. Overall, there was a high level of consensus on the proposed competency standards. The revised competency standards comprise five core competency areas and three specialist competency areas. The five core competency standards are underpinned by fundamental knowledge, skills, attitudes, and values and include the following areas required for practice: Nutrition Science, Food and the Food System, Nutrition Governance, Sociocultural and Behavioural Factors, Nutrition Research and Critical Analysis, and Communication and Professional Conduct. The three specialist competency areas are Food Science, Public Health Nutrition, and Animal Nutrition. The new nutrition science competency structure provides a framework against which to benchmark current and future nutrition science degrees in Australia and is expected to lead to improved employability skills of nutrition science graduates. These revised competencies for the contemporary role of nutritionists and nutrition scientists will further build professional identity in the changing landscape of nutrition. 


\subsection{Effectiveness of Multiple Micronutrient Powder on Anemia and Growth among 8-10-Month-Old Children in Low SES Community JAKARTA}

\section{S. Bardosono, R. Sekartini, D. Chandra, T. Wiguna, T. Sundjaya and J. Bindels}

University of Indonesia, Barat 16424, Indonesia

Background: Anaemia and stunting are major public health concerns for Indonesian young children aged 6-36 months. Only limited information is available from studies with multimicronutrient supplements in Indonesia. Methods: A double-blinded randomized placebo controlled study was done with 232 subjects. Multi-micronutrient powder sachets for addition to complementary foods were provided to 140 subjects while 92 subjects obtained placebo sachets. Inclusion was at age 8-10 months; intervention duration was 12 months. Outcome parameters were anaemia, haemoglobin and ferritin level, growth, and feeding practices. The study was done in a low SES community in Jakarta (2014-2016). Results: Per-protocol analysis in 189 subjects revealed that anaemia prevalence, haemoglobin, serum ferritin levels, and growth did not change significantly upon intervention within groups or between groups. Dietary intake assessed at baseline, 6 months intervention, and endline revealed major differences between groups for breastfeeding rate, intake of flesh foods, dairy products, and dietary diversity. Sub-group analysis in 126 children still breastfed at 6 months intervention showed a strongly improved match of dietary intake between both groups ( $n=36$ and 90, respectively), except for vitamin A-rich fruits and vegetables. Height-for-age Z-score decreased particularly in the control group and was significantly lower at endline compared to the intervention group $(p=0.039)$. There was no significant difference in the changes of haemoglobin, but ferritin level decreased significantly lower in the control group $(p=0.034)$. Conclusion: Multimicronutrient powder provision to breastfed young children aged 8-22 months is effective in preventing iron deficiency and stunting in low SES communities.

\subsection{Is There Iodine Deficiency among Pregnant Women in the South Central Coast of Vietnam?}

\section{T. Bui ${ }^{1}$, P. Winichagoon ${ }^{2}$ and S. Gowachirapant ${ }^{2}$}

1 International University, Vietnam National University-HCMC, Hanoi, Vietnam

2 Institute of Nutrition, Mahidol University, Chang Wat Nakhon Pathom 73170, Thailand

Iodine deficiency (ID) is affecting $~ 30 \%$ of the world's population. During pregnancy, iodine requirement is high to ensure fetus development, but data on ID are limited. This study aimed to assess iodine status and sources of iodine among Vietnamese pregnant women living in a coastal region. This was cross-sectional study, whose sample population was 645 pregnant women randomly selected, stratified by topographical areas. Urinary iodine concentration (UIC) was measured in casual urine samples in order to assessed iodine status in the population, while data on demographic data were collected using a questionnaire. Household $(\mathrm{HH})$ salt samples and food frequency questionnaire (FFQ) were collected to estimate iodine intakes. Median UIC was $163.75 \mathrm{~g} / \mathrm{L}$, indicating sufficient iodine among pregnant women in the region; $43.4 \%$ had UIC $<150 \mathrm{~g} / \mathrm{L}: 4.8 \%$ severe, $15 \%$ moderate, and $23.6 \%$ mild ID. Median UIC in all four topographical areas showed sufficient iodine, but city women had significantly lower UIC than coastal and flat areas $(p<0.01)$. Ninety three percent HHs used iodized salt, and $74.4 \%$ of $\mathrm{HH}$ salt contained adequate iodine. Median UIC was significantly associated with iodized salt use and seafood consumption. Those who consumed adequately iodized salt and medium seafood could reduce $55-70 \%$ risk of ID, while those who used seasoning powder for cooking would have a $40 \%$ increase risk of ID during pregnancy. Pregnant women in the south central coastal region had sufficient iodine intake through iodized salt as an important source. Quality of salt iodization needs to be ensured at the factory and HHs level. Key words: iodine deficiency/pregnant women/iodized salt/seafood intake/urinary iodine concentration. 


\section{K. Dienelt, K. Mehta, C. Moores and J. Miller}

Nutrition and Dietetics, College of Nursing and Health Sciences, Flinders University, Australia

Sufficient information and support of breastfeeding mothers is vital to avoid feelings of being overwhelmed and encourage optimal infant feeding (IF) practices for healthy infant development. IF apps contain information and instructions around breastfeeding; however, little is known about their use or mothers attitudes towards these IF apps. This study aimed to investigate mothers use and experiences of IF apps, including benefits of app use, the apps role within IF, and how information within these apps is used. In-depth interviews $(45-60 \mathrm{~min})$ were conducted with breastfeeding mothers $(n=9)$ who had used an IF app in the last year. Interviews were digitally recorded, transcribed verbatim, and manually coded prior to thematic analysis. Coding revealed four key themes: (1) societal and generational shift towards apps for self-management, (2) meeting parenting ideals and expectations, (3) negative aspects to app use, and (4) informing decision-making. Mothers interpreted information within IF apps to inform decisions, such as whether to seek professional help, in consultation with other sources. Most mothers described the experience of IF app use as positive, but some acknowledged distrust and/or over reliance on IF apps. For some, IF app use was reassuring and increased breastfeeding confidence. Many mothers found IF apps provided feelings of control and support. Apps were relied on for tracking data, which were commonly interpreted to inform breastfeeding in conjunction with additional information sources. Future research is needed to understand the influence of app use on compliance to best practice IF guidelines.

\subsection{Associations with Diet and Daytime Sleepiness in Australian Children Aged 8-12 Years}

\section{E. J. Watson ${ }^{1}$, S. Banks ${ }^{1}$, M. Kohler ${ }^{1}$ and A. M. Coates ${ }^{2}$}

1 Centre for Sleep Research, School of Psychology, Social Work and Social Policy, University of South Australia

2 Alliance for Research in Exercise, Nutrition and Activity, Sansom Institute for Health Research, School of Health Sciences, University of South Australia, Australia

The Australian Recommended Food Score (ARFS) is a diet quality score for Australian children and adolescents. This study investigated the ARFS and adherence to dietary guidelines in 8-12-year old children and determined associations with daytime sleepiness. A cross-sectional study of 290 South Australian children aged 8-12 years (without clinically diagnosed sleep, or behavioural or dietary problems) completed the Australian Child and Adolescent Eating Survey-FFQ (ARFS was derived, maximum score: 58; higher score reflects better diet quality), and the Paediatric Daytime Sleepiness Scale (PDSS; higher score indicates greater daytime sleepiness). Based on age- and sexspecific nutrient reference values (NRVs), the majority (72-100\%) of male and female children met the recommended amount for protein, riboflavin, zinc, and vitamin- $\mathrm{C}$, while fewer children (20.0$87.5 \%$ ) met the recommended amount for fibre, calcium, potassium, and iron, and very few children met the recommended intake for sodium $(0-10 \%)$. The ARFS for the whole sample was 27.0 (17) (median (IQR); males 25.5 (17), range: 3-48; females: 28 (16), range: 5-56). The average PDSS score for the sample was $10.6 \pm 5.6$ (mean \pm SD, males: $10.4 \pm 5.8$; females: $10.8 \pm 5.8$ ). A higher food quality score was associated with less daytime sleepiness $(r=-0.130, p=0.028)$; when sexes were considered separately, this relationship only stayed significant in females $(r=-0.201, p=0.015)$. These results show that nutrient intakes of sodium, fibre, calcium, potassium, and iron are key areas of concern in this sample of children. Diet quality is an important factor to consider when assessing daytime sleepiness in children. 


\title{
2.81. Young Healthy Asian Australians Experience Elevated Postprandial Insulin Responses
}

\author{
M. Murray ${ }^{1}$, A. L. Dordevic ${ }^{1}$, L. Ryan ${ }^{2}$, M. P. Bonham ${ }^{1}$
}

1 Department of Nutrition, Dietetics and Food, Monash University, Clayton, VIC 3168, Australia

2 Department of Natural Sciences, Galway-Mayo Institute of Technology, Galway, Ireland

People of Asian descent living in Western countries experience an increased risk of type 2 diabetes. This research investigated postprandial blood glucose and insulin responses in healthy individuals of Asian and Caucasian heritage. Thirty-eight adults (12 Asian, 26 Caucasian) with normal fasting blood glucose $(<5.5 \mathrm{mmol} / \mathrm{L})$ were recruited. Fasting and postprandial capillary blood samples were taken over a two hour period following consumption of white bread ( $50 \mathrm{~g}$ available carbohydrate) to measure blood glucose and plasma insulin. Differences between ethnic groups were assessed using independent $t$-tests. There were no differences for blood glucose incremental area under the curve (iAUC) or peak concentration $(\mathrm{mmol} / \mathrm{L})$. However, Asian participants demonstrated increased iAUC (Asian: 3489 (4061) U/mL·min; Caucasian: 2545 (2472) U/mL·min) and peak concentration (Asian: 67.7 (91.8) U/mL; Caucasian: 46.3 (40.3) U/mL) for plasma insulin, compared with those of a Caucasian background, $p=0.022$ and 0.010 , respectively. In a young, healthy population, participants who identified as being of Asian descent had an elevated postprandial insulin response compared with their Caucasian counterparts. These results indicate reduced insulin sensitivity in Asian people, and that glucose tolerance tests alone may be insufficient to diagnose diabetes risk in Asian populations.

\subsection{The Effects of Intermittent Compared to Continuous Energy Restriction on Glycaemic Control in Type 2 Diabetes}

\section{S. Carter, P. M. Clifton and J. B. Keogh}

School of Pharmacy and Medical Sciences, University of South Australia, Sansom Institute for Health Research. Alliance for Research in Exercise, Nutrition and Activity (ARENA), Adelaide 5001, Australia

Our aim was to investigate the effects of intermittent (IER) compared to continuous energy restriction (CER) on glycaemic control in type 2 diabetes mellitus (T2DM), with weight loss as a secondary aim. Ninety-one (61 \pm 8 years) participants (BMI $\left.35.7 \pm 5.9 \mathrm{~kg} / \mathrm{m}^{2}\right)$ with T2DM $(\mathrm{HbA} 1 \mathrm{c} 7.1$ $\pm 1.2 \%)$ were randomised to a 2-day very-low-energy diet $(2100-2500 \mathrm{~kJ})$ with 5 days of habitual eating or a CER diet (5000-6300 kJ/day) for 52 weeks. Participants were seen fortnightly for 12 weeks and then every 8 weeks for 40 weeks. Medications were modified at baseline according to baseline $\mathrm{HbA1c}$ (IER) and in response to reported hyperglycaemia $(>10 \mathrm{mmol} / \mathrm{L})$ or hypoglycaemia $(<4$ $\mathrm{mmol} / \mathrm{L})$ and weight loss in both groups. In 63 participants, HbA1c decreased $(p<0.001)$ with no difference between groups at $12(-0.7 \pm 0.9 \%$ CER, $-0.5 \pm 0.7 \%$ IER; $p=0.4)$ or at 52 weeks $(-0.5 \pm 1.0 \%$ CER, $-0.3 \pm 0.6 \%$ IER; $p=0.2)$. Baseline HbA1c (7.3\% CER vs. $6.8 \%$ IER) predicted HbA1c change $(\mathrm{r}=$ $-0.7 ; p<0.001)$ and after adjusting for baseline difference, $\mathrm{HbA1c}$ calculated change was similar for both groups $(-0.4 \% ; p=0.7)$ at 52 weeks. Medications decreased at 12 weeks $(-0.4 \pm 0.9 ; p=0.002)$ and at 52 weeks $(-0.5 \pm 1.0 ; p=0.002)$. Weight decreased $(p<0.001)$ with no difference between groups at $12(-5.6 \pm 4.2 \mathrm{~kg}$ CER, $-6.2 \pm 3.6 \mathrm{~kg}$ IER; $p=0.5)$ or at 52 weeks $(-5.1 \pm 5.9 \mathrm{~kg}$ CER, $-5.7 \pm 5.6 \mathrm{~kg}$ IER; $p$ $=0.7)$. All measures of body composition decreased over time $(p<0.001)$. A 2-day IER improves glycaemic control, weight loss, and medications similar to CER.

\subsection{Fish Intake and Incidence of Type 2 Diabetes: A Prospective Analysis of the AusDiab Cohort}

A. J. Owen ${ }^{1}$, A. Ridhanya ${ }^{1}$, D. Liew ${ }^{1}$, J. Shaw ${ }^{2}$ and D. J. Magliano ${ }^{1,2}$

1 School of Public Health \& Preventive Medicine, Monash University, Melbourne, Australia

2 Baker Heart and Diabetes Institute, Melbourne, Australia

(1) Fish intake has been found to be associated with diabetes risk, but there is some evidence of geographic variation in this association, with northern hemisphere cohorts finding a positive association between fish/omega-3 intake and diabetes incidence, while a number of Asian cohorts 
have found fish intake is associated with reduced risk of diabetes. This study examined the relationship between fish intake and diabetes risk in the Australian Diabetes, Obesity, and Lifestyle (AusDiab) cohort, a national population-based cohort study from Australia. (2) In the AusDiab cohort, 5817 adult participants had complete baseline and follow-up data available, among whom 434 developed diabetes (measured by oral glucose tolerance test) over follow-up. Fish intake was measured using a validated semi-quantitative food frequency questionnaire. Cox-regression was used to examine the relationship between types of fish intake and diabetes risk. (3) Consuming tinned fish frequently (two or more serves per week) was associated with a higher risk of diabetes (HR 1.4, 95\% CI 1.04-1.88). Occasional fried fish consumption was also positively associated with diabetes (HR 1.31, 95\% CI 1.02-1.68). In minimally-adjusted models (by age and sex), intake of non-fried fish and total fish was inversely related with diabetes risk, but this was lost in fully-adjusted models. (4) This study found weak evidence that moderate intake of total fish and non-fried fish intake is associated with reduced risk of diabetes. However, consuming fish frequently or consumption of fried fish negates the protective effect, and may increase risk.

\subsection{The Effect of Meal Timing on Postprandial Glucose and Insulin Response: A Meta-Analysis of Acute Experimental Trials}

G. K. W. Leung ${ }^{1}$, C. E. Huggins ${ }^{1}$, R. Ware ${ }^{2}$ and M. B. Bonham ${ }^{1}$

1 Department of Nutrition, Dietetics and Food, Monash University, Australia

2 Menzies Health Institute Queensland, Griffith University, Australia

Eating at night has been linked to poor postprandial glycaemic control and may be a risk factor for development of metabolic conditions. This systematic review aimed to synthesise the literature to determine the effect of eating at night on postprandial glucose and insulin responses. The PRISMA guidelines were followed. Seven databases were searched (April 2016). Inclusion criteria were identical test meals administered during the day $(0700 \mathrm{~h}-1600 \mathrm{~h})$ and night $(2000 \mathrm{~h}-0400 \mathrm{~h})$ to the same participants, after a minimum fast of three hours. Meta-analyses with fixed-effects models and an estimated correlation coefficient of 0.4 for glucose and 0.3 for insulin were undertaken using STATA. The primary analysis included studies with day test meals provided before midday, and the secondary analysis included day test meals provided after midday. Thirteen studies met the inclusion criteria. Eight studies had data for meta-analysis, of which seven studies (10 comparisons) were included in the primary analysis for glucose and six studies (nine comparisons) for insulin. Postprandial glucose was lower during the day compared with night (standardised mean difference (SMD) $-1.84 ; 95 \%$ confidence interval (CI): $-2.22,-1.45 ; \mathrm{I}^{2}=35.8 \%$ ). Postprandial insulin was not consistently different between day and night (SMD $-0.19 ; 95 \% \mathrm{CI}:-0.52,0.15 ; \mathrm{I}^{2}=14.5 \%$ ). The secondary meta-analyses of glucose ( $n=5$ comparisons) and insulin ( $n=4$ comparisons) showed no significant differences between day and night. Overall, postprandial glucose response is higher at night compared with the morning. Habitual night time food intake may be associated with increased risk of type-2 diabetes and cardiovascular disease through elevated postprandial glucose.

\subsection{Perilla Oil Supplementation Improves Hypertriglyceridemia and Gut Dysbiosis in Diabetic KKAy Mice}

\section{F. Wang, M. Hu, H. Zhu, L. Yang and G. Sun}

Key Laboratory of Environmental Medicine and Engineering of Ministry of Education, and Department of Nutrition and Food Hygiene, School of Public Health, Southeast University, Nanjing 210009, China

The effects of fish oil-derived $\omega-3$ polyunsaturated fatty acids (PUFA) on diabetes have been investigated intensively, but our knowledge about the anti-diabetic effects of perilla oil-derived $\omega-3$ PUFA are limited. The aim of this study was to examine whether perilla oil supplementation would improve glucolipid metabolism and modulate gut microbiota in diabetic KKAy mice. The successfully established diabetic KKAy mice were randomized into 4 groups: (1) diabetic model (DM), (2) low-dose perilla oil (LPO, $670 \mathrm{mg} / \mathrm{kg}$ bw/day), (3) middle-dose perilla oil (MPO, $1330 \mathrm{mg} / \mathrm{kg}$ bw/day), or (4) high-dose perilla oil (HPO, $2000 \mathrm{mg} / \mathrm{kg}$ bw/day). C57BL/6J mice were fed a chow diet 
as a normal control (NC) group. At the end of 12 weeks, mice were euthanized and glucolipid indications were analyzed. Histological studies were performed using HE staining. Bacterial genome DNA was isolated from mice' colonic faeces. Gut microbiota analysis were investigated based on the sequencing results on V4 region of $16 \mathrm{~S}$ rRNA. Although serum glucose, insulin, total cholesterol, low density lipoprotein cholesterol, and high density lipoprotein cholesterol were unchanged, serum triglyceride significantly decreased in LPO, MPO, and HPO compared with DM. The histopathological changes of hepatocellular macrovesicular steatosis and adipocyte hypertrophy were ameliorated by perilla oil supplementation, especially in HPO. A principal component analysis showed NC and HPO were clearly separated from DM, LPO, and MPO, while DM, LPO, and MPO were relatively coherent. These findings indicate that perilla oil-derived $\omega-3$ PUFA could be used to prevent hypertriglyceridemia and gut dysbiosis in diabetic individuals.

\subsection{Dietary Pattern of Type 2 Diabetic Patients in Northern Nigeria}

\section{S. M. Abubakar*, H. Abubakar, B. G. Kurfi and S. A. Garba}

Department of Biochemistry, Bayero University, P. M. B., Kano 3011, Nigeria

* Correspondence: smabubakar.bch@buk.edu.ng

Compliance to dietary advice by type 2 diabetic patients has been an issue of concern in disease management in northern Nigeria. Diabetic complications are in part associated with unhealthy dietary habits of patients on medication. This study assessed the dietary habits of diabetic patients in northern Nigeria to ascertain the degree of compliance with dietary advice. Seventy-two (72) type 2 diabetic patients attending diabetic clinic of Murtala Muhammad Specialist Hospital Kano volunteered and participated in the study. Structured questionnaire was administered to obtain basic information. Food frequency questionnaire was administered on the 1st, 7th, and 14th days of the study. On each of these days, fasting blood glucose was measured to assess improvement in hyperglycaemia. Kano State Ministry of Health gave ethical approval for the conduct of this research. Results have shown poor compliance with dietary advice by the volunteers. Breakfast pattern showed that $27 \%$ of volunteers consume white bread, $33 \%$ consume millet-based pap, and $26 \%$ consume sugar-sweetened tea each 3 to 6 times in a week. In addition, 95\% of volunteers never consume fruits and/or vegetables at breakfast. The observed lunch and dinner trend is same as that of breakfast. On the other hand, mean fasting bool glucose of the volunteers remains high $(\geq 9.78$ $\mathrm{mmol} / \mathrm{L})$ and did not differ significantly $(p>0.05)$ from day 1 to 14 . The findings of this study suggests the need for persistent nutrition education and counselling in routine care for type 2 diabetic patients in northern Nigeria.

\subsection{Positive Effects of Naked Oat on Glycemia and Blood Lipid Risk Factors for Cardiovascular Disease in} Patients with T2DM

Ye Li and Yong, $\mathrm{Li}$ *

Department of Nutrition and Food Hygiene, School of Public Health, Peking University, Beijing, China * Corresponding Author

(1) Background: Patients with T2DM and metabolic syndrome are at increased risk for cardiovascular complications. The aim of this study was to evaluate the effects of long-term naked oat consumption on cardiovascular disease risks in patients with T2DM and metabolic syndrome. (2) Methods: 244 participants were selected from a randomized clinical trial who completed the one year dietary program. They were randomly allocated to four groups: control, diet, $50 \mathrm{~g}-\mathrm{NOG}$, or $100 \mathrm{~g}$ NOG group. The physical examinations and blood tests were carried out, and food-frequency questionnaires were administered to investigate changes from baseline and compliance with the dietary intervention. (3) Results: After 1 year, HbA1c, FPG, and PG were significantly decreased compared to the control group particularly. Reductions were also achieved in TG, TC, and LDL-c in the intervention groups $(p<0.05)$. The groups differed significantly with respect to the percent of patients who reached the treatment goals in glycosylated hemoglobin, cholesterol, and triglycerides 
$(p<0.05)$. Glycemic control was better in patients of 100 g-NOG group with $23.8 \%$ having HbA1c below $6.5 \%$. (4) Conclusions: The results of the present study suggest the beneficial effects of naked oats on cardiovascular disease risk factors.

\subsection{Dietary Carbohydrate Quality Is Associated with Total and LDL Cholesterol among Young Malaysian Indians}

Yeng Yi Ng ${ }^{1}$, Juen Jiat Lee ${ }^{1}$, Sangeetha Shyam ${ }^{1}$, Ramachandran Valsala ${ }^{2}$ and Misra Snigdha ${ }^{1}$

1 Department of Nutrition \& Dietetics, International Medical University, Jalan Jalil Perkasa 19, Bukit Jalil, 57000 Kuala Lumpur, Malaysia

2 Department of Applied Biomedical Sciences and Biotechnology, International Medical University, Jalan Jalil Perkasa 19, Bukit Jalil, 57000 Kuala Lumpur, Malaysia

Evidence shows that risk of cardiovascular diseases (CVD) increases with the consumption of high glycaemic index (GI) and glycaemic load (GL) carbohydrates, especially among populations with insulin resistance. Indian ethnicity has a greater propensity for insulin resistance, which is yet to be established. Hence, this cross-sectional study investigates the association of carbohydrate quality with fasting serum lipids among selected young healthy Malaysian Indians. Eighty subjects (21 men, 59 women) were recruited. Dietary intake was recorded using a food frequency questionnaire and analysed using DietPLUS version 3. Serum lipids were measured using a Cardiochek PA. Average dietary GI and GL were 57 and 164 units, respectively. Despite their young age (median 22 (4) years) and acceptable BMI range $\left(22.3 \pm 6.8 \mathrm{~kg} / \mathrm{m}^{2}\right), 69 \%$ had LDL-cholesterol $>2.59$ $\mathrm{mmol} / \mathrm{L}, 46 \%$ had TC: HDL ratio $>3.5 \mathrm{mmol} / \mathrm{L}$, and $4 \%$ had triglycerides $>1.69 \mathrm{mmol} / \mathrm{L}$. GI, and LDLcholesterol was significantly correlated $(\mathrm{r}=0.261, p=0.019)$. After adjustment for caloric intake, GL was correlated with total $(r=0.258, p=0.023)$ and LDL-cholesterol $(r=0.268, p=0.016)$. Stepwise regression model showed that every 10 unit decrease in GL/1000 kcal resulted in $0.2 \mathrm{mmol} / \mathrm{L}$ decrease in total cholesterol. Furthermore, every 10 unit decrease in GI also decreased LDL by $0.54 \mathrm{mmol} / \mathrm{L}$ $(\sim 10 \%$ CVD risk-reduction). Thus, diets high in GI and GL significantly increase the serum lipid profile among this group. This suggests an increased consumption of whole grains in the diet may reduce the risk of CVD.

\subsection{Beneficial Effects of Astaxanthin on Glycemic Control and Lipid Profile in Healthy Volunteers}

M. Urakaze, C. Kobashi, Y. Satou, K. Shigeta, M. Toshima, K. Yamakoshi, M. Takagi, J. Takahashi and M. Nishida

Anti-Aging Medical Research Center, Faculty of Life and Medical Sciences, Doshisha University, Japan

Astaxanthin is found in varying concentrations in unicellular microalgae, plankton, krill and other seafood such as salmon, trout, and crustaceans, including crayfish. Astaxanthin is a potent quencher of free radicals and reactive oxygen and nitrogen species. It is 10 times more potent as a singlet oxygen quencher than beta carotene, and 100 times greater than alpha tocopherol. Both its high potency and polar properties make astaxanthin an attractive nutraceutical for further investigation in diabetes, lipid profile, and atherosclerotic cardiovascular disease in which antioxidant cellular protection may be of clinical benefit. Several clinical studies have reported that astaxanthin has favorable effects on glycemic control and lipid profile. Here, we report that clinical study of astaxanthin (randomized, double-blind, placebo-controlled study) on glucose metabolism and lipid profile in healthy volunteers. After astaxanthin (12 mg/day) supplementation for 12 weeks, the glucose level at $120 \mathrm{~min}$ by $75 \mathrm{~g}$ OGTT was significantly decreased compared to before. Besides, the levels of $\mathrm{HbA1c}(5.64 \pm 0.33$ to $5.57 \pm 0.39, p<0.05)$, apo $\mathrm{E} 4.43 \pm 1.29 \mathrm{mg} / \mathrm{dL}$, to $4.13 \pm 1.24 \mathrm{mg} / \mathrm{dL}$, $p<0.05$, and MDA-LDL87.3 $\pm 28.6 \mathrm{U} / \mathrm{L}$ to $76.3 \pm 24.6 \mathrm{U} / \mathrm{L}, p<0.05$ were also reduced, although none of TC, TG, and HDL-C was changed. Our results suggest that astaxanthin might have beneficial effects on the prevention of diabetes and atherosclerosis. 


\title{
2.90. Glycaemic Index Testing of Standard and High Sugar Rodent Diets In Vivo in C57BL/6 Mice
}

\author{
G. J. Campbell ${ }^{1}$, D. P. Belobrajdic ${ }^{2}$ and K. S. Bell-Anderson ${ }^{1}$
}

1 University of Sydney, Charles Perkins Centre, School of Life and Environmental Sciences, Australia

2 Commonwealth Scientific and Industrial Research Organization (CSIRO) Health and Biosecurity, Adelaide 5000, Australia

The glycaemic index (GI) is a useful tool that enables the glycaemic reponse of foods to be compared in humans, but one limitation is its difficulty in assessing the glycaemic impact of different sugars and starches independent of other cofounding factors such as dietary fibre, fat, and protein, in a controlled diet context. To address this, the aim of this study was to use mice to assess the GI of nine high-carbohydrate diets that varied in sugar/starch source, yet were isocaloric, with similar macronutrient and fibre composition. Following the standard for GI testing in humans in a crossover design, the GI of the following diets were tested in ten mice: glucose control, Cooked Cornstarch (CC), Raw Cornstarch (RC), chow, High Maltodextrin (HM), High Glucose (HG), High Sucrose (HS), High Palatinose (HP), and High Fructose (HF). All eight meals were significantly different from the glucose control (GI: 100; $p<0.0001)$. HG (51.89 \pm 3.44$)$, HM ( $51.8 \pm 6.27)$, CC (50.01 \pm 4.15$),$ RC (49.84 $\pm 6.28)$, and chow $(43.92 \pm 3.77)$ were not significantly different from each another. HS $(30.79 \pm 3.83)$, $\mathrm{HP}(23.78 \pm 4.51)$, and HF $(17.77 \pm 2.02)$ were significantly different from the other diets $(p<0.05)$. This is the first trial to report GI testing in mice, resulting in four main findings: chow was high GI; digestibility of raw and cooked cornstarch was similar; the GI of different sugars occur in the same order as that reported in humans; and, lastly, we confirm the utility of the standard GI human protocol adapted to mice.

\subsection{Vitamin D Status and Infant Body Composition and Skinfold Thickness at Birth}

P. Dwarkanath, T. Thomas, S. Mehta, S. Joseph, A. Thomas and A. Kurpad

1 Division of Nutrition, St. John's Research Institute, Bangalore, India

2 Department of Biostatistics, St. John's Medical College \& Research Institute, Bangalore, India

3 Division of Nutritional Sciences, Cornell University, Ithaca, New York, United States

4 Agilent Technologies, Market Development Center, Bangalore, India

5 Department of Obstetrics and gynecology, St. John's Medical College Hospital, Bangalore, India

6 Department of Physiology, Division of Nutrition, St. John's Medical College \& Research Institute, Bangalore, India

High prevalence of vitamin-D deficiency exists in pregnant women in India ( 90\%). Evidence suggests a pivotal role of vitamin-D during pregnancy in maternal and neonatal outcomes. Prenatal vitamin-D deficiency is detrimentally associated with pregnancy-related morbidities, birthweight, rickets, and adiposity in infants. We examined the association between 1 st trimester vitamin-D status and levels in cord-blood with neonatal body-composition and skinfold thicknesses at birth.

Apparent healthy pregnant women $(n=419)$ were recruited $\sim 12$ weeks of gestation and followed-up until delivery. Baseline information (socio-economic-status, obstetric history) at recruitment, dietary intakes, anthropometry, and morbidity details were collected during pregnancy. At delivery, birth outcomes recorded, baby anthropometry performed and body-composition parameters (sum of skinfold-thickness, arm-muscle-area), and central to skinfold-ratio were computed using standard equations. Vitamin-D $(25(\mathrm{OH}) \mathrm{D})$ concentrations were assessed using tandem liquid chromatography-mass-spectrophotometry (LC-MS/MS) in 1st trimester and in cordblood at delivery. Linear regression model was used to evaluate the association between infant bodycomposition and skinfold thickness with $(25(\mathrm{OH}) \mathrm{D})$ in mother and cord-blood.

Eight-two percent of mothers at recruitment and infants at birth (cord-blood) were $25(\mathrm{OH}) \mathrm{D}$ deficient $(<50.0 \mathrm{nmol} / \mathrm{L})$. Positive correlation was observed between $25(\mathrm{OH}) \mathrm{D}$ status in 1 st trimester and cord-blood $(\mathrm{r}=0.426, p=0.000)$. No significant correlation was observed between neonatal bodycomposition parameters and 25(OH)D levels. Multilinear regression analysis showed a significant negative association between the cord-blood $25(\mathrm{OH}) \mathrm{D}$ and the 'central to peripheral' skinfold-ratio at birth $(\beta=-0.17, p=0.021)$, after adjusting for potential confounders. 
High prevalence of vitamin-D exists during pregnancy and in newborns. Lower vitamin-D levels in cord-blood may be indicative of increased skinfold thickness-ratio, a marker of 'fatness' in neonates.

\title{
2.92. Acute Changes in Plasma Zinc Concentration Following a Bout of Maximal Exercise Testing -Pilot Analysis of a RCT
}

A. Chu ${ }^{1}$, T. Varma ${ }^{1}$, M. Reid ${ }^{2}$, P. Petocz ${ }^{3}$ and S. Samman ${ }^{1,4}$

1 Department of Human Nutrition, University of Otago, Dunedin 9016, New Zealand

2 Department of Chemistry, University of Otago, Dunedin 9016, New Zealand

3 Department of Statistics, Macquarie University, Sydney NSW 2000, Australia

4 School of Life and Environmental Sciences, University of Sydney, Sydney NWS 2000, Australia

Our previous meta-analyses showed that although exercise induces changes in serum zinc concentrations; the quantified results may be confounded by not adjusting for exercise-induced changes in blood volume. The present trial aims to determine the effect of an exercise bout on zinc biomarkers in healthy adults. Men and women were recruited to participate in a randomised, crossover, time-course trial. All participants completed an exercise and a control session in random order; blood samples were collected at baseline, immediately, $30 \mathrm{~min}, 1 \mathrm{~h}$, and $2 \mathrm{~h}$ after exercise/control period. In the exercise session, participants completed a $\mathrm{VO}_{2 \max }$ test on the cycle ergometer. Plasma zinc concentration, haematocrit, zinc transporters, and metallothioneins gene expressions were measured. Changes in plasma volume (\%) were calculated. Repeated measures ANOVA models were used to determine paired differences in the time course periods of all outcomes. In an initial analysis of 14 participants, plasma zinc concentration profiles were significantly different between the exercise and control sessions ( $p$-interaction $<0.001)$. In the exercise session, plasma zinc concentrations significantly increased by $14.7 \pm 2.6 \%$ from baseline (mean \pm SEM) immediately following exercise, while plasma volume decreased by $13.1 \pm 1.6 \%$. When plasma zinc concentrations were adjusted for changes in plasma volume, no statistically significant changes in plasma zinc were noted. This pilot analysis indicates that significant acute changes in plasma zinc concentrations occur following exercise; however, the fluctuations are negated when exercise-induced changes in plasma volume are considered.

\subsection{Disparities Exist between Dietary Intake of Indigenous Australian Pregnant Women and Australian Dietary Guidelines}

\author{
Y. Q. Lee ${ }^{1,2}$, C. E. Collins ${ }^{1,3}$, A. Gordon ${ }^{4}$, K. M. Rae ${ }^{1,2}$ and K. G. Pringle ${ }^{1,2}$ \\ 1 Faculty of Health and Medicine, University of Newcastle, Australia \\ 2 Priority Research Centre for Reproductive Sciences, University of Newcastle, Australia \\ 3 Priority Research Centre in Physical Activity and Nutrition, University of Newcastle, Australia \\ 4 Charles Perkins Centre, University of Sydney, Australia
}

Optimal nutrition during pregnancy is critical for fetal growth, development, and long-term health outcomes. Little is known about the adequacy of macro- and micronutrient intakes and overall diet quality of Indigenous Australian pregnant women. The aim was to assess nutrient sufficiency and diet quality, measured using the Australian Recommended Food Score (ARFS), in a cohort from the Gomeroi Gaaynggal Study ( $\mathrm{n}=62)$. Dietary intake was assessed using the Australian Eating Survey (AES) food frequency questionnaire (FFQ), which was self-administered during the 3rd Trimester ( $\geq 27$ weeks). Food group servings and nutrient intakes were compared to the Australian Guide to Health Eating (AGHE) and Australian Nutrient Reference Values (NRVs). No women met all AGHE daily food group serving recommendations. The highest adherence rates were for (proportion, median (IQR) servings/day), meat/alternatives (32\%, 2.6 (1.7-3.8)), vegetables (31\%, $3.4(2.2-5.2))$, and fruit $(29 \%, 1.3(0.46-2.2))$ groups. Almost $92 \%$ exceeded the recommended intakes of energy-dense, nutrient-poor foods, and \% energy from saturated fat was excessive $(15 \%)$. A small percentage of pregnant women achieved the NRVs from dietary sources alone, including folate (6.5\%), iron (9.7\%), and fibre (27.4\%). Only 6.5\% achieved all NRVs (folate, iron, calcium, zinc, and fibre) important in pregnancy. The median ARFS was 28 points (max 73). Dietary intakes of Indigenous Australian 
pregnant women do not align with the AGHE nor meet many pregnancy-specific NRVs. Strategies to optimise nutrient intakes of Indigenous pregnant women are urgently needed.

\title{
2.94. Sodium Intake Was Positively Associated with Cortisol Excretion in a Cross-Sectional Sample of Australian School Children
}

\author{
S. J. Torres ${ }^{1}$, C. Grimes ${ }^{1}$, C. A. Nowson ${ }^{1}$, S. U. Jayasinghe ${ }^{1,2}$, C. Bruce ${ }^{1}$, S. Mason ${ }^{1}$, F. He ${ }^{3}$ and A. I. \\ Turner $^{1}$ \\ 1 Institute for Physical Activity and Nutrition, School of Exercise and Nutrition Sciences, Deakin University, \\ Victoria, Australia \\ 2 Biomedical Sciences, Cornell University, Ithaca, NY, USA \\ 3 Centre for Environmental and Preventative Medicine, Wolfson Institute of Preventative Medicine, Queen \\ Mary University of London, London EC1M 6BQ, UK
}

The detrimental effects to health of high sodium intake and chronically elevated cortisol levels are well known. There is also evidence in adults that high sodium intake is associated with high levels of urinary cortisol excretion. We aimed to assess, in mothers and children, if urinary excretion of sodium and potassium was associated with urinary excretion of cortisol and cortisol metabolites. A cross-sectional sample of school children and their mothers performed $24 \mathrm{~h}$ urine collection in which sodium, potassium (ion selective electrode), free cortisol (enzyme-linked immunosorbent assay), and cortisol metabolites (gas chromatography-mass spectrometry) were measured. School children ( $\mathrm{n}=$ 120 ; age $9.2 \pm 2.0$ years mean $( \pm S D)$ ) and their mothers $(n=102 ; 41.7 \pm 5.1$ years $)$ participated. Sodium and potassium excretion was $104.2 \pm 61.8$ and $46.8 \pm 18.5 \mathrm{mmol} / 24 \mathrm{~h}$, respectively, in children and $120.0 \pm 41.3$ and $67.6 \pm 18.5 \mathrm{mmol} / 24 \mathrm{~h}$, respectively, in mothers. Multiple regression analysis adjusting for age, sex, and body mass index z score indicated in children, not mothers, that sodium excretion was positively associated with urinary free cortisol $\left(\beta=0.38(0.13,0.61 ; 95 \% \mathrm{CI}), \mathrm{R}^{2}=0.41, p<0.001\right)$ and urinary cortisol metabolites $\left(\beta=0.009(0.004,0.013), \mathrm{R}^{2}=0.35, p<0.001\right)$. Positive associations were also observed between potassium excretion and urinary free cortisol $\left(\beta=1.08(0.53,1.62), R^{2}=\right.$ $0.34, p<0.001)$ and urinary cortisol metabolites $\left(\beta=0.03(0.01,0.04), \mathrm{R}^{2}=0.33, p<0.001\right)$ in children only. Population salt reduction programs may have additional benefits with regard to reducing cortisol levels in children.

\subsection{The Effects of Zinc Supplementation on Copper Status: A Systematic Review and Meta-Analysis}

\author{
M. Foster ${ }^{1}$, A. Chu ${ }^{1}$, V. P. C. Tan ${ }^{1}$, T. Varma ${ }^{1}$, P. Petocz ${ }^{2}$ and S. Samman ${ }^{1,3}$ \\ 1 Department of Human Nutrition, University of Otago, Dunedin 9054, New Zealand \\ 2 Department of Statistics, Macquarie University, NSW 2109, Australia \\ 3 School of Life and Environmental Sciences, University of Sydney, NSW 2006, Australia
}

Recent studies suggest zinc supplementation is beneficial for ameliorating chronic disease risk. In contrast, some early studies report adverse effects of excess zinc intake on copper metabolism. The present systematic review and meta-analysis investigate the effects of zinc supplementation on copper status in humans. A literature search was conducted of Embase, Scopus, CENTRAL, and MEDLINE databases from inception to 20 February 2017. Twenty-nine randomized controlled trials that examined the effects of zinc supplementation on measures of copper status qualified for inclusion in the systematic review. Sufficient data were available to conduct meta-analyses in healthy populations of the effects of zinc supplementation on serum copper concentrations and erythrocyte superoxide dismutase (E-SOD) activity. Supplementation with $25-30 \mathrm{mg}$ zinc/d for 3-6 months (5 interventions) resulted in a decrease in serum copper concentrations of $0.80 \mu \mathrm{mol} / \mathrm{L}$ (95\% CI: -1.44 , $-0.16 ; p=0.01$ ); however, upon removal of one large study in sensitivity analyses, this finding was no longer significant. Zinc supplementation of $30-60 \mathrm{mg} / \mathrm{d}$ for 6 weeks- 6 months (6 interventions) showed no effect on E-SOD activity. In a qualitative synthesis of populations with chronic disease, the balance of evidence found no adverse effects of zinc supplementation on copper status. Supplementation with zinc over a prolonged period does not appear to affect markers of copper 
status in humans. Revision of the upper level (UL) of zinc intake is warranted, given that the UL is based on the assumption that zinc has an adverse effect on copper metabolism.

\subsection{Adequate Dietary Iodine Intake of Australian Population Dependent on Bread Consumption: Data Post-Mandatory Fortification}

\section{K. E. Charlton, Y. C. Probst and G. Kiene}

School of Medicine, Faculty of Science, Medicine and Health, University of Wollongong, Australia

To address mild iodine deficiency in Australia, a mandatory fortification program of iodised salt in bread was implemented in 2009. This study aimed to determine factors associated with achieving an adequate dietary iodine intake in the population post-fortification, and to assess whether bread consumption patterns affect iodine intake in high-risk groups. Using nationally representative data of repeated 24-h recalls from the 2011-2012 Australian Health Survey, dietary iodine intakes and food group contributions were compared by age, socioeconomic status (SES), and geographical remoteness $(\mathrm{N}=7735)$. Association between fortified bread intake and adequacy of iodine intake was investigated using logistic regression models in women aged $14-50$ y $(n=3496)$ and children aged 2$18 \mathrm{y}(\mathrm{n}=1772)$. Main sources of iodine intake at the time of the survey were cereal and cereal products, followed by milk products and dishes. Differences in iodine intake and dietary iodine habits according to age, SES, and location were found $(p<0.001)$ for women of child-bearing age. Fortified bread consumption at $\geq 100 \mathrm{~g} /$ day was associated with five times greater odds of achieving an adequate iodine intake (OR 5.0, 95\% CI 4.96-5.13; $p<0.001$ ) compared to lower bread consumption in women and 12 times in children (OR 12.34, 95\% CI 1.71-89.26; $p<0.001$ ). Disparities in dietary iodine intake exist within sectors of the population, even after mandatory fortification of a staple food. On-going monitoring and surveillance of iodine status is required, and other vehicles for fortification may be required if bread consumption patterns decline.

\subsection{The Effect of Advanced Glycation End-Products on Bone-Quality Deterioration and Possible Prevention with Tocotrienol}

A. Ishitsuka ${ }^{1}$, K. Kono ${ }^{2}$, W. Yamada ${ }^{1}$, N. Miyanishi ${ }^{1,2}$ and T. Yano ${ }^{1,2}$

1 Graduate School of Food and Nutrition Science, Toyo University, Itakura, Japan

2 Research Institute of Life Innovation, Toyo University, Tokyo, Japan

Reduction of bone mineral density and degradation of bone quality are causes of osteoporosis. Bone quality is defined by enzymatic crosslink and non-enzymatic cross-link. Enzymatic crosslink is formed by lysyloxidase (LOX) and makes bone flexible. Recently, it has been suggested that advanced glycation end-products (AGEs) that are non-enzymatic cross-link decreased LOX expression, but this mechanism is not been clear. Meanwhile, tocotrienol (T3), vitamin isoforms, has pharmacological activity independent of antioxidant activity. Thus, in this study, we investigated the influence of AGEs on LOX level and the preventive effect of tocotrienol on the AGEs-influenced LOX level in vitro. In this study, as T3, we used tocotrienol rich fraction (TRF) obtained from annatto. First, MG63 was grown in $0 \sim 100 \mathrm{~g} / \mathrm{mL}$ AGEs and assayed mRNA expression by pRT-real-time PCR. Next, we examined mechanism of AGEs-influenced LOX expression using siRNA. Finally, MG63 was treated with $10 \mathrm{~g} / \mathrm{mL}$ TRF and grown in $100 \mathrm{~g} / \mathrm{mL}$ AGEs; then, it was assayed mRNA expression. The result showed that LOX mRNA expression was inhibited after treatment of $100 \mathrm{~g} / \mathrm{mL}$ AGEs $(p<0.01)$, and IL6 mRNA expression was increased tendency in a dose dependent manner. LOX mRNA expression of silL6/AGEs group showed increased tendency compared with NCsiRNA, NCsiRNA/AGEs groups. LOX mRNA expression of TRF/AGEs treatment group significantly increased compared with that of AGEs treatment group $(p<0.01)$. It is suggested that AGEs influence bone quality by suppressing LOX level, and T3 can be expected as functional food component that prevents deterioration bone quality by recovering AGEs-influenced LOX level. 


\subsection{Fluoride Intakes of 9-10-Year Old Children Living in Fluoridated and Non-Fluoridated New Zealand} Cities: A Pilot Study

\section{S. Skeaff ${ }^{1}$, C. Davenport ${ }^{1}$, C. Watts ${ }^{1}$ and B. Drummond ${ }^{2}$}

1 Department of Human Nutrition, University of Otago, Dunedin 9054, New Zealand

2 Department of Oral Sciences, University of Otago, Dunedin, New Zealand

Internationally, most studies measuring fluoride intakes focus on children aged 0-8 years, because this is when permanent teeth are formed, but oral health is important across the lifespan. This study aimed to measure fluoride intakes in children aged 9-10 years living in fluoridated and non-fluoridated NZ cities. Twenty children from the cities of Dunedin $(\mathrm{n}=10$; fluoridated) and Timaru ( $\mathrm{n}=10$; non-fluoridated) were recruited in April 2017. The following data was collected from each child: one-day weighed diet record, 24-h urine sample, information about tooth brushing, and expectorated toothpaste/saliva; repeat collections were obtained from 11 children. The mean age of the children was 9.6 years, and $70 \%$ of the children were girls. Mean (SD) energy intake was 7278 (1391) kJ/day. Mean (SD) dietary fluoride intake was 0.71 (36) $\mathrm{mg} /$ day in Dunedin and 0.21 (06) $\mathrm{mg} /$ day in Timaru children. Fluoride ingested from toothpaste was 0.95 (0.41) mg/day in Dunedin and $0.99(0.52) \mathrm{mg} /$ day in Timaru children. Total fluoride intake (diet + toothpaste) for children from both cities (1.66 and $1.10 \mathrm{mg} /$ day) was below the Adequate Intake (AI) of $2.0 \mathrm{mg} /$ day. Of the total daily fluoride consumed, $23 \%$ was excreted in the urine. The results of this pilot study indicate that fluoridated water increases total fluoride intake by $\sim 30 \%$; ingested fluoride from toothpaste contributed $90 \%$ of total fluoride in children living in the non-fluoridated city of Timaru. Regardless of water fluoridation, children in this study did not meet the AI for fluoride and were well below the upper limit of $10 \mathrm{mg} /$ day.

\subsection{High Intra-Individual Variability in Dietary Flavonoid Intake Following Repeated Dietary Measures in Older Australian Adults}

\section{K. Kent ${ }^{1}$, K. Charlton ${ }^{2,3}$, J. Russell ${ }^{4}$, P. Mitchell ${ }^{5}$ and V. Flood ${ }^{6}$}

1 Faculty of Health, University of Tasmania, Tasmania, Australia

2 Faculty of Science, Medicine and Health, University of Wollongong, Wollongong, Australia

3 Illawarra Health and Medical Research Institute, Wollongong, Australia

4 Faculty of Social Sciences, University of Wollongong, Wollongong, Australia

5 Centre for Vision Research, University of Sydney, Sydney, Australia

6 Faculty of Health Sciences, University of Sydney, Sydney, Australia and Western Sydney Local Health District, Sydney, Australia

It is well established that an individual has considerable day-to-day variation in nutrient intake, which adversely effects the reliability of dietary assessment in studies requiring estimates of usual intake. However, the variability associated with dietary flavonoid intakes has not been considered. There was inter-individual (the degree of variation between individuals) and intra-individual (the degree of variation within an individual) variability between repeated measures of flavonoid intake, and intake of flavonoid subclasses was determined in older adults $(n=79)$. Flavonoids and subclasses were assessed using three 4-day weighed food records (WFR) collected approximately 4 months apart. Intra and inter-individual variability was assessed using coefficient of variation (CV) (a standardised measure of dispersion indicating the validity and repeatability of a measure, expressed as a \%). Inter-individual variability was similarly high for each 4-day WFR for total flavonoid intake (mean $\mathrm{CV}=55 \%$ ). The inter-individual variation was similar for flavonoid subclasses of flavonols (mean CV $=45 \%$ ) and flavon-3ols (mean CV $=58 \%$ ), but showed a wider range for flavanones (mean $\mathrm{CV}=102 \%$ ), flavones (mean $\mathrm{CV}=140 \%$ ), and anthocyanins (mean CV $=115 \%$ ). Intra-individual variability between the 3 WFRs was substantial for total flavonoid intake (mean CV $=29 \%$ ), flavan-3ols (mean CV $=34 \%$ ), flavonols (mean CV $=26 \%$ ), flavones (mean $\mathrm{CV}=76 \%$ ), flavanone (mean CV = $77.7 \%$ ), and anthocyanins (mean $\mathrm{CV}=80 \%$ ). These results indicate excessive intra-individual variation associated with estimating flavonoid intake, which has implications for the interpretation study findings that employ a single measurement of intake. It is recommended that future research 
evaluates the number of days of recorded intake required to obtain an accurate estimate of intake of flavonoids and flavonoid subclasses.

\subsection{Fruit and Vegetable Intake and Successful Ageing across Six Countries}

\section{M. Milte, K. E. Lamb and S. A. McNaughton}

Institute for Physical Activity and Nutrition, School of Exercise and Nutrition Sciences, Deakin University, Geelong, Victoria, Australia

Poor dietary behaviours are associated with chronic disease; however, their importance to successful ageing is not well established. This study examines associations between fruit and vegetable intake and successful ageing across six low and middle-income countries (China, Ghana, India, Mexico, Russia, and South Africa). Data from 28,785 participants aged $\geq 50$ yrs from the WHO Study of Global Ageing were considered. Successful ageing profiles were defined based on the following characteristics: participants without chronic disease, cognitive impairment, and depressive symptoms or disability and with good physical; cardiovascular and respiratory function were considered to have successfully aged. Country-specific associations between fruit and vegetable intake (serves per day) and successful ageing were examined using log-binomial regression. The prevalence of successful ageing ranged from $4 \%$ in Mexico to $15 \%$ in China. Preliminary findings showed a positive association between the number of serves of fruit consumed and successful ageing in China ( $R R=1.04,95 \%$ CI: 1.02-1.06), India $(1.19,1.03-1.38)$, and Russia $(1.14,1.02-1.28)$ but a negative association in Ghana $(0.86,0.76-0.97)$. There was no association with fruit in Mexico or South Africa. A positive association was observed between vegetable intake and successful ageing in India $(\mathrm{RR}=1.22,95 \%$ CI: 1.06, 1.40), Mexico (1.40, 1.16-1.70), and Russia (1.18, 1.05-1.33), but a negative association in China $(0.97,0.95-0.99)$, and no associations in Ghana and South Africa. Associations between fruit and vegetable intake and successful ageing are inconsistent, and the influences on successful ageing may vary by region. Studies on understudied populations are needed to meet the challenges of the ageing population.

2.101. A HighWhey Protein, Vitamin D and E Supplement Preserves Muscle Mass, Strength, and Quality of Life in Sarcopenic Elderly

Yacong Bo 1,t, Changfeng Liu 2,t, Xueyuan Zhang 1, Jie You 1, Dandan Duan 1, Yafei Sun 1, Yiwei Zhu ${ }^{1}$, Han Cui ${ }^{1}$ and Quanjun $\mathrm{Lu}{ }^{1, *}$

1 Department of Nutrition and Food Hygiene, College of Public Health, Zhengzhou University, Zhengzhou 450001 China

2 The First Affiliated Hospital of Zhengzhou University, Zhengzhou 450052, China

* Correspondence: lqjnutr@zzu.edu.cn

+ These authors contributed equally to this work.

Objective: Sarcopenia, an age-related loss of muscle mass, strength, and function, poses significant threats to physical performance, independence, and quality of life. The aim of the current study is to test the hypothesis that a targeted nutritional supplement containing whey protein, vitamin $\mathrm{D}$, and vitamin $\mathrm{E}$ can improve measures of sarcopenia.

Methods: A total of 60 sarcopenic elderly people participated in a randomized, double-blind, placebo-controlled supplementation trial for 6 months. Muscle mass (relative skeletal mass index, RSMI), muscle strength (handgrip strength), physical function (6-m gait speed, chair stand test, and timed-up-and-go test, TUG), quality of life, and blood biochemical indexes were evaluated before and after the 6-month intervention.

Results: Compared to placebo group, nutritional supplementation could increase RSMI (mean difference: $0.18 \mathrm{~kg} / \mathrm{m}^{2}, 95 \%$ CI: $0.01-0.35, p=0.040$ ), handgrip strength (mean difference: $2.68 \mathrm{~kg}, 95 \%$ CI: $0.71 \sim 4.65, p=0.009$ ), SF-36 MCS (mean difference: 11.26, 95\% CI:3.86 18.65, $p=0.004$ ), SF-36 PCS (mean difference: 20.21, 95\% CI: 11.30 29.12, $p<0.001$ ), serum IGF-1 (mean difference: $14.34 \mathrm{ng} / \mathrm{mL}$, 95\% CI: 2.06 26.73), and IL-2(mean difference: -575.32 pg/mL, 95\% CI: $-1116.94 \sim-33.70, p=0.038$ ) in the intervention group. 
Conclusion: The current study demonstrated that the combination of whey protein, vitamin $\mathrm{D}$, and vitamin E supplementation can significantly improve muscle mass and strength, quality of life, and anabolic markers such as IGF-I and IL-2 in elderly sarcopenia. Key words: elderly; sarcopenia; whey protein; vitamin D; vitamin $\mathrm{E}$

\subsection{Anti-Inflammatory Effects of Sulforaphane on Primary Monocytes Isolated from Obese Patients}

\section{E. J. Williams, L. Guilleminault, B. S. Berthon, K. J. Baines, T. Wright, C. Karihaloo and L. G. Wood}

Priority Research Centre for Healthy Lungs, Hunter Medical Research Institute, University of Newcastle, NSW, Australia

In obese patients, adipose tissue macrophages (ATMs) release pro-inflammatory cytokines (tumor necrosis factor (TNF)- $\Phi \# 177$; interleukin (IL)-1 $\beta$ and IL-6) resulting in systemic inflammation. Systemic inflammation is a key feature of obesity and contributes to the development of comorbidities including cardiovascular disease, type 2 diabetes, and cancer. Sulforaphane is a dietary antioxidant with anti-inflammatory properties and is naturally found in cruciferous vegetables such as broccoli, brussel sprouts, cabbage, and cauliflower. This study examined the effects of sulforaphane on peripheral blood monocytes, the progenitor cells of ATMs. Primary monocytes isolated from peripheral blood of obese patients $\left(B M I \geq 30 \mathrm{~kg} / \mathrm{m}^{2}\right)$ undergoing sleeve gastrectomy (n $=10$ ) were pre-treated with control (DMSO) or sulforaphane $(40 \mathrm{M})$ for $3 \mathrm{~h}$ before being stimulated with lipopolysaccharide (LPS; $1 \mathrm{ng} / \mathrm{mL}$ ) for $15 \mathrm{~h}$. Cell culture supernatants were then collected and assayed for TNF- $\$ 177$ and IL-1 $\beta$ using enzyme-linked immunosorbent assay (ELISA). Pre-treatment with sulforaphane significantly reduced monocyte production of TNF- $\Phi \# 177,(1162(932,1342)$ $\mathrm{pg} / \mathrm{mL}$ (control) versus $110(51,262)) \mathrm{pg} / \mathrm{mL}$ (sulforaphane); $p=0.002)$ and IL-1 $\beta(114(88,283) \mathrm{pg} / \mathrm{mL}$ versus $18(5,38) \mathrm{pg} / \mathrm{mL} ; p=0.008)$. In conclusion, sulforaphane pre-treatment reduced LPS-induced inflammatory cytokine production in monocytes from obese patients. Therefore, increasing the consumption of cruciferous vegetables that contain sulforaphane, or supplementing sulforaphane, may help to reduce the risk of obesity-induced chronic disease.

\subsection{Effects of Intragastric Tryptophan on the Blood Glucose Response to a Nutrient Drink, and Acute Energy Intake, in Healthy Men}

\section{S. S. Ullrich, P. C. E. Fitzgerald, P. Giesbertz, R. E. Steinert, M. Horowitz and C. Feinle-Bisset}

Adelaide Medical School, University of Adelaide, Adelaide, Australia; '2Department of Nutritional Physiology, Technical University of Munich, Freising, Germany

In healthy humans, intraduodenal infusion of L-tryptophan stimulates plasma cholecystokinin and pyloric pressures, and decreases energy intake (EI), and intragastric administration slows gastric emptying (GE). Whether intragastric L-tryptophan (i) lowers the blood glucose (BG) response to a mixed-nutrient drink, given it slows GE, and/or (ii) maintains its EI-suppressant effect, and (iii) whether effects of L-tryptophan are mediated primarily peripherally or centrally, is unknown. 16 healthy participants received, on three occasions, intragastric infusions of $1.5 \mathrm{~g}$ ("tryptophan-1.5 g") or $3 \mathrm{~g}$ ("tryptophan-3 g") L-tryptophan, or control, and 15 min later consumed a mixed-nutrient drink (400 kcal, 56 g carbohydrates). GE ( ${ }^{13} \mathrm{C}$-acetate breath-test), BG, plasma C-peptide (reflecting insulin release), glucagon, cholecystokinin, and tryptophan concentrations were measured for $60 \mathrm{~min}$. EI was assessed immediately afterwards. L-tryptophan dose-dependently increased plasma tryptophan $(\mathrm{r}=$ $0.943, p<0.001)$. Tryptophan-3g, but not tryptophan- $1.5 \mathrm{~g}$, slowed GE $(p<0.05)$, reduced C-peptideauc $(p=0.05)$, and increased glucagonauC $(p<0.001)$, compared with control. L-tryptophan did not reduce $\mathrm{BG}$ in response to the drink. BGiAuC correlated weakly with GEAUC $(\mathrm{r}=0.322, p=0.072)$. L-tryptophan did not stimulate cholecystokinin. While there was no significant overall effect on EI, tryptophan-3g markedly reduced EI in 8/16 participants (vs. control: $-311 \pm 29 \mathrm{kcal},-35 \pm 8 \%, p<0.01$ ), which correlated inversely with plasma tryptophanAUC (all: $\mathrm{r}=-0.466, p<0.01$; reduced EI: $\mathrm{r}=-0.751, p<$ 0.001 ), and directly with GE (all: $r=0.348, p<0.05$; reduced EI: $r=0.536, p<0.05$ ). In conclusion, Ltryptophan's lack of effect on BG may be because effects of glucagon on glucose outweighed those 
resulting from slowed GE. Gastrointestinal mechanisms (GE, cholecystokinin) appear to play a minor role in EI regulation by L-tryptophan, suggesting L-tryptophan acts centrally (via increased plasma levels).

\subsection{Taste Sensitivity to Glucose is Linked to Body Fat Percentage and Body Mass Index in New Zealand European and Pacific Women}

\section{S. Kindleysides, R. Kruger and B. H. Breier}

School of Food and Nutrition, College of Health, Massey University, Auckland, New Zealand

Taste is a strong driver of food selection, and the physiological impairment of sweet taste perception may impact long-term dietary intake. The aim of this study was to determine if taste sensitivity was related to body fat or body composition in women. Healthy, premenopausal, postmenarche New Zealand European and Pacific women (age 18-45 years) were recruited as part of the larger PROMISE (PRedictors linking Obesity and gut MIcrobiomE) study. A total of 257 women, median age 26 years (range 22, 35), underwent a sensory evaluation session in a fasted state. Participant's weight, height, body fat percentage, and waist and hip circumference were measured. Participants completed a non-subjective sweet taste ranking task of four distinct concentrations of glucose dissolved in distilled water, which required ordering solutions from the lowest to the highest concentration by taste alone. Preliminary findings from the study show that approximately $20 \%$ ( $n=$ 51) of the participants were unable to correctly rank the taste solutions. The participants who successfully completed the ranking task had a significantly lower body mass index (BMI) in comparison to participants who were incorrect (median $27.2[22.7,34.2]$ vs. $32.4[24.8,36.8]$, respectively; $p<0.01)$, body fat percentage $(34.7[26.6,43.4]$ vs. $40.1[33.3,44.7], p<0.05)$, waist circumference $(81.3[73,96.5]$ vs. $94.3[78.3,105.5], p<0.01)$, and hip circumference $(107[99.3,120]$ vs. $117[101.2,124.6], p<0.05)$. These findings suggest a link between body composition and impaired taste perception. The study highlights the importance of the relationships between the gustatory system, body weight, and adiposity.

\subsection{Effectiveness of Lifestyle Interventions for Preventing Weight Gain among at-Risk Young Adults: A Systematic Review}

\section{N. Hayba, S. R. Partridge, M. M. Nour, A. Grech and M. Allman Farinelli}

The University of Sydney, Nutrition and Dietetics Group, School of Life and Environmental Science, Charles Perkins Centre, Sydney, NSW 2006, Australia

The incidence of overweight and obesity are increasing with each successive generation of young adults. Associated co-morbidities will emerge at an earlier age unless weight gain is prevented. Evidence has demonstrated young adults (aged 18-35 years) from low socioeconomic and ethnically diverse backgrounds are at greater risk of overweight or obesity, yet it is unclear how to effectively intervene in this population. This systematic review aimed to assess the effectiveness of lifestyle interventions conducted in this population. Nine electronic databases were searched from January 1980 until May 2017 for studies reporting on lifestyle interventions for prevention of weight gain and obesity. The main outcome was weight change and secondary outcomes were diet and physical activity. Thirty studies met the inclusion criteria. Six interventions included subgroup analyses to determine if ethnicity moderated weight change and/or secondary outcomes, and two included subgroup analyses to determine if socioeconomic status had an effect on change in weight. Five of these six studies were effective in preventing weight gain, and subgroup analyses showed no differences in effect by ethnicity. Of these five studies, two included a subgroup analysis that showed socioeconomic status to have no effect on weight outcome. Of the six studies, two included a subgroup analysis that showed ethnicity had no effect on improved diet and physical activity outcomes. Despite the promising results from these five lifestyle interventions utilizing online and mobile components to effectively reach and prevent weight gain in this priority population, the evidence base of high quality trials is limited. 


\title{
2.106. Evaluating a Flexible Meal Replacement Program for Weight Loss, Retention, and Health Outcomes
}

J. Bowen, E. Brindal, G. James-Martin and M. Noakes

CSIRO Health \& Biosecurity, Adelaide, Australia

Meal replacements are an effective strategy for weight loss; however, success can be limited by retention. Small studies have shown that alternate day fasting (ADF) can achieve similar weight loss and metabolic improvements to continuous energy restriction (CER). This study aimed to explore whether combining ADF with the use of meal replacements improves retention, weight loss, and markers of metabolic health, compared to a CER meal replacement weight loss program. 164 healthy adults (25-60 years) with a BMI $>27 \mathrm{~kg} / \mathrm{m}^{2}$, were randomised to the standard meal replacement program (S-MRP) using CER or a modified meal replacement program (M-MRP) incorporating 3 days of 'modified fasting' and one day of 'free eating' per week. Participants followed the diets for 16 weeks with fortnightly support from a dietitian, followed by 8 weeks of weight maintenance (healthy eating plan, no support). Retention was high in both S-MRP and M-MRP groups (83 and $82 \%$ respectively, NS between groups). There was no significant difference in weight loss between groups at week $16(-10.7 \pm 0.5 \mathrm{~kg}$ S-MRP; $-11.2 \pm 0.6 \mathrm{~kg}$ M-MRP time effect $p<0.01)$ or at week 24 $(-10.8 \pm 0.7 \mathrm{~kg}$ S-MRP; $-9.7 \pm 0.6 \mathrm{~kg}$ M-MRP time effect $p<0.01)$. Both groups had significant improvements in blood lipids (cholesterol, LDL, triglycerides), blood pressure, fasting insulin and glucose, folate, vitamin B12, and ferritin status; and ratings of mood, food cravings, and eating restraint at week $16(p<0.05)$. CER and ADF approaches using meal replacements are equally effective strategies for weight loss and improved markers of metabolic health.

\subsection{Effect of an Interdisciplinary Intervention with Individualized Dietary Advice: Weight Change in the HealthTrack Study}

\author{
L. C. Tapsell 1,2, E. P. Neale ${ }^{1,2}$, A. Martin 1,2, B. Thorne ${ }^{1,2}$ and M. J. Batterham 4 \\ 1 Faculty of Science, Medicine and Health, University of Wollongong, NSW, Australia \\ 2 Illawarra Health and Medical Research Institute, University of Wollongong, NSW, Australia \\ 3 Faculty of Engineering and Information Sciences, University of Wollongong, NSW, Australia
}

Evidence supports interdisciplinary efforts in diet, exercise, and behavior to achieve weight loss in clinical care. For the dietary component, research indicates that negotiations on the type of foods consumed may be as important as the amounts. The aim of this analysis was to report on differences in weight change over 12 months in the HealthTrack trial that tested the effects of an interdisciplinary approach (including individualised dietary advice) versus usual care (general advice). Participants $(\mathrm{N}=377)$ were randomized to control $(C)$, intervention $(I)$, or intervention + a healthy food supplement, $30 \mathrm{~g}$ walnuts/day $(I W)$ groups. A mixed model approach found significant differences in weight loss $(p<0.001)$. After the 3 month intensive phase, the \% weight change in the intervention groups was greater than the controls $(1.4+2.7$ vs. $2.6+3.2 \mathrm{I}$ and $2.7+3.8 \mathrm{IW})$ and remained so at 12 months $(1.9+3.8$ vs. $3.8+5.7 \mathrm{I}$ and $3.8+6.6 \mathrm{IW})$. As greater within group variation occurred at 12 months, the weight loss effects were only statistically significant at 3 months, and at 6 months between $C$ and IW. Focusing on individual foods within a weight loss intervention accommodates knowledge of food choices that enable an energy deficit, but more intensive behavioral support appears required to sustain the effort.

\subsection{Weighing Attitudes and Behaviour among Pregnant Women}

\section{J. C. Willcox ${ }^{1}$, K. J. Campbell ${ }^{2}$, K. Ball ${ }^{2}$ and P. van der Pligt ${ }^{2}$}

1 School of Exercise and Nutrition Sciences, Deakin University, Melbourne, Australia

2 Institute for Physical Activity and Nutrition Research, Deakin University, Melbourne, Australia

Promoting healthy gestational weight gain has become a key component of antenatal clinical guidelines. This study aimed to explore pregnant women's self-weighing practices and views on 
health professional $(\mathrm{HP})$ weighing. Consecutive attendees $(n=1046)$ received a questionnaire after their first antenatal visit to a tertiary Melbourne maternity hospital. Demographics, frequency of selfweighing, and agreement on a five point Likert scale for " $\mathrm{I}$ ' $\mathrm{m}$ embarrassed when a doctor or midwife weighs me" were assessed. Participants $(n=368 ; 35 \%$ response) averaged 32.5 years, 20 weeks gestation, and $35 \%$ speaking a LOTE. Thirty-six percent had a pre-pregnancy BMI (ppBMI) in the overweight/obese categories, with $59 \%$ in the healthy range. $40 \%$ of women self-weighed monthly, $16 \%$ fortnightly, $26 \%$ weekly or more frequently, and $18 \%$ never. Women with a tertiary education were nearly three times more likely to self-weigh (OR 2.8; 95\% CI 1.26-6.31) than those without. 63\% of women "disagreed" or "strongly disagreed" that they were embarrassed by HP weighing, while $20 \%$ neither "agreed nor disagreed" and 17\% "agreed or strongly agreed". Women with overweight and obese ppBMIs were more than three (OR 3.49; 95\% CI1.66-7.36) and thirteen times (OR 13.74; 95\% CI 6.41-29.44) more likely to agree to embarrassment than those with a healthy weight. In conclusion, the majority of pregnant women reported self-weighing at least once per month, with tertiary educated women three times more likely to self-weigh. While the majority of women (83\%) did not report embarrassment with HP weighing, those who were overweight or obese prior to pregnancy were significantly more likely to report discomfiture.

\subsection{Dose-Dependent Effects of LCn3PUFA on Fat Oxidation and Resting Energy Expenditure in Overweight Females: A Pilot Study}

\section{A. M. Hill ${ }^{1}$, L. C. Shore ${ }^{2}$, S.-Y. Tan ${ }^{1,3}$ and A. M. Coates ${ }^{2}$}

1 School of Pharmacy and Medical Sciences, University of South Australia, Adelaide, Australia

2 School of Health Sciences, University of South Australia, Adelaide, Australia

3 Clinical Nutrition Research Centre, Singapore Institute for Clinical Sciences (SICS), Agency for Science, Technology and Research (A*STAR), Singapore

Several animal and human studies have investigated whether long chain omega 3 polyunsaturated fatty acids (LCn3PUFA) can reduce adiposity, with inconsistent results. Doseresponse studies suggest that body weight, age, and gender may mediate this relationship. It therefore remains unclear whether a single-dose or "tailored" doses would be more efficacious. This study aimed to evaluate the effects of LCn3PUFA, administered in a dose-dependent manner based on body mass index (BMI), on resting energy expenditure (REE), fat oxidation, and body fat in overweight females. We conducted a double-blind, randomised, placebo-controlled, parallel study. Twenty-three overweight/obese females consumed fish oil (FO; $n=12)$ or placebo corn oil $(\mathrm{CO} ; \mathrm{n}=$ 11) for 6 weeks. FO dose was based on BMI, with participants consuming LCn-3PUFA equivalent to $1200 \mathrm{mg} / \mathrm{d}$ (BMI $25.0-27.4 \mathrm{~kg} / \mathrm{m}^{2}$ ), $1800 \mathrm{mg} / \mathrm{d}$ (BMI $27.5-29.9 \mathrm{~kg} / \mathrm{m}^{2}$ ), $2400 \mathrm{mg} / \mathrm{d}$ (BMI 30.0-32.4 kg/m²), or $3000 \mathrm{mg} / \mathrm{d}\left(\mathrm{BMI} \geq 32.5 \mathrm{~kg} / \mathrm{m}^{2}\right.$ ). REE, fat oxidation, weight, and body composition were measured at baseline and 6 weeks. No significant treatment $x$ time interactions were observed for all variables. There was a significant inverse relationship between dose of LCn3PUFA per kilogram body mass and changes in REE $(r=-0.661, p=0.038$, ) and fat oxidation $(r=-0.636, p=0.048)$. Contrary to our hypothesis, in this small sample, the FO supplemented group did not experience an increase in REE and fat oxidation or reduction in body fat compared with the placebo group over 6 weeks.

\subsection{Differences of Adiponectin Expression in High-Fat Dietary Induced Obesity and Obesity-Resistant} Rats

L. Mao ${ }^{1}, \mathrm{H}$. Zhang ${ }^{2}$, M. $\mathrm{Hu}^{1}$ and $\mathrm{Y} . \mathrm{Li}^{2}$

1 Department of Nutrition and Food Hygiene, School of Public Health, Southern Medical University, China

2 Tongji Medical College of HUST, China

Hypoadiponectinemia is associated with obesity, diabetes, metabolic syndrome, and many other diseases. This study aims to investigate the effects of high-fat diet (HFD) on adiponectin in rats with different susceptibility to obesity. A total of 80 Male Sprague-dawley (SD) rats were divided into control (10) and HFD (70) group, respectively, fed with basic diet for 20 weeks and HFD for 12 weeks. 
HFD induced obesity (DIO) and obesity-resistant (DIO-R) rats were selected randomly by body weight (BW) and were continuously fed with HFD for another 8 weeks. Food ingestion, BW and fat mass were recorded. Serum adiponectin, lipid profiles, fasting glucose (FG), insulin, and adiponectin mRNA in visceral adipose tissues were measured. BW, visceral adipose weight, fat percentage, and energetic efficiency in DIO rats were higher than those in DIO-R and control rats $(p<0.05)$. Although serum HDL-C levels in DIO and DIO-R rats were lower than the control $(p<0.05)$, serum TC, TG, FG, and LDL and insulin levels in DIO rats were higher than DIO-R rats $(p<0.05)$. DIO rats rather than DIO-R rats showed a lower adiponectin mRNA expression than that in the control $(p<0.05)$. DIO rats have a lower serum adiponectin level than DIO-R rats $(p<0.05)$. Furthermore, results of correlation analysis demonstrated that serum adiponectin was negatively correlated to BW and insulin $(p<0.05)$ but positively correlated to FG $(p<0.05)$. In conclusion, adiponectin levels were different between DIO and DIO-R rats, and the level of adiponectin may play a critical role in the development of different susceptibility to obesity.

\title{
2.111. Dietary Supplement Use during Preconception and Pregnancy: The Australian Longitudinal Study on
} Women's Health

\section{E. McKenna ${ }^{1}$, A. J. Hure ${ }^{2}$, A. Perkins ${ }^{3}$ and E. Gresham ${ }^{1}$}

1 School of Allied Health Science, Griffith University, QLD, Australia

2 School of Medicine and Public Health, University of Newcastle, and Hunter Medical Research Institute, NSW, Australia

3 School of Medical Science, Griffith University, and Menzies Health Institute, QLD, Australia

Worldwide, dietary supplement use among reproductive aged women is becoming increasingly common. The aim of this study was to investigate and report on dietary supplement use in Australia during preconception and pregnancy. Self-reported data were collected prospectively for the Australian Longitudinal Study on Women's Health (ALSWH). The sample included 1323 women aged 31-36 years, classified as preconception $(n=485)$ or pregnant $(n=838)$ when completing Survey 5 of the ALSWH in 2009, with supplementation data. Frequency and contingency tables were calculated and Pearsons chi square test for associations between demographic variables and supplementation status was performed. Supplement use was higher during pregnancy $(81 \%)$ compared to women who were preconception (63\%). Multiple-micronutrient supplements were the most commonly reported supplements (44\% preconception vs. $67 \%$ pregnant women). Supplements containing folic acid ( $51 \%$ vs. $74 \%$ ) and iodine (37\% vs. $60 \%$ ) were also common during preconception and pregnancy, respectively. Folic acid, omega-3 fatty acids, vitamin C, B vitamins, iron, and calcium were the most common single nutrients supplemented. Women trying to fall pregnant, with no previous children, born outside of Australia, living in urban areas, with a higher household income $(>\$ 130,000 \mathrm{pa})$, who were non-smokers with a healthy Body Mass Index were more likely to take dietary supplements. Dietary supplement use before and during pregnancy in Australia is relatively high; however, clear disparities between nutrients supplemented and nutrients recommended for supplementation exist. Further research is required to determine the role of dietary supplementation on pregnancy and birth outcomes.

\subsection{Postprandial Lipidomic Profiles after Krill Oil Supplementation Compared with Fish Oil in Healthy} Women

\author{
H. H. Sung ${ }^{1}$, A. J. Sinclair ${ }^{2,3}$, N. Mellett ${ }^{4}$, P. J. Meikle ${ }^{4}$ and X. Q. Su ${ }^{1}$ \\ College of Health and Biomedicine, Victoria University, Melbourne, Australia \\ 2 School of Medicine, Deakin University, Geelong, Australia \\ 3 Department of Nutrition, Dietetics and Food, Monash University, Nottinghill, Australia \\ 4 Baker Heart and Diabetes Institute, Melbourne, Australia
}

There is continuing controversy as to whether krill oil (KO) is more bioavailable compared with fish oil (FO). This study aimed to compare the postprandial plasma lipidomic profiles after consumption of $\mathrm{KO}$ and $\mathrm{FO}$. In a randomised cross-over study, $5 \mathrm{~g}$ of each of $\mathrm{KO}$, FO, or olive oil 
(OO), mixed in a test meal of fresh mashed potato containing $15 \mathrm{~g}$ of $\mathrm{OO}$, was randomly consumed by 10 healthy women (18-45 years) on each study day with seven days wash-out period between. Plasma lipidomic profiles $(\mathrm{pmol} / \mathrm{mL})$, conducted using electrospray-ionisation tandem mass spectrometry, were analysed at baseline, 3 , and 5 using repeated-measure ANOVA. The significant differences $(p<0.05)$ at $5 \mathrm{~h}$ between EPA-containing molecular species resulting from KO and FO consumption were analysed using paired t-test. There was a significant increase in EPA molecular species following $\mathrm{KO}$ and FO consumption over the 5-h postprandial period. Eight of 14 TAG-EPA were significantly increased from the baseline to $5 \mathrm{~h}$ by 2-5x, and six of seven DAG-EPA by 2-7x for KO, compared with 13/14 TAG-EPA (3-154x) and 7/7 DAG-EPA (4-61x) for FO. 7/14 TAG-EPA and 6/7 DAG-EPA for FO were significantly higher than $\mathrm{KO}$ at $5 \mathrm{~h}$, whereas for 20 phospholipid-EPA species detected, 12/20 for KO were significantly higher than FO at $5 \mathrm{~h}$. The trends of lipidomic changes in TAG, DAG, and PL species were significantly different between $\mathrm{KO}$ and FO consumption, thus indicating that in the postprandial phase EPA from $\mathrm{KO}$ and FO are differentially incorporated into EPA-molecular species from these lipid classes.

\subsection{Adherence to Omega-3 Fatty Acid Supplementation in a Randomized Trial in Lung Transplant} Recipients

K. Miura ${ }^{1}$, D. C. Chambers ${ }^{2,3}$, P. Hopkins ${ }^{4}$, A. Vail 5, L. E. Rhodes ${ }^{6}$, H. P. Soyer ${ }^{7,8}$ and A. C. Green ${ }^{1,9}$

1 Cancer and Population Studies Group, QIMR Berghofer Medical Research Institute, 300 Herston Road, Herston, QLD 4006, Australia

2 Queensland Lung Transplant Service, The Prince Charles Hospital, Brisbane, Australia

3 School of Medicine, The University of Queensland, Brisbane, Australia

${ }_{4}$ Queensland Lung Transplant Service, TPCH campus and School of Medicine, University of Queensland, Brisbane, Australia

5 Centre for Biostatistics, Institute of Population Health, University of Manchester, Manchester Academic Health Science Centre, Salford Royal NHS Foundation, Trust, Manchester, UK

6 Dermatology Research Centre, School of Biological Sciences, Faculty of Biology Medicine and Health, University of Manchester, Manchester Academic Health Science Centre, Salford Royal NHS Foundation Trust, Manchester, UK

7 Dermatology Research Centre, The University of Queensland; The University of Queensland Diamantina Institute, Translational Research Institute, Brisbane, Australia

8 Department of Dermatology, Princess Alexandra Hospital, Brisbane, Australia

9 CRUK Manchester Institute, University of Manchester, Wilmslow Road, Manchester M13 9PL, UK

The risk of skin cancer is greatly increased among lung transplant recipients. Omega-3 fatty acids, especially eicosapentaenoic acid (EPA) and docosahexaenoic acid (DHA) found in oily fish, may reduce this risk. In a randomized double-blind pilot trial, we assessed adherence to omega-3 fatty acid or placebo supplements for skin cancer prevention in lung transplant recipients, the first trial of its kind. In 2014-2015, eligible lung transplant recipients ( $\geq$ one year post-transplant with stable graft) were recruited from The Prince Charles Hospital, the thoracic transplant center for Queensland. Patients were randomly allocated to take four capsules daily containing $3.6 \mathrm{~g}$ total omega-3 (46\% EPA, 38\% DHA) or $4 \mathrm{~g}$ olive oil (placebo) and were followed for 12 months. Adherence was assessed by comparing the median plasma EPA at end of intervention with baseline levels. Of the 49 participants who consented (median age 55 years, $67 \%$ males), one died, six withdrew, and eight did not provide final blood samples, resulting in 34 (18 active, 16 placebo) bloods for analysis. After 12 months, plasma EPA increased substantially from $123.5 \mathrm{~mol} / \mathrm{L}$ to $340.0 \mathrm{~mol} / \mathrm{L}$ in the intervention group (median change +163 , range -392.0 , 406.0) compared with the placebo group (median change $+18 \mathrm{~mol} / \mathrm{L}$, range $-94.0,102.0)(p=0.004)$. Eight patients (three active, five placebo) did not take capsules ("too many"). Overall, however, the majority of lung transplant recipients adhered to the intervention, with large increases in blood omega-3 fatty acids in the active treatment group, suggesting reasonable adherence can be expected in the definitive trial. 


\title{
2.114. Does Omega-3 Rich Fish Oil Supplementation during Fetal Brain Growth Improve Child Brain
} Development?

\author{
J. Gould ${ }^{1}$, K. Treyvaud ${ }^{2}$, P. Anderson ${ }^{3}$ and M. Makrides ${ }^{1}$ \\ 1 South Australian Health and Medical Research Institute, Australia \\ 2 La Trobe University, Australia \\ 3 Monash University, Australia
}

The purpose of this study was to determine whether fish oil supplementation during the key period of brain growth improves child brain development. Design: Women were enrolled in a randomised controlled trial of omega-3-rich fish oil supplementation in pregnancy. Child neurobehavioural development was measured in 726 children up to seven years of age, with cognitive development being the primary outcome. Women were either given omega-3-rich fish oil or a placebo during the last half of their singleton pregnancy. Multiple domains of child development were assessed. At 18 months cognition, motor and language were assessed using the Bayleys Scales of Infant and Toddler Development-3rd edition; at four years cognition was assessed using the Differential Ability Scale-2nd edition, along with the assessment of executive functioning (EF), behaviour and language abilities; and at seven years cognition was assessed using the Wechsler Abbreviated Scale of Intelligence-2nd edition, along with the assessement of language, attention, academic abilities, EF, behaviour, ADHD symptoms, verbal learning memory, spatial organisation, and inhibition. There was no effect of fish oil supplementation on the primary outcome at 18 months (adjusted mean difference, $0.01 ; 95 \% \mathrm{CI},-1.36$ to 1.37; $p=0.99$ ), four years (adjusted mean difference $0.29,95 \%$ confidence interval -1.35 to $1.93, p=0.73$ ), or seven years (adjusted mean difference 1.11, $95 \%$ confidence interval -0.67 to $2.90, p=0.22$, preliminary data). Nor were there differences between the executive functioning or language skills, however at four and seven years there were some differences in parent-rated child behaviour. Omega-3-rich fish oil supplements during fetal brain development are unlikely to provide the benefits marketed by supplement manufacturers.

\subsection{Nitrate-Rich Vegetables Do Not Lower Blood Pressure in Individuals with Mildly Elevated Blood Pressure}

L. C. Blekkenhorst ${ }^{1}$, J. R. Lewis ${ }^{1}$, R. L. Prince ${ }^{1}$, A. Devine ${ }^{2}$, N. P. Bondonno ${ }^{1}$, C. P. Bondonno ${ }^{1,2}$, L. G. Wood ${ }^{3}$, I. B. Puddey ${ }^{1}$, N. C. Ward ${ }^{1}$, K. D. Croft ${ }^{1}$, R. J. Woodman ${ }^{4}$, J. O. Lundberg ${ }^{5}$, L. J. Beilin ${ }^{1}$ and J. M. Hodgson ${ }^{1,2}$

1 School of Medicine and Pharmacology, University of Western Australia, Perth 6000, Australia

2 School of Medical and Health Sciences, Edith Cowan University, Joondalup WA 6027, Australia

3 School of Biomedical Science and Pharmacy, University of Newcastle, Callaghan NSW 2308, Australia

4 Flinders Centre for Epidemiology and Biostatistics, Flinders University, Adelaide 5000, Australia

5 Department of Physiology and Pharmacology, Karolinska Institutet, Solnavägen 1, 17177 Solna, Sweden

Evidence suggests that the consumption of nitrate-rich vegetables may be effective in reducing blood pressure. Our primary aim was to determine whether increased consumption of nitrate-rich vegetables would result in lower blood pressure. Thirty participants with pre-hypertension or untreated grade 1 hypertension were recruited to a randomised controlled crossover trial with fourweek treatment periods. Participants completed three treatments in random order: (1) increased intake of nitrate-rich vegetables (high nitrate, $\mathrm{HN}$ ); (2) increased intake of nitrate-poor vegetables (low nitrate, LN); and (3) no increase in vegetables (control, C). The primary outcome was systolic blood pressure (SBP) assessed using 24-h ambulatory, home and clinic measurements. Plasma nitrate and nitrite concentrations were increased with the $\mathrm{HN}$ treatment compared to the $\mathrm{LN}$ and $\mathrm{C}$ treatments $(p<0.001)$. Plasma total carotenoids, lutein and beta-carotene were increased with the HN and LN treatments compared to the $\mathrm{C}$ treatment $(p<0.01)$. HN and LN treatments did not reduce SBP (24-h ambulatory $-\mathrm{HN}: 127.4 \pm 1.1 \mathrm{mmHg}, \mathrm{LN}: 128.6 \pm 1.1 \mathrm{mmHg}, \mathrm{C}: 126.2 \pm 1.1 \mathrm{mmHg}, p=0.200$; home $-\mathrm{HN}$ : $127.4 \pm 0.7 \mathrm{mmHg}$, LN: $128.7 \pm 0.7 \mathrm{mmHg}, \mathrm{C}: 128.3 \pm 0.7 \mathrm{mmHg}, p=0.360$; clinic $-\mathrm{HN}$ : $128.4 \pm 1.3 \mathrm{mmHg}, \mathrm{LN}: 130.3 \pm 1.3 \mathrm{mmHg}, \mathrm{C}: 129.8 \pm 1.3 \mathrm{mmHg}, p=0.492$ ) or diastolic blood pressure. No differences were observed between treatments for central systolic and diastolic pressure, central 
augmentation index and pulse wave velocity. Increased intake of nitrate-rich and nitrate-poor vegetables did not decrease blood pressure in individuals with mildly elevated blood pressure.

\subsection{Impact of Nutrition Education on Iron Nutritional Status of School-Going Adolescent Girls}

Das, Pranati, Khatoniar, Sushmita and Bora, Papori

Department of Food Science and Nutrition, Assam Agricultural University, India

Iron deficiency anaemia is the most prevalent nutritional problem in developing countries, including India. Thus, the present study was conducted to assess the prevalence of anaemia and the impact of nutrition education on the nutritional and haemoglobin status of adolescent girls. A total of 300 school-going adolescent girls from rural areas of Jorhat district, Assam, India, were screened using the cyanmethemoglobin method and found to have a haemoglobin level below the WHO cutoff of $12 \mathrm{gm} / \mathrm{dL}$. A nutrition education intervention was provided for three months and its impact on the nutrition knowledge score, food-nutrient intake and haemoglobin levels was studied using a standardized schedule and diet survey method. A high prevalence of anaemia of $95.54 \%$ was observed in the population studied, indicating a greater health risk for adolescent girls. It was observed that the nutrition education significantly improved the haemoglobin levels of the experimental group from $10.01 \pm 1.08 \mathrm{~g} / \mathrm{dL}$ to $11.18 \pm 0.77 \mathrm{~g} / \mathrm{dL}$. A positive correlation was observed between the haemoglobin level and the intake of pulses, green leafy vegetables, fruits, and milk and milk products, as well as the intake of protein, iron, vitamin $C$ and folic acid. Further nutrition knowledge in the form of knowledge, attitude and practice scores also showed a positive correlation with haemoglobin levels. However, the enhancement of the intake and knowledge scores did not attain complete adequacy or normalcy levels as compared to recommended levels. It can be concluded that a more intense nutrition education programme may be required through the school curriculum for further improvements in the iron nutritional status of adolescent girls.

\subsection{Prevalence of Vitamin D Deficiency in Chinese Children and Adolescents from 2010-2012}

\section{Y. Hu, M. Li, L. Yang and X. Yang}

Key Laboratory of Trace Element Nutrition of National Health and Family Planning Commission, National Institute for Nutrition and Health, Chinese Center for Disease Control and Prevention, Beijing 100050, China

Objective: The prevalence of vitamin D deficiency was evaluated by analyzing the levels of vitamin D in Chinese children and adolescents aged 6-17 years in 2010-2012.

Methods: All the data in this study were from the China Nutrition and Health Survey 2010-2012. Using multi-stage stratified sampling and the population proportional stratified random sampling method, 14,434 children from 75 cities and 75 rural areas were included in this study. Information on the basic situation of the survey object was collected by questionnaire survey. The vitamin D level was determined by enzyme-linked immunosorbent assay and vitamin D deficiency was judged according to the recommendation standards from the National Institute of Medicine, National Academy of Sciences. The levels of serum 25 hydroxyvitamin D of the different age groups were compared, and the differences in the prevalence of vitamin D deficiency rates (including deficiency and serious deficiency) were also compared.

Results: The vitamin D level of Chinese children and adolescents was $20.48 \pm 8.44 \mathrm{ng} / \mathrm{mL}$ and $20.57 \pm 8.40 \mathrm{ng} / \mathrm{mL}$ for cities and $20.38 \pm 8.49 \mathrm{ng} / \mathrm{mL}$ for rural areas. The prevalence of Vitamin D deficiency in Chinese children and adolescents was up to $53.22 \%$ and $53.78 \%$ for cities and $52.78 \%$ for rural areas.

Conclusion: The prevalence of vitamin D deficiency in Chinese children and adolescents was generally more serious than in adults, more attention and improvement measures should be taken to encourage them participate in more outside activities. 


\subsection{Low Dietary Zinc Intake Is Associated with High Psychological Distress in Japanese Workers}

M. Nakamura, A. Miura, A. Toki, Y. Shibata, E. Okada and T. Ojima

Affiliation not provided.

Mental health disorders are one of the important health concerns for workers. To explore the primary prevention strategy, the association between dietary zinc intake and psychological distress was investigated. A questionnaire survey was conducted at 43 companies in 2013-14. Dietary intake was estimated by a validated food frequency questionnaire for Japanese. The total energy intake was adjusted by residual methods. Psychological distress was assessed using the six-item Kessler Psychological Distress Scale (K6). High psychological distress was determined by a score of 13 and more. Sex and age adjusted means and 95\% confidence intervals (CIs) for zinc intake and psychological distress were obtained by analysis of covariance. A logistic regression analysis was performed to obtain odds ratios (ORs) and 95\% CIs for high psychological distress according to quartile of zinc intake while adjusting for age and sex, with the highest quartile (Q4) as a reference. Valid answers for sex and K6, age 18 to 70, total energy intake within 500-4000 kcal/day, and no history of depression were obtained from 2089 men and women. The proportion of high psychological distress was $8.1 \%$ for men and $4.2 \%$ for women. Mean zinc intakes were $7.50 \mathrm{mg}(95 \%$ CI, 7.34-7.67) for high psychological distress and $7.84 \mathrm{mg}$ (7.80-7.89) for low psychological distress. ORs for high psychological distress were 1.75 (1.07-2.87) for the lowest (Q1) quartile of zinc intake, $1.26(0.75-2.11)$ for Q2 and $0.80(0.46-1.41)$ for Q3. Low dietary zinc intake is associated with high psychological distress. Further research on the association should be encouraged.

\subsection{Multiple Micronutrient Deficiencies Persist Among Young Children in Malaysia}

\section{G. Khor, S. Shyam, S. Misra and M. Chong}

Department of Nutrition and Dietetics, International Medical University, Kuala Lumpur 57000, Malaysia

Malaysia has undergone steady economic growth, industrialization and technological development to become an upper-middle income economy. Occurring in tandem has been an improvement in the nutritional status of young children in terms of anthropometric indicators. Nonetheless, the results on dietary intake and from blood assessments in recent years have revealed that multiple micronutrient deficiencies prevail in young children. A nationally representative study reported a noteworthy prevalence of inadequate intake of calcium, iron, and vitamins A and D among children aged 0.5-6.9 years. Based on two-day weighed food records, the dietary intake of calcium, iodine, zinc, selenium, niacin, folate, and vitamins C, A, D and E were lower than the recommended levels among toddlers aged 15-21 months from urban communities. Using a similar assessment approach for subjects aged 0.5-23.9 months, an inadequate intake of zinc, thiamin, riboflavin, niacin, and vitamins $\mathrm{A}$ and $\mathrm{C}$ was found. Of the few dietary studies that included blood assessments, notably high serum vitamin D insufficiency was recorded in preschool and school-aged children of both sexes, whilst iron deficiency anaemia prevailed in female children from rural areas. These findings occurred in the face of various child health improvement programmes in communities, schools, preschools, day care centres and clinics. The National Plan of Action for the Nutrition of Malaysia (NPANM, 2016-2025) includes several strategies and interventions to address micronutrient deficiencies in children. There is a need for more rigorous monitoring and outcome-based evaluation of public health programmes, and research for a better understanding of the existence of multiple micronutrient deficiencies among young children in an affluent nation.

2.120. Self-Compassion Interventions for Nutrition Habits, Eating Behaviour, Body Weight and Body Image: A Systematic Review

\footnotetext{
H. Rahimi-Ardabili 1, L. R. Vartanian ${ }^{2}$, R. C. Reynolds ${ }^{1}$, L. V. McLeod ${ }^{2}$ and N. Zwar 1,3

1 School of Public Health and Community Medicine, University of New South Wales, NSW 2052, Australia

2 School of Psychology, University of New South Wales, NSW 2052, Australia
} 
3 School of Medicine, University of Wollongong, Wollongong NSW 2522, Australia

Overweight and obesity are universal health challenges, with behavioural weight-management often failing to produce long-term effects. Various psychological factors such as body dissatisfaction and disordered eating have been linked to weight gain over time; however, the majority of weight loss programs have not addressed these factors. There has been a growing interest in the potential benefits of self-compassion as a new approach to promoting both physical and mental health. This systematic review investigates the effect of interventions that aim to increase self-compassion in obesity and weight-related psychological conditions. Four electronic databases were searched, adapting search terms from previous systematic reviews on nutrition and body weight, compassion, eating disorders and body image. This review was conducted using the PRISMA guidelines for systematic reviews. The search identified six studies that met the eligibility criteria for the review. Results show that self-compassion can be beneficial for weight loss, nutrition behaviours, eating behaviour and body image. However, the numbers of studies are limited, and most of the studies have serious limitations. Further research with a robust methodology is needed to determine the efficacy of self-compassion on behaviour that is related to obesity.

\subsection{Relationship between Erythrocyte Membrane Phospholipid Fatty Acids and Obesity in Chinese Children and Adolescents}

\section{J. Tang ${ }^{1}$, Y. K. Yan ${ }^{2}$, J. Li ${ }^{3}$, B. Yang ${ }^{4}$, X. Y. Zhao ${ }^{2}$, Z. H. Li ${ }^{1}$, X. F. Guo ${ }^{1}$, J. S. Zheng ${ }^{1,4,5}$, J. Mi $^{2}$ and D. $\mathrm{Li}^{1,4}$}

1 Department of Food Science and Nutrition, Zhejiang University, Hangzhou, China

2 Department of Epidemiology, Capital Institute of Pediatrics, Beijing, China

3 Section 1, Department of Nutrition, Chinese PLA General Hospital, Beijing, China

4 Institute of Nutrition \& Health, Qingdao University, Qingdao, China

5 Medical Research Council Epidemiology Unit, University of Cambridge, Cambridge, UK

The association between circulating fatty acid (FA) composition and obesity risk remains largely unclear in children and adolescents. We conducted a case-control study of 1442 pairs of obese and normal-weight children and adolescents matched by age and sex. Multivariable-adjusted odds ratios (ORs) and 95\% confidence intervals (CIs) of obesity risk for per one standard deviation (SD) difference for each FA were estimated by fitting conditional logistic regression models. A literaturebased meta-analysis was additionally conducted to compare the FA patterns in different body weight groups from our findings with summary results from existing evidence. In the present study, the cases showed higher proportions of five saturated FAs (SFAs) (14:0, 16:0, 17:0, 18:0, 20:0), but lower proportions of all n-3 polyunsaturated FAs (PUFAs) (18:3n-3, 20:3n-3, 20:5n-3, 22:5n-3, 22:6n-3) than controls, summary mean group differences from the meta-analysis were only consistent in 22:5n-3 and 22:6n-3. In the conditional logistic regression models, five SFAs (14:0, 16:0, 17:0, 18:0, 20:0) were significantly positively associated with obesity risk, with per one SD OR $(95 \% \mathrm{CI})$ as $1.22(1.12,1.32)$, $1.43(1.32,1.57), 1.13(1.07,1.20), 1.08(1.03,1.13)$ and $1.33(1.22,1.44)$, respectively. All n-3 PUFAs were significantly inversely associated with obesity risk, the per one SD OR $(95 \% \mathrm{CI})$ were $0.81(0.71,0.91)$ for 18:3n-3, $0.70(0.61,0.80)$ for 20:3n-3, $0.69(0.60,0.79)$ for 20:5n-3, $0.60(0.52,0.69)$ for $22: 5 n-3$ and $0.57(0.51,0.64)$ for $22: 6 n-3$, respectively. The present study found that erythrocyte SFAs were positively associated with childhood obesity risk, while n-3 PUFAs were inversely associated with childhood obesity risk.

\subsection{Effect of an Interdisciplinary Intervention on Health-Related Quality of Life: Secondary Analysis of the HealthTrack Study \\ E. P. Neale 1,2, L. C. Tapsell 1,2, A. Martin ${ }^{1,2}$, J. Russell ${ }^{3}$ and M. J. Batterham ${ }^{4}$ \\ 1 Faculty of Science, Medicine and Health, University of Wollongong, NSW, Australia \\ 2 Illawarra Health and Medical Research Institute, University of Wollongong, NSW, Australia \\ 3 Faculty of Social Sciences, University of Wollongong, NSW, Australia \\ 4 Faculty of Engineering and Information Sciences, University of Wollongong, NSW, Australia}


Exploring changes in health-related quality of life (HRQoL) measures is necessary when evaluating the effectiveness of health-care interventions. The aim of this analysis was to examine changes in HRQoL during the HealthTrack study, a 12-month randomised controlled trial comparing a novel interdisciplinary model of care for weight loss to usual care. Participants in the HealthTrack study were randomised to one of three study groups: control (usual care, $C$ ), interdisciplinary intervention $(I)$, or interdisciplinary intervention, plus a healthy food sample (30 g walnuts/day, IW). Participants completed the SF-12 at baseline, three, and 12 months, which was then converted to the SF-6D, a preference-based health state utility score. A SF-6D score of 0 indicates the poorest possible health state, whereas 1 indicates the best. Changes in SF-6D over time and between study groups were determined using a two-way mixed ANOVA. A total of $n=172$ participants had complete SF$6 \mathrm{D}$ score data for all three time points and were included in this analysis. Mean (standard deviation) baseline SF-6D scores were: C: 0.73 (0.12), I: $0.75(0.12), I W: 0.74(0.11)$. SF-6D scores were significantly higher in all groups at 12 months compared to baseline $(p=0.047)$. No significant group $(p=0.994)$ or interaction $(p=0.110)$ effect was found. Participating in a lifestyle intervention improved healthrelated quality of life regardless of group allocation.

\subsection{Effects of Whey Protein on the Suppression of Energy Intake, Gastric Emptying and Gut Hormone} Concentrations in Men and Women

C. Giezenaar ${ }^{1}$, N. Luscombe-Marsh ${ }^{2}$, A. Hutchison ${ }^{1}$, M. Horowitz ${ }^{1}$, I. Chapman ${ }^{1}$ and S. Soenen ${ }^{1}$

1 NHMRC Centre of Research Excellence in Translating Nutritional Science to Good Health, Discipline of Medicine, Royal Adelaide Hospital, The University of Adelaide, Australia

2 CSIRO, Australia

Protein-rich supplements are being used widely to lose weight. Particularly whey-protein, a fastacting protein rich in essential amino acids, is often used. Women, compared to men, appear to compensate less of a mixed-macronutrient preload in a subsequent meal, resulting in an increased total energy intake. This study aimed to determine the whey-protein loading effect on ad-libitum energy intake and underlying gastrointestinal mechanisms in healthy men and women. In randomised, double-blind order, eight women and eight men $\left(24 \pm 5 \mathrm{yrs}, 23 \pm 2 \mathrm{~kg} / \mathrm{m}^{2}\right)$ ingested a whey-protein drink $(\sim 450 \mathrm{~mL})$ containing $30 \mathrm{~g}(120 \mathrm{kcal})$ or $70 \mathrm{~g}(280 \mathrm{kcal})$ protein, or an iso-palatable control drink ( $\sim \mathrm{kcal})$ - followed by regular measurement of gastric emptying (3D-ultrasonography), blood glucose and plasma concentrations of insulin, glucagon, ghrelin, CCK, GIP and GLP-1 - and a buffet-style meal (180 min). Control day energy intake was lower in women than men $(791 \pm 87 \mathrm{kcal}$ vs. $1205 \pm 109 \mathrm{kcal}, p=0.010)$. Protein ingestion resulted in load-related effects on gastric emptying ( $p$ $<0.001)$, glucose, and gut hormones $(p<0.05)$. Women, compared to men, had slower gastric emptying $(p=0.021)$, lower concentrations of glucagon, CCK, GLP-1 and PYY and higher concentrations of glucose (all $p<0.05)$, and showed less suppression of energy intake by protein compared to control $(p=0.032)$. In women, total energy intake (drink + meal) after the 70 g-protein drink was higher compared to control $(p=0.033)$. In conclusion, the suppression of energy intake by whey-protein compared to control was less in healthy women than men, which was related to gastric emptying and postprandial gut hormone concentrations, and resulted in an increase in total energy intake in women.

\subsection{Analysis of the Metabolomic Responses to High Protein Meals in Women at Increased Metabolic Disease Risk}

Brenan Durainayagam 1, A. M. Milan 1, C. J. Mitchell 1, K. Fraser ${ }^{2}$, C. J. Henry ${ }^{3}$, M. C. Kruger 4 , N. C. Roy ${ }^{2}$ and D. Cameron-Smith ${ }^{1}$

1 Liggins Institute, University of Auckland, New Zealand

2 Food Nutrition \& Health, AgResearch, New Zealand

3 Clinical Nutrition Research Centre, Singapore Institute for Clinical Sciences, Singapore

4 Institute of Food Science and Technology, Massey University, New Zealand 
Metabolic syndrome (MetS) is a cluster of risk factors for diabetes and cardiovascular disease. Recently, metabolomics has been applied to gain insights into the complex metabolic changes associated with MetS. These analyses are commonly undertaken on blood from the fasted state, thus failing to capture dynamic metabolic responses to food. Current evidence suggests that a low glycaemic index (GI) and higher protein diet may improve metabolic control in MetS. This study aimed to comprehensively analyse the metabolic trajectories differentiating the dynamic responses of women with MetS from healthy women to high protein meals that included either low or high glycaemic index (GI) carbohydrates. Post-menopausal women (20 MetS, 20 healthy) consumed test meals on two separate mornings; the meals were high protein ( $30 \mathrm{~g}$ whey), containing either high or low GI carbohydrates. Fasted and postprandial (to $5 \mathrm{~h}$ ) blood samples were collected and analysed using non-targeted metabolomics by LC-MS. As expected, the fasting metabolic profiles of the MetS women differed from those of the healthy women; furthermore, the postprandial trajectories of many metabolites differed. A subset of metabolites, including uric acid and tryptophan, increased more post-ingestion in healthy women compared to the MetS women, independent of GI. Further, the GI of the meals impacted the postprandial dynamics of other metabolites. Carnitine, uridine, and threonine were more elevated with the lower GI meal, with the MetS and healthy women responding similarly. These analyses therefore distinguished the metabolic pathways that are altered or preserved in MetS, further elucidating the complexities of the metabolic dysfunction evident in women with compromised metabolic health.

\title{
2.125. Chrono-Nutrition - An Exploration of Individuals' Attitudes and Potential Barriers towards a Time- Restricted Feeding Pattern
}

\section{B. L. Devlin ${ }^{1}$, E. B. Parr ${ }^{1}$ and J. A. Hawley ${ }^{1,2}$}

1 Centre for Exercise and Nutrition, Mary MacKillop Institute for Health Research, Australian Catholic University, Melbourne, Australia

2 Sport and Exercise Sciences, Liverpool John Moores University, Liverpool, UK

Circadian disruption caused by irregular feeding patterns has adverse effects on health, independent of daily energy intake and diet composition. Chrono-nutrition, specifically timerestricted feeding (TRF), has been proposed as a powerful 'zeitgeber' for recalibrating the circadian clock. Manipulating the timing of food intake may be a simple, effective intervention to treat metabolic diseases. However, the acceptability of such a dietary pattern needs to be assessed. The aim of this study was to investigate the attitudes and potential barriers to a TRF dietary pattern. Participants followed an $8 \mathrm{~h}$ 'restricted' eating time frame of 1000 to 1800 and a $15 \mathrm{~h}$ 'unrestricted' eating time frame of 0700 to 2200 for five days each in a randomised order separated by a two week washout. In both conditions, participants consumed a high-fat diet (50\% total energy intake (TEI) fat, $30 \%$ TEI carbohydrate and 20\% TEI protein). Semi-structured individual interviews were conducted with 11 participants following to completion. The analysis revealed overall positive attitudes towards a TRF eating pattern. However, the main barriers to such a diet include a busy work schedule, family meal schedules, social event restrictions and hunger in the evening. Participants reported that TRF added structure to the day and stopped them snacking when not hungry. The findings demonstrate that TRF may be an acceptable and practical dietary approach. Therefore, research investigating both the potential health benefits and a mechanistic understanding of TRF is warranted.

\subsection{Cereal Fructan Extracts Modulate Intestinal Fermentation to Reduce Adiposity and Mineral} Excretion Compared to Oligofructose

\author{
D. P. Belobrajdic ${ }^{1}$, C. L. D. Jenkins ${ }^{2}$, Claus T. Christophersen ${ }^{3}$ and A. R. Bird ${ }^{1}$ \\ 1 CSIRO Health and Biosecurity, Adelaide, Australia \\ 2 Flinders University, Bedford Park, Australia \\ 3 Edith Cowan University, Perth, Australia
}

Intestinal fermentation of inulin-type fructans including oligofructose can modulate adiposity, improve energy regulation and mineral absorption. We aimed to determine whether cereal fructans 
had greater effects on impairing adiposity and mineral absorption compared to oligofructose. Thirtytwo male rats were randomly assigned to one of four dietary treatments that contained $0 \%$ fructan (control), or $5 \%$ fructan provided by either oligofructose, a barley grain fructan fraction (BGF) or a wheat stem fructan fraction (WSF). After one week on the diets, mineral absorption and retention were assessed. At four weeks, blood samples were collected for gut hormone analysis, adipose depots were removed and weighed, and the caecal digesta was analysed for $\mathrm{pH}$ and short chain fatty acids (SCFA). The BGF and WSF, but not oligofructose, resulted in lower total visceral fat weights than the control $(p<0.05)$. The fructan diets all raised caecal digesta weight, $\mathrm{pH}$ and total SCFA in comparison to the control. The caecal propionate levels for oligofructose were similar to the control and higher for BGF and WSF $(p<0.05)$. Plasma peptide YY and glucagon-like peptide-1 levels were elevated for all fructan groups when compared to control $(p<0.001)$ and gastric-inhibitory peptide was lower for the WSF compared to the other groups $(p<0.05)$. The fructan diets improved calcium and magnesium retention, which was highest for WSF $(p<0.05)$. BGF and WSF in comparison to oligofructose showed differential fermentation effects, altered gut hormone levels and reduced adiposity. Structural differences in fructan sources had favourable metabolic effects, suggesting greater improvements in energy regulation and mineral retention to those reported for oligofructose.

\title{
2.127. Fat Taste Sensitivity Is Associated with Short-Term and Habitual Fat Intake
}

\author{
A. Costanzo ${ }^{1}$, L. Orellana ${ }^{1}$, C. Nowson ${ }^{1}$, K. Duesing ${ }^{2}$ and R. Keast ${ }^{1}$ \\ 1 Deakin University, Geelong, VIC, Australia \\ 2 CSIRO Health \& Biosecurity, North Ryde, NSW, Australia
}

Evidence suggests individuals less sensitive to fat taste (high fat taste thresholds) may be overweight or obese, and consume greater amounts of dietary fat than more sensitive individuals (low fat taste thresholds). The aims of this study were to assess the associations between fat taste thresholds, anthropometric measurements, fat intake and liking of fatty foods in 68 Australian female twins (mean age 41.3 (15.6) (SD) years; mean body mass index (BMI) $26.3(5.7) \mathrm{kg} / \mathrm{m}^{2}$ ). Fat taste thresholds were assessed by a three-alternative forced choice methodology and transformed to an ordinal scale (FT rank); food likings were assessed through hedonic ratings of high-fat and reducedfat foods; and food intakes were assessed using a 24-h food recall and food frequency questionnaire. Linear mixed regression models were fitted, accounting for twin pairs as a random factor. FT rank was associated with dietary \% energy from fat (beta $=0.113$ [95\% CI: $0.006,0.219]$ ) and $\%$ energy from carbohydrates (beta $=-0.110[-0.189,-0.031]$ ), and the frequency of consumption of food items per day from the high-fat dairy (beta $=1.032[0.115,2.180]$ ), meat and meat alternatives (beta $=0.658[0.164$, $1.153]$ ), and grain and cereal (beta $=0.770[0.224,1.316]$ ) (adjusted for energy) food groups. There were no associations between FT rank and anthropometric measurements or hedonic ratings. Therefore, fat taste sensitivity appears to be associated with short-term fat intake, but not obesity in this group of females.

\subsection{Defining a Pathway for the Successful Implementation of a Healthy Food Policy in a University Environment}

\section{J. Dancey, C. Palermo, V. Ashton and H. Truby}

Monash University, Sydney NSW 2000, Australia

Lack of access to healthier drinks and snack foods in vending machines can contribute to poor food choices. Universities provide a powerful setting for healthier choices to be promoted, especially to young adults. The Victorian Government's Achievement Program encourages workplaces to implement the Healthy Choices Guidelines (HCG) in food retail, catering and vending using the traffic light classification, which stipulates that food and drinks are 50\% Green (best choice), 30\% Amber (choose carefully) and 20\% Red (limit). In 2017, Monash University commenced implementation of the Victorian Government's HCG, commencing with vending-potentially impacting 70,000 students and 7500 staff. Senior leadership were required to approve the alteration 
to the vending policy, which included a competitive tender process. The tender required compliance with the HCG plus standard financial and operational criteria. A photographic audit of 29 machines on one campus before the implementation of the HCG vending classified food and drinks as $64.8 \%$ Red, $13.7 \%$ Amber and $18.2 \%$ Green (3.3\% of product lines were empty). There are currently 35 new HCG-compliant vending machines on four campuses which offer $50 \%$ Green (an increase of $31.8 \%$ ), $30 \%$ Amber (an increase of $16.3 \%$ ) and $20 \%$ Red (a decrease of $44.8 \%$ ) drinks and snacks. This process highlighted the necessity for senior leadership support and for compliance with the HCG to be embedded into contractual arrangements. This has led to a large increase in the availability of Green drinks and snacks across a complex university environment. Implementation of the HCG will now extend to catering and retail within this setting.

\title{
2.129. Twenty-Four Hour Urinary Volume of Children: A Systematic Review of the Literature
}

\author{
K. Beckford ${ }^{1}$, C. A. Grimes ${ }^{1}$, C. Margerison ${ }^{1}$, L. J. Riddell ${ }^{1}$, S. A. Skeaff ${ }^{2}$ and C. A. Nowson ${ }^{1}$ \\ 1 Institute for Physical Activity and Nutrition Research, Deakin University, Australia \\ 2 Department of Human Nutrition, University of Otago, New Zealand
}

Urinary iodine excretion from 24-h urine samples (UIE, $\mu \mathrm{g} / 24-\mathrm{h}$ ) is considered to be the most accurate measure of daily iodine intake, although urinary iodine concentration (UIC) from spot urine samples are used to determine the iodine status of populations. UIC is assumed to be indicative of iodine intake based on the assumption of an average $1 \mathrm{~L}$ urine excretion in children in $24 \mathrm{~h}$, although this has never been verified. This study aimed to determine the average 24-h urinary output of children. A systematic literature search was conducted to identify studies which reported the mean 24-h urinary volume of children ( $>1 \mathrm{y}$ and $<19 \mathrm{y}$ ). Studies were categorised into two groups, younger (2-11 years old) and older children (12-19 years old). Thirty-seven studies were included, 12 of which reported results by gender. The overall mean (SD) urine volume reported was $728(251) \mathrm{mL} / 24-\mathrm{h}$. Older children had a significantly higher mean urine volume (973(195) vs. 650(214) mL/24-h, $p<$ 0.001). There was no difference in volume by gender. In conclusion, the average urinary output of children is less than $1 \mathrm{~L}$. Therefore, studies utilizing spot urine samples to classify the iodine intake of children where the mean urinary output is less than $1 \mathrm{~L}$ could contribute to an overestimation of the iodine intake of the population.

\subsection{A Case Study of Grandparents' Attitudes and Perceptions Regarding Healthy Lifestyle Behaviour in Their Young Grandchildren}

L. K. Bell ${ }^{1}$, S. Mortimer ${ }^{2}$, L. Matwiejczyk ${ }^{1}$, R. Perry ${ }^{4}$, I. Prichard ${ }^{3}$, C. Moores ${ }^{1}$, K. Mehta ${ }^{1}$ and M. Miller ${ }^{1}$

1 Nutrition and Dietetics, College of Nursing and Health Sciences, Flinders University, Adelaide 5000, Australia

2 Occupational Therapy, College of Nursing and Health Sciences Flinders University, Adelaide 5000, Australia

3 Health and Exercise Sciences, College of Nursing and Health Sciences, Flinders University, Adelaide 5000, Australia

4 Flinders Partners, Tonsley Precinct, Adelaide 5000, Australia

Grandparents can have a significant influence on young children's eating and activity behaviour, particularly if they are the primary carers of young children. This case study aimed to gain insight into the perspectives of custodial grandparents regarding the eating and activity behaviour of their young grandchildren. Semi-structured interviews (30-60 min) were conducted with custodial grandparents $(n=7)$, recruited through Grandparents for Grandchildren SA Inc. in Adelaide. Interview questions aimed to determine grandparents' beliefs, opinions, perceptions, knowledge, and support around the healthy eating and physical activity behaviour of their grandchild/ren aged 1-5 years. Interviews were coded in a four-phase process by three researchers who met frequently to discuss and agree on coding and emerging themes. Thematic analysis revealed four themes: (1) intergenerational differences in 'parenting' approach; (2) prioritisation of 
grandchildren's health and environment; (3) strong health awareness, beliefs and rules; and (4) high self-efficacy, low perceived requirement for support. These findings highlight that custodial grandparents adopt a different parenting approach the 'second time around' due to previous experience, have a perceived high level of knowledge, and a perceived high level of responsibility for their grandchild's wellbeing. Consequently, custodial grandparents are confident in their abilities and have strong beliefs and practices regarding the promotion of a positive nutrition and activity home environment. This case study suggests that support around young children's eating and activity behaviour is not desired, and perhaps not required, by this unique group of grandparents. This is a complex psycho-social topic that warrants further investigation in a larger cross-section of custodial grandparents.

\subsection{Predictors of Parental Unhealthy Food and Beverage Provision Using the Health Action Process Approach Framework}

\section{B. J. Johnson ${ }^{1}$, D. Zarnowiecki ${ }^{1}$, G. A. Hendrie ${ }^{2}$ and R. K. Golley ${ }^{1}$}

1 School of Pharmacy and Medical Sciences, University of South Australia, Adelaide 5000, Australia

2 Commonwealth Scientific Industrial Research Organisation Health and Biosecurity Flagship, Adelaide, 5000, Australia

Globally, children's intake of unhealthy foods and beverages is excessive. A key limitation of past research is the lack of a theoretical model to explain parent behaviour beyond the intention to limit the provision of unhealthy foods. This study aimed to utilise the Health Action Process Approach (HAPA) theoretical model to explain parents' intentions and behaviour in terms of limiting the provision of unhealthy foods and beverages to children within the home. Parents $(n=162)$ with children aged 4-7 years completed an online, self-reported questionnaire providing data on HAPA model constructs (risk perception, outcome expectancies, self-efficacy, intention, planning), child and parent demographics and potential confounders. The HAPA model includes the motivational phase, which leads to intention and the volitional phase that results in behaviour. Bivariate associations and multiple linear regression analyses, adjusting for predictors and confounders, were conducted. Model of fit statistics were appropriate in both models. In the motivational-phase-adjusted model, three risk perception variables, two outcome expectancy variables and action self-efficacy accounted for $36 \%$ of the variance in parents' intentions $(F(13,123)=5.21, p<0.001)$. In the volitional-phaseadjusted model, maintenance self-efficacy, two action planning variables, coping planning and three recovery self-efficacy variables explained $15 \%$ of the variance $(F(13,123)=1.70, p<0.068)$. The HAPA model captures several post-intentional factors and provides a framework to understand predictors of parental intentions and behaviour. Understanding these predictors may assist in designing interventions to support parents in executing and maintaining the intended food provision. Further research is needed to understand the complex interplay of factors that move parents from intentions to behaviour change.

\subsection{Reducing the FODMAP Content in the Breastfeeding Mother's Diet Alleviates the Symptoms of Infantile Colic: A Randomised, Controlled, Double-Blind, Crossover Study}

Iacovou, Marina, P. R. Gibson and J. G. Muir

Department of Gastroenterology, Central Clinical School, Monash University, Melbourne, Australia

Infantile colic, a common cause for presentation to emergency departments, lacks evidencebased therapies, yet 'windy-foods' are reduced by breastfeeding mothers. The study aimed to compare via a randomised, double-blinded, crossover study the effects of a maternal low-FODMAP diet (fermentable carbohydrates) and a typical Australian diet (control) in exclusively breastfeeding mothers on the crying-fussing duration of infants aged $\leq 9$ weeks with colic and address mechanisms. After a seven-day baseline period, mothers were fed a 10-day low-FODMAP or control diet and then crossed over to the alternate diet. All meals were provided. At baseline and on day 10 of each diet, mothers completed a depression, anxiety and stress scale (DASS), and collected samples of breast 
milk and infant faeces. Thirteen mother-infant dyads completed the study. Compared to the baseline crying-fussing durations (mean 269 [95\% CI: 224-314] $\mathrm{min} /$ day), there were greater reductions with the low-FODMAP diet $(152,102-210 \mathrm{~min} /$ day) when compared to the control diet $(185,135-234$ $\mathrm{min} /$ day; [ $p=0.05$; two-way-ANOVA]). This represented a reduction of 39 (75-3) min (8\% change) following the control-diet and 74 (107-40) $\mathrm{min}$ (35\% change) following the low-FODMAP diet $(p=$ 0.003). Lactose in the breast milk was stable and other dietary FODMAPs were not detected. Changes in infant faecal calprotectin and $\mathrm{pH}$ were similar with each diet. Although mean DASS scores were within the normal range, reductions in anxiety (0.9) and stress (9.4) compared to baseline $(3.5, p=$ $0.009 ; 13.4, p=0.004$, respectively; two-way-ANOVA) were seen with the control, but not the lowFODMAP diet. A maternal low-FODMAP diet was associated with a significant reduction in the crying-fussing duration of infants with colic. The mechanisms require elucidation.

\subsection{Development and Pilot Testing of a Nutrition Education Resource for Midwives}

C. Lucas ${ }^{1}$, K. E. Charlton ${ }^{1}$, A. T. McMahon ${ }^{1}$, L. Cummins ${ }^{2}$ and V. Elder ${ }^{2}$

1 School of Medicine, University of Wollongong, Wollongong NSW 2522, Australia

2 Illawarra Shoalhaven Local Health District, Wollongong NSW 2500, Australia

Good nutrition during pregnancy is essential for the optimal growth and development of the infant. Midwives are ideally placed to provide nutrition education to pregnant women, however a lack of nutrition training is a recognised barrier to the delivery of nutrition education by midwives. The aim of this study was to apply an evidence-based approach to develop a four-module (healthy eating, nutrition supplements, gestational weight gain and nutrition for breastfeeding) educational resource for midwives on nutrition. The resource was developed using the Australian Antenatal Care Clinical Guidelines as a framework for providing nutrition advice and was based on an experiential learning model. Teaching techniques included a problem-based learning approach using video simulations and opportunities for discussion, reflection and feedback. Modules were delivered during the lunch period at a public antenatal clinic over four consecutive weeks, followed by a block delivery so participants could catch up on missed content. Changes in knowledge and attitudes were assessed using pre-post self-completed surveys. A total of 26 midwives signed up for the programme, however only 14 were able to attend all four sessions. The key challenge in pilot testing the learning resource was the difficulty in engaging midwives within the clinical setting because of time constraints and a lack of predictability in daily schedules. The application of adult learning theory was valuable in designing nutrition education materials for midwives and the resulting resource was perceived to be useful for improving knowledge. Future projects should consider more flexible delivery options to allow for the unpredictability of midwifery work.

\subsection{Are Paediatric Reference Intervals for Lipid Profiles Useful for Dietary Intervention Trials?}

K. Deering, A. Nicholl, A. Devine, P. Lyons-Wall, C. Christophersen, M. Boyce, T. Mori, D. Lawrence and T. O'Sullivan

School of Medicine and Pharmacology Royal Perth Hospital Unit, The University of Western Australia, 6907, Australia

Lipid profiles in children are generally compared to extrapolated reference intervals that theoretically represent $95 \%$ of the reference population. However, these lack local and national agreement in Australia and often cover a wide age range. The aim of this study was to compare child lipid profiles in a small population of West Australian children with central reference intervals. Sixteen healthy 4-6-year-old children ( $\mathrm{n}=$ eight female) provided fasting blood for the analysis of lipid profiles as part of a larger dairy intervention study. Total cholesterol, LDL-cholesterol (LDL-C), HDL cholesterol (HDL-C) and triglycerides were measured at a NATA-accredited laboratory using an Abbott autoanalyser. Interim data analysis found all the values for triglycerides and HDL-C were within the central reference intervals. Almost half $(43 \%)$ of the $4-6$ year olds had elevated total cholesterol levels (4.3 IQR 3.8-4.7 mmol/L) and 31\% had elevated LDL-C (2.6, IQR 1.9-2.9 mmol/L) 
compared to reference intervals (total cholesterol: $2.8-4.5 \mathrm{mmol} / \mathrm{L} ; \mathrm{LDL}-\mathrm{C}<2.9 \mathrm{mmol} / \mathrm{L}$ ). Of those with high total cholesterol $(n=7)$, five had high LDL-C levels. Lipid concentrations may be raised in early childhood due to increased physiological need, but are not reflected in current reference intervals. This may be due to the lack of age specificity in the reference intervals. Our results align with recent findings from a larger longitudinal Australian paediatric cohort of 8-12 year olds. Current reference intervals may not be appropriate for younger children.

\subsection{A Dietary Guidelines Index Is a Valid Measure of Diet Quality in a Cohort Study from Childhood to Adulthood \\ J. E. Wilson 1, S. L. Blizzard, L 1, Gall 1, C. G. Magnussen 1, T. Dwyer ${ }^{2}$, A. Venn ${ }^{1}$ and K. J. Smith ${ }^{1}$ \\ 1 Menzies Institute for Medical Research, University of Tasmania, Australia, 7005. \\ 2 The George Institute for Global Health, University of New South Wales, Sydeney, Australia, 2042.}

To our knowledge, no previous studies have validated a dietary index as a measure of diet quality from childhood to adulthood. This study aimed to validate a Dietary Guideline Index (DGI), with a consistent scoring approach for both children/adolescents and adults, as a measure of diet quality in a cohort study. The DGI was based on the 2013 Australian Dietary Guidelines and included nine core components with two additional components for adults. DGI scores were calculated from the Childhood Determinants of Adult Health study, which included a 24-h food record from children in 1985 ( $n=5208$, age: $10-15$ years, $50.3 \%$ male), and a 127-item food frequency questionnaire in 20042006 ( $n=1849$, age: $28-36$ years, $46.6 \%$ male). Construct and criterion validity were examined by assessing cross-sectional associations with nutrient intake, sociodemographic, lifestyle, and cardiometabolic variables, using linear regression. In childhood, lower DGI scores were significantly $(p<$ 0.05) associated with smoking, obesity (males only), and lower area-level socioeconomic status, parental education, academic achievement, and self-reported health. Scores were inversely associated with sugar and fat intakes, and positively associated with fibre, protein and micronutrient intakes. In adulthood, lower DGI scores were associated with having children, smoking, lower education, occupational status, and self-reported health, higher total and LDL cholesterol, higher BMI (men only), and insulin insensitivity (women only). The index reflects nutritional intake and discriminates between characteristics with associations in expected directions. The validation of the index in a cohort study provides a basis upon which to investigate diet quality and associations with health outcomes from childhood.

\subsection{The Digital Education to Limit Salt in the Home (DELISH) Program: Outcome Evaluation among Children}

\section{A. Grimes, A. Booth, D. Khokhar, M. West, C. Margerison, K. J. Campbell and C. A. Nowson}

Institute for Physical Activity and Nutrition, School of Exercise and Nutrition Sciences, Deakin University, Victoria, Australia

Australian schoolchildren eat too much salt. Online technology presents an opportunity to provide families with educational messages related to salt intake and healthy eating. The aim of this study was to determine if participation in the five-week web-based DELISH program reduced salt intake and improved salt-related knowledge, attitudes and behaviours (KABs) and self-efficacy among schoolchildren. Child/parent dyads $(n=102)$ were recruited from five Victorian government schools. Children completed pre-test and post-test measures: (i) 24-h urine collection to assess salt intake; and (ii) an online survey to assess KABs and self-efficacy. The appropriate paired $t$-test or Wilcoxon signed rank test was used to assess change in pre- and post-test measures. Intervention delivery was assessed via online session log-in rates. The intervention was delivered to 83 children (mean age 9.2 (SD 0.8) years, 59\% girls) and between 35\% and 76\% of children viewed each weekly session. Among the 51 children who provided a valid pre- and post-test 24 -h urine collection, there was no change in salt intake ( $5.4 \pm$ SE $0.4 \mathrm{~g} / \mathrm{d}$ vs. $5.3 \pm 0.3 \mathrm{~g} / \mathrm{d}, p=0.75)$. Among the 75 children who completed a pre- and post-test survey there was a significant improvement in scores for salt-related 
knowledge ( $14 \pm 0.3$ vs. $17 \pm 0.3, p<0.001$ ), behaviour (median 5(IQR 2 ) vs. $7(1), p<0.001$, indicating improved discretionary salt use habits) and self-efficacy (median 4(IQR 2) vs. 5(2), $p<0.001$ ), but no change in attitude score. In conclusion, participation in the DELISH program did not change children's salt intake but did favorably alter their salt-related knowledge, behaviour and self-efficacy.

\subsection{An Umbrella Review of the Effectiveness of Strategies to Strengthen Healthy Eating Practices within} Early Childhood Settings

L. Matwiejczyk ${ }^{1}$, K. Mehta ${ }^{1}$, J. Scott ${ }^{2}$ and J. Coveney ${ }^{3}$

1 Discipline of Nutrition and Dietetics, College of Nursing and Health Sciences, Flinders University, Adelaide, South Australia 5042, Australia

2 School of Public Health, Faculty of Health Sciences, Curtin University, Western Australia 6102, Australia

3 Discipline of Public Health, College of Medicine 7 Public Health, Flinders University, South Australia 5042, Australia

Where once the family home was the main influence on developing children's food preferences and dietary food patterns, early childhood education and care (ECEC) educator practices and the childcare food environment also have a crucial role in influencing food choices in young children aged two to five years when food preferences are being developed; these will track into later life. This manuscript describes a systematic review of several recent existing reviews to provide an overall examination of the effectiveness of strategies and practices to improve the implementation of healthy eating policies and practices within ECEC settings. METHOD: Eight databases were searched for systematic reviews published until May 2017 from high-income or similar countries. Reviews were critically appraised using the JBI critical appraisal instrument for systematic reviews and research synthesis. To avoid the risk of bias, the JBI data extraction tool for systematic reviews and research synthesis was also used. RESULTS: Ten reviews were selected. Children's dietary food patterns were significantly influenced by the strategies and practices implemented in ECEC settings. Educators positively promote healthy eating behaviours in children. Primary prevention obesity interventions significantly influence children's dietary food patterns, but not children's weight, unless parents are involved. Interestingly, the interventions implemented within a research context are mostly effective, but not if implemented without this support. CONCLUSION: Educators' practices, healthy eating policies and the ECEC food environment positively influence children's dietary food patterns. Practices and strategies can be further strengthened with parental involvement. The translation of research interventions and evidence-based recommendations into practice by educators also warrants further exploration.

\subsection{Data Quality Management in Clinical Trials}

L. Houston ${ }^{1,2}$, Y. Probst ${ }^{1,2}$, Y. Ping ${ }^{3}$ and A. Martin ${ }^{1,2}$

1 School of Medicine, Faculty of Science, Medicine and Health, University of Wollongong, Wollongong, NSW 2522, Australia

2 Illawarra Health and Medical research institute, University of Wollongong, Wollongong, NSW 2522, Australia

3 School of Computing and Information Technology, Faculty of Engineering and Information Sciences, University of Wollongong, Wollongong, NSW 2522, Australia

The life sciences have been transformed through scientific breakthroughs that have improved medical knowledge and the patient care of those suffering from chronic diseases. This process collects vast amounts of data from clinical trials. Strategies and interventions are implemented to avoid scientific mistakes, reduce error and assure the accuracy of findings. Little is known about the methods used to assess data quality management and its level of rigor in clinical trials. This feasibility study aims to explore current data quality management procedures in clinical trials. Relevant information about data quality management methods/procedures, error levels, data monitoring, staff training and development were collected. Staff members from 142 clinical trials listed on the National Health and Medical Research Council (NHMRC) clinical trials website were invited to complete a 
short, self-reported, semi-quantitative, anonymous online survey. Those from $20(14 \%)$ trials completed the survey. Centralised monitoring $(65 \%)$ was the most common procedure to ensure high-quality data. Ten $(50 \%)$ trials reported having a data management plan and two trial sites utilised an error acceptance level to minimise discrepancies, $<5 \%$ and $5-10 \%$, respectively. The quantity of data variables checked (10-100\%), frequency of visits (once-a-month to annually), and types of variables $(100 \%$, critical data or critical and non-critical data) for data monitoring differed between the trials. The average time spent on staff training per person over a 12-month period was $11.58 \mathrm{~h}$ and the type of training varied. It appears that ad-hoc methods are implemented pragmatically to ensure data quality. The study confirms the necessity to further investigate the "best methods" for data quality management in clinical trials.

\subsection{Effects of Fruit and Vegetable Consumption on Immunity and Inflammation-A Systematic Literature} Review and Meta-Analysis

B. Hosseini ${ }^{1}$, B. S. Berthon ${ }^{1}$, A. Saedisomeolia ${ }^{2,3}$, M. Starkey ${ }^{1}$, A. Collison ${ }^{1}$, P. A. B. Wark ${ }^{1}$ and

L. G. Wood ${ }^{1}$

1 Centre for Healthy Lungs, Hunter Medical Research Institute, University of Newcastle, Newcastle, NSW 2308, Australia

2 School of Nutrition Sciences and Dietetics, Tehran University of Medical Sciences, Tehran, Iran

3 School of Medicine, Western Sydney University, Sydney, NSW 2751, Australia

Inflammation is associated with an increased risk of a range of chronic diseases. A diet high in fruit and vegetables may help to reduce inflammation, as fruit and vegetables are a rich source of antioxidants and other biologically active substances that may improve immune function. The aim of this systematic literature review was to investigate the effects of fruit and/or vegetable intake on immunity (including immune responses to virus infection, and inflammation) in different diseases and conditions. Electronic databases including PubMed, Cochrane, CINAHL, and EMBASE were searched up to February 2017. A total of $n=74$ studies met the inclusion criteria. An experimental design was most commonly used $(n=64)$ with $n=8$ cross sectional studies and $n=2$ cohort studies. Amongst the observational research, $\mathrm{n}=8$ studies found an inverse association between intakes of fruit and/or vegetables and inflammatory biomarker(s). Similarly, most of the intervention studies $(73 \%, n=47)$ reported beneficial effects of fruit and/or vegetable intake on at least one systemic or airway inflammatory biomarker, while $\mathrm{n}=17$ studies found no significant relationship. A metaanalysis of the included studies showed that fruit and/or vegetable intake increased the $\gamma \delta$-T cell population $(p<0.05)$, which is involved in protecting the host against pathogens as well as preventing chronic inflammation. In conclusion, this review suggests that higher fruit and vegetable consumption leads to both a reduction in pro-inflammatory mediators and an enhanced immune cell profile.

\subsection{Homocysteine Is Associated with Alzheimer's Disease but Not as Strongly as APOE4: A Case- Control Study}

N. D'Cunha, E. Georgousopoulou, L. Boyd, J. Sturm, M. Lucock, A. McKune, J. Thomas, D. Mellor, P. Roach and N. Naumovski

Faculty of Health, University of Canberra, Canberra 2617, Australia

The plasma homocysteine (Hcy) concentration is influenced by dietary B-vitamin intake and elevated Hcy levels are associated with a greater risk of developing Alzheimer's disease (AD). The Apolipoprotein E- $€ 4$ (APOE4) isoform has also been found to strongly increase the risk of developing AD. However, there is conflicting evidence about its link to Hcy. This retrospective case-control study of 126 age-matched elderly Australians (65-83 years), compared 63 clinically-diagnosed AD sufferers ( 35 females $(\mathrm{F})$ and 28 males $(\mathrm{M})$, age $=77.1 \pm 5.3$ years) with 63 healthy controls $(\mathrm{HC})(38 \mathrm{~F}$ and $25 \mathrm{M}$, age $=76.9 \pm 4.7$ years). The plasma concentrations of Hcy and APOE4 were determined using high pressure liquid chromatography and an enzyme-linked immunosorbent assay kit, 
respectively. The Hcy levels in the AD group were significantly elevated (AD mean $=11.61 \pm 4.9$ $\mathrm{mol} / \mathrm{L}$ ) compared with the HC group (HC mean $=9.39 \pm 2.6 \mathrm{~mol} / \mathrm{L} ; p=0.028)$. The prevalence of the APOE4 isoform in the plasma of the AD group was double $(37 / 63,59 \%)$ that of the HC group (18/63, $29 \%)$. Following adjustment for age and gender, logistic regression analysis revealed a significant independent relationship between Hcy and $\mathrm{AD}$ (Odd's ratio $(\mathrm{OR})=1.18,95 \%$ confidence interval $(\mathrm{CI})$ 1.04-1.34, $p=0.01)$. However, in the same model, the independent relationship between the presence of the APOE4 isoform and AD was much stronger $(\mathrm{OR}=3.69,95 \% \mathrm{CI}=1.66-8.20, p=0.001)$. This suggests that although there is an independent association between an elevated Hcy and AD, this is not as strong as the association between the APOE4 phenotype and AD. Further investigation is required to understand the interaction between dietary B-vitamins, Hcy and APOE4 in AD prevention.

\subsection{Can a Modified Mediterranean Diet Reduce Inflammation in Men with Prostate Cancer?}

\section{S. Erdrich}

Affiliation not provided.

Prostate cancer is the most commonly diagnosed malignancy and the third leading cause of mortality in New Zealand men. Global distribution patterns of PCa suggest a link to diet and lifestyle.

Objectives: The goal of this study was to determine the effect of following a Mediterranean-style diet on inflammation in men with prostate cancer.

Methods: Men with low-grade prostate cancer, $(n=20)$, aged 52-74, followed a modified Mediterranean diet for three months. Health-related information, four-day diet records and blood samples were collected at baseline. At study end, blood samples and dietary data were assessed. Analysis of PSA, C-reactive protein, whole-blood fatty acids, and DNA damage were performed. Evaluation of the interactions of the diet with these biomarkers was undertaken.

Results: There were no changes in the C-reactive protein or PSA levels. Dietary adherence scores increased from 6.3 to 13.6 out of $20(p<0.0001)$ and were inversely correlated with DNA damage $(p=0.013)$, as were whole blood monounsaturated fatty acids $(p=0.009)$, dietary intake of legumes $(p=0.004)$, folate $(p=0.023)$, green tea $(p=0.002)$ and vitamin $C(p=0.007)$. DNA damage was positively correlated with the intake of dairy products $(p=0.008)$, red meat $(p=0.007)$, whole blood n6FA $(p=0.015)$ and with the $\mathrm{n} 6: \mathrm{n} 3$ ratio $(p=0.023)$.

Conclusion: We have shown, for the first time (to the best of our knowledge), that a high polyphenol diet, modelled on Mediterranean traditions, results in a significant reduction in DNA damage in New Zealand men with low-grade prostate cancer.

\subsection{Association between Lipid Metabolism in Decidual and Villous Tissues of Placenta and Early Spontaneous Abortions in Chinese}

Kelei Li ${ }^{1}$, Xiaotian Zhang ${ }^{2,3}$, Xiaoying Zheng ${ }^{3, *}$ and Duo $\mathrm{Li}^{1,4, *}$

1 Institute of Nutrition and Health, Qingdao University, Qingdao, China

2 The Key Laboratory of Cell Proliferation and Regulation Biology of Ministry of Education, Institute of Cell Biology, College of Life Sciences, Beijing Normal University, Beijing, China

3 Institute of Population Research/WHO Collaborating Center on Reproductive Health and Population Science, Peking University, Beijing, China

4 Department of Food Science and Nutrition, Zhejiang University, Hangzhou, China

* Correspondence: xzheng@pku.edu.cn (X.Z.); duoli@zju.edu.cn (D.L.)

Background: The aim of the present study is to assess the association between lipid metabolism in the decidual and villous tissue of placenta and spontaneous early pregnancy loss.

Methods: Seventy women with early spontaneous abortion and 29 healthy pregnant women who asked for an induced abortion were included in the case and control, respectively. Decidual and villous tissues of the placenta were collected after termination of the pregnancy. Fatty acids were detected by the standard method. Metabonomics analysis was conducted by UPLC-QTOFMS. 
Results: The gestational age of all subjects ranged from 4 to 10 weeks. The C18:3n-3 in decidual and villous tissues, total $n-3$ polyunsaturated fatty acid (n-3 PUFA) in decidual tissue, and C18:2n-6 in villous tissue were all significantly lower in the women in the case group than in healthy pregnant women. These results indicated that a lack of those PUFA mentioned above is associated with the occurance of early spontaneous abortion. The ratio of C20:4n-6/C20:5n-3 in the villous tissue was significantly higher, but prostaglandin $\mathrm{I}_{2}\left(\mathrm{PGI}_{2}\right)$ as well as hydroxyeicosapentaenoic acid (HEPE), Leukotriene $\mathrm{B}_{5}\left(\mathrm{LTB}_{5}\right)$ and thromboxane $\mathrm{B}_{3}\left(\mathrm{TXB}_{3}\right)$ in the villous tissue were significantly lower in women with early spontaneous abortion than in healthy ones. Moreover, low lysophosphatide levels in the decidual and villous tissues and the low content of diacylglycerol in the villous tissue were also associated with the occurance of early spontaneous abortion.

Conclusion: A lack of essential fatty acids and n-3 PUFA, high ratio of C20:4n-6/C20:5n-3, abnormal eicosanoids metabolism and low content of lysophosphatide and diacylglycerol in the placenta were potential risk factors for early spontaneous pregnancy abortion in Chinese women.

\title{
2.143. Effects of Pectin and Mango Pulp on Triglycerides, Cholesterol and Bile Acids in Pigs as a Model for
} Humans

P. Gunness ${ }^{1}$, H. Zhai ${ }^{1,2}$, B. A. Williams ${ }^{1}$, A. Lisle ${ }^{3}$ and M. J. Gidley ${ }^{1}$

1 ARC Centre of Excellence in Plant Cell Walls, Queensland Alliance for Agriculture and Food Innovation, The University of Queensland, St Lucia QLD 4072, Australia

2 South China Sea Fisheries Research Institute, Chinese Academy of Fishery Sciences, Guangzhou 510300, China

3 School of Agriculture and Food Sciences, Faculty of Science, The University of Queensland, St Lucia QLD 4072, Australia

(1) Background: There is some evidence from intervention studies that pectin, a soluble dietary fibre, could lower the risk for cardio-vascular disease (CVD) by improving plasma cholesterol. However, the mechanisms of action remain unclear, although an excess excretion of bile acids (BAs) in the faeces has been postulated. (2) Methods: Pigs were fed a control diet ( $\mathrm{n}=10)$, or diets containing $10 \%$ apple pectin extract $(n=10)$ or $15 \%$ mango pulp $(n=10)$ for four weeks. Jugular blood and intestinal contents from the proximal and distal jejunum and terminal ileum were collected. (3) Results: Compared to the control, diets containing pectin or mango pulp significantly decreased the plasma total cholesterol (TC) by $18 \%(p<0.01)$ and $9 \%(p<0.05)$, respectively. Pectin also decreased the LDL-C and triglycerides (TG) by $17 \%(p<0.05)$ and $28 \%(p<0.01)$, respectively, while the mango pulp caused an increase of $31 \%(p<0.01)$ in TG. The total bile acid (TBA) concentrations significantly increased by 1 -fold $(p<0.01)$ in the blood and by 2.5 -fold $(p<0.01)$ in the jejunum and 3-fold $(p<0.01)$ in the terminal ileum with the pectin, but not the mango diet. (4) Conclusions: The combined blood and upper digestive tract results suggest that the mechanism(s) by which pectin causes blood TC and TG reduction may differ for soluble and particulate forms and further studies are warranted.

\subsection{The Level of Serum 25(OH)D $D_{3}$ and Its Influence Factors in the Rural Population of Henan} Province in China

\author{
F. Yu, X. Li, H. L. Jiang, S. C. Yu, Y. Wang, C. J. Wang, Y. Ba and W. J. Li * \\ College of Public Health, Zhengzhou University, Zhengzhou 450001, China \\ * Corresponding Author
}

Background: Many studies suppose that vitamin D plays an important role in the process of type 2 diabetes mellitus (T2DM). People in the most populous province of Henan in China are suffering from T2DM. Thus, the purpose of this study was to explore the level of serum $25(\mathrm{OH}) \mathrm{D}_{3}$ and its influence factors in the rural population of Henan province.

Method: A total of 2215 subjects were recruited to study vitamin D status. The basic information, anthropometry and biochemical indexes were conducted. The categorical data were analyzed using the chi-square test. The continuous variables were compared using the student's 
test, one-way analysis of variance (ANOVA), and multiple factorial analysis of variance or nonparameters tests. All analyses were performed using SPSS software (Version 21.0).

Results: The results showed that the average serum $25(\mathrm{OH}) \mathrm{D}_{3}$ level was $27.51 \pm 22.33 \mathrm{ng} / \mathrm{mL}$. The percentage of vitamin D deficiency and insufficiency was nearly $77.9 \%$. The $25(\mathrm{OH}) \mathrm{D}_{3}$ levels in male and those aged below 55 were greater than in females and those aged above $55(p=0.049$ and 0.001). Those with abnormal WHR, WHtR, serum TG and HDL-C have lower 25(OHD)s levels $(p<$ 0.05). The $25(\mathrm{OH}) \mathrm{D}_{3}$ levels in $\mathrm{T} 2 \mathrm{DM}$ or hypertensive patients were significantly lower than those in healthy people (p0.02).

Conclusion: The prevalence of vitamin D deficiency and insufficiency in the investigation areas is high, and age, obesity and lipid metabolic index may influence the $25(\mathrm{OH}) \mathrm{D}_{3}$ level negatively. Therefore, rational measures should be established to improve the vitamin D status in these areas.

Keywords: vitamin D status survey; 25(OH)D3 level; influence factors

\title{
2.145. Examination of Risk Factors Related to Stress Fractures in Female, Long-Distance Runners
}

A. Minematsu ${ }^{1}$, K. Yamamoto ${ }^{1}$, M. Kieko ${ }^{2}$, M. Sekine ${ }^{2}$, K. Kakeru ${ }^{3}$, M. Yuichi ${ }^{1}$, T. Yano ${ }^{1}$ and M. Ota ${ }^{1}$

1 Graduate school of Food and Nutrition Science, Toyo University, Gunma, Japan

2 Graduate school of Life Science, Toyo University, Gunma, Japan

3 Research Institute of Life Innovation, Toyo University, Gunma, Japan

Stress fractures occur most commonly in female, long-distance runners. They are currently difficult to predict and prevent. Bone density and bone quality are determinants of bone strength, and collagen cross-linking is a determinant of bone quality. For cross-link formation, pentosidine, homocysteine, and single nucleotide polymorphism (SNP) in the methylenetetrahydrofolate reductase (MTHFR), which is a homocysteine metabolism-related enzyme, are related. This study investigated the contribution of bone-related factors using principal component analysis. Overall, seven female, long-distance runners participated in this study. The following risk factors were evaluated: SNP in the MTHFR, plasma pentosidine and homocysteine levels, age, athletic career, running distance, menstrual cycles, past stress fracture, and bone mineral density at each site. Principal component analysis was used for the statistical analysis. The factor with largest contribution to the stress fracture risk within the first principal component was SNP in the MTHFR. There was no significant difference in BMD at various sites in the SNP type. Hetero- and homozygous had higher pentosidine levels than wild type ( $p$ for trend $=0.001$ ). Similarly, past stress fracture experience also showed a significant tendency ( $p$ for trend $=0.001$ ). SNP in the MTHFR is a risk factor for stress fractures. It is necessary to study multiple factors rather than a single factor.

\subsection{The Role of Dairy in the Management of Muscle Mass and Function in People aged 50+ yrs: A Systematic Review and Meta-Analysis}

\author{
T. Wycherley ${ }^{1}$, M. Riley ${ }^{2}$, W. Stonehouse ${ }^{2}$, P. Taylor ${ }^{2}$, G. Brinkworth ${ }^{2}$ and N. Luscombe-Marsh ${ }^{2}$ \\ 1 University of South Australia, Adelaide, SA, Australia \\ 2 Commonwealth Scientific and Industrial Research Organisation, Adelaide, SA, Australia
}

A meta-analysis of randomised controlled trials (RCTs) was performed to investigate the effects of whole dairy foods and supplements containing dairy ingredients, with and without resistance training (RT), on lean mass, strength, muscle size, muscle protein synthesis and physical function in people aged 50+ years. RCTs published prior to 1 March 2015 were identified by a comprehensive systematic search of scientific databases. Eligible reports were critically appraised in duplicate. Data were pooled using random-effect meta-analyses. Thirty-four RCTs were eligible for inclusion. Dairy consumed in combination with RT resulted in a small increase in lean mass (weighted mean difference [95\% CI]; $0.5[0.02,0.98] \mathrm{kg} ; p=0.04 ; \mathrm{I}=68 \%)$ but had no effect on muscle size $(p=0.80)$, leg press strength $(p=0.40)$ or grip strength $(p=0.29)$. Dairy consumed in the absence of RT had no effect on lean mass $(p=0.58)$. There were insufficient data to analyse gait speed. Dairy protein supplements 
of different dosages (i.e., 20 vs. $40 \mathrm{~g} /$ day) increased the fractional synthetic rate of myofibrilliar protein both in the context of RT $(0.03[0.0,0.06] \% / \mathrm{hr} ; p=0.02 ; \mathrm{I}=87 \%)$ and without RT $(0.02[0.01$, $0.02] \% / \mathrm{hr} ; p<0.001 ; \mathrm{I}=0 \%$ ). In conclusion, increased dairy intake (at least $20 \mathrm{~g}$ per day, particularly in the form of dairy protein supplements) when combined with RT, is an effective nutritional strategy to augment lean body mass in people aged $50+$ years. While dairy protein acutely up-regulates muscle protein synthesis in this age group irrespective of $\mathrm{RT}$, this effect does not directly translate into increases in regional muscle size, muscle strength or function.

\subsection{Dairy Supplementation Reduces Bone Resorption and Improves IGF-1 and Slows Bone Loss in Institutionalised Elderly: A Cluster Randomised Study}

S. Iuliano ${ }^{1}$, S. Poon ${ }^{1}$, J. Robbins ${ }^{1}$, A. Sones ${ }^{2}$, X. Wang ${ }^{1}$ and E. Seeman ${ }^{1}$

1 Department of Endocrinology, University of Melbourne, Austin Health, Heidelberg 3084, Australia

2 Austin Health, Heidelberg 3084, Australia

Fracture rates are highest in institutionalised elderly people with a low calcium intake. Calcium supplementation is purportedly associated with adverse cardiac outcomes, while dairy sources of calcium are not. We aimed to determine whether increasing dairy intake improved bone metabolism and slowed bone loss in aged-care residents. Fifty-six facilities were randomised to high-dairy ( $\mathrm{n}=$ 26) or usual menu (controls, $\mathrm{n}=30$ ). For 215 residents (mean age $86 \pm 8$ years, $65 \%$ females), food consumption was measured using plate waste analysis, and fasting morning blood was analysed for bone markers (CTX, P1NP), PTH, and IGF-1 at baseline and three months. Volumetric bone mineral density (vBMD) was measured at the distal tibia and radius using high-resolution-pQCT at baseline and 12 months. Group differences were determined using robust regression. At baseline, no group differences were observed; mean calcium $(687 \pm 261 \mathrm{mg} / \mathrm{d})$ and protein $(0.8 \pm 0.3 \mathrm{~g} / \mathrm{kgBW})$ intakes were below recommended levels. Dairy supplementation augmented dairy $(1.7 \pm 1.1$ serve/d, $p<$ $0.001)$, calcium $(470 \pm 339 \mathrm{mg} / \mathrm{d}, p<0.001)$ and protein $11 \pm 20 \mathrm{~g} / \mathrm{d}(p<0.001)$ intakes. CTX declined $(414 \pm 250$ vs. $375 \pm 266 \mathrm{ng} / \mathrm{L}, p=0.034)$ with intervention but remained unchanged ( $423 \pm 230 \mathrm{vs.} 424$ $\pm 259 \mathrm{ng} / \mathrm{L}, p=0.981$ ) in controls. P1NP remained unchanged with intervention ( $54 \pm 38$ vs. $50 \pm 38$ $\mathrm{mcg} / \mathrm{L}, p=0.116$ ) but declined in controls ( $54 \pm 34$ vs. $46 \pm 26 \mathrm{mcg} / \mathrm{L}, p=0.006$ ). IGF-1 differed between groups by month three $(p=0.011)$. No changes were observed for PTH. vBMD at the tibia remained unchanged with intervention $\left(212 \pm 48 \mathrm{v} 212 \pm 49 \mathrm{mgHA} / \mathrm{cm}^{3}\right)$, but decreased in controls $(243 \pm 90 \mathrm{v}$ $\left.240 \pm 91 \mathrm{mgHA} / \mathrm{cm}^{3}\right)$, in whom porosity increased. Improving calcium intake using dairy foods reduced markers of resorption and improved IGF-1, and abated bone loss, which may reduce fracture risk in aged-care residents.

2.148. Dietary Patterns and Relationships with Depressive Symptoms and with Cognitive Impairment in a Cohort of Older Adults

\section{T. L. Spriggs, K. M. Dickinson, Yaxley, A and Miller, M}

Nutrition and Dietetics, School of Health Sciences, Faculty of Medicine, Nursing and Health Science, Flinders University, Bedford Park, SA 5042, Australia

Evidence suggests that dietary patterns may influence the development of depressive symptoms and cognitive impairment, however, there are few longitudinal studies that have investigated these relationships in older adults. This study aimed to explore the associations between dietary patterns and depressive symptoms or cognitive impairment by secondary analysis of the Australian Longitudinal Study of Ageing, a cohort study of South Australians aged $\geq 65$ years. Baseline data from a validated 170-item food frequency questionnaire (FFQ) was used to derive dominant dietary patterns using factor analysis. Logistic regression was used to determine whether there were any associations between the dietary patterns and depressive symptoms and cognitive impairment with data available at baseline and 11 years follow-up. In $n=482$ participants, five dominant dietary patterns were identified and labelled accordingly. The dietary patterns 'vegetables and fruit' (tertile 2 (T2): OR 0.31, 95\% CI 0.11, 0.89) and 'grains, dairy and meat alternatives' (T3: OR 0.28, 95\% CI 0.11, 
0.73) showed a significant inverse association with cognitive impairment. A significant inverse association was found between $\mathrm{T} 2$ of the 'high fat and salt foods and offal meat' dietary pattern and depressive symptoms (OR 0.26, 95\% CI 0.08, 0.86), and cognitive impairment (OR 0.32, 95\% CI 0.12, 0.86). These results suggest that the identified dietary patterns may decrease the risk of cognitive impairment and of depressive symptoms in older adults, however further investigation of dietary patterns is needed to understand these relationships.

\subsection{Assessing the Relationship between Diet and Lung Function in Middle-Aged Australians Using Nutrient-Based Dietary Patterns}

S. Byrne ${ }^{1}$, E. Milanzi ${ }^{1}$, J. A. Burgess ${ }^{1}$, M. J. Abramson ${ }^{2}$, E. H. Walters ${ }^{1}$, D. Johns ${ }^{3}$, M. Jenkins ${ }^{1}$ and A. Hodge ${ }^{1,4}$

1 Centre for Epidemiology and Biostatistics, The University of Melbourne, VIC, Australia

2 School of Public Health \& Preventive Medicine, Monash University, VIC, Australia

3 School of Medicine, University of Tasmania, TAS, Australia

4 Cancer Council Victoria, VIC, Australia

Despite the strong correlation between nutrient intakes and various health outcomes, there has been no research exploring the relationship between nutrient-based dietary patterns and lung function. We examined this relationship in a population-based sample of middle-aged Australians. Participants underwent spirometry and answered a food frequency questionnaire $(n=1193)$. Energyadjusted nutrient intake was included in a principal components analysis. Scores were calculated for eight components explaining $82 \%$ of the variation in nutrient intake and their relationship with lung function was assessed using linear regression. Age, gender, height, energy intake, BMI, asthma, atopy and smoking were included as confounders in all regression models. Component 1 , representing a diet high in potassium, magnesium, folate and fibre, was positively associated with forced expired volume in one second $\left(\mathrm{FEV}_{1}\right)(p$-value $=0.002)$ and forced vital capacity $(\mathrm{FVC})(p<0.001)$. Component 7 , representing a diet high in saturated fat and low in polyunsaturated fat, calcium and phosphorus, was negatively associated with $\mathrm{FEV}_{1}, \mathrm{FVC}$ and $\mathrm{FEV}_{1} / \mathrm{FVC}(p=0.001,0.008$ and 0.019 respectively). Using linear regression, we explored the relationship between lung function and food items high in nutrients represented by components 1 and 7. For component 1 food items, white bread was negatively associated with $\mathrm{FEV}_{1}$ and $\mathrm{FVC}$ and banana was positively associated with $\mathrm{FEV}_{1} / \mathrm{FVC}$. For component 7, reduced fat milk was positively associated with FEV 1 and FVC, skim and soya milks were positively associated with FVC and full cream milk was negatively associated with $\mathrm{FEV}_{1} / \mathrm{FVC}$. These results indicate that a diet high in wholegrains and low-fat dairy may be beneficial for lung function.

\subsection{Adherence to a Mediterranean Diet and LDL-c Levels Are Independent Predictors of Depression in Older Adults: The MEDIS Study}

E. Georgousopoulou, N. D'Cunha, N. Naumovski, S. Tyrovolas, S. Piscopo, G. Valacchi, N. Tsakountakis, A. Zeimbekis, V. Bountziouka, E. Gotsis, G. Metallinos, D. Tyrovola, A. Foscolou, J. Tur, A. Matalas, C. Lionis, E. Polychronopoulos, L. Sidossis and D. Panagiotakos

Faculty of Health, University of Canberra, Canberra 2617, Australia

Low cholesterol and poor diet are associated with an increased risk of depression. We aimed to evaluate the relationship between cholesterol, diet quality, and symptoms of depression in older adults enrolled in the Mediterranean Islands (MEDIS) study between 2004 and 2015. The sample consisted of 2749 individuals of which 1380 were female (F) (mean age: $73.6 \pm 7.14$ years (y)), and 1369 were male $(\mathrm{M})$ (mean age: $74.8 \pm 7.49$ y). Participants were categorised based on their Geriatric Depression Scale (GDS) score, a 30-item measure of symptoms of depression (0-9: normal; 10-19: mild; 20-30: severe). Using the MedDietScore (range $=0-55$ ), the mean level of adherence to a Mediterranean diet (MD) was $33 \pm 5$ for all participants $(p=0.876)$ and was inversely associated with the GDS score (Pearson's $r=-0.109, p<0.001$ ). A normal GDS score was observed in $67 \%$ of 
participants $(\mathrm{F}=61 \% ; \mathrm{M}=73 \%)$, while $5 \%$ were severe $(\mathrm{F}=7 \% ; \mathrm{M}=3 \%)(p<0.001)$. Low-density lipoprotein cholesterol (LDL-c) was significantly different $(p=0.008)$ in severe $(106 \pm 44.8 \mathrm{mg} / \mathrm{dL})$ compared to mild depression $(129 \pm 40.3 \mathrm{mg} / \mathrm{dL})$ and normal individuals $(123 \pm 41.6 \mathrm{mg} / \mathrm{dL})$. Multinomial logistic regression analysis revealed higher levels of LDL-c was independently related to an increased likelihood of mild symptoms of depression compared to severe symptoms (Odd's ratio $(\mathrm{OR})=1.01,95 \%$ confidence interval $(\mathrm{CI})=1.00-1.02, p=0.04)$ following adjustment for age, MedDietScore, body mass index and sex. In the same multi-adjusted model, a higher MedDietScore independently increased the odds of a normal GDS score $(\mathrm{OR}=1.07,95 \% \mathrm{CI}=1.01-1.13, p=0.021$ ) compared to a severe score, independent of LDL-c. In older adults, high diet quality, determined by the MedDietScore was associated with lower symptoms of depression, and low LDL-c levels increased the risk of severe depressive symptoms.

\subsection{Consumption of LC n3 PUFA and Contribution of Diet Sources and Supplements in Middle-Aged to Older Adults}

\section{E. K. Tregoweth ${ }^{1}$, A. M. Coates ${ }^{1}$, D. Greaves ${ }^{1,2}$ and A. E. Smith ${ }^{1,2}$}

1 Alliance for Research in Exercise, Nutrition and Activity (ARENA), Sansom Institute for Health Research, School of Health Sciences, University of South Australia, Adelaide, SA, Australia

2 Cognitive Ageing and Impairment Neurosciences (CAIN), School of Psychology and Social Work, University of South Australia, Adelaide, SA, Australia

The Australian suggested dietary target (SDT) for long chain omega-3 polyunsaturated fatty acids (LCn-3PUFA) to reduce the risk of chronic disease is $430 \mathrm{mg} /$ day for women and $610 \mathrm{mg} /$ day for men. We examined the dietary patterns of middle-aged to older adults ( $\mathrm{n}=90$, between $50-80$ years, 37 males) and evaluated LCn-3PUFA consumption contribution from dietary sources and supplements. Cardiovascular disease (CVD) risk was calculated (determined by the presence of diagnosed hypertension, diabetes and hyperlipidaemia) and additionally participants were classified into three age categories: 50-59 (55.23 \pm 2.88 years), $60-69$ (66.65 \pm 1.91 years) and 70-80 (74.59 \pm 2.95 years). The consumption of foods and supplements containing LCn-3PUFA was assessed using a validated, self-reporting, 41-item, food-frequency questionnaire and the total amount of LCn-3PUFA compared with sex-specific SDT guidelines. A total of $60.3 \%$ of females met the SDT guidelines compared with $40.5 \%$ of males $(p=0.065)$. From total consumption, LCn-3PUFA was predominately sourced from fish (males $48.2 \%$, females $47.9 \%$ ), meat/eggs (males 34.5\%, females $29.9 \%$ ) and supplements (males $11.76 \%$, females $21.71 \%$ ). The proportion of females $(0.396)$ supplementing LCn3PUFA was significantly $(p=0.038)$ higher than males $(0.297)$. The proportion of females across each age category consuming LC $n$-3PUFA supplements increased $(p=0.0076$, proportions: $50-59=0.125$, $60-69=0.400,70-80=0.647)$ and similar increases in supplement usage were evident in people with diagnosed CVD risk across all age categories ( $p=0.008$, proportions: 50-59 $=0,60-69=0.368,70-80=$ 0.625). Increased supplementation for both females and people with increased CVD risk is potentially attributed to effective public health messages and a heightened awareness of health with age.

\subsection{Predictors of Vegetable and Fruit Intake among Australian Children Aged 18 Months}

K. E. Lacy ${ }^{1}$, K. J. Campbell ${ }^{1}$, D. A. Crawford ${ }^{1}$, R. J. Wyse ${ }^{2,3,4,5}$, A. C. Spence ${ }^{1}$ and S. A. McNaughton ${ }^{1}$

1 Institute for Physical Activity and Nutrition (IPAN), School of Exercise and Nutrition Sciences, Deakin University, Geelong, Australia

2 Hunter New England Population Health, Wallsend, New South Wales, Australia

3 School of Medicine and Public Health, University of Newcastle, Callaghan, New South Wales, Australia

4 Hunter Medical Research Institute, Newcastle, New South Wales, Australia

5 Priority Research Centre for Health Behaviour, The University of Newcastle, New South Wales, Australia

Suboptimal consumption of vegetables and fruit by young children is common. Identifying predictors of vegetable and fruit intake is vital for informing strategies to promote adequate consumption from early in life. We examined predictors of vegetable and fruit intake among toddlers 
using secondary analyses of longitudinal data from the Melbourne Infant Feeding, Activity, and Nutrition Trial (InFANT) Program. Children's $(n=361)$ vegetable and fruit intake were assessed using two or three 24-h dietary recalls when children were aged 18 months. Potential predictors were selected based on the 2008 model of the home food environment pertaining to childhood obesity and more recent research. These were assessed at child ages four and nine months using a range of existing and purpose-designed measures addressing maternal education, vegetable/fruit consumption, nutrition knowledge and self-efficacy, home availability of vegetables/fruits, mother eating with her child, offering her child different foods and coping with life, breastfeeding and child's age of introduction to solids. Relationships between predictors and vegetable and fruit intakes were assessed using bivariate and multivariable linear regression. The home availability of vegetables at child age nine months was found to be predictive of vegetable intake at 18 months of age and maternal education and the home availability of fruits at child age nine months were predictive of fruit intake at 18 months of age. None of the other predictors examined were associated with intake. Strategies to support adequate vegetable and fruit intake among young children should encourage the availability of these foods in the home from early in life.

2.153. Evaluation of Macronutrient Intake during a Window of Opportunity Period among Stunted Infants: A Retrospective Cohort Study

D. M. Simadibrata ${ }^{1}$, P. A. Widyastuti ${ }^{1}$ and S. Bardosono ${ }^{2}$

1 Faculty of Medicine Universitas Indonesia, Indonesia

2 Department of Nutrition, Faculty of Medicine Universitas Indonesia-Cipto Mangunkusumo Hospital, Indonesia

The first two years of life serve as a critical window of opportunity for the appropriate growth and development of children. It is hypothesized that the main contributing factors to stunting are malnutrition in the window of opportunity. However, the profile of stunted infants in Jakarta and their relationship to dietary nutrient intake during the window of opportunity period has yet to be studied. Therefore, the aim of this study was to examine the dietary nutrient profile of stunted children in relation to the macronutrient intake. A retrospective cohort study was conducted among 179 infants aged two years and below from selected urban poor areas of Jakarta between February 2014 and March 2015 by obtaining anthropometric measurements and 24-h dietary recall data. Stunting was classified according to the World Health Organization as moderate stunting (heightfor-age Z-score $<-2 \mathrm{SD}$ ) and severe stunting (height-for-age Z-score $<-3 \mathrm{SD}$ ). An adequate macronutrient intake was determined as greater or equal to the data from the Recommended Dietary Allowance for Indonesian in 2013. Among the 179 infants, the number showing moderate stunting in the succeeding months increased from $12(6.7 \%)$ in the $8-10$ months group to $33(18.4 \%)$ in the two years of age group, while severe stunting increased slightly. The frequency of inadequate intake of energy, protein, carbohydrates and fat increased drastically from the first month of the monitoring period (aged 8-10 months) to the twelfth month. The frequency of inadequate intake of energy, protein, carbohydrates and fat followed a similar trend to the increase in moderate stunting.

\subsection{Developing and Embedding Nutrition Education in Elementary Schools in Yogyakarta, Indonesia}

L. Matwiejczyk ${ }^{1}$, Jumarko ${ }^{2}$, M. Widiharto ${ }^{3}$ and D. C. Sulistyoningrum ${ }^{4}$

1 Discipline of Nutrition and Dietetics, College of Nursing and Health Sciences, Flinders University, Adelaide, SA, Australia

2 Seyegan Community Health Center (Puskesmas Seyegan), Sleman

3 Tempel 1 Community Health Center (Puskesmas Tempel 1), Sleman

4 Department of Nutrition and Health, Faculty of Medicine Universitas Gadjah Mada, Yogyakarta, Indonesia

Yogyakarta, Indonesia, is experiencing the double burden of malnutrition, with under- and over-nutrition co-existing in young children at the national, district, community and household level. Malnutrition is associated with significant adverse health and economic consequences as well as an increased risk of morbidity and mortality in children and in later life as adults. Formative research in 
the sub-district of Seyegan, Sleman Regency, Yogyakarta found that young children lacked nutrition knowledge, 25\% missed breakfast, schools struggled with healthy eating guidelines and there was poor enforcement of Interior Ministry regulations relating to supplementary feeding. In addition, increasing food poisoning incidents in children and Indonesian Food and Drugs Administration data suggested that some snacks sold by school food vendors contained dangerous unregulated chemicals. This study describes the process of how a pilot program was developed to promote healthy eating in children through the school curriculum. In February 2017, program developers visited Australian schools and investigated school curriculum initiatives as Australian Award Fellows. In Yogyakarta, teachers and representatives from peak bodies were consulted and participated in workshops to develop an initiative to educate children. Teachers in collaboration with government experts committed to nutrition education in 30 pilot elementary schools. Commitment was also secured from the Ministries of Education, Health, Women Empowerment, Child Protection, and Planned Parenthood. As a result of a collaborative approach, elementary schools in Sleman Regency, Yogyakarta will be implementing a nutrition education program through the school curriculum with support across all of the ministries to promote healthy eating and enforce protective food-related policies.

\subsection{Maternal Supply of VLDL Polyunsaturated Fatty Acids to the Fetus Is Two-Fold Higher in} Preeclampsia than Control Pregnancy

\section{B. Meyer, S. Eather, S. Brown, T. Mitchell and D. Freeman}

University of Wollongong, Wollongong 2522, Australia

The fetus requires lipids provided by the maternal circulation for growth. We have previously shown that maternal and offspring polyunsaturated fatty acid (PUFA) status is reduced in preeclampsia (PE) and intrauterine growth restriction (IUGR), which may result from an abnormality in the maternal lipid supply. The aim was to compare maternal VLDL lipid composition in PE, IUGR and healthy controls. Maternal VLDL was isolated by ultracentrifugation from third trimester fasted plasma samples taken from women with PE $(n=16)$, IUGR $(n=7)$, and healthy controls $(n=47)$. VLDL triacylglycerol, total cholesterol and apolipoprotein B (apo B) were assessed using commercial kits. VLDL fatty acids were extracted and quantitated using gas chromatography. One way ANOVA was used to determine differences between the three groups, with Tukey post hoc tests to identify which groups were significantly different at $p<0.05$. The maternal VLDL particle number was 68$74 \%$ higher in PE (apo B $0.33 \mathrm{~g} / \mathrm{L}$ ) compared to control (apo B $0.19 \mathrm{~g} / \mathrm{L})(p=0.001$ ) and IUGR (apo B $0.20 \mathrm{~g} / \mathrm{L})(p=0.003)$ and maternal VLDL total PUFA was also increased in PE $(380 \mathrm{ug} / \mathrm{mL})$ over controls $(210 \mathrm{ug} / \mathrm{mL})(p=0.001)$ and IUGR $(180 \mathrm{ug} / \mathrm{mL})(p=0.023)$; but when expressed per particle number the lipid and fatty acid composition did not differ between the three groups. Despite low maternal PUFA status, the maternal supply of VLDL PUFA to the fetus was two-fold higher in PE compared to controls and IUGR. This increased VLDL PUFA supply may be an adaptive response to mitigate the comprised fetal PUFA status.

\subsection{Monitoring Advanced Glycation End-Products in Children's Nutritional Milk Formulae}

C. Prosser ${ }^{1}$, E. Carpenter ${ }^{1}$ and A. Hodgkinson ${ }^{2}$

1 Dairy Goat Co-Operative (NZ) Ltd., Hamilton, New Zealand

2 AgResearch, Hamilton, New Zealand

The production of milk formulae for infant nutrition involves high temperature processing. While necessary for microbiological safety, the exposure of milk to high temperatures generates advanced glycation end-products (AGEs). AGEs are being investigated for associations with food allergies and diabetes. $\mathrm{N}$-carboxymethyllysine (CML) is an example of an AGE formed between the amino acid lysine and lactose during the heating of milk, reducing the bioavailability of lysine. The presence of CML provides a means of monitoring the production of AGEs during the manufacture of milk formulae. A range of powdered infant nutritional formulae were tested for CML, using a 
commercially available CML ELISA kit. These included cow or goat milk formulae (with and without whey adjustment) and hydrolysate formulae of cow milk-derived whey proteins. The CML concentrations ranged 300-fold in all the formulae tested (3-970 ng/mg protein). Formulae made with goat milk consistently contained lower CML levels than cow milk formula. In general, CML was elevated in formula made with added (cow or goat) whey proteins. CML in formula made with hydrolysates of whey was also elevated compared to formula made with intact proteins. This study shows that there is a very large variation in CML concentrations across different formula types and that whey addition is a significant contributor to CML. While the biological significance of the longterm intake by infants of AGEs is being debated, it is prudent to explore options for reducing AGEs in the production of formula for infants.

\subsection{Differences in Infant Feeding Practices between Mothers with and without Type 1 Diabetes}

R. L. Thomson 1, M. A. Penno 1, S. Toome 2, R. Battersby 2, L. C. Harrison 3, P. G. Colman ${ }^{4}$, J. Wentworth 3, E. Davis 5, A. Haynes 5, M. Harris 6, M. E. Craig 7, W. D. Rawlinson 8, P. Vuillermin 9, R. O. Sinnott ${ }^{10}$ and J. J. Couper ${ }^{1,11}$, on behalf of the ENDIA Study Group

1 Robinson Research Institute and Adelaide Medical School, University of Adelaide, Adelaide, SA, Australia

2 Department of Nutrition \& Food Services, Women's and Children's Hospital, North Adelaide, SA, Australia

3 The Walter and Eliza Hall Institute of Medical Research, Parkville, VIC, Australia

4 Department of Diabetes and Endocrinology, Royal Melbourne Hospital, Parkville, VIC, Australia

5 Telethon Kids Institute and Department of Endocrinology \& Diabetes, Princess Margaret Hospital for Children, Perth, WA, Australia

6 Endocrinology Department, Lady Cilento Children's Hospital, Brisbane, QLD, Australia

7 Institute of Endocrinology and Diabetes, The Children's Hospital at Westmead, Westmead, NSW, Australia

8 Serology and Virology Division, NSW Health Pathology, Prince of Wales Hospital, Randwick, NSW, Australia, and University of NSW, Sydney, Australia

9 Child Health Research Unit, Barwon Health, Geelong, VIC, Australia

10 Melbourne eResearch Group, University of Melbourne, Parkville, VIC, Australia

11 Endocrinology and Diabetes Department, Women's and Children's Hospital, North Adelaide, SA, Australia

Infant nutrition is key to optimal growth and development. We aimed to compare the feeding practices of infants born to mothers with and without type 1 diabetes (T1D). A total of 326 mothers $(\mathrm{T} 1 \mathrm{D}=179)$ completed an infant feeding diary weekly from birth until 12 months as part of the Environmental Determinants of Islet Autoimmunity (ENDIA) study, a prospective pregnancy to childhood cohort that follows infants genetically at risk of T1D. In the first days postpartum, $98 \%$ of infants received breastmilk and $81 \%$ of infants of T1D mothers compared to $33 \%$ of non-T1D mothers received infant formula $(p<0.001)$. A total of $52 \%$ of infants were breastfed at 12 months and $70 \%$ at six months, with fewer mothers with T1D breastfeeding at six months $(61 \% \mathrm{vs.} 80 \%, p=0.002)$. A total of $68 \%$ of the infants of T1D mothers received formula in the first month, compared with $32 \%$ of nonT1D $(p<0.001)$, and the use of formula continued to be higher in infants of T1D versus non-T1D mothers during the first 12 months $(p<0.001)$. The introduction of first solids was at $20.7 \pm 3.8$ weeks (range 9-33 weeks), with no difference between groups ( $p=0.7$ ). A total of $85 \%$ of the infants had their first solids by six months. Rice products, fruits and vegetables were the most common first solids. In conclusion, infants of T1D mothers were more likely to receive formula within days of birth and throughout the first 12 months, with a reduced rate of breastfeeding. Barriers to breastfeeding for mothers also coping with T1D and any impact on the development of the infant microbiome and child health need to be further explored.

2.158. The Colic, Irritability, Reflux, Intolerance and Allergy Study (CIRIAS): A Randomised Clinical Trial of Maternal Dietary Interventions for Colic in Breastfed Infants

Tracy Harb ${ }^{1}$, Pamela Dodrill ${ }^{2}$, Frances Connor ${ }^{3}$, Dr Sue Shepherd ${ }^{4}$ and Rebecca J. Hill ${ }^{1}$

1 The University of Queensland, Child Health Research Centre, Children's Nutrition Research Centre, Brisbane, Australia

2 Brigham and Women's Hospital, Boston, USA 
3 Lady Cilento Children's Hospital, Brisbane, Australia

4 Department of Rehabilitation, Nutrition and Sport: Dietetics and Human Nutrition, La Trobe University, Bundoora, Victoria, Australia

The CIRIAS project was a randomised clinical trial investigating the association between maternal diet and colic in fully breastfed infants aged four months or younger. Thirty mothers were randomised to two diet groups: a cow's milk and egg (CMP/Egg) elimination $(n=15)$, or the low FODMAP (LF) diet $(n=15)$. The mothers participated for four weeks: baseline data collection (week one); dietary intervention (weeks two and three); open food challenge (week four). The primary outcome data were parent-reported infant cry/fuss time (Barr Baby Diary); and bowel motion frequency (Amsterdam Stool Chart). Week four cry/fuss data were excluded from analysis due to poor compliance with recording. Overall, $47 \%$ of infants experienced a reduction in cry/fuss time of at least $25 \%$. A linear mixed model analysis was performed to assess the effect of diet type on cry/fuss time and bowel motion frequency across the three weeks. A significant reduction in cry/fuss time, as well as bowel motion frequency, was found over time ( $p=0.002$ and $p=0.039$, respectively), showing that both dietary interventions were effective for reducing cry/fuss times and bowel motion frequency in infants with colic. Although neither diet was found to be significantly more effective than the other, a larger reduction in cry/fuss time from Week 1 to Week 3 was observed for mothers in the CMP/egg group (mean reduction $-82.7 \mathrm{mins} / \mathrm{d}( \pm 126.3)$ ) than with the LF $\operatorname{diet}(-49.3 \mathrm{~min} / \mathrm{d}$ $( \pm 108.6)$ ). The diets may work to modulate infant gastrointestinal and immune function through reduced allergen load or an altered carbohydrate profile of thes maternal breastmilk.

\subsection{Do Weight Management Interventions Effect Self-Esteem in Overweight and Obese Youth: A Systematic Review and Meta-Analysis}

M. Murray, A. L. Dordevic and M. P. Bonham

Department of Nutrition, Dietetics and Food, Monash University, Victoria 3168, Australia

Building self-esteem in overweight adolescents is key to long-term weight management, yet selfesteem is rarely a key outcome of adolescent weight management interventions. This systematic review and meta-analysis investigates the impact of multicomponent weight management interventions on self-esteem in overweight and obese adolescents. Six databases were searched in December 2014. Eligible studies met the following criteria: (1) randomized controlled trial, (2) overweight or obese participants, (3) adolescents (10-19 years), (4) multicomponent weight management intervention, and (5) reported self-esteem and weight changes. Thirteen studies with 1157 overweight or obese adolescents, aged 10-19 years, were included. Meta-analyses showed no significant change in self-esteem $(0.27$ [ $-0.04,0.59])$, but a reduced body mass index z-score following intervention $(-0.17[-0.22,-0.11])$. The lack of change in self-esteem suggests weight loss alone is insufficient to improve self-esteem. Multicomponent weight management interventions require a specific focus on self-esteem to improve this outcome in overweight and obese adolescents.

\subsection{Relationship between Erythrocyte Membrane Phospholipid Fatty Acids and Sleep Disturbance in Chinese Children and Adolescents}

J. Tang ${ }^{1}$, Y. K. Yan ${ }^{2}$, J. S. Zheng ${ }^{3}$, J. Mi ${ }^{2}$ and D. $\mathrm{Li}^{1,4}$

1 Department of Food Science and Nutrition, Zhejiang University, Hangzhou, China

2 Department of Epidemiology, Capital Institute of Pediatrics, Beijing, China

3 Medical Research Council Epidemiology Unit, University of Cambridge, Cambridge, UK

4 Institute of Nutrition \& Health, Qingdao University, Qingdao, China

The association of circulating fatty acid (FA) compositions with sleep quality have so far been unclear. We aimed to investigate the associations between erythrocyte membrane FA compositions and the prevalence of sleep disturbance in children and adolescents. A cross-sectional study was conducted among 2337 Chinese school-aged children and adolescents. The presence of sleep disturbance was self-reported and parent-reported by standardized questionnaires. Erythrocyte FAs 
were measured by gas chromatography, and desaturase activities were estimated by FA ratios. Multivariable-adjusted odds ratios (ORs) and 95\% confidence intervals (CIs) for sleep disturbance across FA quartiles were calculated by logistical regression models. We found significantly higher proportions of 24:0, 24:1n-9, 20:3n-3, while there were significantly lower proportions of 18:1n-9, 22:5n-3, 22:6n-3 and D9D-18 in cases compared with controls. After controlling for sociodemographic, anthropometric, clinical parameters and dietary factors, total $\mathrm{n}-3$ polyunsaturated FA (PUFA), 22:5n-3 and 22:6n-3 were inversely associated with sleep disturbance, with highest versus lowest quartiles ORs and 95\% CIs as $0.57(0.40,0.82), 0.69(0.48,0.99)$ and $0.69(0.49,0.96)$, respectively. The per one SD difference of each FA, OR and $95 \%$ CI were $0.91(0.86,0.97)$ for total n-3 PUFA, 0.91 $(0.83,0.99)$ for $22: 5 n-3$, and $0.92(0.86,0.99)$ for $22: 6 n-3$, respectively. The present study suggests that n-3 PUFA, especially 22:5n-3 and 22:6n-3, are inversely associated with the prevalence of childhood sleep disturbance.

\subsection{Healthy Eating and Lifestyle Program (HELP): Gender Differences in a Two-Year, State-Wide Evaluation}

K. C. Langston ${ }^{1}$, R. Hay ${ }^{2}$, A. Byrnes ${ }^{2,3}$ and L. R. Ross ${ }^{1,2,4}$

1 Griffith University, Australia

2 Royal Brisbane and Women's Hospital, Australia

3 University of Queensland, Australia

4 Menzies Health Institute Queensland, Australia

Effective long-term lifestyle intervention programs are needed to manage obesity and chronic disease risks. The Healthy Eating and Lifestyle Program (HELP) was a multidisciplinary lifestyle behaviour change intervention conducted at Queensland hospital outpatient centres for weight management in high-risk patients. This study aimed to evaluate short- and long-term weight, quality of life (QoL) and self-efficacy outcomes for men and women attending HELP. Patients with a body mass index $(\mathrm{BMI}) \geq 25 \mathrm{~kg} / \mathrm{m}^{2}$ attending various hospital-based clinics in 2014-2015 were invited to participate in HELP. Data on weight, QoL and self-efficacy were collected at the first (initial) and last HELP session (review) and in a two-year follow-up study. Among participants with complete weight data at initial, review and two years $(n=115$ of $493,23 \%)$, mean weight loss was clinically and statistically significant at review $(-2 \mathrm{~kg})$ and two years $(-4 \mathrm{~kg})(p \mathrm{~s}<0.01)$. Clinically significant $5 \%$ weight loss was achieved by $40 \%$ of the included patients at two years. Significant weight loss was demonstrated across genders and BMI classes, with no differences between subgroups ( $p \mathrm{~s}>0.05$ ). However, significant improvements in QoL and self-efficacy were confined to those with more severe obesity ( $p s<0.05)$. Males improved in physical QoL $(p<0.01)$, while females improved in mental QoL $(p<0.01)$. Females had poorer initial self-efficacy $(p<0.01)$, with significant improvement at two years $(p<0.01)$. While the results support secondary prevention programs for the reduction of chronic disease risk and progression, the likely benefits to patients and the Australian health system require further research to explore clinical outcomes for all patients entering the program.

\subsection{The Nutritional Content of Supermarket Beverages: An Analysis of New Zealand, Australia, Canada and the UK \\ Lynne Chepulis ${ }^{1}$, Shaunie Hill ${ }^{1}$, Jason $\mathrm{Wu}^{2}$, Jodi Bernstein ${ }^{3}$ and Gael Mearns ${ }^{4}$ \\ 1 ToiOhomai Institute of Technology, New Zealand \\ 2 George Institute for Global Health, Australia \\ 3 University of Toronto, Canada \\ 4 Auckland University of Technology, New Zealand}

Sugar-sweetened beverages are a major contributor to obesity, yet it is unknown how these products vary in different western countries. Nutritional information was collected from beverages in major retailers in New Zealand, Australia, Canada and the UK. Data was analysed for nutritional content, serving size, proportion of sugar-free products as well as the number of products that 
exceeded WHO levels of free sugars, and/or would potentially be taxable based on the proposed UK sugar tax levy.

A total of 4157 products were analysed. New Zealand had the highest number of beverages with sugar added to them $(52.3 \% ; p \leq 0.0001)$, whilst Canada had the most beverages that were sugar-free $(21.9 \%, p<0.01)$. Differences in energy, carbohydrate and sugar content were observed both between countries and within categories, with the UK generally having a significantly lower energy and sugar content. Up to half of all products across categories/countries exceeded the serving size recommendations, and more than two thirds of the single serve products across the four countries provided more than $10 \%$ of an adult's daily energy requirement as sugar. At least half of all beverages in the four countries were eligible for sugar taxation, with the UK having the lowest proportion of products in both the low tax (5-8\% sugar) and high tax ( $>8 \%$ sugar) categories.

In conclusion, readily-available, high sugar beverages continue to dominate the market in these countries, though the UK appears to be making efforts towards reducing the sugar content of common beverage categories.

\subsection{Comparison of Findings from a Community-Based Partial Meal Replacement Program with and} without a Supportive Mobile Phone Application

G. James-Martin, G. Hendrie, D. Silvera Tawil, E. Brindal, J. Freyne and M. Noakes

CSIRO Health \& Biosecurity, Australia

Partial meal replacement programs can achieve significant weight loss in a clinical setting; however their effectiveness in a community pharmacy setting is unknown. Impromy ${ }^{\mathrm{TM}}$ is a partial meal replacement program that combines face-to-face pharmacy support with an interactive mobile phone application (app). The app provides users with the ability to record food intake, prompts selfmonitoring of weight, and uses a rewards system to reinforce positive behavior change. Since the launch of the program in 2014, data has been collected through a national pharmacy database (Guildcare) and through the Impromy ${ }^{\mathrm{TM}}$ app for all individuals who have joined the program. Percentage weight loss was calculated as change between the first and last recorded pharmacy visit (measured by pharmacy staff) and first and last weight entered into the app (self-reported). Over the 21 month period since launch, April 2014 to December 2016, 27,608 individuals joined the program. Of those, 14,073 (51\%) have visited the pharmacy and used the app, 11,736 (43\%) visited the pharmacy only, and $1799(7 \%)$ used the app only. The average duration on the program for all users was 35.6 days (5.1 weeks). The average recorded weight loss for all participants was $1.6 \%$ of body weight per month (1.5\% for pharmacy only, $1.7 \%$ for pharmacy + app). Weight loss increased with duration on the program. Average self-reported weight loss was higher (2.5\% of body weight per month) for users of the app. The additional support provided by an interactive app appears to increase weight loss in a pharmacy-delivered partial meal replacement program.

\subsection{Hypertension: Its Prevalence and Association with Obesity among Indigenous Adolescents of Sarawak, Malaysia}

\section{W. L. Cheah, C. T. Chang, Helmy, Hazmi, Razitasham, Safii, Grace Kho and Woei Peng}

Faculty of Medicine and Health Sciences, Universiti Malaysia Sarawak, Malaysia

The association between obesity and hypertension has been largely reported in the adult population, but limited for adolescents, particularly in Sarawak. The objective of this study is to determine the prevalence of hypertension and its association with obesity among indigenous adolescents aged 12-17 years in Sarawak. It was a cross-sectional study using a questionnaire, anthropometric and blood pressure measurement. Using a systematic sampling procedure, a total of 18 secondary schools representing urban and rural areas from 11 divisions of Sarawak were selected. Data was analyzed using Statistical Package for Social Sciences Program (SPSS) version 22.0. A total of 1780 secondary school children participated in the study with $58.9 \%$ females and $20.1 \%$ reported at least one parent with a history of hypertension. The prevalence of pre-hypertension was $13.4 \%$, 
stage 1 hypertension was $13.5 \%$ and stage 2 hypertension was $4.7 \%$. The prevalence of overweight and obesity was $24.4 \%$, elevated waist circumference was 13.4 , and overfat and obese was $6.2 \%$. The prevalence of hypertension among male respondents was $23.9 \%$, for female respondents this was $14.1 \%$. In multiple logistic regression, adjusting for age and family history, hypertension was associated with sex (males) $(\mathrm{OR}=3.20, p<0.000)$, ethnic group ( $\mathrm{ref}=\mathrm{Iban}$, Malay, $\mathrm{OR}=0.72, p=0.015$; Bidayuh, $\mathrm{OR}=0.65, p=0.019)$, overweight and obese $(\mathrm{OR}=3.311, p<0.000)$, elevated waist circumference $(\mathrm{OR}=2.15, p<0.000)$, overfat and obese $(\mathrm{OR}=2.08, p=0.004)$. Hypertension and obesity in this population is highly prevalent. Screening blood pressure and the assessment of nutritional status can be recommended as the routine health assessment in school health programmes to detect and provide early intervention for those at risk of hypertension

\title{
2.165. A Systematic Review of the Effects of Alcohol Consumption on Food Energy Intake in Adults
}

A. Kwok, G. Paton, A. Dordevic and H. Truby

Department of Nutrition, Dietetics and Food, Monash University, Notting Hill, VIC 3168, Australia

Overweight is increasing worldwide, predisposing a greater number of people to a myriad of lifestyle diseases. Due to its high energy density, alcohol consumption is a contributor to weight gain. There is growing evidence that alcohol's effects on appetite and subsequent food intake may contribute to positive energy balance. This systematic review aimed to explore the effects of alcohol consumption on food energy intake in adults. Four databases (Ovid Medline, CINAHL, Embase and PsycINFO) were searched systematically. The search was limited to crossover trials and randomised controlled trials for healthy adults where an alcohol dose was compared against a non-alcohol condition. Study quality was assessed using the Effective Public Health Practice Project tool. Of the 16,045 studies considered, 22 were included. The results suggest that an alcohol dose has varying effects on subsequent food energy intake. Studies consistently demonstrated that voluntary dietary energy intake did not decrease as a result of alcohol energy ingestion. Seven out of ten studies showed that alcohol consumption increased food and preload energy intake when compared to a no-energy pre-load or no pre-load, with those giving a loading dose of alcohol $\geq 30 \mathrm{~g}$ or $0.6 \mathrm{~g} / \mathrm{kg}$ more likely to demonstrate an increase in food and pre-load energy intake. Although the effects of alcohol consumption on energy intake are inconsistent, this review highlighted that adults do not compensate for alcohol energy by eating less, which may have implications for the energy balance. Studies with larger samples sizes and consistent methodological reporting are warranted to provide conclusive evidence.

\subsection{The CSIRO Healthy Diet Score Survey Shows Differences in Compliance with Dietary Guidelines by} Weight Status

\author{
G. A. Hendrie ${ }^{1}$, R. K. Golley ${ }^{2}$ and M. Noakes ${ }^{1}$ \\ 1 CSIRO Health and Biosecurity, Adelaide, SA, Australia \\ 2 School of Pharmacy and Medical Sciences, University of South, Adelaide, SA 5000, Australia
}

There are few dietary assessment tools that are scientifically developed and freely available online. The CSIRO Healthy Diet Score survey asks about the quantity of five food groups (fruit, vegetables, grains, meat and dairy) and discretionary choices (such as cakes, biscuits, and takeaways), as well as the variety of foods consumed. Self-reported intake was calculated and compared to the age/gender recommended food group servings. On completion, individuals receive a personalised diet score, reflecting their overall compliance with the Australian Dietary Guidelines. The survey was launched in May 2015, and as a result of four media releases, over 183,000 Australians have completed the survey. The majority of respondents were female $(71 \%)$, with an even distribution across age groups. Almost half (48.2\%) of the respondents were classified as normal weight, $30.4 \%$ overweight and $18.9 \%$ obese. The average diet score was 58.6 out of a possible $100(\mathrm{SD}=12.9)$. The average diet score for normal weight, overweight and obese adults was 60.5, 58.1 and 56.7, respectively. Obese adults scored $26 \%$ lower than normal weight adults for discretionary foods, $18 \%$ 
lower for fruit and 13\% lower for healthy fats. Scores for meat and grains were similar by weight status. Regression analysis showed that the diet score was negatively associated with body mass index $(\beta=-0.183, p<0.001)$, after controlling for gender and age. The guidelines are designed to promote wellbeing within the population. The results of this large survey suggest that obese Australians have poorer diet quality. This survey provides a cost effective way to monitor population diet quality and compliance with guidelines.

\subsection{Relationship between Spicy Flavor, Spicy Food Frequency and Obesity in the Chinese Rural} Population: The Ruraldiab Study

K. Yang ${ }^{1}$, Y. $\mathrm{Li}^{1}$, X. Liu ${ }^{1}$, S. Yu ${ }^{2}$, Z. Mao ${ }^{1}$, R. Tu ${ }^{1}$, W. $\mathrm{Li}^{2}$ and C. Wang ${ }^{1, *}$

1 Department of Epidemiology and Biostatistics, College of Public Health, Zhengzhou University, Zhengzhou, China

2 Department of Nutrition and Food Hygiene, College of Public Health, Zhengzhou University, Zhengzhou, China

* Corresponding Author

Previous studies have explored the relation between spicy food consumption and obesity, but the evidence remains limited and inconsistent, especially for rural populations. The purpose of this study was to explore the association between spicy flavor, spicy food frequency and general obesity in rural Chinese adults. A total of 15,698 subjects aged 35-74 years from the Rural Diabetes, Obesity and Lifestyle (RuralDiab) Study (Chinese Clinical Trial Registry: ChiCTR-OOC-15006699) were recruited for this study. Analysis of co-variance was used to determine the group differences in BMI parameters. Logistic regression was used to analyze the association between spicy flavor, spicy food frequency and general obesity. The crude and standardized prevalence of obesity were $16.79 \%$ and $17.59 \%$, respectively. There were increasing trends in the prevalence of obesity with the level of spicy flavor and the frequency of spicy food (all $P_{\text {trend }}<0.001$ ). Compared with no spicy flavor, the adjusted ORs $(95 \%$ CIs) of mild, middle and heavy for general obesity were 1.231(1.116-1.357), 1.470(1.296$1.666)$ and 1.608(1.308-1.978), respectively $(P$ for trend $<0.001)$. Similarly, as compared with never eating spicy food, the adjusted ORs (95\% CIs) for 1 or 2 days/week, 3 to 5 days/week and 6 or 7 days/week were 1.092(0.731-1.630), $1.280(0.923-1.777)$ and $1.252(1.027-1.527)$, respectively $\left(P_{\text {trend }}=\right.$ 0.023). The present study indicated that the spicy food consumption was positively associated with the risk of obesity in Chinese rural adults, especially regarding the characters of spicy flavor. Further studies are needed to explore the mechanism of the association.

\subsection{Evaluation of a Pictorial Dietary Assessment Tool for Hospitalized Patients with Diabetes: Cost, Accuracy and User Satisfaction}

\section{Budiningsari, S. Shahar, Z. Abdul Manaf, N. Mohd Nordin and S. Susetyowati}

Department of Nutrition and Health, Faculty of Medicine, Universitas Gadjah Mada, Yogyakarta 3471070, Indonesia

Although nutritional screening and dietary monitoring in clinical settings are important, studies on related user satisfaction and cost are still lacking. This study aimed to elucidate the cost of implementing a newly developed dietary monitoring tool, the Pictorial Dietary Assessment Tool (PDAT), and investigated the accuracy of estimation and user satisfaction of the PDAT. A cross-over intervention study was conducted among 132 hospitalized patients with diabetes. Cost and time for the implementation of PDAT in comparison with Comstock was estimated using the activity-based costing approach. Accuracy was expressed as the percentage of energy and protein obtained by both methods, which were within $15 \%$ and $30 \%$ of those obtained by the food weighing. The satisfaction of healthcare staff was measured using a standardized questionnaire. The time to complete the food intake recording of patients using PDAT $(2.31 \pm 0.70 \mathrm{~min})$ was shorter than Comstock $(3.53 \pm 1.27$ $\min )(p<0.001)$. The overall cost per patient was slightly higher for PDAT (USD $0.27 \pm 0.02)$ than for Comstock (USD $0.26 \pm 0.04)(p<0.05)$. The accuracy of the estimation of energy and protein of using 
Comstock was lower than that of PDAT (energy: $>86 \%$ vs. $>96 \%$, respectively; $p<0.05$; protein: $<40 \%$ vs. $>71 \%$, respectively; $p<0.05$ ). The mean score for user satisfaction was higher for PDAT than for Comstock $(p<0.05)$. PDAT required a shorter time to be completed and rated better than Comstock. Only USD 0.01 is needed to increase the accuracy of the estimation of energy intake by $10 \%$ and protein intake by $30 \%$ when using PDAT.

\subsection{Nutrient Intake and Adherence to Dietary Guidelines in Haemodialysis Patients in New Zealand: Room for Improvement}

\section{Z. Xie ${ }^{1}$, R. M. McLean ${ }^{2}$ and M. R. Marshall ${ }^{3}$}

1 Dept Human Nutrition, University of Otago, Dunedin 9054, New Zealand

2 Dept Preventive and Social Medicine, University of Otago, Dunedin 9054, New Zealand

3 School of Medicine, University of Auckland, Department of Renal Medicine, Counties Manukau District Health Board, Auckland 1010, New Zealand.

Patients undergoing haemodialysis have a high rate of mortality, mostly due to cardiovascular disease. Current renal-specific guidelines contain a number of nutrient recommendations, including the restriction of dietary sodium. This observational study describes baseline nutrient intake in a multi-ethnic sample of 85 adult haemodialysis patients participating in the SoLID Trial in New Zealand. Three-day weighed food records were analysed using Foodworks 8 Professional food composition database, supplemented by other sources of nutrient information. Sixty-six percent of the participants were male, with a mean (SD) age of 52 (13) years. The largest ethnic group was 'New Zealand European and Others' (45\%) followed by Pacific (29\%). One third of participants had a diagnosis of diabetes mellitus. Mean (SD) sodium intake was 2502 (957) mg/day (2591 mg/day for men and $2330 \mathrm{mg} /$ day for women). Only $24 \%$ of participants met the recommended level of sodium intake, with $54 \%$ exceeding the recommended sodium intake range. Sodium intake was positively associated with energy intake $(p<0.001)$. Protein intake contributed around $18 \%$ of total energy. Saturated fat intake contributed a mean of $13 \%$ total energy intake, and mean carbohydrate intake accounted for $47 \%$ of total energy. About two thirds of participants were consuming inadequate fibre and around half of participants reported excess phosphorus intake. A high proportion of dialysis patients in the SoLID Trial did not meet the current renal-specific dietary recommendations for a range of nutrients. A total diet approach is needed to lower sodium intake and improve total diet quality among haemodialysis patients in New Zealand.

\subsection{Comparison of Predicted Energy Requirements with Total Energy Expenditure Measured by the Doubly-Labelled Water Method in Acute Spinal Cord Injury}

K. Desneves, J. Rafferty, H. Rodi, M. Panisset, L. Ward, A. Nunn, D. Brown and M. Galea

1 Department of Nutrition and Dietetics, Austin Health, Heidelberg Victoria 3084, Australia

2 Department of Medicine, Royal, Melbourne Hospital, The University of Melbourne, Parkville VIC 3000, Australia

3 School of Chemistry and Molecular, Biosciences, The University of Queensland, St Lucia QLD 4072, Australia

4 Victorian Spinal Cord Service, Austin Health, Heidelberg Victoria 3084, Australia

5 Spinal Research Institute, Austin Health, Heidelberg Victoria 3084, Australia

The estimation of energy requirements following acute spinal cord injury (SCI) is critical for the provision of appropriate nutritional interventions, however simple but valid prediction equations are lacking in this population.

The aim of this study was to evaluate the agreement between reference measurements of total energy expenditure (TEE) using doubly-labelled water (DLW) and six prediction equations commonly used to estimate energy requirements in acute SCI, namely the Harris Benedict, Schofield, Henry, Nelson and Buchholz equations and the ratio method $(\mathrm{kJ} / \mathrm{kg})$. 
Twenty participants (18M:2F) completed DLW and anthropometric assessments. Body fat-free mass and fat mass were determined by deuterium dilution. TEE was calculated from the estimated energy requirements (EER) using a stress factor of 1.2 and an activity factor of 1.1.

The median TEE was 9.1 MJ (IQR 8.2-11.4 MJ). Agreement between EER and TEE was generally poor with concordance correlation coefficients ranging from 0.15 for the Schofield equation to 0.31 for the Bucholz equation and 0.36 for the Nelson prediction equation. The limits of agreement (LOA) were wide for most predictions, the best performing equation being that of Bucholz, with $0.8 \mathrm{MJ}$ bias when compared to DLW, but the LOA remained large $( \pm 35 \%)$.

The equations in common use to clinically estimate energy expenditure in acute SCI patients generally exhibit poor accuracy compared with the DLW measurements of TEE. FFM-based equations performed marginally better than the simpler anthropometric-based equations, but the wide LOA precludes their use in individual subjects, a necessary prerequisite for clinical practice. Indirect calorimetry remains the preferred method for determining energy requirements.

\title{
2.171. Nutritional Status of Vascular Surgery Patients: Can Popular Malnutrition Screening Tools Identify
} Those at Risk?

\author{
J. M. Thomas ${ }^{1}$, J. Suen ${ }^{1}$, C. Delaney ${ }^{2}$, I. Spark ${ }^{2,3}$ and M. Miller ${ }^{1}$ \\ 1 Nutrition and Dietetics, Flinders University, Bedford Park, SA, Australia \\ 2 Department of Vascular Surgery, Southern Adelaide Local Health Network, SA, Australia \\ 3 Department of Vascular Surgery, Flinders University, Bedford Park, SA, Australia
}

Vascular disease is a growing problem and while the links between obesity/overweight and vascular disease are well established, undernutrition can be an issue with a significant impact on clinical outcomes and thus should be identified expediently. We aimed to investigate the nutritional status of patients with vascular disease and to determine the concurrent and predictive validity of the Malnutrition Universal Screening Tool (MUST), Malnutrition Screening Tool (MST), MiniNutritional Assessment-Short Form (MNA-SF) and Nutrition Risk Screen-2002 (NRS-2002). Nutritional status was assessed using the Patient-Generated Subjective Global Assessment (PGSGA) and a dietitian's assessment incorporating micronutrient status. The risk of malnutrition varied from 12.5 (MUST) to $47.5 \%$ (MNA-SF) in the 323 participants ( $69 \%$ male, mean age $67.6 \pm 14.1$ y) and the PGSGA rated $15.5-20 \%$ as malnourished. Micronutrient deficiencies were prevalent with $79 \%$ showing low vitamin C levels, $56 \%$ with low vitamin D, and over $40 \%$ with low zinc, vitamin B12 and folate levels. The dietitian's assessment classified $75.5 \%$ as warranting further intervention. Concurrent validity varied, however the MNA-SF performed consistently better than the other tools, achieving a-priori levels against the PGSGA. All tools including the PGSGA performed poorly against the dietitian's assessment. Predictive validity was variable, however the NRS-2002 and the MNA-SF detected significant differences in the discharge destination, quality of life and hospital length of stay with at risk participants having poorer outcomes. The variable and inconsistent results indicate the need for alternative screening and assessment instruments, incorporating micronutrients, for use in these patients.

2.172. The Leguminous Apios Americana Medikus Improved the Nutrition State and Accelerated the LiverRegeneration of Orthotopic Hepatocellular Carcinoma in a Mouse Model That Underwent Hepatectomy

Yunyun Yang, Fujie Yan, Qiang Chu, Zihuan Zheng and Xiaodong Zheng *

College of Biosystems Engineering and Food Science Zhejiang University, Hangzhou 310058, China

* Corresponding Author

Surgical hepatectomy is the most effective treatment for hepatocellular carcinoma (HCC) in patients with well-preserved liver function, but postoperative malnutrition leads to a poor prognosis. The Apios Americana Medikus (AAM) was studied for its high nutritional potential and anticarcinogenic effects. This study used a mouse xenograft model of HCC with partial hepatectomy after induction. The AAM group had higher relative liver weights, faster liver regeneration rates and 
longer survival times than either the positive group or the control group. The serum levels of ALB, GLO and TP were increased significantly in the $40 \%$ group compared to the control group, whereas the levels of GSP, AKP and $\mathrm{Ca}^{2+}$ were comparable, albeit with a small increase $(p<0.05)$ in the AAM intervention group. The levels of CRP, IL-6, and TNF-alpha were slightly decreased and the levels of TRF and IL-10 were slightly increased $(p<0.05)$ in the AAM intervention group. Minor liver necrosis and deformity were evident in the $10 \%$ group; karyokinesis and liver regeneration were observed in the $20 \%$ and $40 \%$ groups. The number of PCNA positive cells was considerably lower in the $40 \%$ group (35.97 8.23 cells/field) than the control group (57.29 5.92 cells/field). In addition, the expression of TNF-alpha and IL-6 were inhibited, indicating that the circulating levels of these inflammatory cytokines were significantly reduced after the AAM intervention $(p<0.05)$. AAM has demonstrated anti-tumor and nutrition effects in xenografted HCC mice. Therefore, AAM may have a potential adjuvant nutrition effect in HCC recurrence prevention.

\title{
2.173. Compliance to Healthy Food Guidelines at Two New South Wales Public Hospitals
}

\author{
C. Y. Tsai ${ }^{1}$, E. Svensen ${ }^{2}$, V. Flood ${ }^{3,4}$, T. Nguyen ${ }^{1}$, P. H. Lai ${ }^{1}$, E. Sanad ${ }^{1}$, K. Suen ${ }^{1}$ and J. H. Y. Wu ${ }^{5}$ \\ 1 Faculty of Dentistry, University of Sydney, Sydney, NSW 2006, Australia \\ 2 Faculty of Science, Medicine \& Health, University of Wollongong, Wollongong, NSW 2522, Australia \\ 3 Western Sydney Local Health District, North Parramatta, NSW 2151, Australia \\ 4 Faculty of Health Sciences, University of Sydney, Sydney, NSW 2006, Australia \\ 5 The George Institute for Global Health, University of New South Wales, Sydney, NSW 2052, Australia
}

(1) Background: In seeking to improve the food environment in public health facilities, the Ministry of Health, New South Wales (NSW), Australia, has developed mandatory nutrition standards for foods/beverages in NSW health facilities. Revisions were made in 2017 to reflect changes in the national food policy context, and we aimed to assess the level of compliance of two major Western Sydney public hospitals with the updated standards. (2) Methods: The first of two cross-sectional assessments was conducted (February 2017) across 11 food outlets in the hospitals using an audit tool to assess compliance with the current policy directive related to the availability, placement, and promotion of 'occasional' products (corresponding to discretionary items defined by the Australian Dietary Guideline). (3) Results: Availability: Adherence was low for overall food availability with only $1 / 11(9 \%)$ sites compliant, defined as $<25 \%$ 'occasional' products. 'Occasional' foods/drinks made up $57 \pm 21 \%$ (mean \pm SD) and $49 \pm 16 \%$ of products available. Placement: On average, food outlets did not meet recommendations of limiting 'occasional' foods in prominent positions: 'Occasional' items made up nearly $50 \%$ of 'point of sale' and 33\% 'counter top' products. Promotion: Meal deals for staff at both hospitals included 'occasional' products, which is not compliant. (4) Conclusion: The baseline audit indicates that substantial improvements in availability, placement, and promotion will be needed at these public hospitals to meet the mandatory standards. Audits of other NSW health facilities using the developed tool are needed to investigate similarities and differences in compliance between facilities. Ongoing tracking will help to inform whether the revised standards are leading to improved food environments in health facilities.

\subsection{Characteristics of Dietary Intake and Serum Albumin in Head and Neck Cancer Patients Undergoing Radiotherapy and Chemotherapy}

Hitomi Nagasaki ${ }^{1}$ and Michiko Nakamura ${ }^{2}$

1 Graduate School Department of Interdisciplinary Research, School of Nursing, University of Yamanashi, Yamanashi 400-8510, Japan

2 Kyoritsu Women's University Faculty of Nursing, Tokyo 101-8437, Japan

Patients with head and neck cancer often have a decreased dietary intake and serum albumin concentrations while undergoing radiotherapy or chemotherapy. In this study, we measured daily dietary intake and serum albumin concentrations in patients with head and neck cancer (hypopharyngeal cancer and others) currently undergoing radiotherapy or chemotherapy. The study 
participants included 13 inpatients undergoing radiotherapy (RT group) and 10 patients undergoing chemotherapy (CT group). Data were collected at three time points: before therapy, during therapy (after 40-Gy radiotherapy or one-week chemotherapy), and following therapy (after 70-Gy radiotherapy or two-week chemotherapy). Data collected included one-day intake of nutrients (total energy, protein, carbohydrates, fats) and food groups (grains, fish, and meat) as well as data on blood biochemical markers (serum albumin, TLC and RBC). The mean values of dietary intake and blood biomarkers were compared between time points within each study group. Excel Eiyo-kun software 6.0 (Kenpakusha, Tokyo, Japan) and SPSS Ver.20 was used. Protein intake was significantly lower after than during therapy in both groups (RT group, mean decrease of $45.4 \pm 16.6 \mathrm{~g}$; CT group, mean decrease of $44.1 \pm 15.8 \mathrm{~g}$ ). The intake of meat and fish decreased significantly in the RT group following therapy, whereas the intake of fish decreased significantly in the CT group during therapy. The serum albumin concentrations were significantly lower after than before therapy in both groups (RT $3.63 \pm 0.43 \mathrm{~g} / \mathrm{dl}$, CT $3.29 \pm 0.43 \mathrm{~g} / \mathrm{dl}$ ). The serum albumin concentrations in patients with head and neck cancer can be increased through an increased intake of protein-rich food during therapy.

\subsection{Exploring Practice Gaps to Improve PERIoperativE Nutrition Care of the Elderly (EXPERIENCE Study)}

A. Byrnes, A. Young, A. Mudge, M. Banks and J. Bauer

School of Agriculture and Food Sciences, Faculty of Science, University of Queensland, St Lucia QLD 4072, Australia

Older surgical patients are vulnerable to malnutrition and a decline in physical function. Delivering care that aligns with evidence-based best practice can confer benefits for the patient and the facility, however research translation into clinical practice is challenging. The implementation of scientific approaches may be efficacious in achieving changes in practice. This study aims to improve the nutrition-related care of older surgical patients using an action research approach. The first phase (observe) involved a quantitative clinical audit of the nutrition-related care provided to patients $\geq 65$ years admitted to general surgical wards, followed by the reflect phase, which used semi-structured qualitative interviews with clinicians selected using maximum variation purposive sampling. Qualitative data were analysed using framework analysis. A clinical audit $(n=75)$ identified practice gaps in the nutritional care of older surgical patients (median 72 years (range 65-95), 61\% male). Only $17 \%(\mathrm{n}=13)$ and $4 \%(\mathrm{n}=3)$ of patients met fasting targets of $2-4 \mathrm{~h}$ for fluid and $6-8 \mathrm{~h}$ for food respectively, and no patient received preoperative carbohydrate loading. Fifty-five percent $(n=41)$ of patients were upgraded to the full ward diet by postoperative day one. Interviews with 12 clinicians identified three major barriers to practice change: (a) the complexity of the context (e.g., unpredictable theatre times, requirement for flexibility); (b) strong surgeon-led decision making hierarchy; and (c) poor communication and teamwork (within and between disciplines). Insight gained into the barriers to practicing change will aid in the development of effective and targeted intervention strategies (the plan and act phases) to achieve the sustainable translation of evidencebased best practice into clinical practice.

\subsection{Digestibility of Riceberry Rice Flour with Different Thermal Treatments and Particle Size Distributions}

\footnotetext{
L. Thiranusornkij ${ }^{1}$, S. Adisakwattana ${ }^{2}$, A. Chandrachai ${ }^{1}$, D. Kuakpetoon ${ }^{3}$ and P. Thamnarathip ${ }^{4}$

1 Technopreneurship and Innovation Management Program, Graduate School, Chulalongkorn University, Thailand

2 Department of Nutrition and Dietetics, Faculty of Allied Health Sciences, Chulalongkorn University, Thailand

3 Department of Food Technology, Faculty of Science, Chulalongkorn University, Thailand

4 KCG Excellence Center, KCG Corporation Co., Ltd., Thailand
} 
(1) Background: Riceberry (Oryza sativa L.) is a dark-purple rice crossbred between Hom Mali 105 (HM) and Thai Hom Nil (HN). It has been reported as a potent source of antioxidants and dietary fiber. However, there have been no studies on the digestive ability of riceberry and its effects of particle size and thermal processing methods. (2) Methods: This study evaluated the effect of thermal treatment (non-heating; NRB, tray-drying; TRB, and dried-boiled riceberry rice flour; $\mathrm{BRB}$ ), with various particle size distributions (<90, 90-125, 125-180, 180-250, and 250-355 m) of riceberry rice flour (RF) on the in vitro digestive properties. (3) Results: It seemed that thermal treatment produced a smaller size of RF at the same range of particle size distribution. The increased particle size of the flour was associated with a reduced digestive ability, including total starch (TS), slowly digestible starch (SDS) and estimated glycemic index (eGI) $(p<0.05)$, while the thermal processing method showed no significant effect. (4) Conclusion: The results indicate that the particle size of RF is able to control starch digestibility.

\subsection{Influence of Platescapes and Foodscapes on Dietary Intake among Government Employees in Malaysia}

\section{Y. T. Er and R. Jamaluddin}

Department of Nutrition and Dietetics, Faculty of Medicine and Health Sciences, Universiti Putra Malaysia, Selangor 43400, Malaysia

In built environments, elements in the microscale environment such as platescapes (the appearance of a food container) and foodscapes (the appearance of food) have become a concern, as they may influence a person's energy intake. This quasi-experimental study assessed the influence of platescapes and foodscapes on the dietary intake of respondents. A total of 133 respondents (54 males, 79 females), mean age $36.83 \pm 7.26$ years participated in this study. The respondents completed a self-administered questionnaire on sociodemography, platescape and foodscape preference. For platescapes, a plate mapping method was used, where respondents placed various sizes of food models on different sized plates (9-inch and 11-inch) based on their preferences. For foodscape preference, the respondents placed various sized food models differentiated by shapes and colours on a nine-inch plate. Two-day, 24-h dietary recall was obtained by interview. The average energy intake of the respondents was $1741 \pm 339 \mathrm{kcal}$ (males) and $1625 \pm 247 \mathrm{kcal}$ (females). Significant differences were found between sex, age and platescapes with energy intake $(p<0.05)$. An 11-inch plate $(561 \pm 143 \mathrm{kcal})$ resulted in significantly higher energy intake $(p<0.05)$ than a 9-inch plate $(419$ $\pm 124 \mathrm{kcal}),(p>0.05)$. There were significant differences and associations between white rice and multicoloured rice, unicoloured and multicoloured protein and unicoloured and multicoloured vegetables with energy intake $(p<0.05)$. Moreover, significant difference were also found between circular and cube-shaped protein preferences. Only the shape of the carrots in the vegetables was significantly associated with energy intake. Subconsciously, these elements (bigger plate, multicoloured and circular shaped food) increased an individual's energy intake, and should thus be considered when assessing one's dietary intake.

\subsection{The Whole Grain Gap: Comparing Intakes to Recommendations}

\section{R. Suthers, R. Williams, M. Broom, A. Locke, S. Grafenauer and E. Beck}

1 Grains \& Legumes Nutrition Council, North Sydney, NSW, Australia

2 University of Wollongong, Wollongong, NSW, Australia

The $48 \mathrm{~g}$ whole grain daily target intake (DTI) is based on evidence of a reduced risk of highly prevalent chronic diseases such as heart disease and type 2 diabetes. The aim of this study was to assess the proportion of adults meeting the whole grain DTI in a nationally representative sample of Australians aged $15-70$ years $(n=1221)$. Participants completed a two-day food record and an attitudinal survey. The median daily whole grain intake was $26 \mathrm{~g}$ (IQR $4-58 \mathrm{~g}$ ). Only 31\% of adults met the $48 \mathrm{~g}$ DTI, consuming at least three serves of foods that were high in whole grain ( $16 \mathrm{~g} / \mathrm{serve})$. This is equivalent to a bowl of whole grain breakfast cereal and a sandwich with whole grain bread. Of those who fell short, 55\% consumed less than two serves ( $<32 \mathrm{~g}), 38 \%$ consumed less than one 
serve $(<16 \mathrm{~g})$ and $14 \%$ were non-consumers. The most common barrier to intake was a preference for refined grain foods. Participants' listed that competitive pricing, clear on-pack labelling and information about health benefits were facilitators of whole grain consumption. This study supports existing research that shows that the majority of Australians are not meeting the whole grain recommendations associated with health and reduced chronic disease risks. Clear messaging by the food industry about whole grain foods and improved education by healthcare professionals is required to encourage Australians to choose whole grain foods more often.

\subsection{High-Amylose Wheat Lowers the Glycemic and Insulinemic Response of Bread in Healthy Adults}

D. P. Belobrajdic ${ }^{1}$, A. Regina ${ }^{2}$, B. Klingner ${ }^{1}$ and A. R. Bird ${ }^{1}$

1 CSIRO Health and Biosecurity, Adelaide 5000, Australia

2 CSIRO Food and Agriculture, Canberra 2601, Australia

Conventional wheat foods contain high levels of readily digestible starch that commonly gives these foods a high glycemic index/response and may contribute to the development of type- 2 diabetes and cardiovascular disease. It was the aim of this study to determine whether a newly developed variety of wheat containing an elevated level of starch that resists digestion in the small intestine dampens postprandial glycemia. On separate mornings, 20 healthy non-diabetic men and women consumed four different wholemeal or refined wheat breads made from conventional or high amylose wheat flour (matched serving size of $121 \mathrm{~g}$ ). Venous blood samples were collected at regular intervals before and for three hours after the breakfast meal. High amylose wheat breads had lower glycemic, insulinemic and incretin responses than conventional wheat breads $(p<0.05)$. The processing of the flour (wholemeal or refined) did not affect the glycemic or other metabolic endpoints measured. The amylose and resistant starch contents of the breads did not influence plasma ghrelin, which suggests that satiety was unlikely to have been affected by consumption of the different meals. In conclusion, these findings demonstrate that replacing conventional with high amylose wheat flour is an effective strategy for lowering postprandial glycemic and insulinemic responses to bread.

\subsection{Chemical and Physical Changes in Oils during Heating}

F. De Alzaa, C. Guillame and L. Ravetti

Modern Olives, Lara, Victoria, Australia, 3212 and Boundary Bend Olives, Boundary Bend, Victoria 3599, Australia

When cooking oils are heated, oil degradation occurs, and by-products are produced (free fatty acids (FFAs), secondary products of oxidation, polar compounds). Some of these by-products have adverse effects on health. The smoke point of an oil is believed to be correlated with safety/stability under heat, although technical evidence is limited. This study assessed the correlation between an oil's smoke point and other chemical characteristics associated with stability/safety when cooking. Analysis was undertaken in an ISO17025 accredited laboratory. EVOO and other cooking oils were heated to $240{ }^{\circ} \mathrm{C}$ and exposed to $180^{\circ} \mathrm{C}$ for $6 \mathrm{~h}$, with samples assessed at various times, testing the smoke point, oxidative stability, FFAs, polar compounds, fatty acid profiles and antioxidant levels. EVOO yielded low levels of polar compounds and oxidative by-products, and maintained high levels of antioxidants, in contrast to the high levels of by-products generated for oils such as canola. The fatty acid profile and natural antioxidant content of EVOOs allowed the oil to remain stable when heated (unlike oils with high levels of poly-unsaturated fats (PUFAs), which degraded more readily). This study reveals that the smoke point does not predict oil performance when heated. Oxidative stability and secondary products of oxidation are better predictors together with the total level of PUFAs and antioxidants. Of all the oils tested, EVOO was shown to be the safest and healthiest to cook with. 
2.181. An Integrated Nutrition-Focused Food Security Approach to Address Childhood Malnutrition and Improve Maternal Outcomes in Vietnam

N. T. D. Hoang ${ }^{1}$, T. D. Le ${ }^{2}$, H. T. T. Cao ${ }^{2}$ and E. A. Szymlek-Gay ${ }^{1}$

1 Institute for Physical Activity and Nutrition (IPAN), School of Exercise and Nutrition Sciences, Deakin University, Burwood, VIC 3125, Australia

2 National Institute of Nutrition, Hanoi, Vietnam

Despite considerable improvements in maternal and child health in Vietnam, poor nutritionrelated health outcomes are still prevalent in some communities. A two-year community-based integrated nutrition-focused food security program was implemented in two remote communes in Lao Cai province in Vietnam between January 2015 and December 2016. The program's aim was to address the poor health and nutrition status of women of reproductive age and children under the age of five years. The program focused on increasing food availability for complementary feeding, maternal education (both practical and theoretical) related to breastfeeding, complementary feeding of infants, and young child nutrition, as well as nutrition education and maternal care during pregnancy. Anthropometric, dietary, knowledge/practice, and health indicators were measured at baseline and completion of the program. At the end of the program, underweight decreased in children from $24.2 \%$ at baseline to $16.6 \%$ at endline, and stunting decreased from $64.9 \%$ at baseline to $47.9 \%$ at endline. Maternal knowledge and implementation of breastfeeding and complementary feeding were significantly improved in most of the infant and young child feeding indicators defined by WHO/UNICEF 2010. Maternal health care improved with more women receiving antenatal care (65.8\% at baseline vs. $92.2 \%$ at endline), more women being immunized (62.4\% at baseline vs. $89.4 \%$ at endline), and fewer women giving birth at home without medical support (68.4\% at baseline vs. $47.8 \%$ at endline). An integrated nutrition-focused food security approach can reduce childhood malnutrition by addressing its multi-determinants through promoting and supporting optimal health and nutrition care practices among vulnerable women and children.

\subsection{Refined Carbohydrate vs. Saturated Fats: Effects on Cardiovascular Risk Profile}

K.-T. Teng ${ }^{1}$, L.-F. Chang ${ }^{2}$, S. R. Vethakkan ${ }^{2}$, K. Nesaretnam ${ }^{1}$ and T. A. B. Sanders ${ }^{3}$

1 Product Development and Advisory Services Division, Malaysian Palm Oil Board, 6, Persiaran Institusi, Bandar Baru Bangi, 43000 Kajang, Selangor, Malaysia

2 Department of Medicine, Faculty of Medicine, University of Malaya, Jalan Universiti, 50603 Kuala Lumpur, Malaysia

3 Diabetes and Nutritional Sciences Research Division, King's College London, Franklin-Wilkins Building, 150 Stamford Street, London SE1 9NH, UK

Modification of the amount and type of dietary fat has diverse effects on cardiovascular risk. We recruited 54 abdominally obese subjects to participate in a prospective cross-over design, single-blind trial comparing an isocaloric $2000 \mathrm{kcal}$ MUFA or carbohydrate-enriched diet with a SFA-enriched diet (control). A total of $\sim 7 \mathrm{E} \%$ of MUFA or refined carbohydrates were exchanged with SFA in the MUFA-rich and carbohydrate-rich diets, respectively, for six weeks. Blood samples were collected at fasting upon trial commencement and at week five and six of each dietary intervention phase to measure the levels of thrombogenic markers (E-selectin, PAI-1, D-dimer) and lipid subfractions. Radial pulse wave analysis and a 6-h postprandial mixed meal challenge were carried out in week six of each dietary intervention. No significant differences in fasting thrombogenic factors were noted between diets $(p>0.05)$. The MUFA meal elevated plasma D-dimer postprandially compared with the SAFA meal $(p<0.05)$. Comparing the three meals, there were similar postprandial elevations in D-dimer and postprandial reductions in PAI-1, augmentation index and pressure (time effect: $p<$ 0.05). The CARB diet was found to reduce HDL3 by $7.8 \%$ and increase small dense HDL (sdHDL) by $8.6 \%$ compared with the SFA diet $(p<0.05)$. The SFA diet increased the large HDL subfractions compared with both the CARB and MUFA diets by $4.9 \%$ and $6.6 \%(p<0.05)$, respectively. Overall, the evidence presented in this study suggests that the replacement of SFA with MUFA or refined 
carbohydrates may not improve thrombogenic markers in abdominally overweight individuals. Indeed, increased refined carbohydrate consumption adversely impacted fasting HDL subfractions.

\title{
2.184. Discretionary Intake among Indigenous Australian Adolescents and Its Relationship with Sex, Body
} Image, Size and Remoteness

\author{
F. Fayet-Moore ${ }^{1}$, A. McConnell ${ }^{1}$, J. Kim ${ }^{2}$ and P. Petocz ${ }^{3}$ \\ 1 Nutrition Research Australia, Sydney, NSW 2000, Australia \\ 2 Nestlé Australia Ltd., Rhodes, NSW 2138, Australia \\ 3 Department of Statistics, Macquarie University, North Ryde, NSW 2109, Australia
}

Twenty-six percent of the burden of disease among the Australian Indigenous population can be reduced by eliminating poor diet, alcohol and high body mass. Adolescents have the poorest diets and a higher prevalence of body image issues. We investigated the relationship between discretionary food intake among adolescents and demographic, geographic, anthropometric and body image variables. Day 1 data from the 2012-13 National Aboriginal and Torres Strait Islander Nutrition and Physical Activity Survey for adolescents $12-18$ y were analysed $(n=588)$. Discretionary foods (DF) are high in saturated fats, added sugars, and/or salt. Waist circumference to height ratio classified participants' risk of metabolic complications. Remoteness, body image, and DF energy contribution were analysed. The prevalence of being at increased risk of metabolic complications was higher among boys $(42.6 \%)$ compared to girls $(28.2 \%)(p<0.05)$, and $68 \%$ of at-risk boys perceived themselves as overweight, compared to $36 \%$ of girls. DF contributed $39.9 \%$ of total energy, with no difference between boys $(40.9 \%)$ and girls $(38.9 \%)$, but was lower among those from remote $(33.5 \%)$ rather than non-remote $(41.5 \%)$ areas $(p<0.05)$. The energy contribution from DF was higher among boys who perceived themselves as being overweight (56.3\%) than girls (26.9\%). Boys were more likely to be at risk of metabolic complications, and at-risk boys were more likely to perceive themselves as overweight. DF intake was lower in remote areas and among girls who perceived themselves as overweight. These results support a need to reduce DF intake among Indigenous adolescents and implement gender-specific strategies related to body image, taking remoteness of location into account.

\subsection{The Indigenous Australian Malnutrition Project: The Burden and Impact of Malnutrition in Regional} Hospitals

N. F. Morris ${ }^{1,2}$, S. Stewart ${ }^{1,3}$, M. Riley ${ }^{4}$ and G. P. Maguire ${ }^{1,2}$

1 Monash University, Clayton, Victoria 3800, Australia

2 Baker Heart and Diabetes Institute, Melbourne 3004, Australia

3 Australian Catholic University: Mary MacKillop Institute for Health Research, Melbourne 3000, Australia

4 CSIRO Health and Biosecurity, Adelaide 5000, Australia

Malnutrition is a recognised health problem in metropolitan but not regional hospitals. The aims of this study were to determine the burden and impact of malnutrition in Indigenous and nonIndigenous Australian inpatients in regional Australian hospitals. The Subjective Global Assessment tool was used to assess nutrition status in adult medical inpatients. Malnutrition-related outcomes were determined by length of hospital stay, discharge destination, 30-day and six-month hospital readmission and six-month mortality. Of 608 patients enrolled, 250 (41.1\%) were malnourished. Indigenous Australians were more likely to be malnourished than non-Indigenous patients (OR 1.45, $95 \%$ CI 1.03-2.04, $p=0.024$ ). The length of hospital stay was significantly longer in malnourished patients (median difference $=2$ days, $p<0.001$ ). Malnourished patients were also more likely to be readmitted to hospital within 30 days (OR 2.15, 95\% CI 1.45-3.20, $p=0.001$ ) and six months (OR 1.97, $95 \%$ CI 1.39-2.79, $p=0.001$ ). Higher rates of mortality at 30 days (RR 1.95, 95\% CI 1.51-2.50, $p=0.001$ ) and at six months (RR 1.78, 95\% CI 1.45-2.18, $p<0.001$ ) were observed in malnourished patients. We found higher rates of malnutrition than previously reported in Australia and malnourished patients had higher rates of health care utilization and mortality than nourished patients. 


\subsection{Effect of Polysorbate 80, a Commonly-Used Emulsifier, on Serum Lipids and Organ Histology: In} Vivo Rat Model Study

S. Zulaiha, E. P. Peter, A. Selvaretnam, C. S. Lim, C. E. Nahar, G. Dass, R. Valsala, S. Shyam, K. L. Ho and P. Krishnappa

International Medical University, Kuala Lumpur Malaysia

(1) Background: Polysorbate 80 (P80) is a commonly-used emulsifier and is generally regarded as safe. While the acceptable daily intake of $P 80$ is $10 \mathrm{mg} / \mathrm{kg}$ body weight, human consumption grossly exceeds this limit. Emerging evidence suggests that P80 can cause metabolic dysfunction. We investigated the effect of P80 on serum lipids and organ histology in an in vivo rat model. (2) Methods: Sprague Dawley rats $(n=24)$ were randomised into four equal groups. For four weeks, treatment groups were administered a daily oral gavage of P80 $(0.5 \%(250 \mathrm{mg} / \mathrm{Kg} \mathrm{BW}), 1 \%(500$ $\mathrm{mg} / \mathrm{Kg} \mathrm{BW})$ or $2 \%(1000 \mathrm{mg} / \mathrm{Kg} \mathrm{BW})$, and the control group was administered distilled water. The body weight, food and water intake were monitored daily. After four weeks, blood was collected, the rats were sacrificed and the organs harvested. Liver and colon specimens were stained with haemotoxylin and eosin and evaluated using light microscopy. (3) Results: Though the food and water intakes were not significantly different between the groups, a trend towards higher weight gain was seen among the P80 groups. Rats on 1\% P80 had a significant increase in total HDLcholesterol ratio $(p<0.01)$. The liver specimens showed a dose-related increase in steatosis and inflammation, with 2\% P80 showing ballooned hepatocytes. The colon of rats fed 1\% P80 showed necrosis, inflammation, crypt abscess, ulcers and a reduced mucus lining. These histological changes were absent in the control group. (4) Conclusion: P80 shows significant hyperlipidaemic effects and aberrations in the organ histology of rats. While the P80 exposures in this study were high, the results warrant a need for the careful evaluation of exposure to large doses of P80 over time.

\subsection{Claiming Health: What Effect Has the New Nutrition and Health Claims Standard Had on Food Labels?}

D. L. Alexander ${ }^{1}$ and J. A. North ${ }^{2}$

1 Ministry for Primary Industries, New Zealand

2 Foodcom consultant, New Zealand

In 2013, New Zealand and Australia adopted the Food Standards Code Standard 1.2.7 Nutrition, health and related claims on foods which came into full effect in January 2016. In 2014/15, a New Zealand baseline survey of such claims on food labels was undertaken, and this was recently repeated to quantify the changes made due to the implementation of the new standard. A random and representative sample of 640 packaged foods available in New Zealand supermarkets across 16 different categories was assessed for both nutrition content claims and health (general level and high level) claims on packaging. In some food categories, up to $79 \%$ of randomly-selected products made some form of nutrition or health claim. Nutrition content claims were most common in the following categories: cereals and cereal products $(79 \%)$, snack foods $(79 \%)$ and edible oils $(75 \%)$. The most common nutrition content claim was "gluten free". General level health claims appeared less frequently, but mostly in the special purpose food (16\%), bread and bakery product $(6 \%)$ and packaged fruit and vegetable (6\%) categories. High level health claims were infrequently used and only found on cereals and cereal products $(2 \%)$ and packaged fruits and vegetables (1\%). The main difference since the baseline survey in 2014/15 was the increase in nutrition content claims across all categories of foods. This indicates an increased interest amongst manufacturers, potentially led by consumer demand, in communicating the nutritional benefits of foods; one of the reasons New Zealand and Australia established the robust regulatory framework for such claims.

2.188. Does Using the Health Star Rating Come at a Cost? A Price Comparison of Similar Products That Do and Do Not Display the HSR

M. de Abreu ${ }^{1,2}$, K. Charlton ${ }^{2}$, Y. Probst ${ }^{2}$, N. Li $^{1}$, M. Crino ${ }^{1}$, B. Neal ${ }^{1}$ and J. H. Y. Wu ${ }^{1}$ 
1 The George Institute for Global Health, and the University of New South Wales, Newtown, New South Wales, Australia

2 School of Medicine, University of Wollongong, New South Wales, Australia

The Health Star Rating (HSR) is a front-of-pack label designed to help consumers identify healthier packaged foods. To investigate whether consumers are paying a price premium to use the HSR to guide food choices, this study investigated the association between the presence of the HSR and the price of breakfast cereals, cereal-based bars, and juices. The prices of packaged foods were collected from shopping receipts provided by the participants of an Australian food label trial (June 2014 to June 2016). Nutrient data were obtained from the Nutrient Information Panel, and the HSR calculated according to government criteria. The association between the presence of the HSR and the price per energy $(\$ / 100 \mathrm{~kJ})$ and unit cost $(\$ / 100 \mathrm{~g})$ of similar packaged food products were assessed by linear regression. Of the products investigated, $50 \%(n=87)$ of breakfast cereal, $20 \%(n=20)$ of cereal-based bars, and 7\% $(n=10)$ of juice products displayed the HSR. The average package size was not significantly different between products that displayed the HSR and products that did not across the three product categories ( $p \geq 0.31$ for each). For all product categories, no significant difference in price per unit or energy cost was observed ( $p \geq 0.16$ for each). For example, the mean difference (95\% CI) between products that displayed the HSR and products that did not for breakfast cereals was $\$ 0.07(-0.07,0.22)$ per $100 \mathrm{~g}$ and $\$ 0.01(-0.01,0.02)$ per $100 \mathrm{~kJ}$. This study found no significant price difference according to whether products displayed the HSR or not. This suggests that consumers are not being charged a price premium in order to use the HSR to guide their packaged food choices.

\subsection{Who Is Learning about "Clean Eating" Diets or Learning from Nutrition Professionals on Social Media? \\ K. Klassen ${ }^{1}$, M. M. Adamski ${ }^{1}$, L. Brennan ${ }^{2}$, M. Reid ${ }^{2}$, M. Lim ${ }^{3}$, T. A. McCaffrey ${ }^{1}$ and H. Truby ${ }^{1}$ \\ 1 Department of Nutrition, Dietetics and Food, Monash University, Melbourne 3800, Australia \\ 2 School}

There has been growing concern about the use of social media (SM) to promote non-evidencebased diet trends like 'clean eating' in place of qualified nutrition advice. The aim of this study was to explore the characteristics of individuals using SM to learn about nutrition-related topics and to compare and contrast these characteristics between two distinct topics: (1) learning about cleaneating or detox diets (CEDD); and (2) learning from nutrition professionals (NP). Learners from a three-week online course "Food as Medicine" completed a survey. Multivariable logistic regression models were constructed to evaluate the characteristics associated with learning from social media: (1) about CEDD; and (2) from NP. A total of 1005 learners from 70 countries completed the survey; the mean age was 50 years (s.d. 15). CEDD information-seeking was negatively associated with trusting information from healthcare professionals OR 0.64 (95\% CI 0.52 to 0.79 ), and positively associated with trusting social media posts by friends OR 1.3 (95\% CI 1.02 to 1.71), and reading social media posts of strangers more than friends OR 1.34 (95\% CI 1.09 to 1.66). NP information-seeking was negatively associated with trusting media OR 0.70 ( $95 \%$ CI 0.56 to 0.90$)$. There were differences between people accessing information about CEDD or from NPs, including trusting various information sources and social media user profiles. Nutrition professionals should try to understand the different user profiles to ensure that public health nutrition messages are effectively targeted.

\subsection{Fruits and Vegetables and Olive Oil Do Not Preserve Skeletal Muscle in Obese Older Adults during Energy Intake Restriction}

A. M. Villani ${ }^{1}$, H. Wrightt ${ }^{1}$, G. Slater ${ }^{1}$ and J. Buckley ${ }^{2}$

1 University of the Sunshine Coast, Queensland Australia

2 ARENA, Sansom Institute for Health Research, University of South Australia, Adelaide, Australia

Energy-restricted weight loss diets in older adults results in the loss of skeletal muscle mass (SMM), which may predispose to disability. A high protein intake may attenuate the loss of SMM. 
Fruit and vegetables (F\&V) and extra-virgin olive oil (EVOO) are rich sources of carotenoids and polyphenols, both of which are inversely associated with sarcopenic symptomology. This study investigated the effect of an increased intake of carotenoid-rich F\&V and EVOO against a background high protein diet on body composition, strength and physical function in obese older adults during dietary energy-restricted weight loss. Sixty-five obese older adults (68.7 \pm 5.6 yrs; BMI: $33.7 \pm 4.8$ $\mathrm{kg} / \mathrm{m}^{2}$ ) were randomized to a 12-week energy-restricted, isocaloric diet (30\% protein $(1.2 \mathrm{~g} / \mathrm{kg}) ; 30 \%$ carbohydrate; $40 \%$ fat) enriched with either: (a) high carotenoid F\&V, and EVOO (HC + EVOO); or (b) lower carotenoid F\&V, and a polyunsaturated based oil (LC + PUFA). At baseline and postintervention, body mass, body composition (DXA), strength (hand-grip strength) and physical performance (Short Physical Performance Battery) were assessed. Body mass reduced similarly in both groups (HC+EVOO: $-6.5 \mathrm{~kg}$; 95\% CI: $-7.7,-5.3 \mathrm{~kg}$; LC+PUFA: $-7.2 \mathrm{~kg}$; $95 \%$ CI: $-9.1,-5.5 \mathrm{~kg} ; p=$ 0.79). SMM also decreased, with no difference between groups (HC+EVOO: $-1.7 \pm 1.3 \mathrm{~kg}$; LC+PUFA: $-1.8 \pm 1.4 \mathrm{~kg} ; p=0.92)$. No significant change in muscle strength was observed, with no difference between groups $(p=0.91)$. Small, non-significant improvements were observed in physical function, with no difference between groups $(p=0.40)$. High carotenoid F\&V plus EVOO do not preserve SMM during energy intake restriction in obese older adults.

\subsection{Monosodium Glutamate Intake and Mortality in Chinese Adults: A 10-Year Follow-Up Study}

\section{Z. Shi' ${ }^{1}$ S. Zhen'², Y. Zhou' ${ }^{2}$ and M. Riley ${ }^{3}$}

1 Adelaide Medical School, University of Adelaide, Australia

2 Jiangsu Provincial Center for Disease Control and Prevention, China

3 Commonwealth Scientific and Industrial Research Organisation (CSIRO), Australia

No population study has assessed the association between monosodium glutamate (MSG) intake and mortality. We aimed to assess whether MSG and total glutamate intake was associated with cardiovascular disease (CVD) mortality, cancer mortality, and all-cause mortality in a prospective cohort of the Chinese population. We studied 2832 adults aged 20 years and above with a mean follow-up of 10 years. MSG intake was quantitatively assessed in 2002. Hazard ratios (HRs) and $95 \%$ confidence intervals were calculated by competing risks regression (CVD and cancer mortality) and Cox proportional hazards analysis (all-cause mortality). We documented 184 deaths (including 70 CVD deaths and 63 cancer deaths) during 27,742 person-years of follow-up. No association between MSG intake and all-cause mortality was found. After adjusting for sociodemographic and lifestyle factors as well as energy and fat intake, across quartiles of MSG intake, the HRs for all-cause mortality were 1.00, 1.09 (0.68-1.76), 1.49 (0.94-2.37), and $0.92(0.57-1.51)$, respectively. HRs for all-cause mortality across quartiles of total glutamate intake were $1.00,0.84(95 \%$ CI 0.56-1.26), 0.73 (95\% CI 0.46-1.16), and 0.56 (95\% CI 0.32-0.98) ( $p$ for trend 0.038), respectively. However, the inverse association between total glutamate intake and all-cause mortality became nonsignificant after further adjusting for overall dietary patterns. MSG intake was inversely associated while total glutamate intake was positively associated with vegetable rich dietary pattern. In conclusion, there was no association between MSG intake and all-cause mortality. Meanwhile, total glutamate intake was inversely associated with all-cause mortality, which may be due to an overall healthy dietary pattern.

\subsection{The Longitudinal Association between Diet and Pregnancy Status among Australian Women of} Reproductive Age

E. Gresham ${ }^{1,2}$, L. Leigh ${ }^{3}$, C. Oldmeadow ${ }^{3}$, C. Collins ${ }^{4}$, J. Byles ${ }^{5}$ and A. Hure ${ }^{5}$

1 School of Allied Health Science, Griffith University, QLD, Australia

2 Health Intelligence Unit, Western New South Wales Health, NSW, Australia

3 Clinical Research Design and Statistics Support (CReDITSS) Unit, and Hunter Medical Research Institute (HMRI), NSW, Australia

4 School of Health Sciences, and Priority Research Centre in Physical Activity and Nutrition, Faculty of Health \& Medicine, University of Newcastle, NSW, Australia 
5 School of Medicine and Public Health, University of Newcastle, and HMRI, NSW, Australia

Maternal diet quality has been associated with several favourable pregnancy and birth outcomes. The aim of this study was to investigate the relationship between change in diet quality and pregnancy status among women of reproductive age. Self-reported data were collected prospectively for the Australian Longitudinal Study on Women's Health (ALSWH) from women born between 1973 and 1978. The Dietary Questionnaire for Epidemiological Studies in 2003 (Survey 3) and 2009 (Survey 5) was used to calculate diet quality using the Australian Recommended Food Score (ARFS) modified for pregnancy. A sub-sample of 6883 women, aged 20 to 25 years in 2003 and 31 to 36 years in 2009, were classified as preconception, pregnant, and not pregnant (with or without previous children). Regression analyses showed that changes in pregnancy status were significantly associated with changes in total ARFS $(p<0.01)$. Pregnancy status change from not pregnant (with no previous children) to pregnant was associated with the greatest overall change in ARFS total (1.9 unit increase out of a maximum of 72), which remained significant after adjustment for changes in potential confounders. Diet quality increases as women age and with pregnancy; however, there remains a wide scope for further improvements.

\subsection{Umami Application Improves Appetite and Well-Being among the Elderly: A Community-Based Pilot Project \\ Misra Snigdha ${ }^{1}$, Lau Chin Mun ${ }^{2}$ and Purple Chiah Ping Yan ${ }^{2}$ \\ 1 Department of Nutrition \& Dietetics, International Medical University, Jalan Jalil Perkasa 19, Bukit Jalil, 57000 Kuala Lumpur, Malaysia \\ 2 Corporate Communications Department, Ajinomoto (Malaysia) Berhad, Kuala Lumpur, Malaysia}

The elderly are vulnerable to malnutrition for many reasons, a factor that affects their nutritional status. With an increase in the ageing population, it is imperative to identify sustainable preventive measures to promote healthy ageing with good quality of life. In view of the preventive health needs of senior citizens, a community health programme titled "Eat Well, Live Well Together with Umami" was designed. This programme aimed to promote a balanced and low-sodium diet for the elderly through Umami applications. Information on appetite behaviour, dietary intake, observed plate wastage, and perception of the meals consumed were obtained. Anthropometrics to determine participants' body weight status, fasting blood sugar, lipid profile, and blood pressure were also assessed. Urinary sodium was analysed using spot urine samples to determine the level of sodium excretion. The menu was modified to provide healthy and balanced meals, with sodium adjusted to recommended levels. The study participants included 55 elderly residents, who were over 60 years of age. An improvement of appetite was observed in $31.3 \%$ of the residents before menu modification and in $81.3 \%$ after menu modification. The sodium content of the modified menu was reduced by $14 \%$ in a single meal. Most (64.3\%) of the residents were reported to have health problems (hypertension, diabetes, hypercholesterolemia). However, all the above parameters showed encouraging results after introducing the modified menu. Blood pressure was reduced among 50\%, without the use of hypertensive medications. This pilot project indicated that a small and sustainable change in the diet of the elderly can augment their quality of life.

\subsection{Dietary Calcium Intake Status among Chinese Elderly over 60 Years of Age during 2010-2012}

\section{Z. Liu, S. J. Pang, P. K. Song, S. S. Jia and J. Zhang}

National Institute for Nutrition and Health, Chinese Center for Disease Control and Prevention, Beijing 100050, China

Inadequate calcium is one of the most common nutrient deficiencies in China. Moreover, little is known about the status of dietary calcium intake in the elderly, that is, those over 60 years of age. We aimed to learn the status of dietary calcium intake among Chinese elderly aged 60 years old and above during the period of 2010-2012. Using dietary data of the 24-h recall method for three consecutive days and the food weighting method from the Chinese Nutrition and Health Surveillance 
in 2010-2012, dietary calcium intake and its resources were calculated, analyzed, and compared for the elderly, taking into account different areas of residence, gender, and age. The dietary calcium intake of Chinese elderly over 60 years of age was $344.20 \mathrm{mg} /$ day, and the ratio was only $1.19 \%$ of calcium intake over the RNI (Recommended Nutrient Intake). The dietary calcium intake was significantly higher in the urban elderly population than that in the rural elderly group. The dietary calcium intake was also significantly higher in the elderly aged 60-69 years than that in the elderly aged over 70 years. The main food sources of dietary calcium were vegetables $(33 \%)$ and cereals $(22 \%)$; meanwhile, the proportion of calcium obtained from milk was lower than $7 \%$. Compared with the calcium intake in 2002, the dietary calcium intake of the elderly obviously decreased during the period of 2010-2012, amounting to about $0.4 \sim 22.8 \%$. Dietary calcium intake is seriously inadequate in the Chinese elderly aged 60 years and above. Furthermore, it exhibits a descending trend.

\subsection{Perceptions of Access to Food in Older Adults}

A. McMahon, R. Crowe, S. Evans and J. Russell

1 School of Medicine, University of Wollongong, NSW, Australia

2 School of Health and Society, University of Wollongong, NSW, Australia

Evidence to date on food insecurity in Australia is limited to findings based on only one question, providing an indication of the risk of food insecurity rather than a measure. This single question underestimates the extent of food security issues as it only covers economic access to food. A number of experience-based tools exist to measure food insecurity at the household and individual levels, but these are not appropriate for use in older adult populations. This study is a formative phase that uses qualitative research methods to develop a quantitative survey tool to assess access to food in older adults that is relevant to their stage of life and health outcomes. Senior citizen clubs and the University of the Third Age in the Wollongong area of New South Wales were approached to recruit adults aged 65 years and over to participate in focus groups lasting 45-60 min. Each focus group was digitally recorded and transcribed verbatim. Seven focus groups were conducted, each comprising four to 10 older adults. The majority of participants were women who lived alone. Themes emerging from analysis of the transcripts, include the importance of eating out and shopping trips for social interaction; the importance of budgeting due to living on a pension; and ergonomic issues with opening packets and jars. A quantitative survey to assess food security status in older adults will require a range of items that include physical and social items in addition to the economic issues of accessing food.

\subsection{The Relationship between Caffeine and Sleep Quality in Middle-Aged to Older Australian Adults}

V. C. Polvere ${ }^{1}$, E. Watson ${ }^{1}$, S. Banks ${ }^{1}$, A. M. Hill ${ }^{2}$, C. Yandell ${ }^{2}$, J. D. Buckley ${ }^{2}$, A. Scholey ${ }^{3}$ and A. M. Coates 2

1 Centre for Sleep Research, School of Psychology, Social Work \& Social Policy University of South Australia, Australia

2 Alliance for Research in Exercise, Nutrition and Activity, Sansom Institute for Health Research, School of Health Sciences, University of South Australia, Australia

3 Centre for Human Psychopharmacology, Swinburne University, Australia

Caffeine is a commonly consumed stimulant. The relationship between caffeine consumption and sleep quality was examined at baseline as part of a larger 12-week dietary intervention looking at diet and cognition. Adults aged between 50 and 80 years $(n=101: 56$ F, $62.8 \pm 7.9$ years, BMI $30.5 \pm$ $3.6 \mathrm{~kg} / \mathrm{m}^{2} ; 45 \mathrm{M}, 66.5 \pm 7.9$ years, BMI $29.8 \pm 3.2 \mathrm{~kg} / \mathrm{m}^{2}$ ) completed a validated caffeine food frequency questionnaire estimating total daily dietary caffeine (mg/day) consumption, as well as the Pittsburgh Sleep Quality Index (PSQI) for subjective sleep quality. PSQI scores below 5 indicate good quality sleep. Females consumed $271 \pm 217 \mathrm{mg}$ of caffeine per day and had PSQI scores of 5.9 \pm 3.0 , whereas males consumed $290 \pm 181 \mathrm{mg}$ per day and had PSQI scores of $5.8 \pm 4.0$, with no sex differences observed. Spearman rank correlations showed no relationship between caffeine intake and sleep 
quality (rho $=0.169, p=0.092$ ) or age and sleep quality (rho $=-0.056, p=0.581$ ). There were no differences in age between good $(\mathrm{n}=39)$ and poor $(\mathrm{n}=62)$ quality sleepers $(p=0.441)$; however, irrespective of age, those consuming high amounts of caffeine $(>400 \mathrm{mg} /$ day, $\mathrm{n}=19)$ had worse sleep quality compared with low ( $<400 \mathrm{mg} /$ day, $\mathrm{n}=82)$ caffeine consumers $(7.5 \pm 3.5$ vs. $5.5 \pm 3.1, p=0.03)$. These results suggest that high caffeine intake is associated with poor sleep quality and support the need for caffeine guidelines to be implemented in Australia to educate consumers about the relationship between caffeine and sleep quality.

Funding source: Almond Board of California

\subsection{Vitamin A Intake in Older New Zealand Adults}

C. Mackley and M. A. Choukri

Department of Nursing, Midwifery, and Allied Heath, Ara Institute of Canterbury, Christchurch, New Zealand

New Zealand as a nation is aging, with those aged 65 years or older expected to double by 2035. Several functions that are commonly affected by age-related health decline, such as vision problems, respiratory tract issues, and gut health, require optimal intake of vitamin A. However, there is little information about the dietary intake of vitamin A in this population. A total of 46 men and women aged 50 years or over completed a semi-quantitative FFQ developed to assess the intake of vitamin A-rich foods. Participants were ranked on their vitamin A intake, expressed as retinol equivalents per day, and compared between those aged 50-64 years and 65 years and over. No differences were observed by age, and all participants met or exceeded the RDI for vitamin A. The majority of intake came from plant-based foods, with carrots, pumpkin, and silverbeet being the highest contributing foods. The main animal-based sources included lamb liver, milk, and yoghurt. Sources of vitamin A established in this study are comparable to those found in the 2008/2009 New Zealand Adult Nutrition Survey. Interestingly, carrots provided about 4-5-fold of retinol equivalents compared to any of the other foods. The results indicate that while the current vitamin A intake in older adults is sufficient, additional information may need to be provided for this age group to increase intake from a greater variety of foods.

\subsection{Younger and Older Men Show Increased Total Energy Intake When Carbohydrates and Fat Are Added to a Protein Supplement \\ C. Giezenaar, I. Chapman, M. Horowitz and S. Soenen \\ NHMRC Centre of Research Excellence in Translating Nutritional Science to Good Health, Discipline of Medicine, Royal Adelaide Hospital, The University of Adelaide, Australia}

Protein-rich supplements are used widely to manage undernutrition in older people. Previously, we showed that the suppression of ad libitum energy intake by 'pure' whey-protein drinks was less in older than younger men, increasing total (drink + meal) energy intake in the older group. The aim of the study was to determine the effects of the addition of carbohydrates and fat to, or substitution of carbohydrates and fat for, protein, compared to 'pure' protein and a non-caloric control on energy intake in younger and older men. In a randomised, double-blind order, the administration $(\sim 450 \mathrm{~mL})$ of drinks containing $14 \mathrm{~g}$ protein, $28 \mathrm{~g}$ carbohydrate, $14 \mathrm{~g}$ fat ( $280 \mathrm{kcal}$, 'MIXED LOW'); $70 \mathrm{~g}$ protein, $28 \mathrm{~g}$ carbohydrate, $14 \mathrm{~g}$ fat (504 kcal, 'MIXED HIGH'); $70 \mathrm{~g}$ whey-protein ( $280 \mathrm{kcal}$, 'PROTEIN'); or an iso-palatable control drink ( $\sim 0 \mathrm{kcal}$, 'CONTROL') were followed by a buffet-style meal (180 $\mathrm{min})$ in 13 younger ( $22 \pm 3$ years, $24 \pm 2 \mathrm{~kg} / \mathrm{m}^{2}$ ) and 13 body mass index (BMI)-matched older (74 \pm 6 years, $\left.26 \pm 2 \mathrm{~kg} / \mathrm{m}^{2}\right)$ men. The suppression of ad libitum energy intake was less in older than younger men after the PROTEIN but not the MIXED drinks, when compared to the CONTROL $(p=0.038)$. Total energy intake was higher after the caloric drinks compared to the CONTROL $(p<0.05)$, and higher after MIXED HIGH $(p<0.05)$, but not MIXED LOW $(p>0.05)$ compared to PROTEIN drinks. In conclusion, total energy intake increased by the addition, but not substitution, of carbohydrates and fat to protein. 


\section{Poster Presentations}

\subsection{Copper Supplementation and Serum Lipid Level: A Meta-Analysis of Randomized Controlled Trials}

Wang Jing, Pan Tian-Qi, Zhang Hong, Wang Shao-Kang and Sun Gui-Ju

School of Public Health, Southeast University, Nanjing 210009, China

(1) Background and Aims: Many studies have evaluated the effect of copper supplementation on serum lipid levels. Therefore, a meta-analysis was conducted of randomized controlled trials investigating whether the supplement of copper would have an impact on the blood lipid levels. (2) Methods: Studies were retrieved by searching CNKI, PubMed, and Web of Science (from inception to March 2017) using the following keywords: nutrient copper and lipid level. Each randomized controlled trial of nutrient copper influencing blood lipid levels was retrieved according to the requirements of systematic review, then the quality of the included studies was evaluated and the meta-analysis was subsequently performed. Dates from three trials representing 146 participants were examined using a random-effects model or fixed-effects model. (3) Results: Pooled mean net change in total cholesterol (95\% CI: -0.15 to 0.14 ) and low density lipoprotein (95\% CI: -0.52 to 0.13 ) for those treated with copper supplementation showed no significant difference compared to the control group, but the difference between the high density lipoprotein (95\% CI: 0.01 to 0.68 ) of the test group and the control group was statistically significant. (4) Conclusion: Copper intake has an effect on high density lipoprotein, but whether it has an effect on serum lipid levels is still unknown and requires further study.

\subsection{Assessment of Salt-Related Knowledge, Attitudes, and Behaviours in Parents Participating in the Digital Education to Limit Salt in the Home (DELISH) Program}

\section{Khokhar, C. A. Nowson, C. Margerison, A. Booth, K. Campbell, C. A. Grimes}

Institute for Physical Activity and Nutrition, School of Exercise and Nutrition Sciences, Deakin University, Victoria, Australia

Salt intake among Victorian children and adults remains in excess of recommendations. Improving knowledge, attitudes, and behaviours (KABs) in parents may be an important precursor for reducing salt intake in children. Therefore, the aim of this study was to determine whether there was a change in salt-related KABs in parents following participation in a five-week web-based DELISH program delivered to primary school children. Parents received weekly educational newsletters and completed a salt-related KABs questionnaire pre- and post-program delivery. McNemar's tests were performed to determine the change in KABs pre- and post-program delivery. A total of 73 parents, $63 \%$ female (mean age 41.0 (SD 6.8) years), completed both the pre- and postprogram questionnaires. The percentage of parents aware of daily salt intake recommendation increased from $40 \%$ to $74 \%(p<0.0001)$ and awareness of bread as the main source of salt increased from $42 \%$ to $95 \%(p<0.0001)$. Whilst there was a reduction in the proportion of parents indicating that sodium information on food labels was difficult to understand ( $52 \% \mathrm{vs} .39 \%, p<0.009)$, there was no increase in the proportion who could correctly decipher sodium content on the nutrition information panel of bread $(73 \%$ vs. $81 \%, p=0.238)$. There was a significant increase in the proportion of parents rarely/never adding salt at the table $(62 \%$ vs. $82 \%, p<0.002)$ and during cooking $(45 \%$ vs. $59 \%, p<0.031$ ). In conclusion, it is evident that participation in the DELISH program was successful in improving salt-related knowledge and self-reported behaviours of Victorian parents.

\subsection{Iron Trapped by Hepcidin Autocrine in Plaque Macrophages: New Insight into the Iron Hypothesis of Atherosclerosis}

Lin Xiao, Gang Luo, Xiaoping Guo, Jing Wang, Hongmei Zeng, Feng Zhou and Ping Yao *

Department of Nutrition and Food Hygiene, School of Public Health, Tongji Medical College, Huazhong University of Science and Technology, Wuhan 430030, China 
* Corresponding Author

Iron accumulation has frequently been found in atherosclerosis (AS) lesions, especially focally within macrophages and foam cells, but the exact mechanisms by which this occurs and the pathophysiological roles of iron in atherogenesis are still debated. In this study, we explored the potential mechanisms by which hepcidin induced iron retention in plaque macrophages and its role in the formation of foam cells, focusing on the unique but largely undisclosed autocrine manner of hepcidin in macrophages. ApoE knock-out mice were fed a high-fat diet (HFD) for 16 weeks. RAW264.7 macrophages transfected with or without hepcidin-shRNA adenovirus were treated with ox-LDL to explore the interactions of ox-LDL, autocrined-hepcidin, and trapped iron in the formation of foam cells. Immunofluorescence analysis showed that hepcidin was co-localized with ox-LDL, TLR4, and ferritin-L in plaque macrophages of HFD-treated ApoE KO mice. RAW264.7 incubated with ox-LDL $(50 \mu \mathrm{g} / \mathrm{mL})$ showed elevated hepcidin $(61 \%, p<0.001)$, TLR4 $(150 \%, p<0.001)$, and ferritin- $\mathrm{L} / \mathrm{H}(63 \%, 50 \% ; p<0.001)$, as well as increased intracellular Oil Red O staining areas and concentrations of total/free cholesterol $(55.9 \%, 45.5 \%$; $p<0.05)$. Moreover, ox-LDL-induced iron retention and lipid accumulation were aggravated by LPS or exogenous hepcidin, but blocked by TAK-242, an antagonist of TLR4 or hepcidin silence. TLR4-mediated ox-LDL uptake induced hepcidin autocrine in plaque macrophages, which in turn stimulated the expressions of TLR4 and NF- $\kappa B$. Iron retention in AS plaque macrophages, instead of presentational disorder in systematic iron metabolism, aggravates foam cell formation and plaque lesions. The vicious circle between hepcidin autocrine and TLR4/NF- $\mathrm{KB}$ activation may contribute to plaque iron retention, shedding a profound light on insight into the AS "iron hypothesis".

\title{
3.4. Lipid-Lowering Effects of Phytosterols and/or Curcumin in Hypercholesterolaemic Individuals: A Randomised Controlled Trial
}

\author{
J. J. A. Ferguson ${ }^{1}$, E. Stojanovski ${ }^{2}$, L. MacDonald-Wicks ${ }^{3}$ and M. L. Garg ${ }^{1}$ \\ 1 Nutraceuticals Research Program, School of Biomedical Sciences and Pharmacy, University of Newcastle, \\ 2308, Australia \\ 2 School of Mathematics and Physical Sciences, University of Newcastle, 2308, Australia \\ 3 School of Health Sciences, University of Newcastle, 2308, Australia.
}

Dietary phytosterols are well-known hypocholesterolaemic agents. Curcumin elicits hypolipidaemic and anti-inflammatory effects in preclinical studies; however, consistent findings in humans are lacking. Concurrent phytosterols and curcumin supplementation may exhibit enhanced hypolipidaemic and anti-inflammatory effects to optimise cardio-protection. The aim of this study was to examine the effects of dietary supplementation with phytosterols with or without curcumin on circulating blood lipids. A double-blinded, randomised, placebo-controlled, $2 \times 2$ factorial intervention was conducted in individuals with hypercholesterolaemia. Participants received either placebo (PL), phytosterols (PS), curcumin (CC), or a combination of phytosterols and curcumin (PSCC) for four weeks. Primary outcomes included fasting total cholesterol (TC), LDL-cholesterol, HDLcholesterol, triglycerides (TG), TC-to-HDL-C ratio (TC:HDL-C), and glucose. Seventy participants completed the study (PL, $n=18 ; P S, n=17 ; C C, n=18 ; P S-C C, n=17$ ). PS and PS-CC supplementation significantly lowered TC, LDL-cholesterol, and TC:HDL-C post-intervention $(p<0.05)$. PS resulted in reductions of $4.8 \%$ and $8.1 \%$ in TC and LDL-cholesterol, respectively. CC exhibited reductions of $2.3 \%$ and $2.6 \%$ in TC and LDL-C, respectively. However, PS-CC resulted in a greater reduction in TC $(11.0 \%)$ and LDL-cholesterol $(14.4 \%)$ than either of the treatments alone. The reduction in the PS-CC treatment was significantly greater compared to those for CC $(p<0.05)$ or PL $(p<0.01)$ alone. Plasma HDL-cholesterol and TG concentrations remained unchanged across all groups. Our findings provide evidence of complementarity between phytosterols and curcumin for their cholesterollowering potential. Implications of these findings include the development of functional foods containing both the active ingredients for enhanced lipid-lowering and compliance in hypercholesterolaemic individuals. 


\subsection{Habitual Dietary Nitrate Intake and Cardiovascular Events: A Longitudinal Study}

Zahra Bahadoran ${ }^{1}$, Mattias Carlström ${ }^{2}$, Asghar Ghasemi ${ }^{1}$, Parvin Mirmiran ${ }^{1}$, M. D. Fereidoun Azizi ${ }^{1}$ and M. D. Farzad Hadaegh ${ }^{1}$

1 Research Institute for Endocrine Sciences, Shahid Beheshti University of Medical Sciences, Tehran, Iran

2 Department of Physiology and Pharmacology, Karolinska Institutet, Stockholm, Sweden

Objective: Considering the lack of data on the association between habitual dietary intakes of nitrate $\left(\mathrm{NO}_{3}\right)$ and nitrite $\left(\mathrm{NO}_{2}\right)$ and cardiovascular events, we assessed possible effects of dietary $\mathrm{NO}_{3}$ and $\mathrm{NO}_{2}$, in the context of total antioxidant capacity (TAC) of the diet, with the risk of cardiovascular (CVD) outcomes. Methods: Adult men and women without CVD $(n=2369)$ were recruited from the Tehran Lipid and Glucose Study and were followed for a mean of 6.7 years. Dietary $\mathrm{NO}_{3}$ and $\mathrm{NO}_{2}$ intakes as well as dietary TAC and nitric oxide (NO) index were assessed at baseline (2006-2008). Multivariate Cox proportional hazard regression models were used to estimate the risk of CVD across the median of dietary intakes of $\mathrm{NO}_{3} / \mathrm{NO}_{2}$ and dietary TAC and $\mathrm{NO}$ index. Due to a significant interaction between $\mathrm{NO}_{3} / \mathrm{NO}_{2}$ intake and $\mathrm{TAC}$, stratified analyses were conducted for $<$ and $\geq$ median dietary TAC. Results: Daily mean (SD) dietary $\mathrm{NO}_{3}$ and $\mathrm{NO}_{2}$ intakes were 460 (195) and 9.5 (3.9) mg; mean (SD) dietary TAC and NO index were 1406 (740) and 338 (197) mol TE/100 g. In subjects with lower dietary TAC, a higher intake of $\mathrm{NO}_{3}(\geq 430 \mathrm{mg} / \mathrm{d})$ was accompanied with an increased risk of CVD (Hazard Ratio $=3.28,95 \% \mathrm{CI}=1.54-6.99$ ). There were no significant associations between dietary intakes of $\mathrm{NO}_{2}$, TAC of the diet, and NO index with the occurrence of CVD events during the study follow-up. Conclusion: High habitual intake of $\mathrm{NO}_{3}$, in the context of low TAC of the diet, may increase the risk of CVD outcomes.

\subsection{Evaluation of the Acceptability of Commercially Available Food Products with Varying Whole Grain Content}

W. N. A. Choo, A. Y. L. Tang and I. A. Brownlee

Human Nutrition Research Centre, Newcastle University (Singapore), 0501 172A Ang Mo Kio Ave 8, Singapore 567739, Singapore

Consumption of whole grains is low in most countries worldwide. The perceived taste/organoleptic characteristics or visual appearance of whole grains have been suggested to be major barriers that limit whole grain consumption, although it is unsure which factor may be most important to acceptance. This study aimed to compare the taste acceptability of commercially available breads and breakfast cereals that contained varying amounts of whole grains with different visual appearances. Following ethical approval, participants aged 21-29 years were recruited to take part in blinded, crossover trials of existing ready-to-eat cereals $(n=100$ evaluating products A-C containing $52.9 \%, 24 \%$, and $0 \%$ whole grains, all of which were of similar size, shape (flaked), and light brown colour) and breads $(\mathrm{n}=101$ evaluating products $\mathrm{D}-\mathrm{F}$ containing $100 \%, 50 \%$, and $0 \%$ whole grains, with all products produced by the same manufacturer and brown, off-white, and white in appearance, respectively). Bread colour was masked by presentation under a blue light. Participants carried out a sensory evaluation of all products in their category (using nine-point hedonic scales). Product A (the cereal with the highest whole grain content) had the highest appearance rating $(p<0.001)$ of the three products (mean \pm SD $6.6 \pm 1.4)$ vs. $B(5.8 \pm 1.5)$ and $C(6.1 \pm$ 1.4). Product $A$ was also the most frequently preferred $(p=0.093)$ product $(n=44)$ vs. $B(n=24)$ and $C(n=31)$. Similar numbers $(p=0.957)$ of participants preferred the different breads $(n=32, n=36$, and $n=33$ for products $\mathrm{A}-\mathrm{C}$ respectively). These findings suggest that the effective formulation of whole grain products can overcome negative sensory or visual aspects of the product.

\subsection{Garcinia Humilis (Achacha) Rind Powder Improved Systolic Blood Pressure in Rats with Diet-Induced Metabolic Syndrome}

O. John, S. Wanyonyi, S. Panchal and L. Brown 
Functional Foods Research Group, Institute for Agriculture and the Environment, University of Southern Queensland, Toowoomba 4350, Australia

Garcinia humilis, known as achacha, is a fruit native to Bolivia and Brazil now cultivated in north Queensland. The traditional uses in Bolivia are to suppress hunger and to extract honey with reputed healing properties. Phytochemical analyses of the freeze-dried rind showed procyanidins $(60 \mathrm{mg} / \mathrm{g})$ together with flavonols. This study investigated Garcinia humilis rind as a potential treatment to reverse the cardiovascular, liver, and metabolic changes in diet-induced obese hypertensive rats. Male Wistar rats (8-9 weeks old) were fed a diet high in both simple sugars, including fructose and sucrose, and saturated and trans-fatty acids for 16 weeks, leading to hypertension, dyslipidaemia, central obesity, impaired glucose tolerance, and non-alcoholic fatty liver disease. Intervention with $1.5 \%$ of Garcinia humilis rind powder for the last eight weeks of the protocol decreased systolic blood pressure and left ventricular diastolic stiffness in these rats. Histological analysis showed decreased infiltration of inflammatory cells and collagen deposition in the left ventricle of the heart. In contrast, no changes were observed in the deposition of fat droplets in the liver, body weight, or blood lipid profile. Thus, Garcinia humilis rind powder showed cardioprotection with minimal metabolic or liver changes in this rat model of metabolic syndrome.

\subsection{Food Matrix Influences Postprandial Lipemia and Satiety in Human Subjects}

C. B. Dias 1,2, A. Thompson 2, X. Zhu 2, H. Singh ${ }^{2}$ and M. L. Garg 1,2

1 Nutraceuticals Research Program, University of Newcastle, Callaghan, NSW 2308, Australia

2 Riddet Institute, Massey University, Palmerston North, New Zealand

Elevated postprandial plasma triglyceride levels are associated with increased risk of developing cardiovascular disease. Food matrix may play an important role in the rate of digestion and absorption of fats, and the manipulation of food structure and composition provides an opportunity to potentially improve postprandial lipemia and therefore reduce the risk of developing chronic diseases. This study aimed to compare the postprandial lipemia induced by isocaloric foods containing the same nutrients but differing in food structure. Healthy young adults donated blood samples after an overnight fast and 0.5, 1, 2, 3, 4, and $6 \mathrm{~h}$ after consuming one of three test meals. The meals were liquid (beverage), semi-solid (mousse), or solid (biscuit) and had the same nutrient composition. Appetite sensations were also assessed over the 6-h period. Consumption of the solid food resulted in lower postprandial triglyceride response compared to the liquid $(p=0.037)$. Six hours after the consumption of the solid food, plasma triglyceride levels returned to baseline values $(p=$ $0.575)$; while after the liquid $(p=0.047)$ and the semi-solid $(p=0.032)$, triglyceride levels were still higher than baseline values. The subjective measure of hunger was lower after consumption of the semi-solid compared to the solid $(p=0.047)$ and the liquid $(p=0.007)$. After consuming the liquid, participants' estimation of prospective food intake was greater than after the semi-solid $(p=0.013)$ and the solid $(p=0.028)$. In conclusion, the consumption of a solid food caused lower postprandial lipemia compared to a semi-solid or liquid food delivering identical nutrients, while the semi-solid was more efficient in curbing hunger.

\subsection{Effect of Intermittent Energy Restriction on Endothelial Function: A Pilot Study}

M. Headland ${ }^{1,2}$, P. M. Clifton ${ }^{1,2}$ and J. B. Keogh ${ }^{1,2}$

1 School of Pharmacy and Medical Science, University of South Australia, Adelaide 5000, Australia

2 Sansom Institute for Health Research and Alliance for Research in Exercise, Nutrition and Activity (ARENA), University of South Australia, Adelaide 5000, Australia

It is unknown if acute intermittent energy restriction affects endothelial function. The aim was to compare the effects of two consecutive days of habitual eating with two consecutive days of energy restriction on endothelial function in healthy, normal weight adults. In a four-week randomised cross-over trial, participants followed a dietary intervention of five days of habitual intake and two consecutive days of energy restriction (women $2100 \mathrm{~kJ}$ and men $2500 \mathrm{~kJ}$ ) each week. Endothelial 
function, measured via flow-mediated dilatation (FMD) was recorded at baseline, week 3, and week 4. Visits in weeks 3 and 4 followed either two restricted days or two days of habitual eating, dependent on the order of randomisation. Adherence to diets was determined from dietary checklists. Participants $(n=12)$ had a mean age of $35 \pm 12.2$ years and maintained stable weight throughout the four weeks. On average, endothelial function as measured by \%FMD did not change across the four weeks of the trial and was the same following two consecutive energy-restricted days $(M=9.10 ; S E M=0.48)$, to that following two habitual eating days $(M=9.52 ; S E M=0.42)$. Mean absolute change in artery diameter also did not change between the two conditions $(0.32 \mathrm{~mm}$ vs. 0.34 $\mathrm{mm}$ ). This pilot trial showed that acute energy restriction did not have any effect on endothelial function in healthy, normal weight adult subjects; however, the small number of participants limits the generalisation of the results. The study is ongoing.

\subsection{The Effect of Magnesium Supplementation on Endothelial Function}

\section{J. Byrne ${ }^{1,2}$, J. B. Keogh ${ }^{1}$ and P. M. Clifton ${ }^{1}$}

1 School of Pharmacy and Medical Sciences, Alliance for Research in Exercise, Nutrition and Activity (ARENA), Sansom Institute for Health Research, University of South Australia, Adelaide SA 5001, Australia

2 Dublin Institute of Technology and Trinity College, Dublin, Ireland

Endothelial dysfunction precedes the development of atherosclerosis, leading to cardiovascular disease (CVD). Evidence supports an association between low magnesium (Mg) intake and CVD incidence/mortality and between $\mathrm{Mg}$ intake and endothelial function. Flow-mediated dilation (FMD) of the brachial artery is used as a measure of endothelial function. Our aim was to determine whether FMD was altered by Mg supplementation. In a pilot cross-over study, 19 healthy men and women were randomised to take an over-the-counter magnesium supplement equivalent to the National Health and Medical Research Council of Australia RDI of 400-420 mg/day for men and 310-320 $\mathrm{mg} /$ day for women for one week, or to follow their usual diet for one week. Weight, FMD, and blood pressure were taken after each intervention, and 24-h urine samples, blood samples, and a three-day weighed food diary were collected. Baseline serum $\mathrm{Mg}$ was within the normal range for all participants. No significant difference was seen in serum $\mathrm{Mg}$ between the interventions. Urinary $\mathrm{Mg}$ and urinary $\mathrm{Mg}: \mathrm{Cr}$ ratio increased between interventions ( $p=0.03$ and $p=0.005$, respectively) after the exclusion of two individuals with an incomplete 24-h urine collection. No significant differences in FMD or blood pressure were found between the interventions. A negative correlation was seen between age and FMD $(r=-0.496, p=0.031)$. When adjusted for age, saturated fat was significantly negatively associated with FMD $(p=0.045)$, while BMI showed a positive correlation with FMD $(p=$ 0.034). One week of Mg supplementation did not improve FMD in a healthy population.

\subsection{Association between Diet Quality and Cardiometabolic Health in Australian Adults: A Cross-Sectional Study}

K. M. Livingstone and S. A. McNaughton

Institute for Physical Activity and Nutrition (IPAN), School of Exercise and Nutrition Sciences, Deakin University, Geelong, Australia

Evidence suggests that diet quality is positively associated with cardiometabolic health. However, few studies have included a nationally representative population and a large variety of cardiometabolic markers. The aim of the present study was to investigate the relationship between diet quality and markers of cardiometabolic health in Australian adults. Dietary data, using two 24h dietary recalls, were collected from adults in the cross-sectional 2011-2012 National Nutrition and Physical Activity Survey ( $\mathrm{n}=2121 ; 46.4$ (SE 0.48) years). Diet quality was assessed using the 2013 Dietary Guideline Index (DGI). Multivariate linear regression analyses were used to examine associations between diet quality and blood biomarkers, body mass index (BMI), waist circumference (WC), diastolic and systolic blood pressure, and an overall cardiometabolic risk score. Analyses were adjusted for age (continuous), smoking (categorical), physical activity (binary), education 
(categorical), urban or rural location (categorical), energy misreporting (continuous), dieting (categorical) or atypical dietary intake on day of reporting (categorical), and family history of diabetes (binary). Biomarkers and blood pressure outcomes were further adjusted for BMI. Higher DGI was associated with lower glucose (coefficient -0.009 , SE 0.004; $p$-trend $=0.033$ ), BMI (coef -0.017 , SE 0.007; $p$-trend $=0.019)$ and WC (coef -0.014, SE $0.005 ; p$-trend $=0.008)$. No other associations were observed. Better diet quality was associated with only a small number of favorable markers of cardiometabolic health. The limited associations observed between diet quality and biochemical cardiometabolic markers warrants further investigation. Moreover, the addition of cardiovascular disease incidence and all-cause mortality in longitudinal investigations will further our understanding of the role of diet quality.

\subsection{Dietary Supplementation with Phytosterols or Curcumin Modulates Plasma Endocan Levels in Hypercholesterolaemic Individuals}

R. E. Muddle ${ }^{1}$, J. J. A. Ferguson ${ }^{1}$, R. Thota ${ }^{1}$, L. MacDonald-Wicks ${ }^{2}$ and M. L. Garg ${ }^{1}$

1 Nutraceuticals Research Program, School of Biomedical Sciences and Pharmacy, University of Newcastle, 2308, Australia

2 School of Health Sciences, University of Newcastle, 2308, Australia

Cardiovascular disease (CVD) remains the leading cause of mortality worldwide. Endothelial dysfunction is the first step in the pathogenesis of atherosclerosis. Endocan, a proteoglycan secreted by vascular endothelial cells, is a biomarker of endothelial function that has been shown to be adversely influenced in hyperlipidaemias, inflammatory conditions, and CVD. Phytosterols are wellestablished hypocholesterolaemic agents and preclinical studies suggest that curcumin is hypolipidaemic and anti-inflammatory; therefore, both have the potential to improve endothelial function. The aim of this study was to investigate the effects of phytosterols and/or curcumin on plasma endocan levels in hypercholesterolaemic individuals. A four-week, double-blinded, $2 \times 2$ factorial, randomised placebo-controlled trial was conducted with four parallel groups. Participants received either placebo (PL), phytosterols (PS), curcumin (CC), or phytosterols and curcumin (PSCC). Seventy hypercholesterolaemic adults, aged $18-70$ years, were included. The current study was a sub-analysis of 52 participants ( $P L, n=13 ; P S, n=13 ; C C, n=12$; PS-CC, $n=14$ ) for which plasma endocan values were in the detectable range. Significant reductions in plasma endocan levels were evident post-intervention in the PS $(-19.5 \%)$ and CC $(-17.8 \%)$ groups $(p<0.05)$ compared with the PL $(-5.8 \%)$ and the PS-CC $(-7.5 \%)$ groups. Our findings suggest that dietary supplementation with PS or CC improves endothelial function in hypercholesterolaemic individuals; however, further studies with a larger sample size are warranted to determine whether PS and CC have complementary and/or synergistic effects on improving endothelial function.

\subsection{Cardiovascular Disease Risk Factors in Liver Transplant Recipients - How Are They Managed?}

\section{Marsh ${ }^{1}$, K. McDowall ${ }^{2}$, K. M. Dickinson ${ }^{1}$}

1 Discipline of Nutrition and Dietetics, College of Nursing and Health Science, Flinders University, SA, Australia

2 Department of Dietetics and Nutrition, Flinders Medical Centre, SA, Australia

Both the number of liver transplants performed and recipient survival is increasing, as is the prevalence of post-transplant cardiovascular disease risk factors among this patient group. Previous research among dietitians has identified this as a priority for patient management; however, there is little research on long-term evidence-based obesity and cardiovascular disease prevention in this group. This study aimed to investigate liver transplant unit health care providers' practices, opinions, and barriers to post-transplant cardiovascular disease risk management. A 27-item electronic survey with multiple-choice and free-text questions was distributed to liver transplant units in Australia, New Zealand, the United Kingdom, and North America through relevant professional associations. The survey investigated respondent demographics, awareness of cardiovascular risk factors, patient management, follow-up, guideline usage, practice barriers, and practitioners' opinions. Thirty-seven 
complete responses were obtained. The main findings were a lack in evidence-based guideline usage with only $11(29.7 \%)$ respondents following guidelines, a decrease in long-term post-transplant monitoring of weight, and no body composition monitoring. Five $(13.5 \%)$ respondents agreed that their unit's current management is optimal to prevent post-transplant weight gain, and six $(16.2 \%)$ agreed that practices are optimal to prevent post-transplant cardiovascular disease risk factors. A majority of reponsdents (75.7\%) identified time as a barrier to effective monitoring and management. Other barriers were staffing $(73.0 \%)$, funding $(51.4 \%)$, and resources $(48.6 \%)$. In summary, practitioners working with liver transplant recipients are aware of post-transplant cardiovascular disease risk and agree that management is suboptimal due to barriers and that more research to explore the implementation of guidelines and suggested management strategies is needed.

\subsection{The Effect of Polyphenols on Cardiovascular Risk Factors in Haemodialysis: A Systematic Review and Meta-Analysis}

W. Marx 1,2,*, J. Kelly ${ }^{2}$, S. Marshall ${ }^{2}$, S. Nakos ${ }^{1}$, K. Campbell ${ }^{2}$ and C. Itsiopoulos ${ }^{1}$

1 School of Allied Health, La Trobe University, Australia

2 Faculty of Health Sciences and Medicine, Bond University, Australia

* Corresponding Author

End-stage kidney disease (ESKD) is a major health burden worldwide and a strong risk factor for sudden cardiac death. Polyphenol supplementation might reduce cardiovascular risk factors in haemodialysis patients. The aim of this systematic review was to evaluate the current evidence for polyphenol interventions for cardiovascular risk reduction in haemodialysis patients. Using the PRISMA guidelines, the following databases were searched: Cochrane Library, MEDLINE (vis Scopus), Embase, and CINAHL. Treatment effect was quantified by meta-analysis for measures of lipid profile, inflammation, oxidative stress, and blood pressure. Risk of bias was assessed using the Cochrane Collaboration Risk of Bias tool, and the quality of the body of evidence was assessed by the GRADE methodology. Twelve studies were included for review. Polyphenol interventions utilised in the included studies were soy, cocoa, pomegranate, grape, and turmeric. When data were pooled, polyphenol interventions significantly improved diastolic blood pressure (MD $-5.62 \mathrm{mmHg}(95 \% \mathrm{CI}$ $-8.47,-2.78) ; \mathrm{I}^{2}=2 \% ; p=0.0001 ; \mathrm{n}=4$ studies; $\mathrm{n}=245$ participants), triglyceride levels (MD -26.52 $\mathrm{mg} / \mathrm{dL}$ (95\% CI -47.22, -5.83); $\mathrm{I}^{2}=57 \% ; p=0.01 ; \mathrm{n}=4$ studies; $\mathrm{n}=191$ participants), and myeloperoxidase (MD -90.1 (95\% CI -135.8, -44.4); $\mathrm{I}^{2}=0 \% ; p=0.0001 ; \mathrm{n}=2$ studies, $\mathrm{n}=126$ participants). Included studies generally had low or unclear risks of bias. The results of this review provide preliminary support for the use of polyphenol interventions in improving cardiovascular risk markers in haemodialysis patients. Due to the limited number of studies that have investigated individual polyphenol interventions, further studies are required to provide recommendations on which polyphenol intervention and dosage is superior.

\subsection{Professional or Volunteer-Delivered Lifestyle Interventions - Which Obtain Better Outcomes?}

\section{Kent, P. Rankin and D. Morton}

Lifestyle Research Centre, Avondale College of Higher Education, Cooranbong, NSW 2265, Australia

Background: Lifestyle modification programs appear effective in the treatment of chronic disease. The Complete Health Improvement Program (CHIP) has demonstrated significant reductions in selected chronic disease risk factors among large cohorts from several countries, including Australia. This study compared the outcomes of participants in professional versus volunteer-facilitated programs. Methods: Paid facilitators (professional) delivered the CHIP intervention through the Swedish American Center for Complementary Medicine, while unpaid (volunteer) facilitators delivered the intervention through community centres. Changes in body mass index (BMI), blood pressure (systolic (SBP) and diastolic (DBP)), blood lipid profile (total cholesterol (TC), high-density lipoprotein (HDL), triglycerides (TG), low-density lipoprotein (LDL)) and fasting plasma glucose (FPG) were compared in 3158 participants attending professionally delivered 
programs ( $34.3 \%$ men, $65.7 \%$ women; mean age $=54.0 \pm 11.4$ years) and 7115 participants treated via volunteer delivery ( $33.4 \%$ men, $66.6 \%$ women; mean age $=57.4 \pm 13.0$ years), 30 days after intervention commencement. Results: After controlling for age, gender, change in BMI, and the baseline biometrics, participants in professionally delivered programs achieved significantly greater reductions in BMI $(p<0.001)$ and HDL $(p<0.001)$ than volunteer-delivered programs. Conversely, participants attending volunteer-delivered programs showed greater reductions in SBP $(p<0.001)$, DBP $(p<0.001)$, TC $(p=0.004)$, LDL $(p<0.001)$, TG $(p=0.006)$, and FPG $(p<0.001)$ at 30 days. Conclusion: The changes in the various biometrics following the CHIP intervention were differentially related to the facilitator type. The implications of this for practice are discussed. Nevertheless, volunteer-delivered programs do not appear to be any less effective, which is noteworthy as volunteers may provide important social capital in the combat of chronic disease.

\subsection{Bidirectional Association between Non-Alcoholic Fatty Liver Disease and Hypertension: Focusing on New, Resolute, and Persisting Cases}

P. Liu, Y. Tang, L. Xiao, X. Zhu, X. Guo, J. Liu, M. He, J. Yuan, Y. Wang, S. Wei, W. Chen, X. Zhang, X. Miao and P. Yao

Affilliation not provided

Objective: To examine the effect of the change in non-alcoholic fatty liver disease (NAFLD) status on the risk of incident hypertension, and vice versa, during a five-year follow-up. Methods: 6,704 eligible hypertension-free subjects and 9,328 NAFLD-free subjects from a Dongfeng-Tongji (DFTJ) cohort study at baseline were enrolled in the study. A logistic regression model was used to estimate the bidirectional association between NAFLD and hypertension. Results: Among the hypertensionfree subjects, incident NAFLD was associated with incident hypertension, even after adjustment for change in body mass index (BMI) (1.49 (1.26-1.76)). The risk of developing hypertension was robust in persisting NAFLD patients $(1.50(1.27-1.78))$. The turnover of NAFLD was showed a protective effect on incident hypertension $(0.75(0.61-0.92)$ compared to persistent NAFLD patients. Among the NAFLD-free subjects, the risk of new-emerging NAFLD was robust for hypertension status both in no minus yes (1.45 (1.23-1.71)) and yes minus yes (1.61 (1.35-1.92)). Moreover, the hypertension patients with effectively controlled blood pressure (BP) decreased the risk of NAFLD development compared to those without effectively controlled BP $(0.77(0.65-0.93))$. Conclusions: The incidence and persistence of NAFLD are associated with increased risk of hypertension, and vice versa. The management of each disease is beneficial to the other, highlighting the potential influence of both NAFLD and hypertension.

\subsection{The Frequency of Nut Consumption Associated with Decreased Metabolic Syndrome among an Iranian} Population

F. Shirani ${ }^{1}$, N. Mohammadifard ${ }^{2, *}$, F. Sajjadi ${ }^{2}$ and M. Maghroon ${ }^{2}$

1 PhD Candidate, Student Research Committee, Ahvaz Jundishapur University of Medical Sciences, Ahvaz, Iran

2 Isfahan Cardiovascular Research Center, Isfahan Cardiovascular Research Institute, Isfahan University of Medical Sciences, Isfahan, Iran

* Corresponding Author

Background: Metabolic syndrome (MetS) is a clustering of metabolic abnormalities that represents a health problem in both developed and developing countries. Nut consumption could be useful to improve cardiometabolic risk factors and MetS. The associations between nut intake and MetS have yet to be assessed. In this cross-sectional assessment, the associations between the frequency of nut intake and prevalence of metabolic syndrome were examined.

Methods: Stratified cluster random sampling was performed to select 9660 individuals aged $\geq 19$ years based on age, sex, and settlement distribution in three districts of central Iran in 2007. Regular 
intake of walnuts, hazelnuts, almonds, pistachios, and sunflower seeds was assessed using a validated 48-item food-frequency questionnaire and a 24-h recall questionnaire.

Results: Younger and higher educated participants reported significantly higher intakes of nuts and seeds ( $p=0.002$ and $p<0.001$, respectively). After adjusting for potential confounders in the fully adjusted model, more frequent intake of nuts and seeds was not significantly associated with the mean of MetS components. The adjusted odds ratio (OR) (95\% confidence interval) of metabolic syndrome was $0.60(0.45-0.98)$ in participants who consumed nuts $\geq 4$ times/week. Moreover, the risk of MetS occurrence was reduced by $40 \%$ in participants who consumed nuts $\geq 4$ times/week.

Conclusion: We found a statistically significant association between the frequency of nut consumption and prevalence of MetS. Frequent nut and seed consumption, $\geq 4$ times/week, had an inverse association with MetS occurrence. More longitudinal studies are needed to confirm these results.

\subsection{What Is the Evidence for Diet in the Development of Atrial Fibrillation in Adults?}

\section{A. F. Shanahan, M. Miller and K. M. Dickinson}

Nutrition and Dietetics, College of Nursing and Health Science, Flinders University, Adelaide, SA, Australia

Atrial fibrillation $(\mathrm{AF})$ is the most common type of cardiac arrhythmia and increases the risk of stroke, heart failure, and mortality. There are currently no dietary recommendations for AF prevention. Previous studies have been conducted in post-operative patient groups or have investigated sole nutrients. The role of diet in the primary prevention of AF is, therefore, not well established. Previous reports in the literature have proposed mechanisms linking diet and AF, including associations between diet and AF risk factors, namely hypertension, overweight/obesity, diabetes mellitus, and inflammation. A systematic review was conducted to investigate food/nutrient consumption and AF incidence in adults. Electronic databases Medline, Scopus, Web of Science, and Cochrane were searched using defined search terms. Search results were screened according to inclusion/exclusion criteria. Studies published in English, involving human participants aged $\geq 18$ years and without AF at baseline were included. Studies investigating nutrient supplementation were excluded. Overall, 30 prospective cohort studies were included. Results suggested that omega3 polyunsaturated fatty acids and/or fish was not associated with AF incidence. However, alcohol was positively associated with AF, particularly at heavy intakes and among males. The effect of caffeine was mixed, therefore results were inconclusive. Other nutrients/foods, including total fat, total polyunsaturated, monounsaturated, saturated and trans-fat, omega- 6 polyunsaturated fat, magnesium, nuts, chocolate, fibre, and the Mediterranean diet, had poor evidence bases. Due to inconsistency in results and limited research regarding some foods/nutrients, firm conclusions could not be reached. Further research is required, including the consideration of diet compositions/patterns, particularly given the emerging evidence regarding the relationship between overweight/obesity and AF risk.

\subsection{Effects of Omega-3 Polyunsaturated Fatty Acids Combined with Folic Acid and Vitamin B6 Supplementation in Patients with Coronary Heart Disease}

M. Liu ${ }^{1}, \mathrm{H}$. Liang ${ }^{1, *}$ and Z. Wang ${ }^{2}$

1 The Institute of Human Nutrition Medical College of Qingdao University, 38 Dengzhou Road, Qingdao 266021, China

2 Qingdao Fuwai Cardiovascular Disease Hospital, Qingdao 266034, China

* Corresponding Author

This study investigated the effects of omega-3 polyunsaturated fatty acids (n-3 PUFA) combined with folic acid and VB6 supplementation in patients with coronary heart disease (CHD). We conducted a randomized, double-blind, 12-week, placebo-controlled trial involving 201 adults with CHD. The participants were randomly divided into four groups: group A: taking $2 \mathrm{~g} /$ day fish oil (DHA + EPA $600 \mathrm{mg}$ ); group B: taking folic acid $400 \mu \mathrm{g} /$ day + VB610.0 mg/day; group C: taking 2 
g/day fish oil, folic acid $400 \mu \mathrm{g} /$ day + VB610.0 mg/day; and group D: taking a placebo. Venous blood was extracted from study participants before and after the intervention. After the intervention, the patients' low density lipoprotein (LDL), triglycerides (TG), and total cholesterol (TC) levels exhibited a statistically significant decrease, and the high density lipoprotein (HDL) level rose (all $p<0.05$ ). The TG levels of groups A-D, compared with those before treatment, decreased by $0.52 \mathrm{mmol} / \mathrm{L}, 0.35$ $\mathrm{mmol} / \mathrm{L}, 1.08 \mathrm{mmol} / \mathrm{L}$, and $0.08 \mathrm{mmol} / \mathrm{L}$, and the lower levels exhibited statistical significance $(p<$ 0.05). The TC levels of groups $\mathrm{A}-\mathrm{D}$, compared with those before treatment, decreased by 0.71 $\mathrm{mmol} / \mathrm{L}, 0.29 \mathrm{mmol} / \mathrm{L}, 0.26 \mathrm{mmol} / \mathrm{L}$, and $0.02 \mathrm{mmol} / \mathrm{L}$, respectively. The LDL levels of groups A-D, compared with those before treatment, decreased by $0.43 \mathrm{mmol} / \mathrm{L}, 0.88 \mathrm{mmol} / \mathrm{L}, 0.98 \mathrm{mmol} / \mathrm{L}$, and $0.02 \mathrm{mmol} / \mathrm{L}(p<0.01)$. Homocysteine was also reduced in the intervention groups; it decreased in group $C$ fallen by $3.74 \mu \mathrm{mol} / \mathrm{L}$, in group B by $1.95 \mu \mathrm{mol} / \mathrm{L}$, and in group $\mathrm{A}$ by $2.02 \mu \mathrm{mol} / \mathrm{L}(p<0.05)$. The IL-1 $\beta$ levels of groups A-D decreased respectively by $12.06 \mathrm{pg} / \mathrm{mL}, 36.68 \mathrm{pg} / \mathrm{mL}, 37.44 \mathrm{pg} / \mathrm{mL}$, and $18.77 \mathrm{pg} / \mathrm{mL}(p<0.05)$. The n-3 PUFA combined folic acid and VB6 supplementation could improve lipid and inflammatory factor levels in patients with CHD. Also, in the way of improvement of the serum TG, LDL, homocysteine, and IL-1 $\beta$, they presented synergistic effects.

3.20. A Comparative Study of the Effects of Palm Olein, Cocoa Butter, and Olive Oil on Lipid Metabolism in Young Healthy Chinese People

G. J. Sun ${ }^{1, *}$, C. Cheng ${ }^{1}$, D. Wang ${ }^{1}$, H. Xia ${ }^{1}$, F. Wang ${ }^{1}$, X. Yang ${ }^{1}$, T. Q. Pan ${ }^{1}$, S. K. Wang ${ }^{1}$, L. G. Yang ${ }^{1}$, H. X. Lu ${ }^{2}$, G. F. Shu ${ }^{2}$, Y. Q. He ${ }^{2}$, Y. L. Xie ${ }^{3}$ and Y. X. Yang ${ }^{4, *}$

1 Key Laboratory of Environmental Medicine Engineering, Ministry of Education, Department of Nutrition and Food Hygiene, School of Public Health, Southeast University, China

2 Department of Laboratory, Zhongda Hospital Affiliated to Southeast University, China

3 Department of Preventive Care, Zhongda Hospital Affiliated to Southeast University, China

4 National Institute for Nutrition and Health, Chinese Center for Disease Control and Prevention, China

* Corresponding Authors

The prevalence of cardiovascular diseases (CVDs) is grim, and dyslipidemia is an independent risk factor for CVDs. While the "sn-2 hypothesis" conjectures that fatty acids at the sn-2 position of the triglyceride backbone have a major effect on the lipid profile, and there are not enough human trials to verify this hypothesis. Seventy-two subjects were screened and randomly divided into three groups (palm oil, POL; Cocoa Butter, CB; or Olive Oil, OO). After a two-week run-in period, a 16week randomized crossover trial was carried out. It was divided into three phases of a four-week experimental period with a two-week washout period between each phase of the experimental period. The three groups of subjects alternately consumed the Chinese diet enriched with one of three test oils. The physical and biochemical indices of subjects were collected before and after each experimental period. Sixty-seven subjects completed the study, and there were no significant differences in baseline indices among the three groups $(p>0.05)$. After dietary intervention, the levels of triglycerides (TG) and Lipoprotein(a) $(\mathrm{Lp}(\mathrm{a}))$ of the POL-enriched diet were significantly lower than those of the OO-enriched diet $(p<0.05)$; the Lp(a) concentration of the CB-enriched diet was significantly lower than that of the OO-enriched diet $(p<0.05)$. However, there were no significant differences in other indices among the three groups $(p>0.05)$. POL and CB are similar to OO, which has most unsaturated fatty acids located at the sn-2 position on the triglyceride backbone. These oils were found to have almost identical effects on lipid metabolism, so the "sn-2 hypothesis" can be confirmed directly.

\subsection{What Is the Evidence for the Relationship between Diet and Metabolic Risk in Adult Liver or Renal Transplant Recipients? A Review}

X. Tan, K. McDowall and K. Dickinson

Flinders University, Adelaide 5042, Australia 
The number of liver and renal transplant recipients and their prevalence of metabolic risk is increasing. Unlike the non-transplant population, the influence of diet on metabolic risk is controversial and there are no evidence-based dietary guidelines for metabolic risk reduction. Therefore, this review aims to evaluate the evidence for the relationship between diet and metabolic risk in this population. A systematic review of the recent literature was performed from 2006 onwards in five electronic databases and reference lists of all included articles and relevant reviews in the field. Studies assessing diet as an independent predictor of triglycerides, high-density lipoprotein, blood pressure, waist circumference, and body mass index in adult single-organ kidney or liver transplant recipients were eligible for review. Seventeen of 1287 identified unique articles met the inclusion criteria. Nine studies were experimental and eight observational. In three trials each, low fat and cholesterol diets decreased triglycerides and high-density lipoprotein, and sodium restriction decreased blood pressure. In two trials, hypocaloric, low-fat diets reduced obesity. Components of Western-style diets were associated with increased odds of metabolic risk in five studies, whereas components of healthy diets were not significantly associated with metabolic risk in all studies, apart from calcium and abdominal obesity. The evidence is weak for a relationship between diet and metabolic risk in this population. As diet may prevent metabolic risk, more well-designed evidencebased dietary interventions and prospective cohort studies with homogenous indicators and verifiable outcomes are required to investigate this relationship in liver and renal transplant recipients.

\subsection{Association of Energy-Dense Ethnic Food Consumption and Its Potential Impact on the Lipid Profile of} the Three Major Ethnicities in Singapore

\section{S. Dang, S. Suratman, S. K. Gill-Dang and S. A. Suratman}

Faculty of Medical Sciences, Food and Human Nutrition, Newcastle University Singapore, Singapore, Singapore

Hypercholesterolemia is a common risk factor for cardiovascular disease across the three predominant ethnic groups of Chinese, Malay, and Indian in Singapore. Food is considered to be a major component of the cultural heritage of Singapore. In spite of rapid globalisation, local Singaporeans prefer their own ethnic food as it is easily available at food centres and affordable; however, is generally energy-dense due to use of traditional ingredients and cooking methods. Comparatively, healthier food options high in fibre and low in saturated fat and sugar are scarce at food centres. The aim of this exploratory study was to investigate the frequency of ethnic food consumption in Chinese, Malay, and Indian populations in Singapore. Together with nutritional analysis of their food choices and reported physical activity levels, insight can be gained into the impact of their diet on lipid profiles. A total of 74 participants (aged 40 to 65 years) representing each of the abovementioned ethnicities were recruited. A self-administered questionnaire evaluating dietary habits, frequency of ethnic food consumption, physical activity levels, basic knowledge of dietary cholesterol sources, and management was provided. Even though $90 \%$ of all participants were able to correctly identify common dietary sources of cholesterol, nutritional analysis of their ethnic food choices indicated high energy, saturated fat, and sodium intake, and did not meet the recommended dietary guidelines for wholegrains and fibre consumption as encouraged by the Health Promotion Board (Singapore). This study highlights the need to introduce more nutritionally balanced food options for the widely consumed ethnic foods in Singapore.

\subsection{Red Palm Olein Supplementation Increases Antioxidants and Reduces Oxidised LDL Levels in Centrally Overweight Individuals}

Radhika Loganathan ${ }^{1,2}$, Shireene Ratna Vethakkan ${ }^{2}$, Ammu K. Radhakrishnan ${ }^{3}$ and Kim Tiu Teng 1

1 Malaysian Palm Oil Board, Bandar Baru Bangi, 43000 Kajang, Selangor, Malaysia

2 Department of Medicine, Faculty of Medicine, University of Malaya, 50603 Kuala Lumpur, Malaysia

3 Pathology Division, Faculty of Medicine and Health, International Medical University, 126, Jalan Jalil

Perkasa 19, Bukit Jalil, 57000 Kuala Lumpur, Malaysia 
Background: The prevalence of obesity is increasing worldwide. Obesity is a risk factor for many non-communicable diseases, including cardiovascular disease. The present study was designed to investigate the effect of consumption of red palm olein on lipid profile and antioxidant markers in abdominally overweight individuals.

Design: A randomised, single-blind, crossover trial was undertaken to test the chronic effects of diet with red palm olein and palm olein on 53 abdominally overweight subjects. Following screening, subjects underwent a six-week dietary intervention on two occasions in a crossover manner. Fasting blood samples were collected for lipid profiles and antioxidant tests. Results: Significant outcomes were increased in alpha tocopherol $(p<0.0002)$, alpha carotene $(p<0.0001)$, and beta carotene $(p<$ 0.0001) concentrations in the red palm olein group compared to the palm olein group. Additionally, an important observation indicated a critical factor in atherogenesis, namely oxidised low density lipoprotein (LDL), which was found to be significantly reduced $(p<0.0386)$ in the red palm olein group. No difference was observed between both experimental oils in lipid profile (LDL-cholesterol, high density lipoprotein (HDL)-cholesterol, total cholesterol:HDL cholesterol, triglycerides, apolipoprotein B100, apolipoprotein A1, apolipoprotein B100:apolipoprotein A1, and lipoprotein (a)). Conclusion: Red palm olein supplementation significantly increased plasma antioxidant levels, namely alpha carotene, beta carotene, and alpha tocopherol, which are inversely associated with a decrease in oxidised LDL levels. Oxidised LDL is a well-known predictor for oxidative stress and a pro-atherogenic marker. This study could provide a useful platform to introduce red palm olein as a healthy, natural vitamin A-rich oil for people living a sedentary lifestyle and for populations with vitamin A deficiency.

\title{
3.24. Effects of Avena nuda L. on Chinese Patients with Hypertension Associated with Type 2 Diabetes Mellitus
}

\author{
Ruixue Mao, Meihong Xu and Yong, $\mathrm{Li}^{*}$ \\ Department of Nutrition and Food Hygiene, School of Public Health, Peking University, Beijing, China \\ * Corresponding Author
}

As hypertension is reported in over two-thirds of type 2 diabetes mellitus (DM) patients, the prevention and control of these two diseases are in urgent need. In a randomized, single-blinded, 28day centralized trial in China, a total of 73 participants meeting the World Health Organisation (WHO)/ International Society of Hypertension (ISH) hypertension guidelines (1999) diagnostic criteria were selected from 445 patients meeting the American Diabetes Association (ADA) standards of medical care in diabetes (2013) diagnostic criteria between 50 and 60 years of age, and were randomly assigned into one of three groups. The composition of NOG was determined. Subsequently, NOG was daily boiled into porridge as a replacement for the diet group. The rate of adherence to the study diet was $100 \%$ at day 28 . Among the three groups mentioned above, systolic blood pressure (SBP), diastolic blood pressure (DBP), fasting blood glucose (FBG), and postpransial blood glucose (PBG) values declined significantly over the intervention period $(p<0.05)$. The changes in SBP were more favorable for both the $100 \mathrm{~g}$-NOG group and $50 \mathrm{~g}$-NOG group than the diet group $(p<0.05)$. The improvements in FBG were better in the 100 g-NOG group than in the diet group $(p<$ $0.05)$. The $100 \mathrm{~g}-\mathrm{NOG}$ group had the most significant reduction of PBG values $(p<0.05)$. A comparison between the 50 and $100 \mathrm{~g} /$ day NOG replacements to a structured diet and the diet alone suggested that the former intervention provided better benefits to patients with hypertension associated with $\mathrm{T} 2 \mathrm{DM}$ in their disease management and cardiovascular disease risk control.

\subsection{The Impact of Diabetes on Vitamin B Nutritional Levels in Active Pulmonary Tuberculosis Patients}

Qiuzhen Wang, Xiaohong Han, Jiaqi Song, Tingyan Kou and Baoli Du

Institute of Nutrition of Qingdao University, Qingdao, China

(1) Background: Despite the substantial progress in global tuberculosis control, the burden of tuberculosis remains high, especially in developing countries. Also in these areas the prevalence of 
diabetes has increased rapidly in recent years. The association between diabetes and tuberculosis has aroused intensive interests. B-vitamins, the critical coenzyme of many metabolic approaches, play very important roles in energy metabolism. However, the study of water-soluble vitamins in pulmonary tuberculosis-diabetes mellitus (PTB-DM) patients in vivo is rarely reported. Therefore, this study aims to analyze the effect of hyperglycemia on the nutritional status of tuberculosis patients by detecting the content of water-soluble vitamins in urine. (2) Methods: Our study consists of TB hospital-based case-control analyses from November 2015 to August 2016. Forty-five cases (patients with pulmonary tuberculosis and diabetes mellitus, PTB-DM) and 45 controls (patients with simple pulmonary tuberculosis, PTB) were included in this study. Cases and controls were matched by gender and age. We collected their urine samples to assessed the intake of vitamins B1, B2, and niacin. Fluorospectrophotometry was used. (3) Results: The content of vitamin B1 in the PTB-DM group were much lower than that in the PTB group $(89.70 \mu \mathrm{g} / \mathrm{g}$ vs. $118.82 \mu \mathrm{g} / \mathrm{g})$, as were the contents of vitamin B2 and niacin $(11.91 \mu \mathrm{g} / \mathrm{g}$ vs. $18.92 \mu \mathrm{g} / \mathrm{g} ; 0.084 \mathrm{mg} / \mathrm{g}$ vs. $0.293 \mathrm{mg} / \mathrm{g}, p<0.05)$. (4) Conclusion: The nutritional status of B vitamins including vitamins B1, B2, and niacin in PTB-DM patients were significantly lower than those in PTB patients, which may play a role in the impaired energy metabolism in PTB patients with DM.

\subsection{Exploring Staff Knowledge of Diet and Diabetes and the Implications This May Have for Practice}

O. Farrer ${ }^{1}$, M. Miller ${ }^{1}$, A. Yaxley ${ }^{1}$ and K. Walton ${ }^{2}$

1 Flinders University, SA, Australia

2 University of Wollongong, NSW, Australia

The known incidence of diabetes in aged care is twice that found in the general population. The high rate of malnutrition in institutionalized older adults has prompted recommendations for a more liberalized dietary approach to diabetes management in aged care. However, guidelines for implementation are not mandatory and have had variable uptake in practice. Residential aged care staff involved in any aspect of the food service system were invited to participate in a one-off focus group to determine their awareness of changes in dietary recommendations, how this knowledge was obtained, and how this may have impacted on their practice and care for older adults with diabetes. Overall, the focus groups $(n=5)$ were poorly attended ( $n=14$ total participants), with only one organization supporting attendance during work hours. Participants were generally nursing or care staff who had received diabetes education as part of their basic training, although most referred to the experience of a relative with diabetes as the source of their knowledge. Key findings suggest that aged care staff are aware of recommendations for a liberalized diabetic diet; however, most still associate sugar-sweetened foods with poor diabetes control and are conflicted in offering these items to residents with diabetes. As a result, food choices are being restricted by some staff, or serving sizes are made smaller for residents with diabetes. Findings suggest a need for a consistent approach in diabetes management in the form of guidelines and support from aged care providers to adequately support regular staff education.

\subsection{Association between CYP2R1 Polymorphism and 25(OH)D Level in Type 2 Diabetes Mellitus Patients}

Songcheng Yu, Xing Li, Yan Wang, Fei Yu, Zhenxing Mao, Chongjian Wang, Yue Ba and Wenjie Li * College of Public Health, Zhengzhou University, Zhengzhou 450001, China

* Corresponding Author

Background: Type 2 diabetes mellitus (T2DM) is a complex metabolic disease. It has been reported that vitamin D deficiency is linked to T2DM. Also, 25-hydroxylase encoded by CYP2R1 is the very enzyme needed to catalyze hydroxylation at the $C_{25}$ position of vitamin $\mathrm{D}$ to produce 25hydroxy vitamin $\mathrm{D}(25(\mathrm{OH}) \mathrm{D})$, the intermediate metabolite of the active form of vitamin $\mathrm{D}$. Thus, genetic variation of rs10741657, located in the promoter region of CYP2R1, may have an effect on $25(\mathrm{OH}) \mathrm{D}$ level. This study was aimed to investigate the association between rs10741657 and 25(OH)D level. Methods: 210 T2DM patients and 198 controls were included in this case-control study. 
Genotyping work was completed by the TaqMan SNP genotyping platform. The association between rs10741657 and T2DM was analyzed by logistic regression. The difference of 25(OH)D level was investigated by the Wilcoxon rank sum test. Results: The results revealed that no significant association between rs10741657 and T2DM was found in this study (odds ratio (OR) 1.073; 95\% confidence interval (CI) 0.805-1.431; $p=0.632$ ). However, T2DM patients with heterozygous genotype AG had lower 25(OH)D levels compared with the controls $(p=0.020)$. And this was not found for homozygous genotypes (AA $p=0.946$; GG $p=0.723$ ). Furthermore, 25(OH)D levels in T2DM patients with the AG genotype were lower than those with the GG genotype $(p=0.015)$. Conclusion: Genetic variation in the promoter region of CYP2R1 has an effect on $25(\mathrm{OH}) \mathrm{D}$ level in T2DM patients but is not a risk factor for T2DM.

Keywords: type 2 diabetes mellitus; vitamin D; CYP2R1; polymorphism; rs10741657

\subsection{Consuming Honey at Least Twice a Week Reduces Type 2 Diabetes Mellitus Risk: 10-Year Follow-Up of the ATTICA Study (2002-2012) \\ E. Georgousopoulou, M. Hunter, N. D'Cunha, D. Mellor, N. Naumovski, C. Chrysohoou, C. Pitsavos and D. Panagiotakos}

Australian National University, Canberra 0200, Australia

Perceived wisdom is to exclude honey from the diet of people with diabetes due to its sugar content. This has recently been reconsidered due to its low glycaemic index and high levels of bioactives. However, the long-term effect of honey consumption on the risk of developing type 2 diabetes mellitus (T2DM) remains unknown. The present study investigated relationships between frequent honey intake and 10-year T2DM risk. The ATTICA study is a 10-year prospective study of individuals aged 18-89 years living in the Athens metropolitan area, Greece. A random sample of 1514 men (mean age: $44.9 \pm 14.3$ years) and 1528 women (45.6 \pm 13.5 years), recruited in 2001-2002, were followed-up for 10 years (2011-2012). After excluding participants with diabetes at baseline, the working sample consisted of 1485 participants. Among other characteristics, honey consumption was collected via a validated Food Frequency Questionnaire. Diabetes was defined according to American Diabetes Association criteria. The 10-year incidence of T2DM was $12.9 \%$ and did not differ between genders $(p=0.574)$. Regarding honey intake, $23.2 \%$ of participants had at least one teaspoon of honey more than twice a week, regardless of gender $(p=0.342)$. Frequent honey intake was more than threefold higher among T2DM-free participants compared to those who developed T2DM $(24.4 \%$ vs. $7.7 \%$ respectively, $p=0.017$ ). Moreover, frequent honey intake was associated with $70 \%$ decrease in T2DM (Odds Ratio $=0.229,95 \%$ Confidence Interval: 0.061, 0.866), independent of age, gender, body mass index, smoking status, and family history of T2DM. The consumption of honey is therefore not associated with the risk of developing T2DM, and may even have a protective role.

\subsection{Synergistic Effects of Houttuynia Cordata with Metformin on High-Fat Induced Metabolic Disorders}

\section{J. H. Wang and H. Kim}

Department of Rehabilitation Medicine of Korean Medicine, Dongguk University, 814 Siksa-dong, Goyang, Gyeonggi, Korea

Metformin and houttuynia cordata are representative anti-diabetic therapeutics in Western and traditional medicine, respectively. The present study examined the therapeutic effects of houttuynia cordata extract (HCE) and metformin combination in a dysmetabolic mice model. Metabolic disorders were significantly induced by a high-fat diet (HFD) for 14 weeks. A combination of metformin and HCE significantly lowered body weight, abdominal fat, perirenal fat, liver and kidney weight, but did not changed epididymal fat. Metformin + HCE treatment markedly attenuated excessive serum triglyceride (TG), total cholesterol (TC) aspartate aminotransferase (AST), alanine aminotransferase (ALT), and endotoxin, and restored the HDL level. Both HCE and metformin + HCE treatment evidently reduced glucose intolerance and fasting blood glucose via the enhancement 
of AMPK activation. In addition, HCE + metformin combination treatment dramatically suppressed inflammation via the inhibition of proinflammatory cytokines (MCP-1 and IL-6) and lipopolysaccharide (LPS) receptor toll-like receptor 4 (TLR4). Histopathological results showed that metformin + HCE treatment obviously ameliorated fatty liver, intestinal villi shrink, and adipocytes enlargement as compared to the HFD control. Besides, HCE and metformin + HCE treatment markedly altered the abundance of Gram-negative bacteria, especially Escherichia coli and Bacteriodetes fragilis, but not Gram-positive bacteria. Overall, HCE together with metformin cooperatively exerts therapeutic effects via the modulation of gut microbiota, especially the reduction of Gram-negative bacterium resulting in the alleviation of endotoxemia.

\subsection{Fruit Intake in Pregnancy and the Association with Gestational Diabetes Mellitus: A Prospective Chinese Cohort Study}

X. Z. Zhou ${ }^{1}$, R. J. Chen ${ }^{1}$, C. R. Zhong ${ }^{1}$, Y. J. Wu ${ }^{1}$, X. T. Li ${ }^{1}$, Q. Li ${ }^{1}$, W. L. Cui ${ }^{1}$, N. H. Yi ${ }^{2}$, M. Xiao ${ }^{2}$, H. Yin ${ }^{2}$, G. P. Xiong ${ }^{3}$, W. Z. Han ${ }^{3}$, L. P. Hao ${ }^{1}$, X. F. Yang ${ }^{1, *}$ and N. H. Yang ${ }^{1, *}$

1 Department of Nutrition and Food Hygiene, Hubei Key Laboratory of Food Nutrition and Safety, MOE Key Laboratory of Environment and Health, School of Public Health, Tongji Medical College, Huazhong University of Science and Technology, 13 Hangkong Road, Wuhan 430030, China

2 Hubei Maternal and Child Health Hospital, Wuhan 430070, China

3 The Central Hospital of Wuhan, Wuhan 430014, China

* Corresponding Authors

Fruit intake has been shown to be associated with the risk of gestational diabetes mellitus (GDM) in some observational studies; however, the evidence is limited and the findings are inconsistent. We aimed to assess the relation of fruit intake and GDM incidence in Chinese pregnant women. We included 2755 eligible women from the Tongji Maternal and Child Health Cohort. Dietary intakes were assessed using a validated semi-quantitative food frequency questionnaire at 24-28 weeks gestation. GDM was diagnosed based on the results of a 75-g, 2-h oral glucose tolerance test. In the adjusted logistic regression model, odds ratios (ORs) and 95\% confidence intervals (CIs) for GDM were computed for the highest compared with the lowest quintiles of fruit intake. GDM occurred in $248(9.0 \%)$ of 2755 pregnant women. The average fruit consumption was $412.1 \mathrm{~g} / \mathrm{day}$. The adjusted ORs (95\% CIs) for GDM from the lowest to highest quintile of whole fruit consumption were 1.00 (referent), $0.83(0.56,1.25), 0.75(0.49,1.15), 0.56(0.35,0.89)$, and $0.45(0.27,0.75)$, respectively; $p_{\text {trend }}=$ 0.001 . Comparing with the recommended fruit consumption (200 400 g/day) according to the current dietary guidelines for Chinese, our results showed that fruit intake $\leq 200 \mathrm{~g} /$ day was not associated with GDM risk (adjusted OR: 1.17; 95\% CI: 0.81, 1.69), while fruit intake $\geq 400 \mathrm{~g} /$ day was associated with a $32 \%$ lower GDM risk (adjusted OR: 0.68; 95\% CI: 0.49, 0.96). Our findings suggested an inverse association of fruit intake with the risk of GDM in Chinese pregnant women.

\subsection{The Long Effect of Dietary Behavior on the Control of Blood Glucose in Elder Type 2 Diabetics Patients}

Rui Fan, Meihong Xu and Yong Li

Department of Nutrition and Food Hygiene, School of Public Health, Peking University, Beijing, China

Effective glycemic management cannot be achieved without an appropriate dietary behavior, but real dietary behavior during follow-up periods and its long-term outcomes are scarce. Our study aimed to evaluate the long-term effect of dietary behavior and develop a reasonable dietary plan for elderly Chinese T2DM patients. A randomized control trial was carried out. A subgroup of 299 subjects between the ages 50 and 70 years received a 30-day centralized intervention and three-year free-living follow-up. The dietary behaviors were analyzed and blood glucose was measured. After the three-year follow-up, participants with more than $45 \%$ of carbohydrate intake from rice and wheat showed considerable increases in fasting plasma glucose (FPG) (odds ratio (OR) $=0.049,95 \%$ confidence interval (CI) $0.003,0.82)$, 2h-PG (OR = 0.048, 95\% CI 0.003, 0.795), and HbA1c (OR = 0.004, $95 \%$ CI $0.002,0.745)$. Participants with over $20 \%$ of fiber intake from oats showed significant decreases 
in FPG (OR $=19.889,95 \%$ CI 1.179, 35.397), 2h-PG (OR $=38.235,95 \%$ CI 1.171, 54.879), and HbA1c $(\mathrm{OR}=61.067,95 \% \mathrm{CI} 2.468,151.112)$. Obvious declines in FPG, 2h-PG, and HbA1c were observed in those participants with over $15 \%$ of protein intake from plants (OR $=38.31,95 \%$ CI 3.751, 391.293; OR $=28.422,95 \%$ CI 1.286,628.377; OR $=10.731,95 \%$ CI 1.063,107.389). In conclusion, lower intake of rice and wheat, in addition to higher intake of tubers, fungi, oats, and vegetable protein in the long-term had significant effects on controlling glycemic and $\mathrm{HbA1c}$ levels among elderly T2DM patients. Our study will provide strategies for dietary prescription to control glycemic levels.

\subsection{Comparison of the Prevalence of Diabetes Mellitus among Pulmonary Tuberculosis Patients between Eastern and Western China}

J. Cai ${ }^{1}$, A. Ma ${ }^{1, *}$, Q. Wang ${ }^{1}$, X. Han ${ }^{1}$, S. Zhao ${ }^{2}$ and Y. Wang ${ }^{3}$

1 The College of Public Health, Qingdao University, 38 Dengzhou Road, Qingdao, China

2 Linyi Chest Hospital, Linyi, China

3 The Department of Public Health, Lanzhou University, 222 Tianshui South Road, Lanzhou, China

* Corresponding Author

This study aimed to gain a deeper understanding of the differences and their associated factors of the prevalence of diabetes (DM) among pulmonary tuberculosis (PTB) patients between eastern and western areas in China. This cross-sectional study was carried out among 6138 newly-diagnosed PTB patients (aged 45-80 years), who were all registered from September 2010 to March 2013 in local PTB clinics of Shandong (eastern) and Gansu provinces (western) in China. At the same time, 4499 control participants were randomly selected in the same communities. Physical examinations, a structured questionnaire, and 24-h dietary recalls were administered. In the PTB group, the prevalence of DM $(7.7 \%)$ in Shandong was significantly higher than that in Gansu $(5.4 \%, p<0.05)$. In Shandong, PTB patients had 1.40 times (95\% confidence interval (CI) 1.13-1.73) higher odds of having DM compared with the control group; conversely, there was no risk in Gansu. As to living habits, it was showed that current drinking status was positively associated with impaired fasting glucose (IFG) group's odds ratios in Shandong (odds ratio (OR) 1.448, 95\% CI 1.024-2.047); in contrast, there were no variables associated with DM or IFG in Gansu. Compared with PTB patients with DM in Gansu, there were much higher intakes of energy, protein, fat, vitamins A, B2, and E, potassium, sodium, calcium, magnesium, iron, and higher current drinking status in Shandong $(p<0.05)$. In conclusion, the prevalence of DM among PTB patients in the eastern area was higher than that in the western area in China. The intakes of dietary nutrients and the drinking status might be associated with the observed differences.

Acknowledgments: Supported by the National Natural Science fund (No. 81673160).

\subsection{Is White Rice Consumption Associated with an Increased Risk of Type 2 Diabetes? A Systematic Literature Review and Meta-Analysis}

E. Barber, H. Y. C. Lau, Y. T. Y. Lau, Y. N. Nga, L. S. Tai, Z. Zhang, A. Zhu and N. Kellow Monash University, Australia

(1) Background: Evidence from cohort studies investigating the relationship between white rice consumption and the incidence of type 2 diabetes is inconsistent. This review aimed to explore the association between white rice consumption and the risk of type 2 diabetes in Western and Asian/Middle Eastern populations. (2) Method: Seven electronic databases were searched for prospective cohort studies published in English and Chinese up to October 2017, which analysed the association between dietary intake of white rice and the development of type 2 diabetes. Eligible studies were assessed for methodological quality and subjected to qualitative and quantitative synthesis using a random effects model meta-analyses. (3) Results: Eight high-quality cohort studies involving 570,225 participants were included in the review. When the highest and lowest categories of rice intake were compared, no association was found between white rice consumption and the risk of type 2 diabetes in Western (relative risk $(R R)=1.05 ; 95 \%$ confidence interval $(\mathrm{CI}): 0.84,1.31$ ) or 
Asian/Middle Eastern $(1.33 ; 1.00,1.79)$ populations. The pooled relative risk for both populations combined was $1.18(0.98,1.42)$, with significant between-study heterogeneity $(\mathrm{I}=76 \%, p<0.001)$. (4) Conclusion: White rice consumption was not associated with an increased risk of type 2 diabetes in either Western or Asian/Middle Eastern populations. Multiple modifiable and non-modifiable factors contribute to type 2 diabetes risk, and there is insufficient evidence linking the consumption of a single food item such as white rice with type 2 diabetes.

\subsection{Chemical and Physical Changes to Fried Chicken}

F. De Alzaa ${ }^{1}$, C. Guillame ${ }^{2}$ and L. Ravetti ${ }^{3}$

Modern Olives, Lara, Victoria, Australia, 3212 and Boundary Bend Olives, Boundary Bend, Victoria, Australia, 3599 .

Cooking with Extra Virgin Olive Oil (EVOO) may improve the nutritional properties of food. When cooking fatty foods in oil, food absorbs/releases fat and undergoes changes in fatty acid composition. The purpose of this study was to assess changes that occur to chicken when fried in EVOO or canola oil in a commercial kitchen, and to assess the stability of EVOO and canola oil when repeatedly heated to high temperatures. Analysis was undertaken in an ISO17025-accredited laboratory. Chicken was deep-fried in a commercial kitchen in either EVOO or canola oil, and the resultant cooking oil was assessed for polar compounds (degradation by-products), K270 (secondary product of oil oxidation), and bio-phenols at $6,12,18$, and $24 \mathrm{~h}$. The level of antioxidants and the fatty acid profile of the raw and cooked chicken were also assessed. Results showed that EVOO-fried chicken (EVFC) had more than double the antioxidants than canola-fried chicken (CFC), and 18 times more antioxidants than raw chicken. EVFC had $7 \%$ and $20 \%$ more monounsaturated fat than CFC and raw chicken, respectively, and half the amount of trans fats. After $24 \mathrm{~h}$, the remaining EVOO contained 5\% less polar compounds, $160 \%$ more antioxidants (average), and half the K270 of the remaining canola oil. EVOO showed a higher stability under heat than canola oil and produced chicken with a healthier nutritional profile.

\subsection{Fish Oil Supplements: Are Customers Getting What They Pay for?}

K. Tran

Asia Pacific International College, Chippendale NSW 2008

There is strong evidence for the beneficial effects of omega-3 (EPA and DHA) on heart diseases. The American Heart Association and Australia Heart Foundation recommend the weekly consumption of 2-3 servings of fish to achieve 250-500 mg per day of DHA and EPA for the primary prevention of coronary heart disease. As this recommendation is not achievable by many, fish oil supplementation is an easy alternative. However, there has also been controversial evidence about the actual effects of fish oil supplements on health. This paper presents a critical review on fish oil supplements in the past 10 years. It shows that a majority of commercially available fish oil supplements (in Australia, New Zealand, U.S., Canada, Norway, Poland, South Africa) contained lower concentrations of omega-3 than claimed on the labels. More importantly, the majority of fish oils exceeded the recommended indices of oxidative markers. In other words, customers are actually paying for rancid, oxidised oils that may not even be omega-3. Besides advertising health benefits that are not there, this could also represent negative health effects. Research has been focusing on methods such as encapsulation to improve the oxidative stability of omega-3.

\subsection{Currency of School Food Anaphylaxis Guidelines and Management Strategies}

\section{G. Fiocco ${ }^{1}$, L. T. Williams ${ }^{1,2}$ and T. Lawlis ${ }^{1}$}

1 Discipline of Nutrition and Dietetics, University of Canberra, Canberra, ACT

2 School of Allied Health Sciences, Griffith University, Griffith., QLD 
The incidence of food induced anaphylaxis amongst Australian children is increasing, where one in 10 infants are diagnosed with food allergies. The vulnerability of school children, including decreased communication skills to verbalise signs and symptoms of an anaphylactic episode, increases the threatening nature of anaphylaxis. Children at risk of anaphylaxis have a limited quality of life, including restrictions of food intake and social events. Anaphylaxis is preventable with proper management and education. This research assessed the Australian States and Territories Department of Education school food anaphylaxis guidelines using a unique audit tool. A qualitative study was conducted, integrating the Australasian Society of Clinical Immunology and Allergy (ASCIA) 2015 anaphylaxis principles and current guidelines practiced by the Australian Department of Education and Literature searched between 2015 and 2017. The Australian Department of Education, including six states and two territories, put forth anaphylaxis guidelines that were audited against principles and sub-principles present within the created Food Allergy and Anaphylaxis School Management Tool. The results indicated incohesive coordination between ASCIA and the Department of Education. Evidently, the department's anaphylaxis guidelines met all ASCIA principles. Due to the serious nature of anaphylaxis, improved communication, management skills, and education should be implemented within Australian school communities. Cohesion of the Australian Department of Education through the application of ASCIA principles is required to ensure that schools implement safe practices adhering to their duty of care for students.

\subsection{Nut Consumption among Malaysian Adults: Are They Meeting the Malaysian Dietary Guidelines Recommendations?}

\section{Mohamad and Y. M. Tan}

\section{Affiliation not provided}

Nuts represent the nutrient dense plant-based foods that make beneficial contributions to a healthy diet. It is well established that nuts can give protection against coronary heart disease, hypertension, hypercholesterolemia, and gallstone disease. Substantial information on nut consumption among Malaysian adults meeting the recommendations of the Malaysian Dietary Guidelines (MDG) is lacking. Therefore, this study was carried out to determine the extent to which Malaysian adults meet these recommendations. A cross-sectional study was conducted on 92 adults aged 19 to 59 years old in Klang Valley. A structured interviewer-administered questionnaire was used to collect sociodemographic data. Two days of 24-h dietary recall (24-HDR) and a nut consumption questionnaire were used to access dietary and nut intakes. Results showed that among all types of nuts consumed, peanuts were consumed the most $(71.7 \%)$, with men being more frequent consumers $(76.9 \%)$ compared to women (67.9\%). Younger adults (aged 19-29 years) consumed more cashews, hazelnuts, pecans, and macadamias, whilst older adults (aged 30-59 years) preferred peanuts, almonds, pistachios, and walnuts. The mean consumption of various type of nuts and nut products ranged between 0 to $2.09 \mathrm{~g} /$ day, with the average consumption just $7 \mathrm{~g} /$ day, about $46.7 \%$ of the minimum recommendation. Among those who consumed nuts during the two days of 24-HDR showed that nuts actually can contribute to a high percentage (36.1\%) of daily energy intake. We conclude that Malaysian adults failed to meet the MDG recommendations for nut consumption. Action must be taken by authorities to increase nut intake among Malaysian adults.

\subsection{Changing Our Minds - Does Eating a Dish Change a Restaurant Diner's View of Healthiness? \\ C. Cairncross, L. Neill, A. Brown and E. Rush}

Auckland University of Technology (AUT) Food Network, AUT, Auckland, New Zealand

Healthier restaurant options may have a role to play in addressing rising obesity rates worldwide. With the number of restaurants increasing, in addition to the frequency of dining out, many diners are seeking healthier meal options rather than indulgence. The factors that impact diners' perception of healthier choices are still to be elucidated. However, our aim was to investigate the differences between menu perceptions of dish healthiness, perceptions post-consumption, and 
nutrition profile. In November 2015, we invited a group of 34 of Auckland's top chefs, professional chef/restaurant association members, and school nutrition advisors to a five-course dinner at AUT's Four Seasons Restaurant. Nutrient profile scores (NPS) were calculated for each course. Using a traffic light system of green (healthy), orange (unhealthy), or red (very unhealthy), diners ranked each course before (menu description) and after consuming food. The NPS ranking of healthiness was subsequently revealed. The largest change occurred for around $45 \%$ of diners who perceived the Amuse (first course) and Dessert (fourth course) dishes to be healthier following consumption. Over $80 \%$ of diners did not change their rating for the Entrée (second course). The Entrée dish also had the highest number of diners who perceived it to be less healthy once eaten (12\%). Diners' perception of food healthiness varied between its menu description, actual consumption, and objective nutrition profile. This suggests that methods of conveying health or nutrition information to diners need to be explored further considering rising obesity rates and diners' desire for healthier menu options.

\subsection{Trends in Added Sugar Consumption by the Australian Population, 1995 to 2011-2012}

\section{S. J. Davison ${ }^{1}$, Y. C. Probst ${ }^{1,2}$, K. E. Charlton ${ }^{1,2}$ and J. C. Y. Louie ${ }^{3}$ \\ 1 School of Medicine, University of Wollongong, Wollongong 2522, Australia \\ 2 Illawarra Health and Medical Research Institute, Wollongong 2522, Australia \\ 3 School of Biological Sciences, University of Hong Kong, Pokfulam, Hong Kong, China}

Background: Added sugar has been increasingly implicated as a factor in chronic disease development. The aim of this research was to compare trends in added sugar (AS) consumption in the Australian population using two nationally representative dietary surveys, conducted in 1995 (n $=13,858)$ and 2011-2012 ( $\mathrm{n}=12,153)$. Methods: A systematic method was applied to 24-h recall data in both surveys to estimate AS consumption and food sources thereof. Results: Population-level intakes of AS in Australia have decreased from 10.7\% of total energy (\%E) in 1995 to $10.1 \% \mathrm{E}$ in 20112012 , falling closer in line with the recommended maximum of $10 \% \mathrm{E}$. Children and adolescents (1018 years of age) consume the highest amounts. In 1995, children and adolescents aged 10-18 years had the highest \%E at $15.2 \% \mathrm{E}$, whilst in 2011-2012 adolescents aged 14-18 years had the highest \%E at $12.9 \% \mathrm{E}$. Soft drinks remain the largest single contributor to AS consumption, despite a reduced contribution of these to total AS intake over time (contributing 23.6\% of total AS intake in 1995 and 18.4\% in 2011-2012). Conclusion: AS intakes in the Australian population have decreased over time and are closely aligned to international recommendations; however, in some age groups, AS intakes exceed recommendations, warranting public health strategies to address this excess consumption. Strategies targeting sugar-sweetened beverages in children and adolescents are likely to have the greatest impact.

\subsection{Learning, Sharing, and Teaching about Nutrition with Social Media}

\section{K. Klassen ${ }^{1}$, M. M. Adamski ${ }^{1}$, L. Brennan ${ }^{2}$, M. Reid ${ }^{2}$, M. Lim ${ }^{3}$, T. A. McCaffrey ${ }^{1}$ and H. Truby ${ }^{1}$ \\ 1 Department of Nutrition, Dietetics and Food, Monash University, Melbourne, Australia \\ 2 School of Psychology, Australian Catholic University, Melbourne 3000, Australia}

Globally, social media use has reached 2.8 billion users; however, there has been little scientific exploration into how people are using it to communicate about nutrition. The aim of this study was to explore how people use social media to learn and communicate about nutrition. An exploratory, inductive, qualitative approach was employed using comments from a three-week online course "Food as Medicine". Learners (people enrolled) posted comments on a forum in response to course content. Forum posts that mentioned "social media" or related terms were analysed for themes. A total of 26,120 active learners participated in "Food as Medicine" in May 2016. Of these, 9093 learners commented between 1 and 1312 times (median 13) in the forums. Two major themes that emerged were: (1) teaching - where learners advocated for their beliefs and reference to external social media was used to support this advocacy, often citing perceived experts or trusted experts/sources of information. Statements were often preceded by "I think" or "I believe", but could also be presented 
factually; “The advice to reduce saturated fat consumption directly lead [sic] to the advice to increase carbohydrates"; and (2) sharing-where learners shared health information they found "worth watching" or "interesting" on social media. These analyses suggest that learners in an online nutrition course are using social media to learn (seeking information on social media), teach (advocating about what they have learned and/or reinforcing their beliefs), and share (with other learners and with their friends/followers on other platforms) about nutrition.

\subsection{Do Clean Eating Blogs Serve as a Source of Nutritionally Balanced Recipes? A Comparison of Foods with and without Clean Eating Claims}

K. M. Dickinson ${ }^{1}$, M. Watson ${ }^{1}$ and I. Prichard ${ }^{2}$

1 Discipline of Nutrition and Dietetics, College of Nursing and Health Sciences, Flinders University, Bedford Park, SA 5042, Australia

2 Discipline of Health and Exercise Sciences, College of Nursing and Health Sciences, Flinders University, Bedford Park, SA 5042, Australia

In recent years, millions of people have turned to the Internet as a rapidly accessible source of credible health information, including information about diet. People are also turning away from tertiary qualified nutrition professionals and are following the advice of everyday consumers on social media for information related to diet and health. Websites and blogs devoted to food and nutrition have become increasingly popular, especially blogs about clean eating. Clean eating can be defined as eating healthy, whole, unprocessed foods. There is a perception among adult women that foods labelled 'clean' are healthier than regular foods; however, currently it is not known whether the nutrient composition of clean eating recipes are consistent with recommendations for good health. The aim of this study was to compare the nutrient content of comparable foods with and without clean eating claims. Using previously established methods, we systematically searched for foods with clean eating claims and control recipes from eligible websites $(n=172)$. Recipes were analysed for nutrient content using Foodworks 8. Overall, there was no significant difference in energy content, total fat, saturated fat, carbohydrate, and sugar content between foods with and without clean eating claims $(p>0.05)$. Compared with foods without claims, clean eating recipes were significantly higher in sodium, fibre, and protein $(p<0.05)$. In summary, clean eating recipes contain just as much fat, sugar, and energy as many unhealthy processed foods. Despite claiming to be a healthier alternative to processed foods, foods labelled as clean may be misleading and may undermine consumer efforts to eat a healthy diet.

\subsection{The Effect of Astragalus Polysaccharides on the Osteogenic Differenciation of MC-3T3-E1 Cells in the Three-Dimensional Culture System}

Tianlin Gao, Huaqi Zhang and Aiguo Ma *

School of Public Health, Qingdao University, Qingdao 266021, China

* Corresponding Author

Astragalus polysaccharide (AP), as the main ingredient of astragalus, has been applied to bone healing in traditional Chinese medicine. In vitro experiments represent a convenient method to study its mechanism. However, two-dimensional (2D) cell culture can not mimic the cellular state very well in bone tissue. In this study, a three-dimensional (3D) cell culture system was established by encapsuling MC-3T3-E1 osteoblast-like cells into alginate microcarriers. Then APs with different concentrations were added to the 3D cell culture system to determine their effects on the osteoblasts. The cell proliferation and differentiation in the 3D system were determined via CCK- 8 test, alkaline phosphatase (ALP) activity, quantitative real-time polymerase chain reaction (RT-PCR) analysis, and confocal laser immunofluorescence observations. After a 1-day culture, AP had not performed the promotion of cell proliferation. However, the promotion of cell proliferation was observed in AP groups after 3 days of culture. Meanwhile, AP performed the effect of promoting the osteogenic differentiation of MC-3T3-E1 cells, indicated by ALP activity and osteogenic gene expression of Runx2, osteopontin (OPN), and osteocalcin (OCN). It was concluded that AP possessed the effects of 
promoting MC-3T3-E1 osteoblasts proliferation and osteogenic differentiation in the 3D system. Also in this 3D system, the distribution and the cellular state were closer to the condition in the tissue. Therefore, we speculate that the 3D culture system will be of great value for the biological evaluation of nutrients as an in vitro cell replacement method.

3.43. Is There a Difference in the Risk of Disordered Eating Behaviours and Eating Disorders between Nutrition and Dietetic Students and Non-Nutrition Students? A Systematic Review of the Literature

Z. Mohamed Halim ${ }^{1}$, K. Dickinson ${ }^{1}$ and I. Prichard ${ }^{2}$

1 Nutrition and Dietetics, College of Nursing and Health Science, Flinders University, Bedford Park, SA 5042, Australia

2 Health and Exercise Sciences, College of Nursing and Health Science, Flinders University, Bedford Park, SA 5042, Australia

Behaviours such as chronic dieting, binge eating, and self-induced vomiting reflect disturbed eating pathology and place people at risk of developing eating disorders. Nutrition and dietetic (ND) students are highly exposed to information about diet and health, and it has been suggested that this population may be particularly susceptible to these behaviours, although no systematic review has investigated these associations. The purpose of this review was to investigate whether ND students are at an increased risk of disordered eating behaviours and eating disorders as compared to nonnutrition students. Seven databases (Medline, PsycINFO, CINAHL, Scopus, Web of Science, Cochrane Database of Systematic Reviews, and Cochrane Central Register of Controlled Trials) were searched for English-language, peer-reviewed articles that compared ND university students with non-nutrition students using at least one measure of disordered eating behaviour. Twenty-one crosssectional studies were reviewed. No significant differences were found in the risk of disordered eating behaviours between ND students and non-nutrition students when measured using the Eating Attitudes Test. However, ND students exhibited greater cognitive restraint, using both rigid and flexible control of eating behaviour, than non-nutrition students when assessed by the Three-Factor Eating Questionnaire. Other measures of disordered eating behaviour were not widely used among studies and results were inconclusive. Current evidence suggests that ND students have a greater tendency to consciously restrict food for weight control than non-nutrition students, but they do not report greater disordered eating symptomatology.

\subsection{The Relationship between Food Label Use and Dietary Intakes: A Systematic Review}

\section{K. M. Anastasiou, M. D. Miller and K. M. Dickinson}

Nutrition and Dietetics, College of Nursing and Health Sciences, Flinders University, Bedford Park, SA 5042, Australia

Poor diet, resulting from high intakes of packaged foods, is increasingly recognised as a key contributing factor to ill health. Food labels provide information on the nutritional content of packaged foods to consumers, which may influence the consumption of nutrients such as energy, fat, saturated fat, trans-fats, sodium, and added sugars. This review aims to summarise the current evidence for the association between the use of food labels and dietary intakes. The systematic search was conducted in August 2017 using six databases, with 6325 results. Twenty-six studies were included, including cross-sectional studies $(n=20)$, a cohort study $(n=1)$, and randomised controlled trials $(n=5)$. Studies were assessed using the American Dietetic Association Quality Criteria Checklist. Results were inconsistent in reporting a relationship between diet and food label use but indicated that food label use is likely associated with a small but significant effect on dietary intakes. Nutrition facts label use is associated with healthier diets, measured by food frequency questionnaires and 24-h recalls. However, there is insufficient research on the association between dietary consumption and the use of ingredients lists, serving size information, and front-of-pack labels. Using health-related claims may be associated with poor diets; however, evidence is inconclusive. Apart from health-related claims, using food labels is likely associated with healthier 
diets and should continue to be promoted through policies and education programs. Further research in this area is warranted to provide additional information on the impact of ingredients lists, serving sizes, front-of-pack labels, and health-related claims on dietary intakes.

\title{
3.45. Feasibility of a Commercial Smartphone Application for Dietary Assessments in Epidemiological Research
}

\author{
G. L. Ambrosini ${ }^{1}$, M. Hurworth ${ }^{1}$, R. Giglia ${ }^{2}$, G. Trapp ${ }^{1,2}$ and P. Strauss ${ }^{2}$
}

1 School of Population and Global Health, University of Western Australia, Crawley, WA 6009, Australia

2 Telethon Kids Institute, University of Western Australia, Crawley, WA 6009, Australia

The use of mobile phone technology in research may offer multiple advantages over traditional data collection methods. This study tested the feasibility of a commercial smart phone application (app) as a dietary assessment tool in epidemiological research and compared it with a traditional method. Study participants completed a four-day food diary using a modified version of the Easy Diet Diary app and two 24-h dietary recalls during the same week. At the end of data collection, participants completed a questionnaire on their experience with both methods. Average proportions of energy from macronutrients and fibre, iron, and calcium densities from the app and 24-h recalls were compared, using mean agreement, limits of agreement, and Pearson's correlations. The prevalence of dietary underreporting was compared in each method using the Goldberg method. A total of 50 adults ( $82 \%$ women) provided data for analysis (mean age, 31 years; mean body mass index (BMI), $22.4 \mathrm{~kg} / \mathrm{m}^{2} ; 20 \%$ overweight/obese). Participant feedback showed high levels of acceptance for the app, e.g., $83 \%$ preferred using the app to completing 24 -h recalls. The average difference in energy intake (mean agreement) between methods was $268 \mathrm{~kJ} / \mathrm{d}$. For all intakes except alcohol, the average difference between methods was not significantly different from zero. The majority of nutrients showed acceptable limits of agreement and there was little evidence of bias. The prevalence of dietary misreporting was similar in both methods. These findings demonstrate good feasibility for applying this commercially-developed smartphone app in epidemiological research.

\subsection{The 'Typical Aussie Bloke Study': Breakfast Consumption Habits of Young Australian Men}

A. Quatela ${ }^{1}$, A. J. Patterson 1,3,4, R. Callister 2,3,4 and L. K. MacDonald-Wicks 1,3,4

1 Discipline of Nutrition and Dietetics, School of Health Sciences, The University of Newcastle; Callaghan, NSW 2308, Australia

2 School of Biomedical Sciences and Pharmacy, The University of Newcastle, Callaghan, NSW 2308, Australia

3 Priority Research Centre for Physical Activity and Nutrition; The University of Newcastle, Callaghan, NSW 2308, Australia

4 Hunter Medical Research Institute, New Lambton, NSW 2305, Australia

Breakfast is thought to be important for long-term weight maintenance and the prevention of chronic disease development. However, little is known regarding the foods/beverages that currently constitute a typical Australian breakfast. This study aimed to describe the current breakfast habits of young Australian men (aged 18-44 years). Recruitment was from metropolitan (Newcastle) and regional (Tamworth) NSW, Australia for a multicentre cross-sectional study. Participants completed an online survey investigating breakfast consumption habits and other lifestyle characteristics. The sample included 112 men. The majority of participants $(84 \%)$ were habitual breakfast eaters $(\geq 5$ times/week) and consumed this meal early (84\%; 5:01 to 8:00 am). A typical breakfast for the majority of habitual breakfast eaters habitually consisted of one or more of the following beverages and/or foods ( $\geq 5$ times/week): coffee (40.4\%), breakfast cereal with milk (39.4\%), fruit (28.7\%), toast $(13.8 \%)$, spreads $(11.7 \%)$, and/or yogurt $(12.8 \%)$. This breakfast may be augmented (1-4 times/week) to include: eggs $(58.5 \%)$, bacon $(30.9 \%)$, juice $(19.1 \%)$, and/or tea $(17.0 \%)$. It appears that the most common foods consumed by Australian men have not changed since the National Nutrition Survey (NNS) in 1995. Cereals, milk, and fruit were the most common foods consumed in both studies. However, the proportions of foods consumed appear to have changed since the NNS in 1995; for 
example, fruit consumption appears to have increased and milk consumption decreased, suggesting that Australian male breakfast habits may have changed over time.

\subsection{Effect of Lactobacillus casei Supplementation on Liver Injury Induced by Rifampicin and Isoniazid in Rats}

J. Cai, A. Ma*, Y. Wang, Y. Li, H. Hao, J. Zhang and Y. Sun

The College of Public Health, Qingdao University, 38 Dengzhou Road, Qingdao, China

* Corresponding Author

Isoniazid (INH) and rifampicin (RFP) are widely used in anti-tuberculosis therapy recommended by World Health Organisation (WHO), which may induce severe liver injury. This study aimed to detect the protective effect of Lactobacillus casei strain Shirota (LcS) on liver injury induced by RFP and INH in rats. Seventy-two Male SD rats were randomly divided into six groups including a blank control group (CG), a model group (MG), and low-dose, mid-dose, and high-dose LcS groups (LLG, MLG, and HLG), as well as bicyclol group $(B G, n=12)$. All groups except CG (20 $\mathrm{mL} /(\mathrm{kg} \cdot \mathrm{bw} \cdot \mathrm{d})$ physiological saline) were given $50 \mathrm{mg} /(\mathrm{kg} \cdot \mathrm{bw} \cdot \mathrm{d}) \mathrm{INH}$ and RFP by gavage, and after 2 $\mathrm{h}$, LLG, MLG, and HLG were given $10 \times 10^{8}$ colony forming units $(\mathrm{CFU}) /(\mathrm{kg} \cdot \mathrm{bw} \cdot \mathrm{d}), 20 \times 10^{8}$ $\mathrm{CFU} /(\mathrm{kg} \cdot \mathrm{bw} \cdot \mathrm{d})$, and $40 \times 10^{8} \mathrm{CFU} /(\mathrm{kg} \cdot \mathrm{bw} \cdot \mathrm{d})$, while LcS and BG were given $7.5 \mathrm{mg} /(\mathrm{kg} \cdot \mathrm{bw})$ bicyclol by gavage. After 28 days, hepatic function indices, superoxidedismutase (SOD), and malondialdehyde (MDA) were tested by serum. Compared with MG, levels of alanine aminotransferase and alkaline phosphatase in the HLG group decreased by $16.3 \%$ and $14.4 \%$, respectively; the total biliary acid in LLG, MLG, and HLG significantly decreased by $26.0 \%, 39.4 \%$, and $42.1 \%$; the direct bilirubin in MLG and HLG significantly decreased by $22.6 \%$ and $37.9 \%$, and the indirect bilirubin decreased by $13 \%$ and $15.4 \%(p<0.05)$. The content of MDA decreased and SOD increased significantly in the LcS intervention groups. In conclusion, liver injury was observed in groups treated with RFP and INH drugs, which could be improved by LcS supplementation in rats.

\subsection{Traditional Chinese Vinegar May Reduce the Risk of Renal Stone Disease by Modeling Gut Microbiome: An Investigated Hypothesis}

X. B. Xu, ${ }^{1}$, D. Luo ${ }^{2}$ and D. H. Zhang ${ }^{1}$

1 Department of Urology, Zhejiang Provincial People's Hospital, Hangzhou, China

2 Department of Pharmacy, Zhejiang Provincial People's Hospital, Hangzhou, China

The pathophysiology of renal stone formation is unclear, while the calcium oxalate calculus is the most common type, making oxalate an important pathogen. We hypothesize that traditional Chinese vinegar may reduce the risk of renal stone by remodeling the gut microbiome, based on the fact that Shanxi province in North China is famous for producing and consuming great amounts of traditional Chinese vinegar and recent studies have revealed the prevalence of urolithiasis there is unusually low $(0.14 \%)$. Furthermore, we found a negative correlation between vinegar sale volume and the prevalence of urolithiasis in the top five vinegar-producing provinces in China. Studies suggested that the gut microbiome, especially oxalobacter formigenes, may reduce the blood oxalate concentration. Moreover, patients with urolithiasis exhibited an altered gut microbiome. Vinegar has been sugguested to affect health in many ways, yet no connection with urolithiasis has been proposed. We hypothesize that traditional fermented vinegar might help the gut microbiome in its ability to degrade and excrete intestinal oxalate. Through a literature review, we found that during and after fermentation, traditional Chinese vinegar was bioactive in bacteric and metabolic ways, both involving oxalic acid. Further studies are needed to explore the mechanisms of vinegar. For patients with recurring stones or identified changes in the gut microbiome, it may be worth adding more vinegar to the diet. 
3.49. Vitamin B12 and the Presence of Probiotic Strain Bifidobacterium animalis subsp. lactis hn019 (dr10 tm) among Pregnant Women

S. Bardosono, N. Wibowo, L. B. Sutanto, R. Irwinda, R. Cannan, A. Rowan and J. Dekker

Department of Nutrition, Faculty of Medicine, Universitas Indonesia, Jakarta 10430, Indonesia

Introduction: Vitamin B12 plays a role during pregnancy in maintaining folate metabolism. Together with folate and vitamin B6, B12 is involved as a coenzyme in DNA synthesis and various methylation reactions in developing embryos. This study aims to compare vitamin B12 blood levels with respect to the presence of probiotic strain Bifidobacterium animalis subsp. lactis HN019 (DR10 ${ }^{\mathrm{TM}}$ ) among pregnant women in Indonesia. Methods: This study is part of a larger study entitled "Effects of Bifidobacterium animalis subsp. lactis HN019 (DR10TM), inulin, and micronutrient-fortified milk on fecal DR10 ${ }^{\mathrm{TM}}$, immune markers, and maternal micronutrients among Indonesian pregnant women". Further analyses were performed using independent $t$-test or Mann-Whitney test and generalized linear model (GLM)-repeated measures to compare vitamin B12 intake and blood concentration during pregnancy with the presence $(n=22)$ or absence $(n=55)$ of fecal DR10TM at the third trimester. Results: At the first and second trimesters, there were no differences in vitamin B12 blood levels between the two groups, based on the presence or absence of fecal DR10TM. However, at the third trimester, while having similar vitamin B12 intakes, vitamin B12 blood concentrations were significantly higher in the fecal DR10 ${ }^{\mathrm{TM}}$-positive group $(p=0.035)$. Conclusion: The consumption of pre- and probiotics during the periconceptional period may be a useful strategy for improving maternal vitamin B12 status, therefore providing benefits to the offspring's quality of life.

Keywords: Indonesia; pregnancy; probiotic DR10 ${ }^{\mathrm{TM}}$; vitamin B12

\subsection{Efficacy of Synbiotics among Constipated Adults in Serdang, Selangor}

\section{Y. J. Lim ${ }^{1}$, J. Rosita ${ }^{1}$, A. S. Hazizi ${ }^{1}$ and J. Y. Chieng ${ }^{2}$}

1 Department of Nutrition and Dietetics, Faculty of Medicine and Health Sciences, Universiti Putra Malaysia, 43400 UPM Serdang, Selangor, Malaysia

2 Gastroenterology and Hepatology Unit, Department of Medicine, Faculty of Medicine and Health Sciences, Universiti Putra Malaysia, 43400 UPM Serdang, Selangor, Malaysia

The complementary and synergistic synbiotics approach leads to the balance of gastrointestinal microbiota and improvement in bowel functions. A randomized, double-blinded, placebo-controlled study was conducted to examine the efficacy of a synbiotics supplement among constipated adults. A total of 85 constipated adults, diagnosed by Rome III criteria for functional constipation, were randomized to receive either synbiotics $(n=43)$ or placebo $(n=42)$ once daily $(2.5 \mathrm{~g})$ in the morning for 12 weeks. Follow-up was done every fortnight with treatment response based on a questionnaire that included a record of evacuation (stool frequency, stool type according to Bristol Stool Form Scale), constipation symptoms (stool, rectal, and abdominal symptoms), and quality of life (physical discomfort, psychosocial discomfort, worries and concerns, and satisfaction). Physical discomfort was found to improve significantly in synbiotics than the placebo group (interaction group/time, $p<$ 0.05). There were no significant differences in the stool evacuation, but defecation frequency and stool type in the treatment group were improved tremendously compared to the placebo group. While the treatment group was reported to have higher reduction in the severity of constipation symptoms, the differences were not statistically significant. Dietary supplementation of synbiotics in this study suggested that the combination of probiotics and prebiotics improved evacuation and constipation symptoms as well as quality of life, although not significantly. This was due to the high placebo effect, as synbiotics failed to demonstrate benefit over the controls. 
3.51. High-Sulforaphane Broccoli Sprouts Powder Reduces Serum Nitric Oxide Metabolites in Helicobacter Pylori-Infected Patients

Zahra Bahadoran, Parvin Mirmiran, Asghar Ghasemi and M. D. Fereidoun Azizi

Research Institute for Endocrine Sciences, Shahid Beheshti University of Medical Sciences, Tehran, Iran

Objective: Pathological overproduction of nitric oxide (NO), induced by Helicobacter pylori $(H$. pylori), is known as a risk factor for the development of gastritis and gastric cancer. The current study investigated the effect of high-sulforaphane (SFN) broccoli sprouts powder on serum NO metabolites in $H$. pylori-infected patients. Methods: Eighty-six patients with positive $H$. pylori stool antigen test (HpSAg) were randomized to receive one of the three following regimens: STT-Standard triple therapy for 14 days; BSP $-6 \mathrm{~g}$ /day broccoli sprouts powder for 28 days; and combination of these, denoted as STT (for 14 days) + BSP (for 28 days). Fasting serum NO metabolites were assessed at baseline and again after treatment. Results: The mean age of participants was $50.8 \pm 13.0$ years. The eradication rates of $H$. pylori assessed by HpSAg were $89.3 \%, 56.0 \%$, and $91.7 \%$ in the STT, BSP, and STT + BSP groups, respectively. The median (inter-quartile range) serum NOx level of the participants was $44.8 \pm 22.0 \mathrm{~mol} / \mathrm{L}$, at baseline. There was a significant decrease in serum NOx levels in BSP (25.3 vs. $48.8 \mathrm{~mol} / \mathrm{L}, p<0.001)$ and STT + BSP (32.2 vs. $43.9, \mathrm{~mol} / \mathrm{L}, p<0.05)$ groups after intervention. The median change percentages of serum NO metabolites were $-2.2,-38.2$, and $26.1 \%$ in the STT, BSP, and STT + BSP groups, respectively. Conclusion: We concluded that high-SFN broccoli sprouts, a natural antibacterial and antioxidant agent, besides exhibiting considerable beneficial effects on $H$. pylori infection, may attenuate undesirable $\mathrm{NO}$ overproduction and consequently lead to healthpromoting outcomes in patients.

\subsection{Effects of Goat and Cow Milk Powder-Based Diets on Intestinal Bacteria and Metabolic Activity in Newly Weaned Rats \\ G. Paturi 1, C. A. Butts 2, D. Hedderley 2, H. Stoklosinski 2, S. Martell 2, H. Dinnan ${ }^{2}$ and \\ E. A Carpenter. ${ }^{3}$ \\ 1 The New Zealand Institute for Plant \& Food Research Limited, Auckland, New Zealand \\ 2 The New Zealand Institute for Plant \& Food Research Limited, Palmerston North, New Zealand \\ 3 Dairy Goat Co-operative, 18 Gallagher Drive, Hamilton 3206, New Zealand}

The synergistic prebiotic effects of goat and cow milk powders and the known prebiotics (inulin, oligofructose), as measured by changes in intestinal bacterial populations and organic acid concentrations, were investigated in newly weaned rats. After 21 days of feeding diets containing the milk powers with and without inulin and oligofructose, the rats fed diets containing prebiotics had the lowest food intakes and body weights. Prebiotics added to the diet led to the promotion of intestinal Bifidobacteria. Goat and cow milk powder-based diets influenced the bacterial populations in the gastrointestinal tract (ileum, caecum, or colon). The pathogenic bacterium Clostridium perfringens was decreased significantly in rats that consumed the goat and cow milk powder-based diets. In the large intestine, the concentrations of short-chain fatty acids, acetic, butyric, and propionic, were increased by the prebiotics. Fermentation indices of short chain fatty acids (SCFAs) in both the caecum and colon were lower in rats fed goat milk powder-based diets, indicating that goat milk may possess anti-inflammatory properties. Overall, the goat and cow milk powders alone and combined with prebiotics resulted in different effects on intestinal bacterial composition and metabolism.

3.53. Probiotics Consumption: A Systematic Review and Meta-Analysis of Effects on Serum Concentrations of Liver Function Enzymes

C. Irwin ${ }^{1}$ and $S$. Khalesi ${ }^{2}$

1 Menzies Health Institute Queensland, School of Allied Health Sciences, Griffith University, Gold Coast, Australia 
2 School of Health, Medical and Applied Sciences, Central Queensland University, Rockhampton, Australia

The human gastro-intestinal tract is a densely populated ecosystem of microorganisms, and interactions between the gut and liver are well recognised. Thus, the modulation of gut bacterial flora may improve liver function and reduce or reverse the progression of chronic liver diseases. This study employed systematic review and meta-analytic procedures to clarify the effect of probiotics and synbiotics (probiotics + prebiotics) consumption on serum concentrations of liver enzymes (aspartate aminotransferase (AST), alanine aminotransferase (ALT), alkaline phosphatase (ALP), gamma-glutamyl transpeptidase (GGT), albumin, and bilirubin). A systematic search of the online databases PubMed (MEDLINE), CINAHL, and Cochrane Library through to March 2017 identified a total of 15 studies (16 trials) employing randomised or non-randomised controlled trials where probiotics or synbiotics treatment was compared agaist a control or placebo treatment. Consumption of probiotics and synbiotics significantly reduced serum ALT $(-9.23 \mathrm{IU} / \mathrm{L}, 95 \%$ confidence interval (CI): -15.14 to -3.32$)$; AST (-9.00 IU/L, 95\% CI: -16.31 to -1.70$)$, and GGT ( $-8.40 \mathrm{IU} / \mathrm{L}, 95 \%$ CI: -12.61 to -4.20). No significant differences were observed between the intervention and control groups for changes in the serum concentration of ALP, albumin, and bilirubin $(p>0.05)$. Subgroup analysis suggested the existence of liver disease at baseline, synbiotics, and supplementation duration $\geq 8$ weeks resulted in more pronounced improvement in liver function enzymes. Probiotics and synbiotics consumption may lower serum concentrations of liver enzymes commonly used in clinical practice as biomarkers of liver function. However, the mechanism of the effect is not clear and requires further investigation.

\title{
3.54. Goat Milk Products and Their Impact on an Infant Microbiome
}

\author{
J. A. Mullaney 1,2, L. Samuelsson 1 , C. Thum 1,2, N. C. Roy 1,2,3 and W. Young 1,3 \\ 1 AgResearch Ltd., Grasslands, Palmerston North, New Zealand \\ 2 The High-Value Nutrition National Science Challenge, Auckland, New Zealand \\ 3 Riddet Institute, Massey University, Palmerston North, New Zealand
}

Goat milk is compositionally different from cow milk, and consumers who are unable to tolerate one type of ruminant milk may tolerate another better. These differences are likely to be influenced by bacterial metabolism of milk components.

Faecal fermentation experiments using different goat and cow milk-based substrates (such as whole milk, skim milk, and whey) were undertaken to better understand the difference between goat and cow milk on the composition of the infant large bowel microbiome. We hypothesised that the faecal microbiome composition and function would differ between goat and cow milk substrates. The milk-based components were subjected to an in vitro model of digestion and then used as substrates in anaerobic cultures inoculated with faecal material from a seven-month-old infant, whose diet comprised both breast milk and solids. Effects of the substrates were assessed by subsequent analysis of the microbiomeme, metabolite, and fermentation profiles. The primary fermentation pathway used by the faecal microbiome was likely a mixed acid fermentation, indicated by increased lactate, acetate, succinate, and formate concentrations over time. However, significant increases in butyric acid and propionic acid concentrations over time indicated that other fermentation pathways were also employed. Fermentation profiles showed minor differences between goat and cow milk products. In particular, goat milk whey resulted in higher levels of butyric and propionic acid than cow milk whey. Microbiome and nuclear magnetic resonance metabolomics analyses are currently underway, and will provide clearer insights into how the different milk-based substrates are utilised by the infant faecal microbiome.

\subsection{Banana Peel Extract Induces Serotonin Release but Does Not Affect Stool Consistency in Male Wistar} Rat

W. Fatchurohmah ${ }^{1}$, A. Meliala ${ }^{2}$ and R. C. Sulistyaningsih ${ }^{3}$

1 Departement of Physiology, Faculty of Medicine, Jenderal Soedirman University, Indonesia

2 Departement of Physiology, Faculty of Medicine, Gadjah Mada University, Indonesia 
3 Departement of Pathology Anatomy, Faculty of Medicine, Gadjah Mada University, Indonesia

Gastrointestinal motility is correlated with transit time and stool consistency. Serotonin is an important neurotransmitter, which has role in intestinal motility. Most serotonin is produced in the enterochromaffin cells (EC). Banana peel is a potential natural source of serotonin precursor, as it contains tryptophan, 5-hydroxytryptophan (5-HTP), vitamins, and minerals. The effect of banana peel extract on serotonin immunoreactivity and stool consistency in male Wistar rat is yet unclear. The objective of this research is to investigate the effect of banana peel extract on serotonin immunoreactivity and stool consistency in male Wistar rat. Twenty-five male rats (8-10 weeks old), were divided into five groups: Control Group (KN), Positive Control Group (KP) given 5-HTP 1.50 $\mathrm{mg} / \mathrm{kgBW}$, Banana Peel Extract Group given $4 \mathrm{~g} / \mathrm{kgBW}$ (EP1), Banana Peel Extract Group given 8 $\mathrm{g} / \mathrm{kgBW}$ (EP2), and Banana Peel Extract Group given $16 \mathrm{~g} / \mathrm{kgBW}$ (EP3). Stool consistency score was measured to examine stool consistency. Immunohistochemistry staining was measured to examine serotonin immunoreactivity. Rats in the KP group (235.4 \pm 90.6$)$ showed no significant difference in serotonin immunoreactivity compared with the EP1 group (258.1 \pm 53.6$)$, EP2 group (250.1 \pm 44.8$)$, and EP3 group ( $307.3 \pm 69.2)$. However, rats in the EP3 group showed a significantly higher serotonin immunoreactivity $(p=0.004)$ compared to the control $(199.9 \pm 20.7)$. The stool consistency scores of the EP1 and EP3 groups were higher compared to the control, but not significantly. The administration of banana peel extract at a dose of $16 \mathrm{~g} / \mathrm{kgBW}$ induces significant serotonin release, but was related with stool consistency.

Keywords: serotonin; immunoreactivity; stool consistency; banana peel; 5-HTP

\subsection{Short-Term Dietary Intervention Using the Paleolithic Diet Does Not Impact Serum Trimethylamine- $\mathrm{N}$-Oxide Concentration}

A. Genoni ${ }^{1}$, P. Lyons-Wall ${ }^{1}$, J. Lo ${ }^{2}$, M. Boyce ${ }^{2}$, C. Christophersen ${ }^{1}$ and A. Devine ${ }^{1}$

1 School of Medical and Health Sciences, Edith Cowan University, Perth, WA 6027, Australia

2 School of Science, Edith Cowan University, Perth, WA 6027, Australia

Trimethylamine oxide (TMAO) is produced in vivo by the microbiota of the bowel, from the dietary consumption of choline, phosphatidylcholine, and L-carnitine, predominately found in meat, liver, and eggs. TMAO has previously been associated with cardiovascular disease (CVD), CVD mortality, and metabolic disease. The clinical significance of TMAO levels in healthy populations is yet to be understood. The Paleolithic diet promotes the consumption of foods rich in TMAO precursors. Therefore, we aimed to assess fasting serum TMAO levels in healthy women $(n=35)$, before and after a four-week dietary intervention using both the Paleolithic $(\mathrm{n}=21)$ and Australian Guide to Healthy Eating (AGHE) $(\mathrm{n}=14)$ diets. Pre- and post-intervention serum TMAO concentrations were determined using HPLC-MS/MS. There was a significant increase in servings of protein foods in the Paleolithic group when compared to the AGHE group (1.47 servings/day vs. -0.22 servings/day, $p<0.01$ ), and this was attributed to significant increases in red meat and egg consumption ( 0.65 and 0.22 servings/day, $p<0.05$ ). There were no differences found between groups post-intervention (Paleolithic mean 18.13 $\pm 25.8 \mathrm{M}$, AGHE $15.84 \pm 24.2 \mathrm{M}, p=0.722$ ) or the change in serum TMAO concentration (Paleolithic mean change 6.2 $\pm 33.6 \mathrm{M}$, AGHE 9.2 $\pm 24.7 \mathrm{M}, p=0.78$ ). The use of general linear modelling, with the addition of covariates protein $(\mathrm{g})$, fat $(\mathrm{g})$, red meat and egg intake (servings), showed no effect of dietary treatment group on serum TMAO $(p>0.18)$. Despite increased intakes of protein and eggs, our findings indicate that more research is needed to understand how dietary intake and the gut microbiome interact in altering TMAO levels and the subsequent relationship to cardiometabolic risk.

\subsection{Bifidogenic Effects of Synbiotics in Healthy Infants: Implications for Gut Health}

S. Wang 1, N. Phavichitr ${ }^{2}$, R. Martin 1, S. Intarakhao ${ }^{3}$, R. Tantibhaedhyangkul ${ }^{2}$, J. Knol ${ }^{4,5}$ and S. Jongpiputvanich ${ }^{6}$

1 Nutricia Research, Danone Nutricia Early Life Nutrition, Singapore, Singapore 
2 Department of Pediatrics, Phramongkutklao Hospital, Thailand

3 Department of Pediatrics, Thammasat University, Thailand

4 Nutricia Research, Danone Nutricia Early Life Nutrition, The Netherlands

5 Laboratory of Microbiology, Wageningen University, The Netherlands

6 King Chulalongkorn Memorial Hospital, Department of Pediatrics, Chulalongkorn University, Thailand

Human milk from healthy mothers is considered the optimal nutrition for infants and has been found to be non-sterile in the recent decade. It is estimated to contain about $10^{4}$ culturable bacterial cells/mL [1]. The infant's gut is normally dominated by infant-type Bifidobacteria species such as $B$. breve. Bifidobacteria play key roles in early life gut health and immune maturation. However, not all infants are dominated by Bifidobacteria and some are even devoid of them [2]. Synbiotics can be applied to increase the Bifidobacterial level in the infant's gut. Effects of synbiotics on gut health are dose- and strain-dependent. An exploratory, multicentre randomised, double-blind, placebo-control study was conducted in Thailand to evaluate the bifidogenic effect of two different doses of synbiotics in 290 healthy infants aged from 2-5 months. Infants received either control product (non-hydrolysed cow's milk-based formula) or one of the investigational products (non-hydrolysed cow's milk-based formula supplemented with $0.8 \mathrm{~g} / 100 \mathrm{~mL}$ scGOS/lcFOS with B. breve $\mathrm{M}-16 \mathrm{~V}$ at a dose of either $1 \times 10^{4}$ $\mathrm{cfu} / \mathrm{mL}$ (Syn4) or $1 \times 10^{6} \mathrm{cfu} / \mathrm{mL}$ (Syn6)). Exclusively breast-fed infants were included as a reference. The results showed both Syn4 and Syn6 significantly increased Bifidobacteria proportion and counts in healthy infants when compared with controls. Here, we demonstrate that an infant-type Bifidobacterium, B.breve M-16V, combined with scGOS/lcFOS at a dose close to human milk, can increase Bifidobacteria proportion in healthy infants. This may help infants to create a preferred gut environment and promote gut health during early life.

1 Heikkila, M.P.; Saris, P.E. Inhibition of Staphylococcus aureus by the commensal bacteria of human milk. J. Appl. Microbiol. 2003, 95, 471-478.

2 Tannock, G.W.; Lee, P.S.; Wong, K.H.; Lawley, B. Why Don't All Infants Have Bifidobacteria in Their Stool? Front. Microbiol. 2016, 7, 834.

\title{
3.58. The Microbiome and Gastrointestinal Comfort: Is There a Link?
}

\author{
W. Young ${ }^{1,2,3}$, R. Gearry ${ }^{2,4}$, P. Cotter ${ }^{5,6}$, K. Fraser ${ }^{1,2,3}$ and N. C. Roy ${ }^{1,2,3}$ \\ 1 Food Nutrition \& Health Team, AgResearch Grasslands, Palmerston North, New Zealand \\ 2 The High-Value Nutrition National Science Challenge, Auckland, New Zealand \\ 3 Riddet Institute, Massey University, Palmerston North, New Zealand \\ 4 University of Otago, Christchurch, New Zealand \\ 5 Teagasc, Food Research Centre, Moorepark, Ireland \\ 6 APC Microbiome Institute, University College Cork, Cork, Ireland
}

Irritable Bowel Syndrome (IBS) is a functional gastrointestinal (GI) disorder featuring chronic or recurrent abdominal discomfort, usually with changes in GI habit. However, the mechanisms responsible for IBS are poorly understood. Although alterations in the GI microbiome have been implicated in IBS, there is a lack of consensus on what the exact role of the microbiome is, and how it changes in IBS. To gain a better understanding of the link between the microbiome and IBS, we undertook shotgun metagenomic sequencing of faecal samples from a case-control study. The overall aim of the study was to identify microbial and metabolomic factors that provide mechanistic insights into functional GI disorders and increase the predictability of phenotypes for use in nutrition intervention studies. One hundred and twelve faecal samples from individuals with functional GI symptoms (cases) or asymptomatic individuals (controls) were shotgun sequenced using the Illumina NextSeq platform. Taxonomic classifications were determined using Metaxa2 and the SILVA 128 database. Gene functions were assigned the MG-RAST pipeline. Bacterial genera that differentiated case-controls from IBS groups included Prevotella, Bifidobacterium, Roseburia, and Streptococcus, which were relatively more abundant in those with IBS. Gene functions that best separated groups included those related to carbohydrate metabolism (higher in IBS compared to case-controls), protein metabolism, and virulence factors. Metagenomic sequencing results suggest that carbohydrate fermentation by the microbiome may be an important factor in IBS. Our study 
reveals that the search for consistent microbial markers of IBS should include analyses of microbial function rather than just microbial composition.

\subsection{Protective Effect of Aplysin on Intestinal Floras in Ethanol and Iron-Treated Rats}

Y. $\mathrm{Ma}^{1}$, R. $\mathrm{Li}^{1}{ }^{1}, \mathrm{Y} . \mathrm{Liu}^{2}$ and H. Liang ${ }^{1}$

1 Institute of Human Nutrition, College of Public Health, Qingdao University, Qingdao 266021, China

2 Laboratory of Cellular and Molecular Biology, College of Basic Science, Qingdao University, Qingdao 266071, China

Based on the protective effect of Aplysin in ethanol-treated rats, this study investigated the effect of Aplysin on intestinal flora against ethanol-induced hepatic injury exposed by iron. One hundred and eight healthy male Wistar rats (aged 2 months) were randomly divided into nine groups (12/group). The rats were divided into the following groups: control (normal saline); ethanol-model (50\% ethanol, $8 \mathrm{~mL} \mathrm{~kg}^{-1} \mathrm{day}^{-1}$ for 2 weeks $+12 \mathrm{~mL} \mathrm{~kg}^{-1}$ day $^{-1}$ for 10 weeks); iron-model $(1000 \mathrm{mg} / \mathrm{kg}$ in diet); ethanol+iron; Aplysin (150 mg kg-1 day-1); Aplysin + ethanol; Aplysin + iron; Aplysin + ethanol + iron; positive control (ethanol + iron + glycyrrhizic acid $200 \mathrm{mg} \mathrm{kg}^{-1} \mathrm{day}^{-1}$ ). The study period was 12 weeks. The histopathology of the small intestine was observed with transmission electronic microscopy (TEM); the quantitative real-time PCR (RT-PCR) method was utilized to determine the levels of the gut microbiota. TEM indicated that ethanol or iron treatment resulted in incomplete cell junctions and sparse microvillus, which were even worse in the ethanol + iron group, while Aplysin treatment counteracted such changes. As RT-PCR indicated, compared with the control group, the amount of Escherichia coli and Bacteroides in the ethanol-model were increased significantly $(7.89 \pm$ 0.45 vs. $6.46 \pm 0.29 ; 8.03 \pm 0.15$ vs. $7.38 \pm 0.35, p<0.05)$, and further increased in the ethanol + iron group $(8.90 \pm 0.10 ; 8.28 \pm 0.21, p<0.05)$. However, Lactobacillus, Bifidobacterium, and Clostridium tender were remarkably decreasing in the ethanol-model and iron-model compared with the control group, and further decreased in the ethanol + iron group $(6.92 \pm 0.15 / 6.97 \pm 0.48$ vs. $8.13 \pm 0.44 ; 3.65 \pm 0.22 / 3.78$ \pm 0.19 vs. $4.58 \pm 0.48 ; 6.89 \pm 0.16 / 6.77 \pm 0.38$ vs. $7.66 \pm 0.19, p<0.05)$. With the pretreatment of Aplysin, Bifidobacterium and Clostridium tender significantly increased but Escherichia coli decreased relative to the three model groups $(p<0.05)$. Aplysin exhibited a regulatory and protective effect on intestinal flora disorders induced by ethanol and iron exposure in rats.

\subsection{Nutrient Intakes and Adherence to Dietary Guidelines in Haemodialysis Patients in New Zealand: Room for Improvement}

\section{Z. Xie ${ }^{1}$, R. M. McLean ${ }^{2}$ and M. R. Marshall ${ }^{3}$}

1 Dept Human Nutrition, University of Otago, New Zealand

2 Dept Preventive and Social Medicine, University of Otago, New Zealand

3 School of Medicine, University of Auckland, Department of Renal Medicine, Counties Manukau District Health Board, Auckland, New Zealand, Baxter Healthcare (Asia) Pte Ltd., Singapore, Singapore

Patients undergoing haemodialysis have a high rate of mortality, mostly due to cardiovascular disease. Current renal-specific guidelines contain a number of nutrient recommendations including the restriction of dietary sodium. This observational study describes baseline nutrient intakes in a multi-ethnic sample of 85 adult haemodialysis patients participating in the SoLID Trial in New Zealand. Three-day weighed food records were analysed using Foodworks 8 Professional food composition database, supplemented by other sources of nutrient information. Sixty-six percent of participants were male, with a mean (SD) age of $52 \pm 13$ years. The largest ethnic group was 'New Zealand European and Others' (45\%), followed by 'Pacific' $(29 \%)$. One third of participants had a diagnosis of diabetes mellitus. Mean (SD) sodium intake was $2502 \pm 957 \mathrm{mg} /$ day $(2591 \mathrm{mg} /$ day for men and $2330 \mathrm{mg} /$ day for women). Only $24 \%$ of participants met the recommended level of sodium intake, with $54 \%$ exceeding the recommended sodium intake range. Sodium intake was positively associated with energy intake $(p<0.001)$. Protein intake contributed around $18 \%$ of total energy. Saturated fat intake contributed a mean of $13 \%$ total energy intake, and mean carbohydrate intake accounted for $47 \%$ of total energy. About two thirds of participants were consuming inadequate fibre 
and around half of participants reported excess phosphorus intake. A high proportion of dialysis patients in the SoLID Trial did not meet current renal-specific dietary recommendations for a range of nutrients. A total diet approach is needed to lower sodium intake and improve total diet quality among haemodialysis patients in New Zealand.

\subsection{Nutritional Risks, Undernutrition, and Nutrition Support among with Chronic Kidney Disease in a Tertiary Hospital in Shenzhen}

Hengjiao Chen 1, Qian Sun 2, Ming Zhang 1, Li Xin 1, Yang Wang ${ }^{3}$, Xiaomeng Li ${ }^{3}$, Kang Yu ${ }^{4}$ and Zhuming Jiang ${ }^{4}$

1 Peking University Shenzhen Hospital, China

2 Qingdao Ninth People Hospital, China

3 FUWAI Hospital, China

4 Peking Union Medical College Hospital, China

Background: This study aimed to investigate the prevalence of nutritional risk, undernutrition, and nutritional support among inpatients with chronic kidney disease in a hospital in Shenzhen. Methods: A total of 1293 inpatients who were treated in the department of nephrology were consecutively recorded from April 2015 to September 2016. After 20 patients were excluded for hospital length of stay less than $24 \mathrm{~h}$, or age less than 18 years or more than 90 years, 1273 patients were sampled. After patients who did not meet the scheduled diagnosis were excluded, 1176 patients with chronic kindney disease were enrolled. Nutritional Risk Screening tool (NRS-2002) was performed within the first $24-48 \mathrm{~h}$ after admission and nutrition support was evaluated for 5-14 days. Results: A total of 1176 patients with chronic kindney disease met the inclusion criteria. The prevalence of nutritional risk of chronic kindney disease, chronic glomerulonephritis, nephritic syndrome, and chronic renal failure was 33.1\% (389/1176), 26.3\% (131/499), 18.7\% (34/182), and 46.8\% (256/547), respectively. The undernutrition rate of inpatients with chronic kindney disease was $4.5 \%$ (69/1172), 5.0\% (27/545), 2.7\% (5/182), 8.6\% (47/547), if evaluated from the score of nutrition defect part of NRS-2002, and 9.7\% (127/1172), 11.6\% (63/544) 7.1\% (13/182), 13.0\% (71/547) if based on body mass index $(\mathrm{BMI})<18.5 \mathrm{~kg} / \mathrm{m}^{2}$. In total, $16(4.1 \%(16 / 389)$ inpatients with nutritional risk received nutrition support with the energy and intake being $21.4 \pm 3.6 \mathrm{kcal} / \mathrm{kg} \cdot$ day and $0.87 \pm 0.15 \mathrm{~g} / \mathrm{kg} \cdot \mathrm{day}$, respectively. Of the 787 patients without nutritional risk, four $(0.5 \%)$ received nutrition support. Conclusion: The rate of nutritional risk was high in patients with chronic kindney disease. However, the undernutrition rate was low and the nutrition support rate with nutritional risk was very low. The energy and nitrogen of enteral nutrition support was lower.

\subsection{Relation of Leptin and Inflammatory Markers to Vitamin D Status in Active Pulmonary Tuberculosis}

Q. Z. Wang, ${ }^{1}$, Y. Ma ${ }^{2}$ and Y. F. Liu ${ }^{3}$

1 Institute of Human Nutrition, Medical College of Qingdao University, Qingdao, China

2 Institute of Human Nutrition, Medical College of Qingdao University, Qingdao, China

3 Qingdao Chest Hospital, Chongqing Middle Road, Qingdao, China

Deficient or inadequate vitamin $\mathrm{D}(\mathrm{VD})$ levels are common in active tuberculosis (TB) and may be implicated in the etiology of inflammation and impaired immunity. The aim of this study was to investigate the association between VD status and markers of inflammation levels and leptin in TB patients. Two hundred and ninety-nine active TB patients aged 18 65 years were recruited from Qingdao TB clinics from October 2015 to August 2016. Information on sociodemographics, dietary intake, and living habit of the participants was obtained. Fasting blood samples were collected to measure serum $25(\mathrm{OH}) \mathrm{D}$, leptin, TNF- $\alpha$, IL-6, and C-rective protein (CRP). Multivariate liner regression was used for correlation analysis. Leptin concentrations were higher in individuals in the high VD group than the low VD group, while TNF-a levels were lower in the former $(p<0.05)$. Serum $25(\mathrm{OH}) \mathrm{D}$ concentrations were positively correlated with leptin levels $(p<$ $0.05)$. Further stratified analysis showed that VD status was a significant predictor of leptin and TNF-a in TB patients without Diabetes Mellitus. Meanwhile, in TB patients with DM, a negative 
correlation existed between VD and CRP $(p<0.033)$. VD status was negatively correlated with inflammatory markers and positively correlated with leptin levels in active TB patients. These findings suggest that an adequate VD status may be required for optimal treatment for TB.

\subsection{Nutrition Status Changes in Breast Cancer Patients Who Receive Continous Nutrition Counseling in the Radiotherapy Unit at Dr. Sardjito Hospital, Indonesia}

Susetyowati ${ }^{1, *}$, Dwidanarti Sri Retna ${ }^{2}$, Pangastuti Retno ${ }^{2}$ and Wulandari Hanifah ${ }^{1}$

1 Department of Health and Nutrition, Faculty of Medicine, Universitas Gadjah Mada, Farmako street, Sekip Utara Yogyakarta 55281, Indonesia

2 Sardjito Hospital, Kesehatan street number 1, Yogyakarta 55281, Indonesia

* Correspondence: susetyowati@ugm.ac.id

(1) Background: Counseling strategies in breast cancer patients show that long-term adherence to a dietary pattern is a challenge in studies on the relationship between diet and disease. The aim of this study is to show the effects of nutrition counseling to improve the nutrient intake and nutritional status of breast cancer patients in the radiotherapy unit at Dr. Sardjito Hospital; (2) Methods: A preexperimental study was conducted with one group pretest-posttest design. The subjects included 45 breast cancer patients who underwent radiotherapy for five weeks. Continuous nutrition counseling was given in the first week, third week, and fifth week of radiotherapy. The parameters of nutritional status were anthropometric, biochemical, physical, and dietary, in addition to a patient-generated subjective global assessment (PG-SGA) questionnaire. (3) Results: In the beginning of the study, most of patients had intakes of protein, carbohydrates, and vitamins $\mathrm{A}, \mathrm{C}$, and $\mathrm{E}$ below the recommended dietary allowance (RDA) $(68.9 \% ; 55.6 \% ; 82.2 \% ; 57.8 \% ; 100 \%$, respectively), with a body mass index indicative of obesity $\left(>25 \mathrm{~kg} / \mathrm{m}^{2}\right)(44.4 \%)$. At the end of the study, increased intakes of energy $(124.54$ $\mathrm{kcal})$, protein $(8.12 \mathrm{~g})$, carbohydrates $(5.84 \mathrm{~g})$, vitamin A $(234.43 \mathrm{mg})$, vitamin C (0.042 $\mathrm{mg})$, and vitamin E $(0.44 \mathrm{mg})$ were exhibited, as well as increased hand-grip strength and PG-SGA. Also, decreased body weight $(0.28 \mathrm{~kg})$ was observed, but no changes in hemoglobin and albumin levels were noted. No statistic significance was achieved in any of the parameters, except protein intake ( $p$ $=0.01$ ). (4) Conclusion: Nutrition counseling for breast cancer patients is important to maintain and improve nutrition intake and nutritional status. In the long-term period, it might improve quality of life.

Keywords: nutrition status; breast cancer; nutrition counseling; radiotherapy

\subsection{Decomposition of Changes in the Consumption of Macronutrients in Vietnam between 2004 and 2014}

Trinh Huong Thi ${ }^{1}$, Simioni Michel ${ }^{2}$ and Thomas-Agnan Christine ${ }^{3}$

1 Toulouse School of Economics, INRA, University of Toulouse Capitole, Toulouse, France

2 INRA, UMR-1110 MOISA, Montpellier, France

3 Toulouse School of Economics, University of Toulouse Capitole, Toulouse, France

Vietnam is undergoing a nutritional transition, like many middle-income countries. This paper proposes to highlight the sociodemographic drivers of this transition over the period 2004-2014. A method of decomposition of between-year differences in economic outcomes recently proposed in the literature is implemented. The common objective of decomposition methods is to decompose between-group differences into two components: a composition effect due to differences in observable covariates across groups, and a structure effect due to differences in the relationship that links the covariates to the considered outcome. This method also allows us to decompose the composition effect, which is due to the differences in observable covariates across years, in the direct contribution of each of the covariates and the effect of their interactions. This method is applied to the "Vietnam Household Living Standard Survey", or VHLSS data. The results show the importance of composition effect and therefore of between-year changes in the distributions of covariates on between-year changes in the distributions of per capita calorie intake and per capita calorie intakes from proteins and fats. This effect is more contrasted in the case of per capita calorie intake from 
carbohydrates. Food expenditure and household size appear to be the main drivers of the observed evolutions in macronutrients consumption.

\subsection{How Should We Regulate Dietary Supplements?}

M. K. Lee ${ }^{1}$ and C. W. Binns ${ }^{2}$

1 School of Health Sciences, Murdoch University, Perth, WA, USA

2 School of Public Health, Curtin University, Perth, WA, USA

Dietary supplements have grown into a massive global business worth more than $\$ 100$ billion annually and are projected to double in value in the next decade. They are taken by up to $50 \%$ of adults and perhaps one-third of children in the economically-advanced economies. Definitions of dietary supplements differ from country to country and over time, making comparisons between jurisdictions and time series difficult to interpret. This review discusses the public health issues associated with dietary supplements and suggests directions for further regulation. An extensive literature search was carried out in PubMed, spanning the past 10 years and including regulatory authorities in selected countries (US, Canada, Australia, European Union, China, Japan, and Korea). The risks of using dietary supplements include organ damage from inherent toxicity, interactions, or product contamination. The monitoring and evaluation of supplements is difficult since many contain multiple ingredients, change composition over time, or are used intermittently at doses which are difficult to measure. Regulation is generally lax in most countries, as there are more than 100,000 products on the market, and often seems to be more directed towards promoting commerce than protecting public health.

In general, dietary supplements are not needed except in cases of established deficiencies and prevention needs, and excess of some nutrients is associated with increased cancer rates and shortened life expectancy. A high standard of premarketing regulation would be costly, and governments are reluctant to implement these changes. Improved regulatory requirements for dietary supplements are needed to ensure public safety.

\subsection{Fish, Long Chain n-3 PUFA Consumption, and Risk of All-Cause Mortality: A Systematic Review and Meta-Analysis}

\section{Y. Wan ${ }^{1}$, J. Zheng ${ }^{2}$, F. Wang ${ }^{1}$ and D. Li ${ }^{1,3}$}

1 Department of Food Science and Nutrition, Zhejiang University, Hangzhou, China

2 MRC Epidemiology Unit, University of Cambridge, Cambridge, UK

3 Institute of Nutrition and Health, Qingdao University, Qingdao, China

The consumption of fish and long-chain omega-3 polyunsaturated fatty acids (n-3 PUFAs) may influence the risk of all-cause mortality, but research findings have been inconsistent. The current systematic review and meta-analysis aims to clarify the association between fish and long-chain n-3 PUFA consumption with risk of all-cause mortality. Studies published before March 2017 were identified through electronic searches using PubMed, Scopus, and Web of Science databases. We included prospective cohort studies that reported relative risks with $95 \% \mathrm{CI}$ of all-cause mortality for fish and long-chain n-3 PUFA consumption. Results were combined using a random effects model. Twenty-three prospective cohorts with a total of 1,035,416 participants were included. Twenty-two pooled studies involving 985,126 participants indicated that fish intake was associated with a $6 \%$ (RR: 0.94; 95\% CI: $0.90,0.98)$ reduction in the risk of all-cause mortality. Six studies with 430,579 participants investigated the association between long-chain n-3 PUFAs and all-cause mortality risk; the relative risk for highest versus lowest categories was 0.86 (95\% CI: 0.80, 0.93). Dose-response analysis suggested that the risk of all-cause mortality was reduced by $7 \%$ (RR: $0.93 ; 95 \%$ CI: $0.88,0.99$ ) for every 0.2-g-per-day increment in long-chain n-3 PUFA consumption. The current meta-analysis indicates that both fish and long-chain n-3 PUFA consumption are inversely associated with risk of all-cause mortality. These findings could have public health implications with regard to lowering the risk of all-cause mortality through dietary interventions. 


\subsection{Lack of Relationship between Chromium and Blood Glucose Concentration in the Chromium-Deficient} and Free-Living Populations

A. Chu ${ }^{1}$, R. Alhazzaa ${ }^{2}$, P. Varelis ${ }^{2}$, D. Mackerras ${ }^{2}$ and S. Samman ${ }^{1,3}$

1 Department of Human Nutrition, University of Otago, Otago, New Zealand

2 Food Standards Australia New Zealand Canberra, ACT

${ }^{3}$ School of Life and Environmental Sciences, University of Sydney, Sydney, NSW

Chromium has been associated with the maintenance of normal blood glucose levels in chromium-deficient and healthy populations. However, no previous systematic review has summarised the effect of chromium on glycaemic control in the chromium-deficient population. The aim of this systematic review was to determine the relationship between chromium and blood glucose concentration in chromium-deficient and free-living populations. Five studies were included in the chromium-deficient population, and chromium was given intravenously in all included studies. The form and dose of chromium administration cannot be extrapolated to represent a foodhealth relationship. Therefore, we considered the relationship between dietary chromium and blood glucose levels in a chromium-deficient population to be not assessable. In the free-living population, an update of a previous systematic review was conducted. In Balk et al.'s review (Diabetes Care, 2007; 30:2154-63), 19 randomised controlled trials (RCTs) (22 strata) in normoglycaemic populations and 7 RCTs (9 strata) in impaired glucose tolerance populations were included. In the update, we identified three new relevant studies: two studies conducted in adults with impaired glucose tolerance, and another in normoglycaemic overweight children. Meta-analyses in normoglyacemic and impaired glucose tolerance populations indicate no effect of chromium on blood glucose levels. No serious concerns of study biases, inconsistency, imprecision, or other methodological issues were identified; however, the current evidence is limited by small sample sizes. Therefore, we conclude that there is a moderate degree of certainty for no relationship between chromium intake and blood glucose levels in both normoglycaemic and impaired glucose tolerance populations.

\subsection{Prevention of Prostate Cancer Stem Cells (CSCs) by Bowman-Birk Inhibitor (BBI) from Soybean}

\section{S. Kaneko, C. Sato and T. Yano}

Graduate school of Food and Nutrition Science, Toyo University, Gunma, Japan

Soybean is known as a food having a preventive effect on prostate cancer. The most predominant protease inhibitor in soybeans is the Bowman-Birk inhibitor (BBI), which is a well-established cancer chemopreventive agent due to its strong anticarcinogenic activity. Prostate cancer is increasing in Japan year by year. Recently, the existence of cancer stem cells (CSCs) in cancer tissues and cell lines has been reported. It is assumed that CSCs are a main cause of oncogenesis and recurrent cancer. Our previous study has shown that negative growth control in cancer cells by the BBI partly depends on the induction of connex43 (Cx43), a tumor suppressor gene. In this study, we show a growth regulation of prostate CSCs by BBI. LNCaP cells (androgen-dependent prostate cancer) were used. Then, we made spheroid-formed CSCs by LNCaP. Cell viability was evaluated by a WST-1 assay. The level of mRNA was determined by real time polymerase chain reaction (RT-PCR). We evaluated the combined effect of BBI and docetaxel (an anti-cancer agent) by a WST-1 assay. BBI effectively inhibited prostate cancer cell growth in a dose-dependent manner $(0-400 \mathrm{~g} / \mathrm{mL} 0.01)$. The level of Cx43 mRNA was increased by BBI treatment with a statistical significance. In parallel with BBIinduced Cx43, some markers of CSCs were decreased (0.01). In addition, BBI drastically cancelled the expression of androgen receptor, androgen independency, and resistance to anticancer agents in CSCs. The BBI drastically changed the characteristics of prostate cancer stem cells. This effect of BBI on CSCs may lead to the establishment of a new effective therapy against prostate cancer.

\subsection{Second Voided Early Morning Urine Minerals Changed Significantly with or without Sodium Restriction in Humans}

N. Serizawa 1,2, M. Nishimuta 1,3, N. Kodama 2,3, M. Shimada 3,4 and Y. Yoshitake ${ }^{3,5}$ 
Toyo University, Japan

Tokyo shokuryo dietitian academy, Japan

Nat'1 Inst. of Health and Nutrition, Japan

Chiba Pref. Univ. Health Sci, Japan

Nat'1 Inst. of Fitness and Sports in Kanoya, Japan

We consider the second voided early morning urine (EMU) as possible indicators for the status of mineral nutrition.

We already reported the weak but significant positive correlation between dietary sodium $(\mathrm{Na})$ intakes and the EMU-Na in human mineral balance studies. In this experiment, we examined the EMU contents of minerals ( $\mathrm{Na}, \mathrm{K}, \mathrm{Ca}, \mathrm{Mg}, \mathrm{P}$, and $\mathrm{Zn}$ ) with and without Na restriction (6 g/d or $12 \mathrm{~g} / \mathrm{d}$ as $\mathrm{NaCl}$ ) under a diet each for the balance study.

The result showed that under the low Na-intake (6 g/d), the EMU-Na, Ca, Mg, P, and Zn showed lower values than the control $(12 \mathrm{~g} / \mathrm{d})$. However, no significant difference in the EMU-K was observed between the low-Na diet and the control diet.

Under the low-Na diet, compared to the control diet, the excretion of $\mathrm{Na}, \mathrm{Ca}, \mathrm{Mg}, \mathrm{P}$, and $\mathrm{Zn}$ in the EMU was significantly lower, although the mineral intakes except $\mathrm{Na}$ were unchanged. We therefore consider that the excretion of $\mathrm{Na}, \mathrm{Ca}, \mathrm{Mg}, \mathrm{P}$, and $\mathrm{Zn}$ in the EMU reflects the low-Na diet.

3.70. Nutrition Knowledge of Dietary Iron and Awareness of Iron Education in Reproductive-Age Women in New Zealand

\section{P. E. Meads and M. A. Choukri}

Department of Nursing, Midwifery \& Allied Health, Ara Institute of Canterbury, Christchurch, New Zealand

Iron education is a method used worldwide by governments and health organisations to highlight the importance of iron in the daily diet. Several studies have reported that effective nutrition education and information regarding iron is essential to addressing iron deficiency, and one study suggests that educating women about increasing the frequency of meat consumption, knowledge of iron-rich foods, and enhancers and inhibitors of dietary iron could improve iron status. At present, there is limited research examining the relationship between iron education awareness and the level of nutrition knowledge of iron in reproductive-age women living in New Zealand. We recruited 100 women aged 20-40 years in Christchurch to complete a questionnaire on dietary habits, dietary iron nutrition knowledge, and iron education awareness. Results from this study will be available in September 2017, and will provide insight into what women know about iron and where they obtain information about iron. Results could help to inform future education approaches and strategies, with the ultimate goal of increasing the daily intake of iron in women of childbearing age.

\subsection{Seasonal Variation in the Phytochemical Content of the Australian Prickly Pear (Opuntia ficus indica)}

C. A. Gouws 1,2, N. D'Cunha 1,2, E. N. Georgousopoulou 1,2, J. Thomas 1,2, D. Mellor 1,2,3 and N. Naumovski ${ }^{1,2}$

Faculty of Health, University of Canberra, Canberra 2617, Australia

The Prickly Pear (PP) fruit (Opuntia ficus indica) has been used in traditional medicine, and is commonly eaten fresh. Its phytochemical composition is influenced by seasonal variation. This study aimed to determine the antioxidant and bioactive content of Australian commercially-grown PP between harvesting seasons. White, orange, and purple PP fruit skin and flesh were dried using microwave drying, followed by methanolic extraction. Antioxidant characteristics, free radical scavenging activity (2,2-Diphenyl-1-Picrylhydrazyl (DPPH)), reducing capacity (CUPRAC), and antioxidant capacity (ferric reducing activity of plasma (FRAP)) were expressed as Trolox equivalents (TE). Total phenolic (Folin-Ciocalteu; gallic acid equiv. (GAE)), flavanol ( $\mathrm{AlCl}_{3}$; Catechin equiv. (CE)), betalain (betaxanthin (indicaxanthin eqiv. (IE)), and betacyanin (betanin equiv. (BE)) contents were also determined. The differences between harvests were determined using the Student's $t$-test, where statistical significance was set at $p<0.05$ and results were represented as 2015 vs. 2016. The 2015 PP 
harvest was higher in antioxidant characteristics for white flesh CUPRAC (3189 \pm 292 MTE vs. $1399 \pm$ $283 \mathrm{M}$ те; $p=0.002)$, FRAP white flesh $(1499 \pm 102 \mathrm{M}$ те vs. $630 \pm 121 \mathrm{M}$ те; $p=0.001)$, white skin $(1417 \pm$ 8.73M Me vs. $\left.847 \pm 101 \mathrm{M}_{\mathrm{TE}} ; p=0.001\right)$, and orange skin $(1473 \pm 146 \mathrm{M}$ TE vs. $763 \pm 40.9 \mathrm{M}$ те; $p=0.01)$. Orange skin was higher in GAE $(84.5 \pm 34.73$ gGAE vs. $75.6 \pm 31.9$ gGAE; $p=0.004)$. Only Purple PP skin of the 2016 harvest was higher in DPPH $(163 \pm 48.9 \mathrm{M}$ те vs. $258 \pm 15.2 \mathrm{M}$ те; $p=0.032)$ and FRAP $(1558 \pm$ $0.001 \mathrm{M}$ te vs. $900 \pm 146 \mathrm{M}$ те; $p=0.001)$, as well as CE $(86.6 \pm 3.25$ gCE vs. $171 \pm 6.23$ gCE; $p<0.001)$, BE $\left(1.43 \pm 0.05\right.$ mgBE vs. $\left.2.40 \pm 0.39 \mathrm{mg}_{\mathrm{BE}} ; p=0.048\right)$, and IE $\left(1.19 \pm 0.12 \mathrm{mgIE}\right.$ vs. $\left.2.04 \pm 0.19 \mathrm{mg}_{\mathrm{IE}} ; p=0.003\right)$ content. Variation in content was observed between harvests. This could be due to the plants' protective mechanisms towards changes in environmental conditions, and should be considered during nutraceutical preparations.

\subsection{Association of Dietary Selenium Intakes and Metabolic Syndrome among Malaysian Adults in Klang Valley}

\section{S. S. Tan, C. K. M. Lim, N. H. Jackney, L. S. Ban and E. H. Lau}

Department of Nutrition and Dietetics, School of Health Sciences, International Medical University, 57000 Kuala Lumpur, Malaysia

Metabolic syndrome (MetS) is a major global disease that represents of abdominal obesity, insulin resistance, hypertension, and dyslipidemia. Selenium is an essential trace element found to protect our body's cells against reactive oxygen species and oxidative stress. Dietary and lifestyle factors may contribute to increased risk of developing MetS and oxidative stress. Low antioxidant status may be associated with the unfavourable metabolic pattern. However, currently available data are inconsistent. This study aimed to determine the association of dietary selenium intake and MetS among Malaysian adults aged 20-55 years old in the Klang Valley. A total of 98 participants were recruited for this cross-sectional study. Anthropometry profiles were measured from subjects. Fasting blood glucose (FBG) and lipids were determined via finger prick method after at least $8 \mathrm{~h}$ of fasting. Blood pressure (BP) measurement was taken using a digital blood pressure monitor. A food frequency questionnaire was used to determine the dietary selenium intake of the study population. All data were analysed using the Statistical Package for the Social Sciences version 20. The mean dietary selenium intake of the study population was $62.16 \pm 32.93 \mu \mathrm{g} /$ day. There were no significant correlations between dietary selenium intake and BMI $(\mathrm{r}=0.011, p=0.913)$; waist to hip ratio (WHR) $(\mathrm{r}=0.018, p=0.860)$; body fat $(\mathrm{r}=0.118, p=0.246)$; fasting blood glucose (FBG) $(\mathrm{r}=0.040, p=0.696)$; total cholesterol $(\mathrm{r}=0.116, p=0.257)$; triglyceride $(\mathrm{r}=0.076, p=0.457)$; low density lipoproteincholesterol (LDL-c) $(\mathrm{r}=0.092, p=0.366)$; high density lipoprotein-cholesterol (HDL-c) $(\mathrm{r}=-0.013, p=$ 0.898); or systolic $(\mathrm{r}=0.104, p=0.312)$ and diastolic $(\mathrm{r}=-0.016, p=0.874)$ BP. Current findings suggested that the dietary selenium intake and MetS were not associated among the Klang Valley population of Malaysia. Further longitudinal studies are required to obtain a better understanding of the association between the variables.

\subsection{The Combination of Vitamin E Homolog-Induced Cytotoxicity on Prostate Cancer Cells}

\section{Sato, M. Ota and T. Yano}

Graduate school of Food Life science, Toyo University, Itakura, Gunma, Japan

Vitamin E homologs are known as anti-cancer ingredients with high safety. The epidemiological analysis of vitamin $\mathrm{E}$ intake has indicated a prominent preventive effect against prostate cancer (PC). Therefore, we investigated whether the combination of $\gamma$-Toc with $\delta$-T3 could strengthen the inhibitory effect of $\delta$-T3 on prostate cancer cell growth. Cell viability was evaluated by WST-1 assay. Cell cycle and apoptosis population analysis was performed by the cell analyzer. Additionally, each cyclin expression level was carried out by Western blot analysis. Statistical analyses were performed by the Tukey-Kramer test. The combination treatment ( $\delta$-T3 $10 \mathrm{~g} / \mathrm{mL}, \gamma$-Toc $10 \mathrm{~g} / \mathrm{mL}$ ) showed a significant cytotoxic effect on PC cells $(p<0.01)$. Specifically, cell cycle phase distribution analysis revealed that in addition to $G 1$ arrest caused by the treatment with $\delta$-T3, the combination of $\delta$-T3 with 
$\gamma$-Toc induced G2/M arrest. These observations were completely confirmed by the results that $\delta$-T3 induced a reduction of cyclin D1 level, $\gamma$-Toc reduced cyclin B1 level, and the combination simultaneously decreased the level of two cyclins. Enhanced induction of apoptosis by the combined treatment was also observed. These findings indicate that the combination of $\delta$-T3 and $\gamma$-Toc significantly inhibits prostate cancer cell growth due to the simultaneous cell cycle arrest in G1 phase and G2/M phase. These results suggest that the combination treatment of $\delta$-T3 and $\gamma$-Toc effectively induces cytotoxicity on androgen-independent PC cells in comparison to the single treatment of $\delta$ T3 or $\gamma$-Toc.

\subsection{The Effect of Folate Supplementation on Inflammatory Markers in Depression: A Systematic Review}

H. M. Barnett 1,2, N. M. D'Cunha 1,2, E. N. Georgousopoulou 1,2,3, D. D. Mellor 1,2,4, A. J. McKune 1,2,5, J. Kellett ${ }^{1,2,5}$ and N. Naumovski ${ }^{1,2,5}$

1 Faculty of Health, University of Canberra, Canberra, ACT, Australia

2 Collaborative Research in Bioactives and Biomarkers (CRIBB) Group, University of Canberra, Canberra, ACT, Australia

3 Department of Nutrition-Dietetics, School of Health and Education, Harokopio University, Athens, Greece

4 School of Life Sciences, Coventry University, Coventry, UK

5 University of Canberra Health Research Institute (UCHRI), University of Canberra, Bruce, Canberra, ACT, Australia

Folate has been implicated in the pathogenesis of depression, yet the mechanism is not clearly understood. The importance of folate in the human diet has resulted in the mandatory fortification of flour with folic acid (FA) in over 80 countries. However, as FA is a synthetic form of folate, there is increasing concern that FA may have the potential to induce epigenetic changes linked with an increased risk of inflammation-related non-communicable diseases (e.g., cardiovascular disease, type 2 diabetes, depression). Therefore, following the PRISMA 2009 guidelines, we conducted a systematic review of electronic databases (PUBMED, Scopus, CINAHL, and Cochrane Library) to identify all human clinical trials examining the effects of folate (including FA) supplementation in the management or prevention of depression. We also sought to determine the types of folate supplementation used, the impact on inflammatory markers (Hcy, interleukins, tumor necrosis factor-alpha and high-sensitivity C-reactive protein) and if genetic polymorphisms were considered. Ten trials met the inclusion criteria. Six trials examined effects of either adjunctive FA or Lmethylfolate supplementation with antidepressants in the management of depression, three examined the effects of FA supplementation alone for the prevention of depression, and one trial included participants taking antidepressant medication throughout the trial period, although antidepressant therapy was not part of the intervention. No evidence was found to support FA supplementation for the management or prevention of depression. More research is required to determine the efficacy of L-methylfolate in certain populations and the significance of certain genetic polymorphisms.

\subsection{Adherence to Australian Dietary Guidelines: Students' Experiences Following an Individualised Diet} Plan as Part of an Undergraduate Nutrition Course

C. Irwin ${ }^{1}$, B. Desbrow ${ }^{1}$, S. Khalesi ${ }^{2}$ and D. McCartney ${ }^{1}$

1 Menzies Health Institute Queensland, School of Allied Health Sciences, Griffith University, Gold Coast, Australia

2 School of Health, Medical and Applied Sciences, Central Queensland University, Rockhampton, Australia

Food-based dietary guidelines are designed to support the population in adopting a healthy and balanced diet. University students are notorious for exhibiting poor eating behaviors and are a population group at risk of not meeting dietary guidelines. The aim of this study was to: (1) compare the three-day "usual" dietary intake of university students enrolled in a nutrition course against the Australian Dietary Guidelines (ADGs) and Nutrient Reference Values (NRVs); and (2) explore students' experiences in following a three-day individualised diet plan adhering to the ADGs/NRVs, 
including potential enhancers and inhibitors of adherence. Overall, students' usual Food Group and nutrient intakes deviated widely from the guidelines, with few dietary components consumed in recommended quantities. Several key challenges and enablers to dietary adherence were identified when following the planned diet. These were commonly categorised as personal/behavioral factors (e.g., the quantity/type of food) and societal factors (e.g., time, cost, social factors). Findings from this study provide insight into challenges and enablers that may affect adherence to dietary guidelines in this population group. This study identifies a need for more educational resources to support this population group, translating the ADGs and NRVs into examples of meals and/or dietary patterns. This study also highlights a need for further research to evaluate consumers' ability to use/apply the ADGs. Given widespread concerns regarding the poor eating behaviors of university students, researchers and health professionals can use the findings from this study to help tailor advice to facilitate improvement in dietary patterns.

3.76. Potential Influences of Geographical Positioning on Phytochemical Characteristics of Australian-Grown Prickly Pear (Opuntia ficus indica)

C. Gouws ${ }^{1,2}$, N. M. D'Cunha 1,2, E. N. Georgousopoulou ${ }^{1,2,3}$, D. D. Mellor ${ }^{1,2,4}$ and N. Naumovski 1,2,5

1 Faculty of Health, University of Canberra, Canberra, ACT, Australia

2 Collaborative Research in Bioactives and Biomarkers (CRIBB) Group, University of Canberra, Canberra, ACT, Australia

3 Department of Nutrition-Dietetics, School of Health and Education, Harokopio University, Athens, Greece

4 School of Life Sciences, Coventry University, Coventry, UK

5 University of Canberra Health Research Institute (UCHRI), University of Canberra, Bruce, Canberra, ACT, Australia

The Prickly Pear (Opuntia ficus indica) (PP) fruit is a seasonal drought-resistant crop, often used for its beneficial health properties. This study examines the antioxidant and bioactive characteristic variation between PP fruit from selected Australian locations. PP samples (white, orange, and purple) were collected from three sites: two commercially grown (NSW and VIC) and one non-farmed region (ACT). Samples were microwave-dried (adapted AOAC method). Dried samples were analysed for antioxidant characteristics: free radical scavenging activity (2,2-Diphenyl-1-Picrylhydrazyl, DPPH), reducing capacity (CUPRAC), and antioxidant capacity (ferric reducing activity of plasma (FRAP)) (Trolox Equivalents). Bioactive compounds, total phenolic content (Folin-Ciocalteu; gallic acid equivalent (GAE)), total flavanol content $\left(\mathrm{AlCl}_{3}\right.$; catechin eq. (CE)), and total betalain (betaxanthin; indicaxanthin eq. (IE)) and betacyanin (betanin eq. (BE)) contents were also examined. Statistical analysis was performed, and results are expressed as mean \pm SE. Non-farmed fruits (ACT) were observed to contain the greatest DPPH content (purple skin; $851.44 \pm 191.62 \mathrm{MTE}$ ) and other bioactives; total phenolic content (white flesh; $166.16 \pm 14.29 \mathrm{gGAE}$ ); total betalain content (purple skin; $11.46 \pm$ $2.75 \mathrm{mgBE}$ ), more specifically, individual betalains; betacyanin (purple skin; $14.53 \pm 1.30 \mathrm{mg} \mathrm{gE}_{\mathrm{BE}}$; and total betaxanthin content (purple skin; $5.18 \pm 0.58 \mathrm{mg}$ IE). Commercially-grown fruit from VIC contained the highest values for CUPRAC (purple flesh; $3359.00 \pm 529.16 \mathrm{MTE}$ ) and FRAP (purple skin; $919.18 \pm 529.16 \mathrm{MTE})$. Separately, commercially-grown fruit from NSW were observed to contain the greatest total flavonoid content (white skin; $174.89 \pm 57.24 \mathrm{~g}_{\mathrm{CE}}$ ). Phytochemical variation was observed between different PP fruit colours, fruit components, and harvest locations, which could be attributed to farming methods, variations in geographical location, and the plants' protective mechanisms as a result of potential environmental stressors.

\subsection{Excess Iron-Induced Membrane Fluidity Decrease and Component Change of Red Blood Cells in Rats}

A. Ma ${ }^{1, *}$, J. Lv ${ }^{1}$, S. Lin ${ }^{1}$, Y. Wang ${ }^{1}$, H. Zhang ${ }^{1}$, Y. Sun ${ }^{1}$, E. Schouten ${ }^{2}$ and F. Kok ${ }^{2}$

1 The Institute of Human Nutrition, Medical College of Qingdao University, Qingdao, China

2 Division of Human Nutrition, Wageningen University, Wageningen, The Netherlands

* Corresponding Author 
Research on the effect of iron overload on RBCs is relatively scarce. This study was designed to detect the effect of excess iron on erythrocyte membrane composition and function in rats. Forty Wistar male rats were randomly divided into four groups treated with iron dextran by intraperitoneal injection every other day. Group DI (iron deficiency) received $0.3 \mathrm{mg}$ iron, $0.9 \mathrm{mg}$ for group C as a normal; $9 \mathrm{mg}$ and $18 \mathrm{mg}$ were administered to groups $\mathrm{MI}$ and $\mathrm{HI}$, being 10 and 20 times the iron dose of the normal group, respectively. After a 12-week trial, relevant indicators of erythrocyte physiological functions were detected. Lower levels of sulfhydryl (-SH) in rat erythrocyte membranes were found to be $30.1 \%$ and $32.8 \%$ in $\mathrm{MI}$ and $\mathrm{HI}$, respectively, of levels in the control group; while the erythrocyte membrane fluidity was significantly decreased $(p<0.05)$. Meanwhile, the level of sialic acid (SA) and activities of ATPases in the erythrocyte membrane showed decreases in groups with iron excess. SA in $\mathrm{HI}$ decreased by $25.1 \%$ compared with control; activities of $\mathrm{Na}^{+}-\mathrm{K}^{+}$-ATPase and $\mathrm{Ca}^{2+}-\mathrm{Mg}^{2+}$-ATPase decreased by $15.8 \%$ and $16.3 \%$ in the MI group, and decreased by $14.6 \%$ and $19.7 \%$ in HI compared with group DI, respectively. In addition, there was a significant increase of oxidative stress in rats with iron excess in plasma redox status. Excessive iron can result in a decrease of the erythrocyte membrane fluidity, which might be attributed to an increase of oxidative stress and component changes in the erythrocyte membrane.

Acknowledgment: This work was supported by the National Natural Science fund (No. 81373000).

\subsection{Double Burden of Iron Status in Chinese Adult Women with Iron Deficiency and Iron Overload: A Cross-Sectional Study}

Y. Sun ${ }^{1}$, A. Ma ${ }^{1, *}$, X. Ma ${ }^{1}$, S. Lin ${ }^{1}$, Y. Wang ${ }^{2}$, R. Jia ${ }^{1}$, G. Zhang ${ }^{1}$ and Y. Ma ${ }^{1}$

1 Department of Public Health, Medical College of Qingdao University, Qingdao, China

2 Department of Physical Examination Center, Affiliated Hospital of Qingdao University, Qingdao, China

* Corresponding Author

Females face a double burden of iron status: iron deficiency (ID) and iron overload (IO). This study aims to observe the serum ferritin (SF) level and prevalence of ID and IO associated with age vis-à-vis the double burden of ID and IO in adult Chinese women. A total of 2562 women were included in this cross-sectional study from hospitals located in Qingdao, China, between June 2014 and October 2015. Medical examination, biochemical indicators, blood routine examination, serum ferritin $(\mathrm{SF})$, and high-sensitivity C-reactive protein were measured for all participants. The distributions of SF for $36-45 \mathrm{y}, 46-55 \mathrm{y}, 56-65 \mathrm{y}$, and $\geq 66$ y groups were significantly different $(p<$ 0.01). The prevalence of ID (SF $<15 \mu \mathrm{g} / \mathrm{L}$ ) decreased from $27.06 \%$ to $1.59 \%$, while the prevalence of IO $(>150 \mu \mathrm{g} / \mathrm{L})$ increased from $1.15 \%$ to $6.35 \%$, and these two changes had statistical significance $(p<$ 0.01). After adjusting for known risk factors, the odds ratio (OR) of ID was 25.01 (95\% CI 5.98-104.57) for subjects in the 36-45 y group compared with $\geq 66$ y group, while the odds ratio (OR) of IO was 3.21 (95\% CI 1.14-9.01) for subjects in the $\geq 66$ y group compared with $36-45$ y group. These findings confirm an existing double burden of iron status with ID and IO in Chinese adult women, characterized by a high prevalence of ID in premenopausal women, with a high prevalence of IO in postmenopausal women. Development of policy for the management of iron deficiency and iron overload is needed.

Acknowledgments: This work was supported by the National Natural Science fund (No. 81373000).

\subsection{Dietary Intake of Antioxidant Vitamins and Mortality: A Meta-Analysis of 11 Cohort Studies}

Yanyan Kang, Tianlin Gao* and Aiguo Ma *

School of Public Health, Qingdao University, Qingdao 266021, China

* Corresponding Authors

The aim of the study was to assess the relationships between dietary intakes of antioxidant vitamins C, E, and A, active substances (carotenes), and mortality in cohort studies. Relevant Englishlanguage studies were identified though PubMed, Cochrane Library, and EMBASE databases up to July 2017. Multivariate-adjusted risk ratios (RRs) for mortality in the highest verses the lowest 
categories of baseline intake of antioxidant vitamins C, E, and carotenes were pooled using a fixedeffects meta-analysis and reported as relative risk (RR) with $95 \%$ confidence intervals (CIs). Metaregression was used to assess the effect of covariates across the trials. Over the follow-up ranging from 3 to 32 years, 51,277 mortality events occurred among 419,837 adults from 11 cohort studies. Subjects with higher dietary intake of carotenes had a significantly lower risk of mortality in eight studies (pooled RR $=0.85 ; 95 \%$ CI: $0.80,0.91 ; I^{2}=45.2 \%$ ), and those with higher dietary intake of vitamin C had a significantly lower risk of mortality in ten studies $\left(0.88 ; 95 \%\right.$ CI: $\left.0.81,0.95 ; I^{2}=87.5 \%\right)$. The risk of mortality with vitamin E intake was 0.94 (95\% CI: $0.89,1.00 ; I^{2}=45.7 \%$ ) in ten studies. The present finding suggests that increased intake of vitamin $C$ and carotenes in the diet may benefit the prevention of death, but we did not find evidence that higher intake of vitamin $\mathrm{E}$ reduced the risk of death.

\subsection{Elevated Serum Ferritin Level Was Associated with Higher Risk of Hyperuricemia in Chinese Middle- Aged and Elderly Males: A Cross-Sectional Study}

\section{H. Zhang, S. Lin, X. Ma and A. Ma*}

The College of Public Health, Qingdao University, 38 Dengzhou Road, Qingdao 266021, China

* Corresponding Author

Few studies have examined whether serum ferritin level was a risk factor for hyperuricemia (HUA). The objective of this cross-sectional study was to detect the relationship between the serum ferritin level and HUA in Chinese middle-aged and elderly males. A total of 5389 males aged 45 to 91 y were recruited in Qingdao, China. Anthropometric data were collected following general physical examinations. Serum lipids, glucose, inflammatory molecules, ferritin, and uric acid were determined. We found that the highest prevalence of HUA occurred in the highest quartile of serum ferritin. Compared with the non-HUA group, the HUA group had higher serum ferritin levels (median 106.08 vs. $97.88 \mu \mathrm{g} / \mathrm{L} ; p<0.001$ ). The result of Spearman correlation indicated that there was a positive correlation between serum ferritin and serum uric acid. After adjusting for age, alcohol consumption, smoking status, body mass index, systolic blood pressure, diastolic blood pressure, triglycerides, total cholesterol, and blood glucose, the odds radios and $95 \%$ confidence intervals for HUA for the second, third, and fourth quartiles versus the first quartile were 1.150 (0.938-1.410), 1.274 (1.041-1.559), and 1.308 (1.066-1.604), respectively ( $p$-value for trend $=0.003)$. Receiver operating characteristic curve analysis revealed that the area under the curve was 0.555 (95\% CI, $0.530-0.570)$, statistically significant for serum ferritin level association with HUA $(p<0.001)$. In conclusion, the increased serum ferritin concentrations were associated with HUA, and elevated serum ferritin level is an independent risk factor for HUA in Chinese middle-aged and elderly males.

\subsection{Association between Vitamin C and Risk of Hyperuricemia in American Adults}

Yongye Sun, Xueling Xin, Dongfeng Zhang, Jianping Sun and Aiguo Ma

Quingdao University, Shandong, China

Background: Few studies have reported the relationship between vitamin $C$ intake and hyperuricemia among American adults. The aim of the present study was to examine the associations between total vitamin $C$ and dietary vitamin $C$ intakes with the risk of hyperuricemia.

Methods: Data from three 2-year cycles of the National Health and Nutrition Examination Survey (NHANES) 2009-2012 were used in the present study. Dietary intake was obtained through two 24-h dietary recall interviews. Logistic regression models were applied to examine the associations between total vitamin $\mathrm{C}$ and dietary vitamin $\mathrm{C}$ intakes with risk of hyperuricemia.

Results: A total of 14,885 adults aged 18 years or older (7269 men and 7616 women) were included in the present study. The prevalence of hyperuricemia was $19.05 \%$. For all subjects, total vitamin $C$ and dietary vitamin $C$ intakes were inversely associated with risk of hypeuricemia in the unadjusted model and in the age- and gender-adjusted model. The multivariate adjusted odds ratios (ORs) with 95\% confidence intervals (CIs) of hyperuricemia were $0.66(0.58-0.76)$ and $0.72(0.63-0.82)$ 
for the highest versus lowest quartile of total vitamin $C$ and dietary vitamin $C$ intakes, respectively. Analyses stratified by gender were also performed, and similar results were observed in females and males.

Conclusion: This study showed that total vitamin $C$ and dietary vitamin $C$ intakes may be inversely associated with risk of hyperuricemia among American adults. Data from large cohort studies are needed to confirm our findings.

\subsection{Adherence to Mediterranean Diet and LDL-c Levels Are Independent Predictors of Depression in Older Adults: The MEDIS Study}

Ekavi Georgousopoulou, Nathan D'Cunha, Nenad Naumovski, Stefanos Tyrovolas, Suzanne Piscopo, Giuseppe Valacchi, Nikos Tsakountakis, Akis Zeimbekis, Vassiliki Bountziouka, Efthimios Gotsis, George Metallinos, Dimitra Tyrovola, Alexandra Foscolou, Josep A. Tur, Antonia L. Matalas, Christos Lionis, Evangelos Polychronopoulos, Labros S. Sidossis and Demosthenes B. Panagiotakos

Australian National University, Canberra 0200, Australia

Low cholesterol and poor diet are associated with increased risk of depression. We aimed to evaluate the relationship between cholesterol, diet quality, and symptoms of depression in older adults enrolled in the Mediterranean Islands (MEDIS) study between 2004 and 2015. The sample consisted of 2749 individuals of which 1380 were female (F) (mean age: $73.6 \pm 7.14$ years $(y)$ ), and 1369 were male (M) (mean age: $74.8 \pm 7.49$ y). Participants were categorised based on Geriatric Depression Scale (GDS) score, a 30-item measure of symptoms of depression (0-9: normal; 10-19: mild; 20-30: severe). Using the MedDietScore (range $=0-55$ ), the mean level of adherence to a Mediterranean diet (MD) was $33 \pm 5$ for all participants $(p=0.876)$ and was inversely associated with GDS score (Pearson's $\mathrm{r}=-0.109, p<0.001)$. Normal GDS score was observed in $67 \%$ of participants $(\mathrm{F}=61 \% ; \mathrm{M}=73 \%$ ), while $5 \%$ were severe $(\mathrm{F}=7 \% ; \mathrm{M}=3 \%)(p<0.001)$. Low-density lipoprotein cholesterol (LDL-c) was significantly different $(p=0.008)$ in severe $(106 \pm 44.8 \mathrm{mg} / \mathrm{dL})$ compared to mild depression $(129 \pm 40.3$ $\mathrm{mg} / \mathrm{dL})$ and normal individuals $(123 \pm 41.6 \mathrm{mg} / \mathrm{dL})$. Multinomial logistic regression analysis revealed that higher levels of LDL-c were independently related to increased likelihood of mild symptoms of depression compared to severe symptoms (Odd's ratio $(\mathrm{OR})=1.01,95 \%$ confidence interval $(\mathrm{CI})=$ $1.00-1.02, p=0.04)$ following adjustment for age, MedDietScore, body mass index and sex. In the same multi-adjusted model, higher MedDietScore independently increased the odds of a normal GDS score $(\mathrm{OR}=1.07,95 \% \mathrm{CI}=1.01-1.13, p=0.021)$ compared to severe, independent of LDL-c. In older adults, high diet quality, determined by the MedDietScore is associated with lower symptoms of depression, and low LDL-c levels increase risk of severe depressive symptoms.

\subsection{Consumption of Long Chain Omega 3 Polyunsaturated Fatty Acids and Contribution of Diet Sources and Supplements in Middle Aged to Older Adults}

\section{E. K. Tregoweth ${ }^{1}$, A. M. Coates ${ }^{1}$, D. Greaves ${ }^{1,2}$ and A. E. Smith ${ }^{12}$}

1 Alliance for Research in Exercise, Nutrition and Activity (ARENA), Sansom Institute for Health Research, School of Health Sciences, University of South Australia, Adelaide, SA, Australia

2 Cognitive Ageing and Impairment Neurosciences (CAIN), School of Psychology and Social Work, University of South Australia, Adelaide, SA, Australia

The Australian suggested dietary target (SDT) for long chain omega-3 polyunsaturated fatty acids (LCn-3PUFA) to reduce the risk of chronic disease is $430 \mathrm{mg} / \mathrm{day}$ for women and $610 \mathrm{mg} /$ day for men. We examined the dietary patterns of middle age to older adults ( $\mathrm{n}=90$, between $50-80$ years, 37 males) and evaluated LC $n$-3PUFA consumption contribution from dietary sources and supplements. Cardiovascular disease (CVD) risk was calculated (determined by the presence of diagnosed hypertension, diabetes and hyperlipidaemia) and additionally participants were classified into three age categories: 50-59 (55.23 \pm 2.88 years), 60-69 (66.65 \pm 1.91 years) and 70-80 (74.59 \pm 2.95 years). Consumption of foods and supplements containing LCn-3PUFA was assessed using a validated self-report 41-item food frequency questionnaire and total amount of LCn-3PUFA 
compared with sex-specific SDT guidelines. In total, $60.3 \%$ of females met SDT guidelines compared with $40.5 \%$ of males $(p=0.065)$. From total consumption, LC $n$-3PUFA was predominately sourced from fish (males $48.2 \%$, females $47.9 \%$ ), meat/eggs (males $34.5 \%$, females $29.9 \%$ ) and supplements (males $11.76 \%$, females $21.71 \%$ ). The proportion of females (0.396) supplementing LCn-3PUFA was significantly $(p=0.038)$ higher than males $(0.297)$. The proportion of females in each age category consuming LC $n$-3PUFA supplements increased $(p=0.0076$, proportions: $50-59=0.125,60-69=0.400$, $70-80=0.647)$ and similar increases in supplement usage were evident in people with diagnosed CVD risk across all age categories ( $p=0.008$, proportions: 50-59 $=0,60-69=0.368,70-80=0.625)$. Increased supplementation for both females and people with increased CVD risk is potentially attributed to effective public health messages and a heightened awareness of health with age.

\subsection{Nutrition Status and Cognitive Ability among the Elderly in Two Nursing Homes of China}

\section{P. Song, Q. Man, L. Li, S. Jia and J. Zhang}

Affiliation not provided

To analyze the nutrition status and cognitive abilities of the elderly living in two nursing homes of China. All of the elderly were face-to-face investigated by a questionnaire on general information, health and MNA. ADL and MoCA Scale data were collected. Height and weight were measured, finger blood hemoglobin was tested. In total, 134 participants ( 55 male and 79 female) with mean age 81.7 y were interviewed. More than $96.5 \%$ suffered from more than one disease; the top five diseases were hypertension, skeletal diseases, CVD, digestive diseases and metabolic diseases, which accounted for $61.7 \%, 45.0 \%, 43.3 \%, 35.0 \%$ and $26.7 \%$, respectively. The percentage of impaired vision, hearing loss and impaired masticatory ability was $53.3 \%, 71.7 \%$ and $41.7 \%$, respectively. MNA results showed that $11.7 \%$ of them were well nutritioned, $68.3 \%$ represented a potential malnutrition risk and $20.0 \%$ were malnutritioned. ADL results illustrated that $28.3 \%$ of participants were disabled: $58.9 \%$ were mildly disabled, $23.6 \%$ moderately and $17.6 \%$ severely. Participants with BMI $<18.5 \mathrm{~kg} / \mathrm{m}^{2}$ accounted for $36.6 \%$, anemia accounted for $56.7 \%$ and declined cognitive abilities accounted for $48.1 \%$ of the total. The correlation coefficient between cognitive score and staple foods, milk and products, beans, and vegetables was $0.97,0.90,0.94$ and 0.97 respectively. We concluded that the nutrition status and cognitive abilities in nursing homes were severe and more attention should be paid to nutrition improvement among the elderly living in nursing homes.

\subsection{Musculoskeletal Health in Postmenopausal Malaysian Malay Women}

\section{S. R. Mitra and N. Z. Abidin}

School of Biosciences, Faculty of Science, University of Nottingham Malaysia Campus

Ageing is accompanied with loss of muscle mass and decreased bone mineral. There is a need for screening and appropriate intervention for healthy ageing. The objective of this study was to assess musculoskeletal health in postmenopausal women to screen for risk of sarcopenia and osteoporosis in the study participants. Postmenopausal Malaysian Malay women (56.9 \pm 6.0 years, $\mathrm{n}$ $=26$ ) were recruited. Bone health was assessed by calcaneal quantitative ultrasound (SAHARA, Hologic Inc., Bedford, MA, USA); Hand grip strength was measured using North Coast Hydraulic Hand Dynamometer. Body composition was assessed by bio-electric impedance (InBody 230, Biospace Co. Ltd., Korea). Physical performance was determined using modified Short Physical Performance Battery test. Osteopenia/osteoporosis was defined by WHO standards. (T-scores $<-1$ and $\leq-2.5$ respectively). Higher numbers of the participants $(92.3 \%)$ were obese (percent body fat 43.8 $\pm 7.1 \%$ ). The mean BUA (Bone Ultrasound Attenuation) was $67.9 \pm 13.5 \mathrm{~dB} / \mathrm{MHz}$, comparable with age matched African-American and Caucasian-American women. Average grip strength was 19.3 $\pm 6.4 \mathrm{~kg}$, higher than the cut-off defining the parameter as criteria for sarcopenia (18 kg). AppMMI $\left(\mathrm{kg} / \mathrm{m}^{2}\right)$ (appendicular muscle mass index) was $6.3 \pm 0.7$ just above the cutoff $(>5.7)$. Gait speed was $1.0 \pm 0.2 \mathrm{~m} / \mathrm{s}$ and the score for 'one-leg stance' (right) was $24.6 \mathrm{~s} \pm 7.3$; both above the cut off for 
sarcopenia as defined by Asian Working Group for Sarcopenia (AWGS). However, the participants demonstrated significantly lower scores for 'sit to stand test' (30 s) 9 times $\pm 3.0 p<0.001$ (AWGS $>20$ times). Reduction in body fat is necessary to prevent fat infiltration in muscle and bone marrow to prevent osteosarcopenic obesity, as the participants age.

3.86. Are Baby Boomers Presenting New Dietary Challenges?

O. Farrer, A. Yaxley, K. Walton and M. Miller

College of Nursing and Health Sciences, Flinders University, Adelaide 5042, Australia

Obesity and ageing are catalysts for type 2 diabetes diagnosis, which are at epidemic proportions globally. This presents challenges to health and aged care systems. Current dietary practices in residential aged care (RAC) are primarily aimed at managing malnutrition in older adults, and most facilities have moved away from therapeutic diets for chronic conditions such as diabetes due to concerns of their restrictive nature. However, our emerging ageing population are more likely to be overweight and at least a third of older adults are likely to have diabetes. In an audit of almost 300 residents across 13 RAC sites, findings indicated that $80 \%$ of the older adults with diabetes were within either a healthy or overweight/obese BMI range (average BMI $29-31 \mathrm{~kg} / \mathrm{m}^{2}$ ) at the time of auditing. On further examination of resident demographics, the 'young old' with diabetes (65-74 yrs) were more likely to present to RAC with obesity and comorbidities, and maintain their weight over a typical admission of 24 months, unlike their predecessors ( $>85 \mathrm{yrs})$ who were more likely to lose weight over the same period. In addition, for those residents still receiving a therapeutic diabetic diet, statistical testing suggests that residents are at no more risk of malnutrition than residents on a regular diet code. As such, this may present a need to consider current RAC practices for this cohort who may not be as at risk of malnutrition as the literature would suggest.

\subsection{Systematic Review of Long-Term RCT's of B-Vitamin Supplementation to Improve Cognition in Adults} over 65 Years of Age

N. D'Cunha, E. Georgousopoulou, L. Dadigamuwage, J. Kellett, D. Panagiotakos, A. McKune, D. Mellor and N. Naumovski

Australian National University, Canberra 0200, Australia.

B-vitamin supplementation has been proposed as an approach to mitigate cognitive decline in older adults due to low B-vitamin blood concentrations being associated with mild cognitive impairment (MCI) and Alzheimer's disease. The aim of this systematic review was to evaluate current evidence surrounding B-vitamin supplementation and cognition in double-blinded, randomised controlled trials (RCT) (duration: $\geq 1$ yr; participants: $n=\geq 100$; age (mean): $\geq 65 \mathrm{yr}$ ). Following PRISMA guidelines, PUBMED, Scopus, CINAHL and Web of Science were searched, and nine studies identified; published between 15 June 2006 and 14 June 2016. The included studies used various formulations of vitamin B12 (all studies), folic acid (eight studies) and vitamin B6 (five studies). Only four of the included studies identified benefits to cognition in older adults. One study, in individuals with MCI $(\mathrm{n}=266$; duration $=2 \mathrm{yr})$, reported benefits on executive function $(p=0.015)$ and improvements in Mini-Mental State Examination (MMSE) among participants with baseline homocysteine $(\mathrm{Hcy})>11.3 \mathrm{~mol} / \mathrm{L}(p<0.001)$. In the same cohort, a second study found several cognitive benefits of B-vitamins that depended on high baseline plasma omega-3 fatty acid levels. Another study $(\mathrm{n}=900$; duration $=2 \mathrm{yr})$ reported improved performance in immediate $(p=0.046)$ and delayed recall $(p=0.013)$, while the fourth study $(\mathrm{n}=2919$; duration $=2 \mathrm{y})$ reported a slower rate of cognitive decline in the MMSE $(p=0.05)$. The results revealed that two years of B-vitamin supplementation may have benefits on cognition in older adults, but this may also depend on other factors including, Hcy and omega-3 fatty acid status. Additional long-term trials of B-vitamins 
investigating these interactions with other blood biomarkers and genetic polymorphisms are required.

\subsection{Relationship of B-Vitamin Biomarkers and Dietary Intake with APOE4 in Alzheimer's Disease}

N. D'Cunha, E. Georgousopoulou, L. Boyd, J. Sturm, M. Lucock, A. McKune, J. Thomas, D. Mellor, P. Roach and N. Naumovski

Australian National University, Canberra 0200, Australia

Reducing elevated plasma homocysteine (Hcy) with B-vitamin supplementation offers a potential preventative measure for Alzheimer's disease (AD). However, the association between Apolipoprotein E-e4 (APOE4), the strongest genetic risk factor for AD, biomarkers of B-vitamin status (Serum B12, Serum Folate [SF], Hcy, Cysteine [Cys] and Cysteinyl-Glycine) and dietary Bvitamin intakes are not yet fully understood. In this retrospective case-controlled study, in elderly age-matched Australians ( $\mathrm{n}=126)$, the APOE4 phenotype was determined by ELISA for 63 AD cases $($ female $=35$, male $=28 ;$ age $=77.1 \pm 5.3 \mathrm{yr}$ ) and 63 healthy controls $($ female $=38$, male $=25 ;$ age $=76.9$ $\pm 4.7 \mathrm{yr}$ ). The plasma concentrations of the B-vitamin biomarkers were determined using highpressure liquid chromatography, and the dietary B-vitamin intakes were determined by food frequency questionnaire and the FoodWorks software. APOE4 was found in 43.7\% (55/126) of all participants, with $29.4 \%$ (37/126) of all participants possessing both AD and the APOE4 phenotype. Initial analysis showed that there was no evidence of an association between the B-vitamin biomarkers and AD relative to APOE4 phenotype (all, $p>0.05$ ). However, subsequent analysis of covariates using receiver operating characteristic curves revealed an increased likelihood of AD when Hcy levels were $>11.0 \mathrm{umol} / \mathrm{L}(p=0.012)$, Cys levels were $<255 \mathrm{umol} / \mathrm{L}$ and SF was $<22.0 \mathrm{nmol} / \mathrm{L}(p=$ 0.003; in males only). In females only, dietary intake of B-vitamins (total folate $<336 \mathrm{ug} /$ day [ $p=0.001$ ], natural folate $<270 \mathrm{ug} /$ day $[p=0.011]$ and riboflavin $<1.12 \mathrm{mg} /$ day $[p=0.028]$ ) was associated with an increased likelihood of AD. These results suggest that Hcy, Cys, and SF are useful biomarkers for $\mathrm{AD}$, irrespective of APOE4 phenotype. However, the gender differences related to dietary B-vitamin intake and $\mathrm{AD}$ require further investigation.

\subsection{Nutrition in Older Adults at Risk of Cardiovascular Disease: Association with Quality of Life}

\section{T. Govindaraju ${ }^{1}$, T. A. McCaffrey ${ }^{2}$, C. M. Reid ${ }^{1,3}$ and A. J. Owen ${ }^{1}$ \\ 1 School of Public Health \& Preventive Medicine, Monash University, Victoria 3000, Australia \\ 2 Department of Nutrition and Dietetics, Monash University, Victoria 3000, Australia \\ 3 School of Public Health, Curtin University, Bentley, WA 6102, Australia}

Both nutrition and quality of life are key determinants of healthy ageing. While studies have consistently shown a relationship between nutrition and health across age-groups, very few studies have looked at the association between nutrition and quality of life (QoL) in older adults. In 460 participants aged 60 years and above, with known cardiovascular risk factors, dietary intake was assessed using weighed 4 day food records and QoL using the SF36 questionnaire. Correlations were analysed across the eight domains of QoL and by physical and mental component summaries. Total energy intake was significantly positively correlated with the physical functioning QoL domain, $(p=$ $\left.0.005, r_{s}=0.135\right)$. However, there were significant negative correlations between percent energy derived from carbohydrates and two of the physical domains physical functioning ( $p=0.005, \mathrm{rs}=$ $-0.137)$ and role limitations due to physical health $\left(p=0.031, \mathrm{r}_{\mathrm{s}}=-0.105\right)$. There was also a significant negative correlation between percent energy from carbohydrates and the overall physical component summary (PCS) $\left(p=0.033, \mathrm{r}_{\mathrm{s}}=-0.103\right)$. No significant correlations were observed between energy or macronutrients and mental component summary (MCS) of the QoL scale.

While total energy intake is positively associated with QoL in older adults, the source of dietary energy may be a key factor, with this study finding a significant negative association between dietary 
carbohydrate and the physical domains of QoL. These findings highlight the need for further research examining the role of diet and nutrition in quality of life in older age.

\subsection{Australian Adults: Drink Choice Varies by Age}

G. Hendrie, D. Baird and M. D. Riley

Commonwealth Scientific and Industrial Research Organisation (CSIRO) Health and Biosecurity, Adelaide 5000, Australia

Consuming beverages is common dietary behavior for Australian adults, and intake of soft drinks and other manufactured sugar sweetened beverages in particular is implicated to contribute to excess energy intake and obesity. However, beverage intake as a category is rarely examined because of technical reasons of separating fluid foods consumed as a drink from fluid foods used in mixed dishes, and because stakeholder interest is often in a specific set of beverages. This study aimed to describe all beverage intake and its contribution to nutrient intake for Australian adults as assessed in the 2011-2012 National Nutrition Survey: the 24-h dietary recall records of 9361 Australian adults. Data was weighted for age, gender, area of residence and day of recall. Beverages contributed $16 \%$ to the total energy intake for Australians 19 years of age and over, with alcoholic beverages making the highest contribution (5.3\%) followed by coffee (3.2\%). Water was consumed in the largest mean daily amount $(1102 \mathrm{~g})$ and by the highest percentage $(85 \%)$ on the day of the survey. Aside from water intake, the top two ranked beverages by mean intake were soft drinks and alcoholic drinks for 19-30 year olds, coffee and alcoholic drinks for 31-50 year olds, tea and coffee for 51-70 year olds and tea and coffee for people aged 71 years and above. Most Australian adults drink significant amounts of water; but tea, coffee and alcoholic drinks are important contributors to beverage intake.

\subsection{Five Food Group Knowledge in Adults Aged 70 Years and over in Adelaide}

H. Morris 1, Y. W. Choo 1,2, A. Cramond 1,2, M. De Sciscio 1,2, T. C. Lee ${ }^{1,2}$, K. Lush 1,2, W. Y. Kong 1,2, A. Mavrogiannis 1,2, P. Schwarz 1,2, T. Wang 1,2, Y. Y. Wu 1,2, S. S. Yeoh 1,2, N. C. Y. Yeung 1,2, S. Cameron ${ }^{3}$ and S. J. Zhou ${ }^{1}$

1 School of Agriculture, Food and Wine, University of Adelaide, Adelaide, SA 5005, Australia

2 Food and Nutrition Science Students, University of Adelaide, Adelaide, SA 5005, Australia

3 Wellness Centre, Royal Adelaide Hospital, Adelaide, SA 5000, Australia

The Five Food Group (FFG) system is an important component of the Australian Eat for Health Program, which is designed to assist healthy Australians plan nutritionally balanced meals and snacks. Adults aged 70 years and over have a higher risk of inadequate nutritional intake but little is known about their awareness of the FFG tool. This survey aimed to assess awareness and ability to identify FFG in adults aged 70 years and over. Participants $(n=170)$ were recruited from the front entrance of the Royal Adelaide Hospital. The survey questionnaire included questions on knowledge of the FFG and 10 food photos of popular, commonly eaten healthy meals and snacks relevant to the target age group, for the identification of the FFG. Individual foods were labelled in each photo to ensure participants recognised the food. Sixty-three percent reported that they were aware of the FFG, but only $6 \%$ were able to name all of the five food groups correctly. The percentage able to name each food group ranged from $19 \%$ for the dairy group to $44 \%$ for the vegetable group. More than $50 \%$ $(52-98 \%)$ of participants were able to categorise all foods in each of the seven photos that did not contain nuts or legumes into the appropriate food group compared with $\leq 35 \%$ (14-35\%) in the three photos contained nuts and legumes. Further research to identify enablers and barriers to improve FFG literacy in this age group may improve the utility of the FFGs as a menu planning tool.

\subsection{Prevalence and Factors Associated with Geriatric Poor Nutrition Status in China: A Multi-Central, Cross-Sectional Study}

J. Q. Sun ${ }^{1}$, H. L. Zhu ${ }^{2}$, W. Chen ${ }^{3}$, B. Q. Mo ${ }^{4}$, C. Y. Huang 5 , D. F. Xu ${ }^{1}$, Y. Q. Chen ${ }^{1}$, H. Xie ${ }^{1}$, Q. Ren ${ }^{1}$, P. Fu ${ }^{6}$ and J. Zhang ${ }^{6}$ 
1 Clinical Nutrition Centre, Huadong Hospital, Fudan University, Shanghai, China

2 Department of Nutrition, School of Public Health Sun Yat-sen University, China

PN\&EN Service, Peking Union Medical College Hospital, China

School of Public Health Nanjing Medical University, China

West China School of Public Health Sichuan University, China

6 National Institution for Nutrition and Food Safety, Chinese Center for Disease Control and Prevention, China

Malnutrition is highly prevalent among the elderly and is associated with decreased functional status and increased mortality. To estimate the prevalence of geriatric poor nutrition status (PNS) and investigate the associated factors, 5979 participants (65 years old and more) who attended clinics were enrolled. Demographic data, anthropometric data, blood hemoglobin and plasma albumin levels, Activities of Daily Life (ADL) scale and Mini Nutritional Assessment-Short Form (MNA-SF) scale were collected. According to MNA-SF score, $16.13 \%$ participants suffered overt malnutrition and $37.61 \%$ at risk of malnutrition. Compared with well-nutritional patients, malnutrition patients had lower BMI $(p<0.0001)$, calf circumference (CC, $p<0.0001)$, mid-arm circumference (MAC, $p<$ $0.0001)$, hand grip strength $(p<0.0001)$, albumin level $(p<0.0001)$, hemoglobin level $(p<0.05)$ and ADL scale score $(p<0.0001)$. Meantime, BMI lower than $22.0 \mathrm{~kg} / \mathrm{m}^{2},(O R=7.186,95 \% \mathrm{CI}: 6.304-8.191)$, age more than 80 years old $(O R=1.984,95 \% \mathrm{CI}: 1.770-2.225)$, CC less than $31 \mathrm{~cm}(O R=6.829,95 \% \mathrm{CI}$ : 5.909-7.893), MAC less than $25.3 \mathrm{~cm}$ for male or $23.2 \mathrm{~cm}$ for female $(O R=4.612,95 \% \mathrm{CI}: 4.001-5.316)$, hand grip strength less than $26 \mathrm{~kg}$ for male or $18 \mathrm{~kg}$ for female (OR $=2.527,95 \%$ CI: 2.172-2.940), diagnosed anemia $(O R=1.865,95 \% C I$ : $1.563-2.225)$, diagnosed hypoalbuminemia $(O R=2.131,95 \%$ CI: $1.708-2.658)$, inferior masticatory function $(O R=3.139,95 \%$ CI: $2.808-3.509)$, sick dentition status $(O R=1.384,95 \%$ CI: $1.220-1.572)$ were associated with PNS. The prevalence of PNS in geriatric patients was relatively high in medical institutions in China, especially in general hospitals and nursing homes. Lower BMI, aging, decreased CC, MAC, hand grip strength, existed anemia or hypoalbuminemia, impaired masticatory function and poor dentition status can increase the risk of PNS.

\subsection{The MIND Diet but Not the Mediterranean Diet Is Associated with Reduced Incidence of 12-Year Cognitive Impairment in Older Australians}

H. Diane, R. Eramudugolla and K. Anstey

College of Health and Medicine, Australian National University, Canberra 0200, Australia

Evaluating the role of dietary factors in reducing dementia risk is challenging due to populationspecific characteristics. We aimed to test if the newly developed MIND diet was protective against cognitive decline in an Australian setting, and compare it for the first time with both the traditional Greek and the more common 9-point Mediterranean Diet. Participants $(n=1228)$ aged $72-76(50.7 \%$ female) were drawn from the PATH Study, a longitudinal study of health and ageing based in Canberra, ACT. MIND diet, and two alternate Mediterranean diet scores were calculated from baseline responses to the CSIRO-FFQ. At 12-year follow-up, participants were screened for cognitive decline. In adjusted logistic regression models, the MIND diet but not the Mediterranean Diet was associated with reduced odds of 12-year incident cognitive impairment $(\mathrm{OR}=0.81,95 \% \mathrm{CI} 0.68,0.96$; $\mathrm{B}=-0.20, p=0.019)$. APOE- $\varepsilon 4$ made the only other significant contribution ( $\mathrm{OR}=2.28,95 \% \mathrm{CI} 1.46$, $3.48 ; \mathrm{B}=0.82, p<.001)$. The interaction term for MINDxAPOE- $\varepsilon 4$ was non-significant. When the MIND score was split into tertiles, those in the highest tertile of MIND diet had a $51 \%$ reduction in the odds of impairment ( $\mathrm{OR}=0.49,95 \% \mathrm{CI} 0.260 .93 ; \mathrm{B}=-0.70, p=0.029)$.

Our study provides preliminary evidence that the cognitively protective effects of the MIND diet generalise to a setting outside the USA. The MIND diet is not as culturally or sample-specific as the Mediterranean diet and may be useful to inform dietary guidelines for cognitive health. Further research is required comparing the MIND and the Mediterranean diets across diverse populations and locations. 
3.94. Dietary Profile of Older Australians Who Met the Recommended Added Sugar Intake of Less Than 5\% of Energy: Findings from the Blue Mountains Eye Study Cohort

H. Moshtaghian ${ }^{1}$, K. E. Charlton 1,2, J. C. Y. Louie ${ }^{3}$, C. Y. Probst ${ }^{1,2}$, P. Mitchell ${ }^{4}$ and V. M Flood 5,6

1 School of Medicine, University of Wollongong, Wollongong, Australia

2 Illawarra Health and Medical Research Institute, Wollongong, Australia

3 School of Biological Sciences, The University of Hong Kong, Pokfulam, Hong Kong, China

4 Center for Vision Research, Westmead Institute, University of Sydney, Sydney, Australia

5 Faculty of Health Sciences, University of Sydney, Sydney, Australia

6 Westmead Hospital, Sydney, Australia

Previous studies reported inconsistent findings regarding dietary intakes of added sugar (AS) consumers. This study aims to describe the dietary profile of an older Australian population who met the recommended energy intake from AS (EAS\%) of less than $5 \%$. Subjects were participants of the Blue Mountains Eye Study (aged $\geq 49$ years) who had dietary data at both baseline (1992-1994) and the 15-year follow-up $(\mathrm{n}=864)$. Dietary data were collected using a 145-item food frequency questionnaire. AS content of foods was estimated using a 10-step systematic method. Participants were classified into three categories based on EAS\% guidelines $(<5 \%, 5-10 \%$ and $>10 \%)$. A generalized linear model was used to evaluate the difference in macronutrients and food groups intakes between EAS\% categories in the multivariate adjusted model. A cross-tabulation was used to assess the proportion of participants remaining in the same category or shifting to other categories between baseline and follow-up. At both baseline and the follow-up, participants in the lowest EAS\% category $(<5 \%)$ reported higher alcohol, protein, fiber, fruit, vegetable and fish intakes compared to others $(p<0.05)$. Furthermore, they had lower intakes of food sources of AS (e.g., sugar, sweet spreads and confectionary) compared to others $(p<0.05)$. More than $60 \%$ of participants with baseline EAS\% of $<5 \%$ were classified into the higher categories during follow-up. In this study, while older participants with low AS intake had higher intakes of nutrient-dense foods, this was accompanied by higher alcohol consumption. The low long-term adherence rate to strict EAS \% recommendations warrants further investigations in other populations.

\subsection{Older Australians Are Eating More Protein but a Poorer Quality Diet-Secondary Analysis of the} National Surveys in 1995 and 2011/12

\section{F. O'Leary, Z. Sui, H. Cheng, A. Rangan and V. Hirani}

Discipline of Dietetics, School of Life and Environmental Science and the Charles Perkins Centre, The University of Sydney, Australia

Diet is important in healthy ageing. Protein is a key nutrient essential for physical function, immunity, maintenance of quality of life and the ability to live independently. This study examined protein intakes, dietary sources, and diet quality at two time points among older Australians. Analyses included data from adults aged $\geq 65$ years participating in the 1995 National Nutrition survey ( $n=1960$ ) and 2011/12 National Nutrition and Physical Activity Survey (2103) that included measures of dietary intakes, demographic, anthropometric and health information. Linear trend analysis of protein intake adjusted by demographic, anthropometric and dietary intakes were conducted. Protein adequacy was assessed using the Australian Estimated Average Requirements (EAR). Participants reported higher total protein intake in 2011/12 than in 1995 (79.4 vs. 73.4 g, $p<$ 0.001). Protein intake by body weight (1.0 g/kg in 1995 and 2011/12) and inability to meet the EAR ( $22.7 \%$ in 1995 vs. $21.0 \%$ 2010/11) were similar at both time points, due to increasing body weight in 2011/12. In both surveys, higher protein intake was associated with greater consumption of vegetables, dairy products, meat and alternatives and lower consumption of discretionary foods and beverages. Unprocessed lean red meat $(<10 \%$ fat) was the main protein source in both surveys. Diet quality was lower in 2011/12, with reductions in vegetables, wholegrains and dairy foods and increases in snacking and discretionary beverages. Specific dietary advice for older Australians is needed to promote healthy food choices with adequate protein content that may benefit disease prevention and maintenance of quality of life. 
3.96. Association between Sarcopenia and Metabolic Syndrome in Middle and Aged Non-Obese Adults: A Systematic Review and Meta-Analysis

H. Zhang, S. Lin, F. Zhong and A. Ma *

Department of Public Health, Medical College of Qingdao University, Qingdao, China

* Corresponding Author

Sarcopenia, as an age-related loss of muscle mass and fuction, is responsible for a great global burden of health problems. However, the associations between sarcopenia and metabolic syndrome (MetS) in non-obese middle and aged adults remain controversial and no quantitative meta-analysis exists. Thus, this meta-analysis aimed to evaluate the overall prevalence of MetS and the correlations between sarcopenia and MetS in non-obese middle and aged adults. We performed a systematic search strategy using PUBMED, EMBASE and Web Of Science database for relevant observational studies investigating sarcopenia and MetS until 11 May 2017. Participants' emographics, denition of sarcopenia and MetS were retrieved. The polled prevalence of MetS and odds ratios with 95\% confidence intervals (CI), as well as subgroup analyses were calculated using a random effects model. Twelve articles with a total of 35,581 participants were included. The overall prevalence of MetS was $36.45 \%$ (95\% CI, $28.28-45.48 \%$ ) and $22.81 \%$ (95\% CI, 17.97-28.51\%) in non-obese middle and aged adults with or without sarcopenia, respectively. Our analysis demonstrated a positive association between sarcopenia and MetS (OR $=2.01,95 \% \mathrm{CI}, 1.63-2.47)$. The subgroup analysis showed that larger sample size and using Dual-energy $\mathrm{X}$-ray absorptiometry for measuring body composition can enhance the relationship. Our study revealed a higher proportion of MetS in middle and aged nonobese people with sarcopenia. Moreover, sarcopenia was positively associated with MetS in this population. Futher large-scale prospective cohort studies are needed to investigate the causality between sarcopenia and MetS.

Acknowledgments: This work was supported by the National Natural Science fund (No. 81373000).

\subsection{DIETS of People in the Pacific Islands}

J. Santos ${ }^{1}$, B. McKenzie ${ }^{1}$, J. Wate ${ }^{2}$, A. Pillai ${ }^{2}$, C. Johnson ${ }^{1}$, A. M. Thow ${ }^{3}$, C. Bell ${ }^{4}$, W. Snowdon ${ }^{4}$ and J. Webster ${ }^{1}$

1 The George Institute for Global Health, University of New South Wales, Sydney, Australia

2 Fiji National University, Nasinu, Fij

3 Menzies Institute, Sydney, Australia

4 Global Obesity Centre, Deakin University, Geelong, Australia

Pacific Islanders are experiencing a high burden of diet-related non-communicable disease (NCD), yet there has been no comprehensive review of information on diets in the region. This study aimed to identify and summarize available literature on Pacific Island diets, with a specific focus on salt, fat and sugar intake. Electronic databases (Pubmed, Scopus, ScienceDirect, GlobalHealth) were systematically searched from 1990 to April 2017 using relevant terms. Additional sources from grey literature and key stakeholders were also included. Eligibility of the studies was assessed by two authors and quality evaluated using a modified tool for assessing dietary intake studies. Sixty-four studies (38 peer-reviewed and 26 from other sources) met the inclusion criteria. Most studies included information on fat intake ( $n=50$ studies), while information on sugar and salt intake was less common (34 and 20 studies respectively). Whilst dietary assessment methods and the type of data reported varied considerably, a common finding was that levels of fat, sugar and salt were increasing-likely due to the shift in dietary patterns from traditional to imported foods. The study quality also varied - peer-reviewed studies used more robust dietary assessment tools but lacked representativeness, while the reports from national surveys were more representative but often employed indirect methods to quantify dietary intake. The available data suggests that Pacific Islanders are consuming high and increasing levels of fat, sugar and salt. However, more comprehensive national dietary surveys are required to better understand the changing diets of people living in Pacific Islands and inform effective policy interventions. 


\title{
3.98. Proposal of New Criteria for Low Skeletal Muscle Mass in Chinese Adults: A Cross-Sectional Observational Study
}

Wei Chen 1, Xiaohui Feng 2, Hailong Li 1, Junren Kang 1, Xiaodong Shi 1, Wenguang Sun 2, Aiqin Ma 2, Tingting Lv 2, Haili Liu 2, Yan Guan 3, Qin Du 3, Menaka Yalawar ${ }^{4}$ and Sheng Ge 2,*

1 Peking Union Medical College Hospital, Beijing, China

2 Sixth People's Hospital Affiliated Shanghai Jiaotong University, Shanghai, China

3 Abbott Nutrition Research and Development, Shanghai, China

4 Cognizant Technology Solutions, Bengaluru, India

* Correspondence: gesheng607@126.com

(1) Background: Low skeletal muscle mass (LSMM) is defined as age-related loss of muscle mass and is one of the symptoms of sarcopenia. Measurement methods and criteria for LSMM have been established in Europe and Asia. However, there is less evidence of muscle mass in mainland Chinese. In order to develop cut-off points to determine LSMM, we examined muscle mass using the bioelectrical impedance analysis (BIA) technique and validated results against dual energy $\mathrm{x}$-ray absorptiometry (DEXA) measurements in healthy young Chinese adults; (2) Methods: A total of 423 healthy adults aged 25-34 years old were recruited from Beijing and Shanghai, and their anthropometrics, body compositions, hand grip strength, and gait speed were assessed; (3) Results: In the overall population, total skeletal muscle mass (TSMM) was $21.74 \pm 5.09 \mathrm{~kg}$ measured by DEXA and $21.74 \pm 5.09 \mathrm{~kg}$ by BIA, and skeletal muscle mass index (SMI) was $7.74 \pm 1.26 \mathrm{~kg} / \mathrm{m}^{2}$ measured by DEXA and $8.03 \pm 1.15 \mathrm{~kg} / \mathrm{m}^{2}$ by BIA. Hand grip strength was $35.1 \pm 10.0 \mathrm{~kg}$ and gait speed was $1.55 \pm$ $0.28 \mathrm{~m} / \mathrm{s}$. TSMM was correlated with hand grip strength, gait speed and calf circumference in both genders regardless of measurement methods $(p<0.05)$. We determined that gender-specific cut-off values for the LSMM were $7.83 \mathrm{~kg} / \mathrm{m}^{2}$ for men and $5.97 \mathrm{~kg} / \mathrm{m}^{2}$ for women via BIA. 4) Conclusions: Our findings firstly established normal skeletal muscle mass and muscle function data in Chinese Healthy Urban Youth; this is valuable for establishing specific diagnosis criteria of sarcopenia in the Chinese population.

3.99. Food Allergy Readiness and Anaphylaxis Management in Early Childhood Education and Care (ECEC) Services in Western Australian

\author{
R. Sambell ${ }^{1}$, K. Jacobson ${ }^{1}$, S. Vale ${ }^{2}$ and A. Devine ${ }^{1}$ \\ 1 Edith Cowan University, Joondalup 6027, Australia \\ 2 Australasian Society of Clinical Immunology and Allergy (ASCIA), Balgowlah 2093, Australia
}

Food allergy in young children is rising in Australia (2014: 1 in 57, 2016: 1 in 20). ECEC Services will have increasing numbers of children enrolled with a risk of a severe allergic reaction. Previous research recommends risk minimisation strategies to protect children. The objective of this quantitative study examined the status of food allergy management in ECEC Services in Western Australia (WA) to assess the centres current food allergy readiness. An online questionnaire was emailed to all ECEC services in WA $(n=550)$. In total, 53 centres $(9.6 \%$ response rate) completed the questionnaire. Of these, 51 centres had a policy specifically addressing food allergy. In total, $36 \%$ of services store autoinjectors in locked cabinet. Three services did not require any training for their staff in first aid and/or anaphylaxis. Centres used resources that attracted a fee and only a few (22\%) centres used free resources, despite "cost" and lack of "video resources" being barriers for training staff. This study demonstrated a high level of self-reported confidence and skills, however it revealed gaps in risk minimisation knowledge, especially regarding autoinjectors and available resources. In future, more emphasis on awareness and increased utilisation of existing resources is required for ECEC Services.

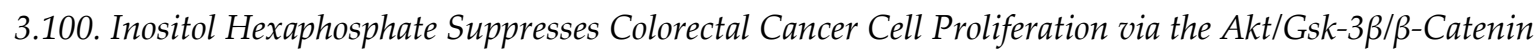
Signaling Cascade in a 1,2-dimethylhydrazine-Induced Rat Model

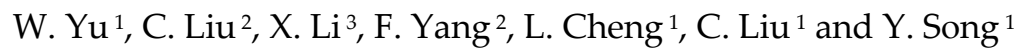


School of Public Health, Qingdao University Medical College, Qingdao, China

School of Nursing, Qingdao University Medical College, Qingdao, China

School of Basic Medicine, Qingdao University Medical College, Qingdao, China

Colorectal cancer (CRC) is common worldwide, and most treatments for CRC have undesirable side effects. Many researchers have demonstrated that inositol hexaphosphate (IP6) has potent anticarcinogenic activity against CRC and no apparent toxicity to normal cells. However, the underlying mechanism is still unclear. In this study, we investigated the anticancer and antiproliferative properties of IP6 in CRC and its possible mechanisms during this chemopreventive process. We examined the expression of genes related to the PI3K/Akt and Wnt pathways at the transcriptional and translational levels in a DMH-induced rat CRC model following IP6 administration. In addition, we also conducted cell proliferation analysis. The results demonstrated that IP6 could inhibit tumors, in terms of tumor incidence, number, weight and volume in DMHinduced rats. Additionally, Akt and C-myc mRNA levels were significantly decreased. IP6 was also shown to downregulate Akt, pAkt, pGSK-3 $\beta$, and C-myc protein expression and upregulate $\mathrm{p} \beta$ catenin protein expression. Furthermore, tumor tissues from IP6-treated rats showed decreased proliferation. In conclusion, the anti-proliferative effect of IP6 may be related to crosstalk between the PI3K/Akt and Wnt pathways, revealing a potential mechanism of CRC inhibition by IP6 in our model.

3.101. Targeting the Endoplasmic Reticulum: Sulforaphane Improved the Abnormal Lipid Metabolism via ire1-xbp1-srebp1 Pathway

S. Tian, J. Ren, P. Lei, L. Qu and Y. Shan

Department of Food Science and Engineering, School of Chemistry and Chemical Engineering, Harbin Institute of Technology, Harbin, China

Sulforaphane (SFN), a photochemical that exists in cruciferous vegetables, has been paid much attention for its potential in the prevention of various types of cancers. However, there is no elaboration about whether SFN presents a preventive effect on the abnormal lipid metabolism and the underlined mechanisms. Human hepatocytes line HHL-5 were exposed to $250 \mu \mathrm{mol} / \mathrm{L}$ FAs (oleic acid: palmitic acid, 1:2) for 5 days to establish the abnormal lipid metabolism model in vitro. Wistar rats were fed on the high fat diet (45\% fat, $35 \%$ protein and $20 \%$ carbohydrate) to mimic the nonalcoholic fat liver disease (NAFLD) in vivo. SFN pre-treatment significantly decreased the intercellular levels of TG and TC induced by FAs. The swelling endoplasmic reticulum (ER) and the increased perimeter of the ER caused by FAs was obviously alleviated by SFN. SFN decreased the expressions of lipogenic enzymes such as ACC, SCD1 and FAS1 both in the transcriptional and translational levels through blocking the ER stress targeting the IRE1-XBP1-SREBP1. Blocking the ER stress, the accumulation of lipids was largely relieved which is accompanied by the downregulation of XBP1mRNA. SFN prevented the abnormal accumulation of lipids. The underlined mechanism was involved in blocking the ER stress through the IRE1-XBP1-SREBP1 pathway.

\subsection{Protective Effects of Sulforaphane on the Alcohol-Induced Toxicity and Endoplasmic Reticulum Stress in C57BL/6 Mice}

Peng Lei, Wei Zhao, Xiaodong Liu and Yujuan Shan

Department of Food Science and Engineering, School of Chemistry and Chemical Engineering, Harbin Institute of Technology, Harbin, China

Department of Food Science and Engineering, School of Chemistry and Chemical Engineering, Harbin Institute of Technology, Harbin 150090, China

It is known that alcoholic liver disease (ALD) caused by acute and chronic exposure to alcohol is one of the chronic liver diseases with high mortality. Sulforaphane (SFN), rich in various cruciferous vegetables, has been widely studied as a chemo-preventive agent. In this study, we focused on the protective effects of SFN on alcohol-induced toxicity and mechanisms. Male C57BL/6 
mice were orally administrated with SFN $(0,12,40,80 \mathrm{mg} / \mathrm{kg} . \mathrm{bw})$ for 14 days. At the 13th day, mice were challenged with alcohol $(5 \mathrm{~g} / \mathrm{kg} . \mathrm{bw})$ every $12 \mathrm{~h}$ three times. SFN markedly reversed the alcoholinduced decrease of antioxidant capacity through enhancing GSH, GSH-Px, GST redox system. Furthermore, the protective actions are related with activating Nrf2 and inactivating NF- $\kappa$ B. SFN attenuated the triglyceride (TG) and cholesterol (CHOL) contents which is possibly mediated by down-regulating sterol regulatory element-binding protein-1 (SREBP-1c). In addition, SFN weakened the stimulation of the chaperone GRP78, and its downstream sensory receptor ATF-6, triggered by acute alcohol intake. The current findings indicated that SFN weakened the liver toxicity triggered by alcohol through enhancing the antioxidant capacity and reducing the endoplasmic reticulum stress.

\subsection{Relationship between Urinary Aflatoxin M1 (AFM1) with Food Consumption among Healthy Adults in Hulu Langat District, Selangor}

\section{S. Siti Husna, J. Rosita and S. Mohd Redzwan}

Department of Nutrition and Dietetics, Faculty of Medicine and Health Science, Universiti Putra Malaysia, 43400 Serdang, Selangor, Malaysia

A cross sectional study was conducted to identify the relationship between urinary aflatoxin $\left(\mathrm{AFM}_{1}\right)$ readings with food consumption. A total of 444 urine samples were collected from the district of Hulu Langat and 199 (44.8\%) of them were positive with $\mathrm{AFM}_{1}[$ mean $\pm \mathrm{SD}=1.23 \pm 0.91 \mathrm{ng} / \mathrm{mL}$; range $=0.65$ to $5.34 \mathrm{ng} / \mathrm{mL}$ ]. In total, $18.59 \%(\mathrm{n}=37)$ of these 199 samples had AFM1 concentration that exceeded the limit of detection. Foods that were potentially contaminated with aflatoxin were listed in the Food Frequency Questionnaire (FFQ) and categorized under four categories: (1) cereals and cereal-based products, (2) eggs, (3) nuts and nut-based products and (4) milk and dairy-based products. Of those four food categories, it was found that only milk and dairy-based products had a significant relationship with the $\mathrm{AFM}_{1}$ readings. Respondents with high intake of milk and dairybased products (median $=77.68 \mathrm{~g} /$ day) had significantly high AFM1 readings compared to those with low intake. Thus, high intake of milk and dairy-based products increased AFM1 concentration more favourably than low intake of milk and dairy-based product $(p<0.01)$. The other three food groups (cereals and cereal-based products, eggs, and nuts and nut-based products) did not show any significant findings.

Keywords: Aflatoxin; Aflatoxin M1; Food consumption; Malaysia

\subsection{Effect of Diet on Blood Viscosity in Healthy Humans: A Systematic Review}

\section{H. Naghedi Baghdar and S. Nazari}

Masshad University of Medical Sciences, Mashad, Iran

Increased whole blood viscosity is associated with increased risk of morbidity and mortality of several life-threatening diseases, including cardiovascular and cerebrovascular disease. The effect of diet on human health has been indicated in many studies and a health dietary pattern can reduce the incidence of several chronic diseases. This systematic review aimed to assess the effect of diet on blood viscosity and related parameters such as haematocrit (HCT). MEDLINE, Embase, Scopus, Web of Science, and the Cochrane Central Register of Controlled Trials were searched from inception to 22 November 2016. Some of the main keywords were Blood Viscosity, Haemorheology, Blood Fluidity, Diet, Food, and Nutrition. Only randomized controlled trials were considered. Three randomized controlled trials were included in this systematic review. A different diet was used in the included trials. One study significantly reduced the blood passage time in the control group from $55.4 \pm 3.4$ to $47.6 \pm 2.0 \mathrm{sec}$ (mean $\pm \mathrm{SEM}, p<0.05$ ), while no significant change was observed in the placebo group. Another study has shown significantly increased blood fluidity score (BFS) in a vegetarian group in contrast to the control group after six weeks. In the last study, plasma viscosity was significantly decreased compared to the placebo group with a highly significant difference 
between the two groups $(p=0.0015)$. The results show that the diet used in these trials may reduce blood viscosity in healthy humans. Additional well-designed trials are needed to investigate the efficacy of various diets on blood viscosity.

\subsection{Sweet Taste Perception Influences Sweet Food Liking and Intake}

\section{S. N. Jayasinghe ${ }^{1}$, R. Kruger ${ }^{1}$, D. C. I. Walsh ${ }^{2}$, G. Cao ${ }^{1}$, M. Richter ${ }^{1}$ and B. H. Breier ${ }^{1}$}

1 School of Food and Nutrition, College of Health, Massey University, Auckland, New Zealand

2 Institute for Natural and Mathematical Sciences, College of Sciences, Massey University, Auckland, New Zealand

Background: A range of psychophysical taste measurements are used to characterize an individual's sweet taste perception and to assess links between taste perception and dietary intake. The aims of this study were to investigate the relationship between four psychophysical measurements of sweet taste perception, and to explore which measures of sweet taste perception are linked to sweet food intake.

Methods: Forty-four women aged 20-40 years were recruited for the study. Four measures of sweet taste perception (detection and recognition thresholds, and sweet taste intensity and hedonic liking of suprathreshold concentrations) were assessed using glucose as the tastant. Dietary measurements included a weighed food record, a sweet food-food frequency questionnaire and a sweet beverage liking questionnaire.

Results: We observed significant inter-individual variations in all four sweet taste measurements. Glucose detection and recognition thresholds correlated positively, but showed no correlation with suprathreshold taste measurements or any dietary intake measurement. Sweet taste intensity correlated negatively with total energy, carbohydrate and sugar intakes $(r=-0.4, p<0.01)$, frequency of sweet food intake $(r=-0.3, p=0.03)$ and sweet beverage liking $(r=-0.4, p=0.005)$. Furthermore, sweet hedonic liking correlated positively with total energy and carbohydrate intakes $(r=0.4, p<0.05)$.

Conclusion: This study shows for the first time a clear link between sweet taste intensity and hedonic liking with sweet food liking and intake.

\subsection{Dietary Protein and Amino Acids Profile in Relation to Risk of Dysglycemia: Findings from a Prospective Study}

\section{Zahra Bahadoran, Parvin Mirmiran and Fereidoun Azizi}

Research Institute for Endocrine Sciences, Shahid Beheshti University of Medical Sciences, Tehran, Iran

Objective: Considering the limited data on effects of dietary amino acid intakes on dysglycemia, we assessed the possible association of dietary protein and amino acid patterns with the risk of prediabetes in a prospective population-based study. Methods: Participants without diabetes and prediabetes $(n=1878)$ were recruited from the Tehran Lipid and Glucose Study and were followed a mean of 5.8 years. Dietary protein and amino acid intakes were assessed at baseline (2006-2008); demographic, lifestyle and biochemical variables were evaluated at baseline and follow-up examinations. Pre-diabetes was defined as $5.55 \mathrm{mmol} / \mathrm{L} \leq \mathrm{FPG}<7 \mathrm{mmol} / \mathrm{L}$ and/or 2 -h PCPG $<7.77$ mmol/L according to the American Diabetes Association criteria. Multivariate Cox proportional hazard regression models, adjusted for potential confounders, were used to estimate risk of prediabetes across tertiles of dietary amino acids. Results: Mean age of participants ( $44.9 \%$ men) was 38.3 $\pm 12.7 \mathrm{y}$, at baseline. Three major amino acid patterns were characterized: 1) higher loads of lysine, methionine, valine, aspartic acids, tyrosine, threonine, isoleucine, leucine, alanine, histidine, and serine; 2) higher loads of glycine, cysteine, arginine and tryptophan; 3) higher loads of proline and glutamic acid. Dietary total protein intake $(\mathrm{HR}=1.13,95 \% \mathrm{CI}=0.92-1.38$, and $\mathrm{HR}=1.00,95 \% \mathrm{CI}=$ $0.81-1.23$, in the second and third tertile, respectively) was not related to the development of prediabetes. Dietary amino acid pattern with higher load of proline and glutamic acid was related to increased risk of pre-diabetes in the fully adjusted model $(\mathrm{HR}=1.24,95 \% \mathrm{CI}=1.02-1.52 ; P$ for trend 
$=0.05)$. Conclusion: These novel data provide evidence to suggest that amino acid composition of diet may modify the risk of pre-diabetes.

\subsection{Assessment of the Refluxogenic Potential of Carbonated Beverages}

\section{B. S. X. Lim and I. A. Brownlee}

Human Nutrition Research Centre, Newcastle University (Singapore), 0501 172A Ang Mo Kio Ave 8, Singapore 567739, Singapore

Carbonated beverages have previously been inconsistently linked to gastro-oesophageal reflux occurrence. Reflux of gastric contents is linked to damage to the oesophagus and other unprotected surfaces in the airways leading to a wide range of health issues (e.g., dental erosion, oesophagitis, asthma). This study aimed to investigate the refluxogenic potential of carbonated beverage through estimation of the concentration of pepsin in saliva concentration and assessment of reflux-like symptoms post-consumption. Following ethical approval (granted by Newcastle University Faculty of Science, Agriculture \& Engineering ethics committee), ten healthy subjects were given $330 \mathrm{~mL}$ of beverage (carbonated cola, degassed cola or water) during each visit. Saliva samples were collected and symptom questionnaires were completed prior to ingestion, and at time points $0,5,15$ and 30 min after ingestion. All saliva samples were centrifuged to remove particulate matter and analysed for pepsin content using an indirect ELISA. Pepsin was detected in all samples. No significant difference was found between the salivary pepsin concentrations between the three beverages at every time point $(p>0.05)$. There were significantly higher scores of feeling of fullness, heartburn, urge to belch and frequency of belches after ingestion of carbonated cola compared to degassed cola and water at all time points except for five minutes $(p<0.001)$. The ingestion of carbonated cola does not appear to result in more frequent or larger bouts gastro-oesophageal reflux. However, ingestion of carbonated beverage was observed to evoke higher levels of reflux-related symptoms such as feelings of fullness, heartburn and belching.

\subsection{Effects of Olive Polyphenol on Prevention of Deterioration of Bone Quality}

W. Yamada ${ }^{1}$, K. Kono ${ }^{2}$, M. Ota ${ }^{1}$ and T. Yano ${ }^{1}$

1 Graduate School of Food and Nutrition Science, Toyo University, Itakura, Japan

2 Research institute for Life Innovation, Toyo University, Japan

Bone strength is determined by bone mass and quality. Bone quality is thought to encompass the structural and material properties of bone. Collagen crosslinking in bone plays important roles in keeping bone strength. Oxidative stress influences the formation of a stable bone matrix directly through the inhibition of the collagen cross-linking enzyme lysyl oxidase (LOX). Although bone density can be increased by hydroxytyrosol, a member of the olive polyphenol, the effect of hydroxytyrosol on the LOX expression in osteoblastic cells has not been understood. Thus, this study was undertaken to determine whether hydroxytyrosol could increase the LOX expression, so we tried to clarify the mechanism related to this prosess. Human osteosarcoma cells (MG-63) were seeded in culture dishes at a density of 10,000 cells $/ 3 \mathrm{~mL}$ and cultured overnight. After overnight culture, the medium was changed, and the cells were treated with hydroxytyrosol at the indicated concentrations for $48 \mathrm{~h}$. After that, we analyzed the expression of LOX mRNA by RT-real time PCR. When treated with $10 \mathrm{mM}$ hydroxytyrosol, for $48 \mathrm{~h}$, the LOX mRNA level was increased with a statistical significance $(p<0.05)$. Further investigations demonstrated that hydroxytyrosol decreased via Janus kinase 2 (JAK2) the expression of Fli1 (Friend leukemia virus integration 1) and Dnmt1, Dnmt3A, Dnmt3B $(p<0.01$ or $p<0.05)$. In conclusion, we demonstrate that hydroxytyrosol is effective to prevent the deterioration of collagen cross-linking formation.

3.109. Moderate High-Fat Diet Rich in UFA Improves Lipid Profile and Insulin Sensitivity in Normal Rats: A Critical Role of Angptls

Gang Luo, Lin Xiao, Ning Wang, Miao Hong, Xuefeng Yang and Liping Hao * 
Department of Nutrition and Food Hygiene, School of Public Health, Tongji Medical College, Huazhong University of Science and Technology, Wuhan 430030, China

* Corresponding Author

Low-carbohydrate, high-fat diets have been proven to be effective in weight loss and controlling serum lipid level, but beneficial effects of moderate high-fat diet rich in unsaturated fatty acid (UFA) among normal individuals and the potential mechanisms are seldom reported. Angptl8 is a novel liver-enriched hepatokine recognized as a potentially therapeutic target for dyslipidemia. This study explored the potential mechanisms by which moderate high-fat diet rich in UFA improved lipid profile and insulin sensitivity in normal rats, focusing on the interactions of Angptl8, lipid metabolism and insulin signaling pathway. SD rats were fed with a control Liber-Decarli liquid diet ( $35 \%$ of energy from UFA, $47 \%$ carbohydrate, $18 \%$ protein) or a moderate high-fat liquid diet ( $50 \%$ of energy from UFA, $35 \%$ carbohydrate, $18 \%$ protein) for 23 weeks. When compared with the control group, dramatically decreased serum triglyceride levels $(42 \%, p<0.01)$, less abdominal fat, perirenal fat and epididymal fat deposits were observed in moderate high-fat diet-fed rats. Moreover, liver phosphorylations of acetyl-CoA carboxylase, hormone-sensitive lipase, PI3K and Akt2 were obviously enhanced $(94 \%, 76 \%, 73 \%, 30 \% ; p<0.01)$ in the moderate high-fat diet-fed group, accompanied with up-regulated SIRT1 expression $(64 \%, p<0.01)$ but significantly reduced mRNA levels of Angptl8 and Lipoprotein lipase (62\%, 79\%; $p<0.01$ ). In long-term high-fat diet rich in UFAtreated normal rats, Angptl8 plays a critical role in improving lipid profile and insulin sensitivity, which may offer new evidence for the health benefits of low-carbohydrate, moderate high-fat diet rich in UFA in normal individuals.

\title{
3.110. Free Radical Scavenging Activity and Total Phenolic Compounds of Aquilaria Malaccensis, Ardisia Crenata and Dicranopteris Linearis
}

\author{
A. M. A. Alzubaidi ${ }^{1}$, R. N. Adnan ${ }^{1,2}$ and M. H. Salman ${ }^{3}$ \\ 1 Collage of Medicine, University of Baghdad, Baghdad, Iraq \\ 2 Faculty of Sciences, University of Baghdad, Baghdad, Iraq \\ 3 Collage of Arts, University of Baghdad, Baghdad, Iraq
}

Much attention was given to the usage of natural antioxidants to prevent oxidative damage caused by free radicals in human's body. Aquilaria malaccensis (A. malaccensis), Ardisia crenata (A. crenata) and Dicranopteris linearis (D. linearis) are three popular medicinal plants used as folk medicines. This study was undertaken to evaluate the free radical scavenging activity of these plants and their total phenolic compounds (TPC). The ABTS and DPPH assays were used to determine the free radical scavenging activity. The TPC of the extracts was determined by the Folin-Ciocalteu method. All tested extracts immediately reacted and quenched ABTS and DPPH free radicals at all concentrations $(10-320 \mathrm{~g} / \mathrm{mL}$ ) with dose and time dependence. Total antioxidant capacity (TAC) against ABTS free radical of $A$. malaccensis extract $(32.09 \pm 0.6 \%)$ was significantly more effective than A. crenata extract $(15.9 \pm 1.6 \%)$ and $D$. linearis extract $(9.9 \pm 1.9 \%)$, and comparable to ascorbic acid (33.8 $\pm 0.8 \%)$. Meanwhile, DPPH assay revealed a higher TAC of $A$. malaccensis extract $(17.9 \pm 4.1 \%)$ than ascorbic acid, A. crenata extract and D. linearis extract $(8.8 \pm 1.8 \%, 6.1 \pm 0.5 \%$ and $4.1 \pm 1 \%$, respectively). In this study, the TPC of $A$. malaccensis extract was significantly higher than $A$. crenata and D. linearis extracts which may explain its higher TAC in ABTS and DPPH assays. The results of this study reveal potent antioxidant and free radical scavenging activity of $A$. malaccensis extract which may serve as a new natural antioxidant and as a possible food supplement or in pharmaceutical applications.

\subsection{The Effects of Fructose on Uric Acid Metabolism in Rats with High Purine Diet}

\section{F. Zhong, F. Bie and A. Ma}

Affiliation not provided 
Athough fructose intake could induce hyperuricemia, the mechanisms remain unclear. We proposed the hyperuricemic model which was induced by a diet of fructose combined with yeast to investigate the effects of fructose on uric acid metabolism. Wistar male rats $(n=50)$ were randomly divided into five groups, including control group, yeast group, fructose group, yeast-oteracil potassium group and yeast-fructose group. The serum uric acid (SUA) levels at 8 weeks in the fructose group, yeast-fructose group and yeast-oteracil potassium group were significantly higher than that in control group $(p<0.05)$, but there was no difference in the urinary uric acid (UUA) levels between the control group and other groups $(p>0.05)$. The activity of serum xanthine oxidase (XOD) in the yeast-fructose group and yeast-oteracil potassium group was significantly increased $(p<0.05)$. The protein expressions of organic anion transporters 1 (OAT1) in the yeast-fructose group, fructose group and yeast-oteracil potassium group were lower $(p<0.05)$, and the protein expressions of renalspecific transporter (RST) in the yeast-fructose group were higher $(p<0.05)$ than those in the control group. The protein expressions of glucose transporter 9 (GLUT9) in the four intervention groups were higher than that in the control group, but no significant differences were observed $(p>0.05)$. These results demonstrated that fructose combined with yeast could induce hyperuricemia, which might be related to the up-regulation of renal RST expression, and the increase in the reabsorption of uric acid in the kidneys.

\subsection{The Effect of Nutritional Transition on the Differentiation of Bone Marrow Stem Cells and the} Therapeutic Effect of GLP-1

\section{W. Xia, Q. Zhang, L. Chen and J. Zhang}

Department of endocrinology, Union Hospital, Tongji Medical College, Huazhong University of Science and Technology, Wuhan 430022, China

Background: Catch up growth is associated with glucose and lipid metabolism disorders, that can lead to osteoporosis and related fractures. Glucagon-like peptide-1 receptor agonists, such as liraglutide, exert an anabolic effect on bone in insulin-resistant mice, but the mechanism for the action remains unclear. We established an animal model of catch-up growth to explore the actions of GLP1 on bone mass and structure.

Methods: 30 male C57BL/6 mice were randomly divided into the normal diet (ND, $n=10)$, the catch-up growth model $(\mathrm{RH}, \mathrm{n}=10)$ and the catch-up growth model +GLP-1 ( $\mathrm{RH}+\mathrm{GLP}-1, \mathrm{n}=10)$ groups. Liraglutide was subcutaneously administered ( $200 \mathrm{ug} / \mathrm{kg}$, twice a day, for four weeks). After killing, the tibia and fibula were removed for bone histomorphometry; bilateral femoral bone marrow mesenchymal stem cells were collected to evaluate their differentiation ability. Results: GLP-1 administration decreased the weight of visceral fat tissue, body weight, the ratio between them, and blood lipids of the RH group. GLP-1 decreased triglyceride levels, and fatty acids in the liver, visceral fat tissue, subcutaneous fat tissue and skeletal muscle tissue. Compared to the ND group, GLP-1 secretion decreased in the RH group, but noticeably increased after GLP-1 administration. The areas under curves of the intraperitoneal glucose tolerance test and insulin tolerance test and HOMA-IR in the RH + GLP-1 group significantly declined. GLP-1 reversed deteriorated tibial and fibular bone structure in RH mice and induced osteoblastic differentiation. Conclusion: The decreased ability of osteoblastic/osteogenesis differentiation of MSCs in RH mice led to impaired bone formation and GLP-1 intervention reversed the damage and enhanced insulin sensitivity.

\subsection{The Effects of Hydration Statuses on Cognitive Performances among College Students: A Randomized Controlled Trial (RCT)}

Jianfen Zhang 1,2, Na Zhang 1,2, Songming Du ${ }^{3}$, Hairong He 1,2, Yifan Xu ${ }^{1,2}$, Hao Cai 1,2, Xiaohui Guo ${ }^{1,2}$ and Guansheng Ma 1,2,*

1 Department of Nutrition and Food Hygiene, School of Public Health, Peking University Health Science Centre; 38 Xue Yuan Road, Haidian District, Beijing 100191, China

2 Laboratory of Toxicological Research and Risk Assessment for Food Safety, Peking University Health Science Centre; 38 Xue Yuan Road, Haidian District, Beijing 100191, China 3 Chinese Nutrition Society; 6 
Guang An Men Nei Street, Xicheng District, Beijing 100053, China

* Corresponding Author

Background: Dehydration may affect cognitive performance as water accounts for $75 \%$ of brain mass.

Methods: A randomized controlled trial is designed and will be implemented among 64 college students aged 18-23 years from Baoding, China. The study consists of three periods. Subjects will be asked to fast overnight for $12 \mathrm{~h}$. Then, they will have a restricted supply of water for $24 \mathrm{~h}$. The first morning urine and blood sample will be collected and measured. MRI sequences including fMRI, ASL and 3D BRAVO will be taken to observe the changes in the brain. Cognitive performance and mood will be tested by questionnaires. Then, subjects will have water supplementation. The first morning urine, blood samples, MRI scans, cognitive performance and mood will be measured. Subjects will be randomly assigned into four groups: water supplementation group 1, 2, 3 with 500, 1000 and $1500 \mathrm{~mL}$, respectively (WS group 1, 2, 3) and no water supplementation group (NW group). Subjects in the WS groups will drink water within $15 \mathrm{~min}$, while those in the NW group will not drink any fluid. Ninety minutes later, urine, blood samples, MRI scans, cognitive performance and mood will be measured. A mixed model of repeated measures ANOVA will be used to study the effects of hydration status on cognitive performance. Conclusion: This is the first study to investigate the effects of dehydration and rehydration on cognitive performances and the changes of brain using MRI among young adults in China.

Keywords: water restriction; dehydration; rehydration; cognitive performances; magnetic resonance imaging

\subsection{Effects of Meal Intervention Combined with Walking on Exercise Ability and Physique of Middle-} Aged and Elderly People

Qihe Chen, Ruixue Mao, Meihong Xu, Rui Fan and Yong Li *

Department of Nutrition and Food Hygiene, School of Public Health, Peking University, Beijing, China

* Corresponding Author

We aimed to investigate the effects of vigorous walking and diet intervention on the exercise capacity and physical fitness of middle-aged and elderly people. According to the different physical conditions of middle-aged and elderly people, the data were divided into $93 \mathrm{~m} / \mathrm{min}, 100 \mathrm{~m} / \mathrm{min}, 107$ $\mathrm{m} / \mathrm{min}, 110 \mathrm{~m} / \mathrm{min}$, and $114 \mathrm{~m} / \mathrm{min}$ by comparing their own physical condition before and after the test. Blood pressure, heart rate, body weight, waist circumference, hip circumference, systolic blood pressure, diastolic blood pressure, BMI and other data were collected in the intervention group with the maximum exercise load of 50\%. Compared with the baseline, the 6-min walking distance, cardiac recovery rate, systolic blood pressure, diastolic blood pressure, body weight, hip circumference and BMI of the subjects were significantly improved $(p<0.05)$. There were different degrees of improvement. Vigorous walking and dietary intervention have a significant effect on the exercise capacity and physical fitness of the elderly, and it is suggested that they be promoted.

\subsection{The Association between Serum EPA/AA Ratio and Autonomic Nervous Function during a Simple Standing-Up Test}

Mika Yamaneki, Hiroto Tanaka, Hideyuki Sasaki and Mikio Arita

Department of Internal Medicine, Wakayama Medical University, Kihoku Hospital, Wakayama, Japan

Introduction: It has been revealed that the ratio of eicosapentaenoic acid (EPA) to arachidonic acid (AA) is important for the prevention of cardiovascular disease. On the other hand, the improvement of the autonomic nervous system is associated with the cause of hypertension. In this study, we investigated whether the serum EPA/AA ratio was associated with autonomic function during a simple standing-up test in healthy individuals. Methods: We enrolled 343 healthy subjects (mean age $61.8 \pm 9.1$ years). The serum level of PUFA, including EPA, docosahexaenoic acid (DHA), 
and AA, was measured. Healthy subjects were divided into subgroups according to age. The subjects performed a simple standing-up test: they sat in a chair for $2 \mathrm{~min}$, stood up and remained standing for $2 \mathrm{~min}$, then sat down and remained seated for $1 \mathrm{~min}$. During the test, brachial BP and pulse rate (PR) were measured every minute with an automated cyclometric BP device (Circlemates; Crosswell, Kanagawa, Japan). The serum EPA/AA ratio and CVRR, CCVHF, and CCVLF, respectively showed a significant negative relation. Similarly, serum DHA and CVRR, CCVHF, and CCVLF, respectively showed a significant negative relation. However, because autonomic nervous function factor and age showed a significant negative relation, an influence by age was suggested. Thus, at age 70 years, the serum EPA/AA ratio and $\triangle \mathrm{LF} / \mathrm{HF}$ and the mean pulse rate showed a significant negative relation. Conclusion: The association between the serum EPA/AA ratio and autonomic nervous function was shown. Especially, at age 70 years, the increase of serum EPA/AA contributed to the suppression of sympathetic variation.

\title{
3.116. The Development of a Database to Measure Nitrate, Nitrite, and Nitrosamines for Use with a FFQ: A Systematic Review
}

\author{
N. F. McMahon ${ }^{1}$, T. G. Pavey ${ }^{2}$, B. Desbrow ${ }^{3}$ and M. D. Leveritt ${ }^{1}$ \\ 1 School of Human Movement and Nutrition Sciences, University of Queensland, St. Lucia, QLD, Australia \\ 2 School of Exercise \& Nutrition Sciences, Queensland University of Technology, Kelvin Grove, QLD, \\ Australia \\ 3 School of Allied Health Sciences, Griffith University, Gold Coast, QLD, Australia
}

Background/Aims: The use of dietary nitrate $\left(\mathrm{NO}_{3}^{-}\right)$supplements has become more popular amongst athletes attempting to enhance sport performance. Although the most common type of nitrate supplementation is beetroot juice, recent research suggests that ingestion of nitrate-rich vegetables is equally as effective. The substantial variability in nitrate concentrations in food highlights the need for a validated dietary assessment tool to accurately determine $\mathrm{NO}_{3}{ }^{-}$and nitrite $\left(\mathrm{NO}_{2}{ }^{-}\right)$intake. The purpose of this systematic review was to create a comprehensive database to measure the concentrations of $\mathrm{NO}_{3}^{-}, \mathrm{NO}_{2}{ }^{-}$, and nitrosamines in foods and beverages. Methods: Studies were included if extractable data was available for foods or beverages. Where multiple reference sources were available, items were grouped into categories by type, and coded based on publication information so the user can filter values based on their own specific requirements. Results: The database contains a total of $3483 \mathrm{NO}_{3}^{-}, 2135 \mathrm{NO}_{2}^{-}$, and 954 nitrosamines individual food and beverage estimates from 59 different countries $(n=274$ studies). There are 2682 vegetable samples, 714 protein foods, 535 processed meats, 437 dairy products, and 402 baby products. Conclusions: This database enables the quantification of dietary intakes of $\mathrm{NO}_{3}^{-}, \mathrm{NO}_{2}^{-}$, and nitrosamines from food and beverages for use in both public health and clinical settings. Understanding dietary information may help lead to the development of recommendations for dietary $\mathrm{NO}_{3}$ - supplementation, specifically in relation to (1) optimal dosing strategies; (2) which population is most likely to benefit; and (3) under which conditions dietary nitrates are likely to be most effective for enhancing sports performance.

\subsection{An Observational Examination of the Prevalence, Attitudes, and Beliefs of Dietary Nitrate Supplement Usage among Active Adults}

\section{N. F. McMahon ${ }^{1}$, T. G. Pavey ${ }^{2}$, B. Desbrow ${ }^{3}$ and M. D. Leveritt ${ }^{1}$}

1 School of Human Movement and Nutrition Sciences, University of Queensland, St. Lucia, QLD, Australia

2 School of Exercise \& Nutrition Sciences, Queensland University of Technology, Kelvin Grove, QLD, Australia

3 School of Allied Health Sciences, Griffith University, Gold Coast, QLD, Australia

Background/Aims: Consumption of dietary nitrate supplements has gained popularity amongst athletes in an attempt to enhance performance. However, it is still unclear if the use of dietary nitrate $(\mathrm{DN})$ as a form of supplementation is widespread and how the knowledge from research studies is being translated. The objective of this study was to assess the prevalence, frequency of use, 
knowledge, and the attitudes and beliefs of DN supplements among active adults. Methods: Individuals ( $\geq 16 \mathrm{y}$ ) completed a 42-item online questionnaire designed to assess the attitudes and beliefs around DN consumption. Results: 1404 active adults (66.4\% female) participated in the survey. In total, $11.9 \%$ of survey respondents reported to have consumed DN ('users') in the past, most commonly in the form of beetroot juice $(31.3 \%)$. Users had a greater understanding of usage recommendations compared to non-users $(p<0.01)$. Despite this, more than two-thirds $(69.4 \%)$ of users could not correctly identify the correct timing of intake relative to performance time to best improve performance, and the majority of users $(82.3 \%)$ were unsure of the contraindications to oral consumption of DN supplements. Conclusions: The results outline the gap in knowledge that exists between current consensus recommendations and its translation to practice. Since DN supplements are well-documented to improve sports performance, they are severely under-utilised. Educational messages that target DN consumption should be targeted towards nutritionists, coaches, and exercise physiologists to bridge the gap between knowledge-to-practice.

\subsection{Assessment of Energy Balance and Nutrition Attitudes of Collegiate Athletes before, during, and after Training Days}

Anna Monica B. Dumalaog, Mary Nicole I. Grecia and Patricia Bernadette A. Palencia

Department of Food Science and Nutrition, University of the Philippines Diliman, Philippines

Student-athletes from weight-class sports are exposed to high pressure conditions due to heavy academic load and frequent weigh-ins. Collegiate Varsity Members drastically change food intake before weigh-ins to meet specific weight classes. Such conditions lead to the development of eating disorders and binge diets, and as a result, excess and severe depletion of nutrient stores may pose adverse health effects later on, alongside decreased athletic performance. Thus, proper nutrition management is needed. Study participants from the University of the Philippines Judo Team $(n=20)$ completed an adapted Nutrition Knowledge, Attitudes, and Practices Questionnaires, 3-day nonconsecutive 24-h estimated food records, and 24-h physical activity diaries before, during, and after the training days. Through the one tailed $t$-test $(p=0.05)$, a significant difference was recorded between the mean caloric intake of participants from training to post training days $(t=-1.967)$. Meanwhile, the caloric expenditure of participants were significantly higher during training days, compared to no training $(t=5.03)$ and post training $(t=6.42)$ days. This difference in caloric intake and expenditure may lead to athletes developing lifestyle diseases such as obesity if they decide not to pursue Judo in later life. Pearson correlation coefficient test shows no correlation between nutrition knowledge scores and energy balance $(R=0.00)$. Weak positive correlation was found between the score for sports and nutrition attitudes and energy balance. $(R=0.33)$. Present data suggest a more positive energy balance, or less negative energy balance can be weakly associated with increased preference towards the power and performance model of sports participation.

\subsection{Gestational Weight Gain Is Associated with Delayed Onset of Lactogenesis in the TMCHC Study: An Observational Cohort Study in Wuhan, China}

Li Huang 1, Xi Chen 1, Yu Zhang 1, Chunrong Zhong 1, Weiye Wang 1, Qian Li 1, Xiating Li 1, Heng Yin ${ }^{2}$, Xuefeng Yang ${ }^{1}$, Liping Hao ${ }^{1}$, Mei Xiao ${ }^{2}$ and Nianhong Yang 1 ,*

1 Department of Nutrition and Food Hygiene, Hubei Key Laboratory of Food Nutrition and Safety, Ministry of Education (MOE) Key Lab of Environment and Health, School of Public Health, Tongji Medical College, Huazhong University of Science and Technology, China

2 Department of Obstetrics and Gynaecology, Hubei Maternal and Child Health Hospital, China

* Corresponding Author

To assess the relationship between gestational weight gain (GWG) throughout pregnancy and risk of delayed onset of lactogenesis (OL), we used data from Tongji Maternal and Child Health Cohort (TMCHC), a prospective cohort of pregnant women at 8-16 weeks of gestations in Wuhan, China from 2013-2016. GWG was assessed by the last available weight measurement during pregnancy and the self-reported pre-pregnancy weight at enrollment. Information about age, 
ethnicity, education, income, smoking, alcohol consumption and parity was obtained from a structured questionnaire, and height was measured at enrolment when they had their first antenatal visit. Delayed OL was assessed by maternal report of breast fullness within days 4-7 postpartum. Women were determined to be delayed OL if they reported milk ejection $>72 \mathrm{~h}$ postpartum. Chisquare tests or one-way analysis of variance (ANOVA) were used to compare demographic characteristics of pregnant women by GWG categories. Logistical regression analysis was used to assess the association between GWG and delayed OL and Odd rations (ORs) were estimated. The prevalence of delayed OL was $18.7 \%$. Compared to the gaining weight $<13.0 \mathrm{~kg}$ group, the ORs of delayed OL for GWG of 13.0 15.7, 15.8 18.7 and $\geq 18.8$, were 1.18 (95\% CI: 0.89, 1.51), 1.41 (95\% CI: $1.07,1.84)$, and 1.34 (95\% CI: 1.02, 1.78) after adjustment for the demographic and clinical information. In conclusion, women with excessive GWG throughout pregnancy are more likely to suffer from delayed OL and the management of GWG should be emphsized.

\subsection{Effect of Dehydration and Rehydration on Short-Term Cognitive Performances among Adult Male College Students in Hebei, China}

\section{N. Zhang, S. Du, J. Zhang and G. Ma}

Objective: To investigate the effects of dehydration and rehydration on cognitive performances. Methods: a total of 12 subjects participated in the trial. On the day before the trial, subjects were fasted from 20:00 for $12 \mathrm{~h}$. On the first day, morning urine samples were collected to determine osmolality, concentrations of potassium, sodium, and chloride. Height, weight and blood pressure were measured. A POMS questionnaire and cognitive performance test were completed. Elbow venous blood was collected to determine blood osmolality, concentrations of potassium, sodium, and chloride, and blood sugar. Then, subjects were not allowed to drink water from 20:00 for $36 \mathrm{~h}$. On the second day, subjects were given specified solid food and all food was weighed to assess fluid intake from food, and $24 \mathrm{~h}$ urine was collected to evaluate urine output and urine osmolality. On the third day, all measurements were conducted again at 8:00. Then, subjects were asked to drink $1500 \mathrm{~mL}$ pure water from 8:30 to 8:45. At 9:30, all measurements were conducted again.

Results: Compared with baseline, the hydration status of subjects was different with statistical significance $\left(\chi^{2}=34.579, p<0.001\right)$, and subjects had less energy, showed esteem-related effects, lower total digit span score and higher error rate in the dose work test after having not drank water for 36 h. After water supplementation, the hydration status of subjects was different with statistical significance $\left(\chi^{2}=20.308, p<0.001\right)$, and subjects were less fatigued, had lower TMD, higher digit span forward, backward and total score in the digit span test, a more correct number in the digit symbol substitution test, higher reading speed and mental work ability index in the dose work test, and quicker reaction time in the color-word stroop test.

Conclusion: Dehydration, without water-drinking for $36 \mathrm{~h}$, has negative effects on energy, esteem-related effects, short-term memory ability and attention span. Water supplementation has beneficial effects on hydration status, fatigue levels, TMD, short-term memory, digit symbol substitution test, attention span, and reaction time.

\subsection{The Effects of Aerobic Exercise on the Management of Type-2 Diabetics: A Randomized Control Trial}

Rui Fan, Meihong Xu and Yong Li

Affiliation not provided

Glycemic control and weight reduction are primary goals for the management of type 2 diabetes mellitus (T2DM). Effective management cannot be achieved by an appropriate diet alone. Our study aimed to evaluate the effects of aerobic exercise and develop a reasonable management plan for T2DM patients. A randomized control trial was carried out among elder T2DM patients. One hundred and six were selected from 134 patients between 50 and 70 years, and participated in a single-blinded 30-day centralized management. Participants in the program were randomly assigned into one of the two groups: usual care group (control group, only systematic diet plans), aerobic 
exercise group (systematic diet plans combined with aerobic exercise). Anthropometric, blood glycemic and blood pressure were measured and questionnaires were analyzed. For the 30-day intervention, the significant decrease in weight, BMI, FPG, 2h-PG and systolic pressure were shown among the two groups. Compared to the control group, the aerobic exercise group had a bigger reduction in FPG (mean difference (MD): -0.9 mmol/L; 95\% CI: $-3.7,1.9$ ), 2h-PG (MD: $-2.33,95 \%$ CI: $-5.54,2.43$ ), systolic Pressure (MD): $-0.51 \mathrm{mmHg}$; 95\% CI: $-1.36,0.34$ ), and diastolic pressure (MD): $-3.96 \mathrm{mmHg}$; $95 \% \mathrm{CI}:-7.07,-0.87)$. In addition, with the rise of intervention time, a sustained decline in FPG and 2h-PG was observed in the aerobic exercise group and its DSQL (Diabetes specific quality of life scale) scores were relatively lower. In conclusion, aerobic exercise had significant effects on controling blood glucose. Our study provided some supportive evidence recommending the comprehensive management of diabetics.

\subsection{Investigation on the Prevalence and Influences of Thyroid Nodules in Adults in Tianjin}

L. Fan ${ }^{1}$, L. Tan ${ }^{1}$, Y. Chen ${ }^{1}$, C. Du ${ }^{1}$, W. Wang ${ }^{1}$, M. Gao ${ }^{1}$, Y. Zhang ${ }^{1}$, W. Chen ${ }^{1}$, J. Shen ${ }^{2}$ and W. Zhang ${ }^{1, *}$

1 The Department of Nutrition and Food Hygiene, Tianjin Medical University, Tianjin, China

2 Department of Sanitary Chemistry, School of Public Health, Tianjin Medical University, Tianjin, China

* Corresponding Author

In recent years, the prevalence of thyroid nodules (TNs) has shown a rising trend. This study aimed to understand the prevalence of TNs in Tianjin and possible factors impacting on TNs. In this study, simple random sampling was used to investigate residents aged 18 years or older from Tianjin. All of the subjects accepted thyroid ultrasonography, physical examination and questionnaires. Spot urine samples were collected. Fasting venous blood samples were collected to determine thyroid hormone (TSH), thyroid autoantibody TPOAb and TGAb. Subjects were divided into case and control groups according to whether they suffered from TNs, then potential influencing factors of thyroid nodules including sex, age, iodine status, thyroid volume, TSHTPOAbTGAb and some living habits were analysed. In total, 2647 adults were surveyed. The total TNs prevalence was $26.7 \%$, prevalence of women was higher than that of men $(p<0.05)$. TN prevalence increased with age $(p<0.001)$ and was lower in the population with a higher urinary iodine concentration $(p<0.05)$. The positive rate of TPOAb and goiter rate in the case group were higher than that in the control group $(p<0.05)$. The thyroid volume in the case group was larger than that in the control group $(p<0.001)$. Other factors that may influence TNs included high blood pressure, iodized salt, menopause, seafood intake, and education levels. Multivariate logistic regression analysis showed that iodine deficiency, female, aging, smoker were risk factors for TNs. In conclusion, adults in Tianjin showed inadequate iodine status. TNs prevalence in Tianjin was high. Factors that may be associated with TNs included gender, age, iodine status and smoking.

\subsection{An Exploration of Comprehensive the Intervention Model Including Exercise, Diet and Health} Education in the Management of Type 2 Diabetes Mellitus

Ruixue Mao, Rui Fan and Yong $\mathrm{Li}^{*}$

Department of Nutrition and Food Hygiene, School of Public Health, Peking University, Beijing, China * Corresponding Author

Objective: To explore the effects of comprehensive interventions on type 2 diabetes mellitus (T2DM) patients, which leads to the exploration of proper comprehensive approaches to T2DM management.

Methods: A single-blinded prospective study was carried out in 28 adults of 50-75 years old diagnosed as T2DM. Participants received $30 \mathrm{~min}$ of fast walking intervention at $50 \%$ of their maximum heart rate, structured dietary treatment with added naked oat, and systematic intensive health education for 30 days. During 30 days of centralized intervention, physical examinations including weight, body composition, waist circumference, hip circumference, and blood pressure 
were carried out at baseline as well as at the end of intervention. Meanwhile fasting blood glucose (measured before exercise), heart rate recovery, $2 \mathrm{~h}$-postprandial blood glucose (measured after exercise), and bedtime blood glucose were determined every 5 days from baseline.

Results: Through multiple interventions with individualized aerobic exercise, the mean weight, hip circumstance, percentage of body fat, visceral fat rate and BMI of participants decreased significantly $(p<0.05)$. Meanwhile, $57.6 \%$ of participants showed improvements in heart rate recovery, indicating potential heart lung function improvements. Moreover, levels of fasting blood glucose, $2 \mathrm{~h}-$ postprandial blood glucose, and bedtime blood glucose decreased significantly $(p<0.05)$ after interventions with 2-h postprandial blood glucose levels below $10 \mathrm{mmol} / \mathrm{L}$.

Conclusion: The combination of physical exercise, structured diet with naked oat and health education showed positive effects on glycemic control and athletic ability with potential improvements in heart lung function for patients with type 2 diabetes. In this study, we established a proper model for T2DM comprehensive management, which was the basis of diabetes mellitus disease management.

Keywords: T2DM; physical exercise; structured diet; health education

\subsection{Meta-Analysis Update}

\section{N. F. McMahon ${ }^{1}$, T. G. Pavey ${ }^{2}$, B. Desbrow ${ }^{3}$ and M. D. Leveritt ${ }^{1}$}

1 School of Human Movement and Nutrition Sciences, University of Queensland, St. Lucia, QLD, Australia

2 School of Exercise \& Nutrition Sciences, Queensland University of Technology, Kelvin Grove, QLD, Australia

3 School of Allied Health Sciences, Griffith University, Gold Coast, QLD, Australia

Background/Aims: The use of dietary nitrates has become more popular amongst athletes attempting to enhance performance. The objective of this systematic review and meta-analysis was to evaluate the effect of dietary nitrate $\left(\mathrm{NO}_{3}{ }^{-}\right)$supplementation on endurance exercise performance.

Methods: Relevant databases, Cochrane Library, Embase, PubMed, Ovid, Scopus and Web of Science were searched from inception to September 2017. Studies were included if a placebo versus dietary nitrate-only supplementation protocol was able to be compared, and if quantifiable measures of exercise performance were $\geq 30 \mathrm{~s}$ (for a single bout of exercise or the combined total for multiple bouts).

Results: Data from the 109 trials was extracted for inclusion in the meta-analysis. A randomeffects meta-analysis was conducted for time trial (TT) ( $n=48)$, and time to exhaustion (TTE) ( $n=23)$. Pooled analysis identified a trivial, but NS effect in favour of dietary $\mathrm{NO}_{3}{ }^{-}$supplementation for TT (effect size $(E S)=-0.11,95 \% \mathrm{Cl}=-0.23-0.02, p>0.05$ ). TTE trials had a small to moderate statistically significant effect in favour of dietary $\mathrm{NO}_{3}{ }^{-}$supplementation $(\mathrm{ES}=0.32,95 \% \mathrm{Cl}=0.15-0.49, p<0.01$ ). No significant heterogeneity was detected in the meta-analysis.

Conclusions: Dietary $\mathrm{NO}_{3}{ }^{-}$supplementation is likely to elicit a positive outcome when testing endurance exercise capacity, but likely to be less effective for time-trial performance. Future research should assess optimal dosing strategies, which population is most likely to benefit, and under which conditions dietary nitrates are likely to be most effective for performance.

Funding source(s): N/A.

\subsection{The Association of MTHFR C677T Gene Polymorphism and Incidence of Breast Cancer: A Systematic} Review and Meta-Analysis

A. Sato ${ }^{1,2}$, M. Ota ${ }^{2}$, Y. Sekine ${ }^{1}$ and T. Yano ${ }^{2}$

1 Graduate School of Medical and Pharmaceutical Sciences, Chiba University, Japan

2 Research Institute of Life Innovation, Toyo University, Tokyo 112-8606, Japan

Methylenetetrahydrofolate reductase (MTHFR) is an essential enzyme involved in folate metabolism. MTHFR gene polymorphism (C677T), comprised of wild (CC), hetero (CT), and homo 
(TT) genotypes, has been implicated in several types of cancer. However, the association of MTHFR C677T polymorphism and carcinogenesis is not clear. This study was undertaken to meta-analytically determine which organ is susceptible to carcinogenesis caused by the genetic polymorphism and to clarify the association of the polymorphism and the incidence of cancer in the determined organ. Case-control and/or cohort studies were identified through the PubMed database concerning the association between MTHFR C677T gene polymorphism and cancer incidence to identify potentially eligible articles. Odds ratios (ORs) and 95\% confidence intervals (CIs) were utilized to estimate the strength of the associations. Besides, a heterogeneity test and publication bias evaluation were conducted in current meta-analysis as well. Ultimately, 198 reports were assessed for eligibility; among them, we focused on breast cancer. Forty-seven reports were found on breast cancer, and 20 reports which met the selection criteria were included in this meta-analysis. When only the MTHFR C677T polymorphic CC type and TT type were compared for breast cancer incidence (TT vs. CC: OR $=1.36$ [95\% CI, 1.00-1.85], $p=0.05$ ) in postmenopausal women, a significant difference was recognized. Our finding indicates that MTHFR C677T gene polymorphism may be a susceptible factor to breast cancer mainly in postmenopausal women.

\title{
3.163. The Effects of Starvation on Genes Expression in a Rat Model
}

C. P. Hsu ${ }^{1,2}$ and T. H. Hsiao ${ }^{1}$

1 Department of Surgery, Taichung Veterans General Hospital, Taichung, Taiwan

2 School of Medicine, National Yang-Ming University, Taipei, Taiwan

To observe the changes of gene expressions after starvation, adult male rats (Sprague-Dawley) were divided into three groups (two rats in each group) according to the amount and type of food feeded: regular diet (RD) group ( $22 \mathrm{gm} /$ day, $28.5 \mathrm{Kcal} /$ day, $4.5 \%$ fat), limited diet (LD) group (15 gm/day, $20.5 \mathrm{Kcal} /$ day, $4.5 \%$ fat), and high-fat diet (HFD) group (15 gm/day, $67.5 \mathrm{Kcal} /$ day, 34.9\% fat). During the study period, body weights (BW) were recorded every week. Blood collection was conducted at the end of the 12th week when a 15\% BW difference had been reached between the RD and LD groups, then at the end of the 13th week after starvation for one week. RNAs were retrieved for Gene Chip hybridization, and the microarray data analyzed. The initial BWs of RD/LD/HFD groups were $239.5 \mathrm{gm} / 232.5 \mathrm{gm} / 246.0 \mathrm{gm}$, respectively. At the end of the 12th week, the BWs of $\mathrm{RD} / \mathrm{LD} / \mathrm{HFD}$ groups were $310.5 \mathrm{gm} / 265.5 \mathrm{gm} / 366.0 \mathrm{gm}$, respectively. While at the end of the 13th week, the BWs of RD/LD/HFD groups were $244.0 \mathrm{gm} / 198.0 \mathrm{gm} / 340.5 \mathrm{gm}$, respectively. We identified 168 up-regulated genes and 255 down-regulated genes associated with starvation in all three groups, and the top 10 up-regulated genes (Kcns2, Hipk3, Mamdc4, Dpf1, Clip2, R3hdm4, Pax2, P2rx1, Zyx, RGD1564129; $p<1.0 \times 10^{-10}<1.0 \times 10^{-18}$ ), and down-regulated genes (Mpeg1, Treml4, Emr4, Papss2, Gngt2, Cx3cr1, Clec4a3, Tcf712, Cd59, Flnb |LOC100911261; $p<1.0 \times 10^{-5}<1.0 \times 10^{-9}$ ) were identified for further functional study.

\subsection{Association of Dietary Quality with Morningness-Eveningness and Genetic Factors among Japanese College Students}

\author{
K. Yamamoto ${ }^{1}$, M. Ota ${ }^{1}$ and T. Yoshizaki ${ }^{2}$ \\ 1 Graduate school of Food and Nutritional Science, Toyo University, Gunma, Japan \\ 2 Food and Nutritional Science, Toyo University, Gunma, Japan
}

Food group intakes have been associated with morningness-eveningness, which also can be associated with genetic polymorphisms in circadian clock-related genes such as period3 (per3) and circadian locomotor output cycles kaput (clock). The aim of our study was to elucidate the association between dietary quality, morningness-eveningness, and distribution of polymorphisms in per3 and clock. Forty-four healthy college students completed a questionnaire that included demographic characteristics, dietary intakes over the previous 1 month, the Pittsburgh Sleep Quality Index, and the Morningness-Eveningness Questionnaire (MEQ). Saliva samples were obtained to assess polymorphisms in per3 VNTR and clock 3111T/C SNP. Dietary quality was based on the adherence to 
the Japanese Food Guide Spinning Top (food guide score, 0-70 points), which consists of grain, vegetable, fish and meat dishes, milk and milk products, fruits, total energy, snacks, and alcoholic beverages. A higher score reflects a higher variety of food. Participants were separated into two groups (High group, Low group) according to the median food guide score (40 points) to compare each variable. The food guide scores in the high and low group were $34.8 \pm 5.3$ and $47.2 \pm 5.6$ points, respectively. Sleep duration and the MEQ score in the high group were significantly lower and higher than those in the low group, respectively. Morningness-eveningness was significantly associated with adherence to the Japanese Food Guide Spinning Top, though polymorphisms in per3 VNTR and clock 3111T/C SNP were not. Further studies are needed to explore whether changes in morningnesseveningness have a specific effect on dietary diversity.

\subsection{Short-Term Effect of Plant-Based Nutrients on Transcriptomic Profiling: A Systematic Review of Human Clinical Trials}

\section{N. Jamshidi ${ }^{1}$, N. Mantri ${ }^{2}$ and M. M. Cohen ${ }^{1}$}

1 School of Health and Biomedical Sciences, RMIT University, Victoria, Australia

2 School of Science, RMIT University, Victoria, Australia

The association of nutrients with genes or nutrigenomics has been extensively studied to determine the role of dietary nutrients in metabolic function. The advent of transcriptomics has been instrumental in understanding the modulation of gene expression and the molecular basis for the effects of various diets and plant-based nutrients in diabetes, and metabolic syndrome. To review the evidence on the short-term effect of plant-based nutrients on gene expression profiling from human intervention studies, electronic databases were systematically searched using search terms such as "transcriptome", "RNA-seq", "plants" or "extract". Studies were included if they involved human clinical studies of single plant-based nutrients and reported short-term outcomes related to transcriptome or gene expression profiling. The impact of acute dietary supplementation with natural plant oil extracts on whole genome transcriptome was reported in four clinical studies and plant herb extracts in five studies. Gene expression varied at different times during a 24-h period with half of the included studies reporting changes in gene expression associated with immune response, inflammatory, metabolic and lipid metabolism. Emerging evidence from transcriptome profiling suggests that plant products may have protective short-term effects mediated through changes in gene expression related to inflammation, insulin-sensitivity, immune response as well as atherogenic genes implicated in diabetes and metabolic syndrome. Further studies are required to validate and further understand the health impacts of dietary plants on gene expression and function.

\subsection{Diet, Biochemical Markers and Interaction with FTO Gene Polymorphisms in Malaysian Adults}

S. R. Mitra ${ }^{1}$, P. Y. Tan ${ }^{1}$ and Farahnaz Amini ${ }^{2}$

1 School of Biosciences, Faculty of Science, University of Nottingham Malaysia Campus, Malaysia

2 School of Healthy Aging, Medical Aesthetics \& Regenerative Medicine, UCSI University, KL Campus, Malaysia

Gene variations can interact with diet and modulate phenotypic traits in individuals. This study aimed to examine the interaction between dietary parameters and FTO gene variations on biochemical markers in Malaysian adults. Mean age $( \pm S E)$ for Malays $(n=85)$, Chinese $(n=41)$ and Indians $(\mathrm{n}=50)$ were $39.1 \pm 1.2 \mathrm{y}, 42.6 \pm 1.9$ y and $45.7 \pm 1.6$ y respectively. Fasting blood samples ( $\mathrm{n}$ $=124$ ) were collected and $24 \mathrm{~h}$ dietary recall (triple-pass method) was used to record habitual dietary intake. Our results revealed that, although Indians had significantly higher consumption of fibre compared to Chinese and Malays (13.2 $\mathrm{g} \pm 1.0$ vs. $11.8 \mathrm{~g} \pm 1.2$ and $9.0 \mathrm{~g} \pm 0.6, p=0.005)$; higher percentage energy from carbohydrate $(50.2 \pm 1.2 \mathrm{vs} 44.5 \% \pm 1.2$ and $45.6 \% \pm 1.2, p=0.01)$; lower dietary cholesterol (149.1 $\mathrm{mg} \pm 20.1 \mathrm{vs.} 344.8 \mathrm{mg} \pm 44.0$ and $257.5 \mathrm{mg} \pm 25.0, p=0.001)$ and lower percentage energy from protein $(13.0 \% \pm 0.5$ vs. $17.8 \% \pm 0.8$ and $14.6 \% \pm 0.5, p=0.001)$, they had significantly higher HOMA-IR $(3.85 \pm 0.7,1.45 \pm 1.2$ and $1.78 \pm 0.2, p=0.001)$, higher total cholesterol/HDL 
cholesterol ratio $(4.07 \pm 0.2$ vs. $3.51 \pm 0.3$ and $3.58 \pm 0.1, p=0.001)$, higher hsCRP levels $(7.67 \mathrm{mg} / \mathrm{L} \pm$ $1.4,2.68 \mathrm{mg} / \mathrm{L} \pm 0.9$ and $5.04 \mathrm{mg} / \mathrm{L} \pm 0.9, p=0.004)$ and lower HDL cholesterol levels $(1.34 \mathrm{mmol} / \mathrm{L} \pm$ 0.04 vs. $1.73 \mathrm{mmol} / \mathrm{L} \pm 0.1$ and $1.61 \pm 0.05 \mathrm{mmol} / \mathrm{L}, p=0.001$ ). Indians expressed significantly higher frequency of both heterozygous and homozygous mutant gene variants in all three SNPs. Individuals carrying GG genotype of rs9930501 had significantly higher hsCRP levels when consuming low Vitamin E compared to those with high Vitamin E intake $(11.8 \mathrm{mg} / \mathrm{L} \pm 2.3 \mathrm{vs} .3 .6 \mathrm{mg} / \mathrm{L} \pm 2.2, p=0.05)$. Similar results were found in $\operatorname{rs} 9930506(p=0.05)$ and $\operatorname{rs9932754}(p=0.05)$ as well. A to $\mathrm{G}$ polymorphism in rs9930501 and rs9930506 and T to C polymorphism in rs9932754 may have contributed to obesity phenotypes in our Indian participants.

\subsection{Effect of DBP Gene Polymorphisms on the Serum Vitamin D in the Chinese Rural Population: The RuralDiab Study}

X. Liu ${ }^{1}$, Y. $\mathrm{Li}^{1}$, G. Wang ${ }^{1}$, Z. Mao ${ }^{1}$, S. Yu ${ }^{2}$, F. Yu ${ }^{2}$, W. Li ${ }^{2}$ and C. Wang ${ }^{1, *}$

1 Department of Epidemiology and Biostatistics, College of Public Health, Zhengzhou University, Zhengzhou, China

2 Department of Nutrition and Food Hygiene, College of Public Health, Zhengzhou University, Zhengzhou, China

* Corresponding Author

The purpose of this study was to explore the association of vitamin D binding protein (DBP) gene polymorphisms with the serum vitamin $\mathrm{D}[25(\mathrm{OH}) \mathrm{D})]$ level in the Chinese rural population. A total of 1544 participants aged 16-74 years from the RuralDiab study (Registry number: ChiCTROOC-15006699) were recruited for the analysis. The serum 25(OH)D levels were classified into deficiency $(<20 \mathrm{ng} / \mathrm{mL})$, insufficiency $(20-29.9 \mathrm{ng} / \mathrm{mL})$ and sufficiency $(\geq 30 \mathrm{ng} / \mathrm{mL})$. A genetic risk score (GRS) was constructed based on four SNPs in the DBP gene that were previously reported as genetic determinants of $25(\mathrm{OH}) \mathrm{D}$ levels. Logistic regression models, multiple linear regression models and squared semi-partial correlation coefficients were conducted to evaluate the associations between SNP, haplotypes, GRS and 25(OH)D levels. The mean serum 25(OH)D was $20.19 \pm 5.44$ $\mathrm{ng} / \mathrm{mL}$. The ratios of $25(\mathrm{OH}) \mathrm{D}$ deficiency, insufficiency and sufficiency were $50.91 \%, 45.20 \%$ and $3.89 \%$, respectively. Significant associations of rs7041, rs4588, rs2282679 and rs705117 with serum 25(OH)D levels were found. Haplotype CG-harbored rs2282679 and rs705117 risk alleles increased the risk of $25(\mathrm{OH}) \mathrm{D}$ deficiency $(\mathrm{OR}=1.11,95 \% \mathrm{CI}$ : 1.08-1.15). The GRS was inversely associated with the serum $25(\mathrm{OH})$ D levels ( $=-0.008, p<0.001)$, and accounted for $3.3 \%$ variation in $25(\mathrm{OH}) \mathrm{D}$ levels. Compared with individuals in the lowest quarter, those in the fourth quarter of risk score had significantly increased risk for incident of $25(\mathrm{OH}) \mathrm{D}$ deficiency $(O R=2.27,95 \%$ CI: 1.79 2.89). In conclusion, rs7041, rs4588, rs2282679 and rs705117 in the DBP gene might be associated with the risk of $25(\mathrm{OH}) \mathrm{D}$ deficiency in the Chinese rural population.

3.128. PredMP: A Web Resource for Predicted Membrane Proteins in the Lipid Bilayer

S. Wang ${ }^{2, *}$, S. Fei ${ }^{1, *}$, Z. Wang ${ }^{3,4}$, Y. $\mathrm{Li}^{1}{ }^{1}$, X. Gao ${ }^{2}$ and F. Zhao ${ }^{1}$

1 Prospect Institute of Fatty Acids and Health, Qingdao University, Qingdao 266071, China

2 Computational Bioscience Research Center (CBRC), King Abdullah University of Science and Technology (KAUST), Thuwal 23955, Saudi Arabia

3 Department of Chemistry, Qingdao University, Qingdao 266071, China

4 James Franck Institute, University of Chicago, Chicago, IL 60637, USA

* Corresponding Authors

Experimental determination of membrane protein (MP) structures is challenging as they are often too large for nuclear magnetic resonance (NMR) and difficult to crystallize. There are only 510 non-redundant MPs with solved structures in the Protein Data Bank (PDB). To elucidate the MP structures, we developed a novel web resource http://52.87.130.56:3001/\#/proteinindex(PredMP), that delivers one-dimensional (1D) annotation of the membrane topology and secondary structure, twodimensional (2D) prediction of the contact/distance map, together with three-dimensional (3D) 
modeling of the MP structure in the lipid bilayer, for each MP from a given model organism. The precision of the constructed MP structures is leveraged by deep learning methods and modeling strategies. In particular, we (i) annotate 1D property via Deep Convolutional Neural Fields (DeepCNF) that model the complex sequence-structure relationship and interdependency between adjacent property labels; (ii) predict the 2D distance map through Deep Transfer Learning which learns the patterns as well as the complex relationship between distances and features from nonmembrane proteins; and (iii) model the 3D structure by feeding its predicted contacts and secondary structure to the Crystallography \& NMR System (CNS) suite combined with a membrane burial potential that is residue-specific and depth-dependent. PredMP contains more than 2200 multi-pass transmembrane proteins ( $<700$ residues) from Humans. These proteins are classified into a hierarchical organization of receptors, channels, transporters, enzymes and other drug targets according to molecular relationships and physiological functions. Among these MPs, we predict 1345-1871 targets including a few hundred new folds, which will facilitate the discovery of drugs targeted at MPs.

\subsection{A Genome-Wide Association Study in 79,366 European-Ancestry Subjects Informs the Genetic} Architecture of 25-Hydroxyvitamin D Levels

E. Hyppönen ${ }^{1}$, X. Jiang 2 ${ }^{\text {, P. F. O'Reilly }}{ }^{3}$, H. Aschard ${ }^{2}$, Y. H. Hsu ${ }^{4}$, B. Richards ${ }^{5}$, T. Spector ${ }^{6}$, J. Dupuis ${ }^{7}$, T. J. Wang ${ }^{8}$, P. Kraft ${ }^{2}$ and D. P. Kiel ${ }^{2}$, on behalf of the SUNLIGHT consortium.

1 Center for Population Health Research, University of South Australia, Australia

2 Harvard T.H. Chan School of Public Health, Boston, MA 02115, USA

3 King's College London, Institute of Psychiatry, UK

4 Institute for Aging Research, Boston, MA 02131, USA

5 Human Genetics, Epidemiology and Biostatistics, Montreal, QC H3A 1A2, Canada

King's College London, UK

Boston University School of Public Health, Boston, MA 02118, USA

8 Vanderbilt Heart and Vascular Institute, Nashville, 37232, USA

Vitamin D is a steroid hormone precursor that has established associations with a range of human traits and diseases. Previous GWAS of serum 25-hydroxyvitamin D concentrations have identified only four loci (GC, NADSYN1/DHCR7, CYP2R1, CYP24A1) harboring genome-wide significant variants. To better understand the genetic architecture underlying serum 25hydroxyvitamin $\mathrm{D}$, as well as to test for interactions between dietary vitamin $\mathrm{D}$ intake and genetic factors, we conducted the largest GWAS meta-analysis to date on this important vitamin. The first stage discovery meta-analysis consisted of 79,366 samples of European descent drawn from 31 studies (five times larger than our previously published GWAS) with in silico replication conducted in further 42,757 samples. In contrast to other GWAS with sample size increases of similar magnitude, this larger GWAS yielded only two additional loci harboring genome-wide significant variants $(p=$ $4.710^{-9}$ at rs8018720 in SEC23A, and $p=1.910^{-14}$ at rs10745742 in AMDHD1). The overall estimate of heritability of 25-hydroxyvitamin D serum concentrations attributable to GWAS common SNPs was $7.54 \%$, with statistically significant loci explaining $38 \%$ of this total (2.84\% out of $7.54 \%)$. Genomewide genotype-by-dietary vitamin D interaction analyses identified no significant interactions. Further investigation identified signal enrichment in immune and hematopoietic tissues, and clustering with autoimmune diseases in cell-type-specific analysis. These results suggest that common SNPs within only a small number of loci have notable influences on circulating 25hydroxyvitamin D concentrations. Genetic correlation and gene-enrichment analyses are in line with previous Mendelian Randomization studies supporting a putative causal association between vitamin $\mathrm{D}$ and autoimmune diseases.

\subsection{Defining a Pathway for Successful Implementation of Healthy Food Policy in a University} Environment

Jane Dancey, Claire Palermo, Vicki Ashton and Helen Truby 
Lack of access to healthier drinks and snack foods in vending machines can contribute to poor food choices. Universities provide a powerful setting for healthier choices to be promoted, especially to young adults. The Victorian Government's Achievement Program encourages workplaces to implement the Healthy Choices Guidelines (HCG) in food retail, catering and vending using the traffic light classification which stipulates that food and drinks are 50\% Green (best choice), 30\% Amber (choose carefully) and 20\% Red (limit). In 2017, Monash University commenced implementation of the Victorian Government's HCG commencing with vending-potentially impacting 70,000 students and 7500 staff. Senior leadership were required to approve the alteration to vending policy which included a competitive tender process. The tender required compliance with HCG plus standard financial and operational criteria. A photographic audit of 29 machines on one campus before implementation of HCG vending classified food and drinks as $64.8 \%$ Red, $13.7 \%$ Amber and $18.2 \%$ Green (3.3\% of product lines were empty). There are currently 35 new HCGcompliant vending machines on four campuses which offer $50 \%$ Green (an increase of $31.8 \%$ ), 30\% Amber (an increase of $16.3 \%$ ) and $20 \%$ Red (a decrease of $44.8 \%$ ) drinks and snacks. This process highlighted the necessity for senior leadership support and for compliance with HCG to be embedded into contractual arrangements. This has led to a large increase in the availability of Green drinks and snacks across a complex university environment. Implementation of HCG will now extend to catering and retail within this setting.

\title{
3.131. ATP2A1 rs3888190 SNP Is Associated with Increased Male Body Fat and Decreased Promoter Activity In Vitro
}

\section{J. T.-K. Wong ${ }^{1}$, S.-Y. Tan ${ }^{1}$, F.-T. Chew ${ }^{2}$ and Y.-H. Say ${ }^{1}$}

1 Department of Biomedical Science, Faculty of Science, Universiti Tunku Abdul Rahman (UTAR), Kampar, Perak, Malaysia

2 Department of Biological Science, Faculty of Science, National University of Singapore, Singapore, Singapore

rs3888190 SNP located upstream of ATP2A1 is an obesity GWAS loci and proxy SNP for SH2B1 rs7498665. We investigated the association of rs3888190 with obesity-related traits among 450 Malaysian adults (M/F: 214/236; Chinese/Indians: 425/25). Anthropometric measurements were taken and genotyping was performed by Taqman SNP Genotyping Assay. The promoter sequence of the ATP2A1 gene encompassing rs3888190 was cloned into promoterless firefly luciferase plasmid pGL4.10, co-transfected with Renilla luciferase plasmid pGL4.74 in HEK293 and T98G cells, and a dual luciferase reporter assay was performed. rs3888190 minor allele frequency for Chinese/Indians was 0.08/0.004. rs3888190 was not associated with BMI, waist circumference and total body fat (TBF) classes in overall subjects. However, stratified analysis showed that rs3888190 was significantly associated with mean TBF in Chinese males; those with AA genotype had significantly higher TBF compared with CC or CA genotypes (55.10\% vs. $16.93 \%$ or $17.03 \%$ ). rs 3888190 mutant A allele had up to 7 times lower relative luciferase activity compared with wild type G allele in HEK293 and T98G cells. PROMO 3.0 software predicted the loss of the p300/Elk-1 transcription factor binding site in A allele. In conclusion, rs3888190 A allele could be a functional risk allele for increased male adiposity by potentially decreasing ATP2A1 transcription.

\author{
3.132. The Combined Effect of FTO and MC4R Gene Polymorphisms on Obesity among Children and \\ Adolescents in Northern China \\ Y. Yang ${ }^{1}$, X. H. Gao ${ }^{2}$, X. J Tao ${ }^{1}$, Q. H. Gao ${ }^{1}$, Y. H. Zhang ${ }^{1}$ and J. J. Yang ${ }^{1}$ \\ 1 School of Public Health, Ningxia Medical University, Yinchuan, China \\ 2 Family Planning Service Center, Maternal and Child Health Hospital of Huangdao District, Qingdao, China
}


Background: The function of FTO and MC4R interactions in obesity is unclear among Chinese people, especially those in northern China. The aim of our study was to investigate whether their combined effects increased the risk of children and adolescents' obesity in northern China.

Methods: A total of 370 subjects (170 obese and 200 normal-weight) were enrolled using the random sampling method. FTO rs9939609, rs9935401 and MC4R rs12970134, rs17782313 interactions were analyzed by generalized multifactor dimensionality reduction (GMDR), and the logistic regression models were used to calculate the risk of the relation between genotypes and obesity.

Results: GMDR analysis showed a significant gene-gene interaction among FTO rs9939609/MC4R rs12970134/MC4R rs17782313, with a score of 10/10 for the cross-validation consistency and 9 for the sign test $(p=0.0107)$. A 2.453-fold increased risk for obesity was founded in individuals carrying the genotypes of FTO rs9939609 TA/AA, MC4R rs12970134 GA/AA and MC4R rs17782313 TC/CC (adjusted for age, sex and ethnicity; 95\% CI: 1.1205.374, $p=0.025$ ).

Conclusion: Our data support that the FTO rs9939609, MC4R rs12970134 and MC4R rs17782313 are strongly associated with obesity. Their combined effects were significant in child and adolescent obesity in northern China.

\title{
3.133. The Improvement in Diet Quality Following a Weight Gain Prevention Trial in Rural Dwelling
} Women

\author{
J. C. Martin ${ }^{1}$, L. J. Moran ${ }^{1}$, H. J. Teede ${ }^{1}$, C. B. Lombard ${ }^{2,+}$ and C. L. Harrison ${ }^{1,+}$ \\ 1 Monash Centre for Health Research and Implementation, School of Public Health and Preventative \\ Medicine, Monash University, Australia \\ 2 Department of Nutrition and Dietetics, Be Active Sleep Eat (BASE), Monash University, Australia \\ + These authors contributed equally
}

Reproductive-aged women are a high-risk group for obesity with subpopulations such as rural women at particular higher risk of obesity and longitudinal weight gain. While higher diet quality is associated with reduced weight gain, there is limited research exploring the role of optimizing diet quality in the setting of obesity prevention particularly in rural women. The study was a randomized controlled trial in rural women aged 18-50 years allocated to a 12 month low intensity selfmanagement lifestyle intervention $(n=114)$ or control group $(n=106)$ of standard lifestyle information. Outcomes were diet quality assessed by the Dietary Guideline Index, energy, macronutrient and micronutrient intake and predictors of change in diet quality. There was a significant association between the change in diet quality and change in weight in all participants on adjusted analysis $(-0.6695 \% \mathrm{CI}-1.2$ to $0.12 p=0.02)$. There was an improvement in diet quality (5.8 $95 \%$ CI 2.5 to $9.1 p=0.001)$, \% protein $(0.00995 \%$ CI 0.002 to $0.15 p=0.01)$ and glycemic index $(-1.2$ $95 \% \mathrm{CI}-2.1$ to $0.24 p=0.02)$ for the intervention compared to the control group. The intervention group was the only variable associated with the change in diet quality (5.8 95\% CI 2.5 to $9.1 p=0.001$ ). A low-intensity, self-management lifestyle intervention is effective in improving diet quality. Initiatives aimed at improving diet quality may contribute to the prevention of weight gain in young women in rural settings as a vulnerable, high-risk group for obesity development.

\subsection{GWAS Locus BDNF rs10767664 Is Associated with Obesity and Adiposity among Men in a Sample of Malaysian Han Chinese Diaspora}

\section{S.-Y.Choong ${ }^{1}$, F.-T. Chew ${ }^{1}$ and Y.-H. Say ${ }^{2}$}

1 Department of Biomedical Science, Faculty of Science, Universiti Tunku Abdul Rahman (UTAR), Kampar, Perak, Malaysia

2 Department of Biological Science, Faculty of Science, National University of Singapore, Singapore, Singapore

rs10767664, an intronic gene variant in the Brain-Derived Neurotrophic Factor (BDNF) gene, is an obesity genetic locus identified through various genome-wide association studies (GWAS). We investigated the association of rs10767664 with obesity-related traits among 419 Malaysian adults of the Han Chinese descent (M/F: 204/215). Anthropometric measurements were taken and genotyping 
was performed by Taqman SNP Genotyping Assay. The rs10767664 effect (A) allele frequency was 0.57. A significantly lower mean BMI $(p=0.04)$ and mean visceral fat to subcutaneous fat ratio $(p=$ $0.01)$ was found in men with $\mathrm{T}$ allele compared with those with A allele $(22.2 \pm 0.26$ vs. $23.0 \pm 0.25$ $\mathrm{kg} / \mathrm{m}^{2}$ and $4.29 \pm 1.80$ vs. $8.00 \pm 2.64$, respectively). Men with TT genotype or T allele had significantly lower risk of developing obesity (based on BMI class) compared with those carrying A allele (OR = $0.44 ; 95 \% \mathrm{CI}=0.21,0.93 ; p=0.03$ and $\mathrm{OR}=0.61 ; 95 \% \mathrm{CI}=0.37,1.00 ; p=0.047$, respectively). Similarly, men carrying the TT and TA genotype or $\mathrm{T}$ allele had significantly lower risk of having high overall adiposity (based on total body fat class) compared with those carrying A allele (OR $=0.49 ; 95 \% \mathrm{CI}=$ $0.25,0.94 ; p=0.04, \mathrm{OR}=0.40 ; 95 \% \mathrm{CI}=0.17,0.96 ; p=0.03$ and $\mathrm{OR}=0.61 ; 95 \% \mathrm{CI}=0.40,0.93 ; p=0.02$, respectively). In conclusion, the finding of rs10767664 as an obesity GWAS locus in several populations worldwide has been successfully replicated among men in a sample of Malaysian Han Chinese diaspora in this study.

\subsection{Preference for Food Literacy Program Participants to Build Connections through Facebook}

A. Devine, J. Rees, L. Costello, R. Sambell, C. Blom, C. De Backer and F. Mariani

1 School of Medical and Health Science, Edith Cowan University, Perth, Australia

2 The University of Antwerp, Antwerp, Belgium

3 The Good Foundation, Melbourne, Australia

Jamie's Ministry of Food Australia [JMoF] provides a mobile 7-week community-based food literacy program that teaches basic cooking skills to help people prepare simple, fresh, healthy food to combat increasing obesity and diet-related diseases. Food literacy outcomes may be further supported by health promotion strategies delivered through social media. In-depth interviews were conducted at three different locations in WA with 40 participants to determine if they valued opportunities to build social connections with other participants and which type of platform was preferred. Results indicated that an online forum, where participants could exchange recipes and discuss how to modify them, would be positively received. It was expected that a forum could also keep participants engaged and interested in healthy behaviours by reinforcing their newly acquired skills and building confidence about selecting and cooking healthy food for themselves and for family and friends. Most participants suggested that Facebook would provide an ideal communication channel for this type of forum and specifically a group page offered to participants along with the general community as a gateway to sharing information, providing support and building sustainable relationships. This led to the creation of the Foodie Network Facebook group. While the group is in its infancy, and it will take time to build a critical mass, new members are already joining, introducing themselves and starting conversations. Further research will determine if social connectedness and food literacy can be enhanced for those who participate in the Foodie Network. Broader health and wellness outcomes will also be investigated.

\subsection{Validity of Abdominal Fat Estimation Using Bioelectrical Impedance Analysis in Young Japanese Females}

\section{Kagawa and K. Uenishi}

Curtin University, Perth 6102, Australia

An accurate estimation of body composition is essential in health assessment. Bioelectrical impedance analysis (BIA) has been one of the common methods of body composition assessments. While there is a BIA device that is specifically designed to estimate percentage abdominal fat (\%AF) (AB-140, Tanita corp), its validity has not been fully reported. The present study was aimed to determine the validity of \%AF estimated from the BIA device using dual energy X-ray absorptiometry (DXA) as a reference method. A total of 45 Japanese young females (average age: 19.4 \pm 1.5 years) have completed anthropometry including umbilicus circumference, body composition assessments using DXA (Lunar Prodigy, GE Healthcare and enCore, v10.51.006, 2006), and AB-140. Average stature, body mass, and body mass index (BMI) were $159.0 \pm 5.2 \mathrm{~cm}, 52.9 \pm 5.8 \mathrm{~kg}$, and 20.9 
$\pm 2.0 \mathrm{~kg} / \mathrm{m}^{2}$ respectively. Estimated \%AF $(25.5 \pm 5.5 \%)$ was significantly smaller than percent trunk fat (\%TF) and percent android fat (\%Android) obtained from DXA (ranged between $28.3 \pm 6.0 \%$ and $31.1 \pm 7.8 \% ; p<0.01$ ) with only the exception of \%Android from the selected region of interest (\%Android_ROI) $(23.6 \pm 6.4 \% ; p<0.01)$. Minimum difference and bias were observed from \%Android_ROI with the limits of agreement of $-1.86 \pm 6.48 \%$. The current study showed a likelihood of underestimation when compared with variables automatically generated from DXA. However, it overestimated \%Android_ROI that may reflect visceral fat accumulation. Although further validation is required, the study may indicate the potential usefulness of this relatively new BIA device in assessment of \%AF.

\subsection{The Colour of Appetite: How Does Ambient Light Colour Affect Appetite?}

M. Kilburn ${ }^{1}$, J. Christian ${ }^{1}$, B. Bonetto ${ }^{2}$ and N. A. King ${ }^{1}$

1 Queensland University of Technology, Queensland, 4059, Australia

2 AgroparisTech, Paris 75231, France

Obesity is the second greatest contributor to the burden of disease in Australia with two out of three Australian adults overweight or obese. Overconsumption of food is a potent contributor to weight gain and obesity. A series of hedonic and homeostatic processes lead to increased drive to eat. Colour has long been associated with mood, and mood has also been shown to be associated with appetite. However, empirical evidence exploring whether directly manipulating light colour affects appetite is limited. This pilot study aimed to explore whether ambient light colour could moderate appetite. Fourteen lean, male university students (aged 1830 years) participated in three conditions on separate days. The conditions were blue light (B), pink light (P) and red light (R). Participants completed subjective appetite ratings via visual analogue scale (VAS) and Leeds-Oxford Food Preference Questionnaire (LFEPQ) measures immediately before and after exposure ( $3 \mathrm{~min})$ to the different light conditions. Preliminary analyses indicate that the colours exerted varying effects on subjective appetite. There was a trend for pink light to increase hunger by $5 \%$, whereas blue and red light suppressed hunger by $5 \%$. Although changes were relatively small, there was a trend for both blue and red light to exert a different effect on Liking and Wanting compared to pink light. This pilot study provides preliminary evidence that pink light exerts differential effects on appetite compared with blue and red light.

\subsection{A Novel Prescription Pedometer-Assisted Walking Intervention and Weight Management for Chinese Working Population \\ C. Chang ${ }^{1}, \mathrm{Y} . \mathrm{Yu}^{1}{ }^{1}, \mathrm{Y} . \mathrm{Lv}^{2}, \mathrm{X} . \mathrm{Zhhang}^{1}$ and L. Xie ${ }^{1}$ \\ 1 Third Hospital of Peking University, Beijing, China \\ 2 Chinese Center of Diseases Control and Provention, China}

Brisk walking has been confirmed to benefit weight management. The effective delivery of health behavior interventions with android wear remains to be investigated in the working population. We conducted a 3-month open, self-controlled clinical trial among 802 participants aged 20 to 65 years to assess the feasibility and effectiveness of a lifestyle weight management program among the working population, using an information technology (IT)-supported platform and wearable prescription pedometer. Each personalizing exercise prescription was loaded in the special prescription pedometer carried by the participant to instruct and monitor walking. After 3 months intervention, 718 participants completed the exercise program with an exercise compliance rate of $89.5 \%$. Weight or body mass index decreased by $2.2 \%$ among 305 overweight and obese participants, with a reduction of $3.2 \%$ in waist circumference and $1.9 \%$ in body fat percentage $(p<0.05)$. About $68.4 \%$ of the overweight and obese participants experienced weight loss after intervention, with an average decrease of $3.5 \%$ ( $2.8 \mathrm{~kg}, p<0.001)$, and $20.9 \%$ of them achieved weight loss $\geq 5 \%$. For 346 normal-weight individuals, weight (or BMI) and body fat percentage decreased by $1 \%$ and $2.8 \%$ ( $p<$ $0.05)$, respectively. A weight gain of $1.0 \%$ was found in all underweight participants $(p<0.05)$. 
Additionally, the detection rate of hypertension, hyperglycemia, and hyperlipaemia was significantly lower and the lifestyle of all participants was significantly better than before. In conclusion, the ITassisted and prescription pedometer-based lifestyle intervention was practicable and effective to manage weight and control the risks of weight-related chronic diseases for working groups.

\subsection{Intermittent Fast Diet: Changes in Weight, Quality of Life and Eating Behaviours in Adolescents with Obesity}

H. Jebeile 1,2, M. L. Gow 1,2, N. Lister 1,2, K. Chisholm 2, A. Grunseit ${ }^{2}$, S. Alexander 2, C. T. Cowell 2, L. A. Baur ${ }^{1,2}$ and S. P. Garnett 1,2,5

1 The University of Sydney, Discipline of Child and Adolescent Health, Sydney 2000, Australia

2 The Children's Hospital at Westmead, Sydney 2000, Australia

Intermittent fast diets (IFD) in adults are as effective as continuous energy restriction. We are investigating the effectiveness and acceptability of an IFD in adolescents with obesity. Weeks 1-12: participants follow an IFD including a Very Low Energy Diet (VLED) 3 days/week (500-600 kcal/day) and a standard healthy diet 4 days/week. Weeks 13-26: participants choose to continue with 1-3 days of VLED/week or follow a standard healthy diet. Outcomes at 0,12 and 26 weeks are weight, health related quality of life (HRQOL, PedsQL) and eating behaviours (DEBQ-child). 30 participants, aged 12-17 years (mean[SD] $14.5 \mathrm{yrs}[1.4]$, female $\mathrm{n}=25$ ) with a median BMI $34.9 \mathrm{~kg} / \mathrm{m}^{2}$ (range: $27.7-52.4$ ), were recruited and to date, 19 have completed the 26 week protocol. The study will finish in August 2017. For this abstract, we report outcomes for participants with complete data. Compared with baseline, both body weight and BMI were significantly reduced at 12 weeks (mean[SD], $\mathrm{n}=20,-3.5$ $\mathrm{kg}$ [3.7] $\left.p=0.001,-1.4 \mathrm{~kg} / \mathrm{m}^{2}[1.3] p<0.0001\right)$, not maintained at 26 weeks $(\mathrm{n}=18,-2.1 \mathrm{~kg}[7.9],-0.9$ $\left.\mathrm{kg} / \mathrm{m}^{2}[2.6]\right)$. At baseline $(\mathrm{n}=13)$ mean [SD] scores for total HRQOL, restrained, emotional and external eating were $75.75[3.96], 2.38[0.43], 1.71[0.15]$ and 2.10[0.11] respectively. Dietary restraint significantly increased from baseline to 26 weeks 2.66[0.08], $p=0.043$ ) with no change in emotional or external eating and HRQOL. Intermittent fast diets appear to be safe and may be an effective dietary intervention in adolescents with obesity. A randomised controlled trial is required to compare IFD with continuous energy restriction.

Funded by the Financial Markets Foundation for Children and Heart Foundation of Australia Vanguard Grant.

\subsection{Anti-Obesity and Anti-Diabetic Effects of Nitrate and Nitrite}

Asghar Ghasemi and Sajad Jeddi

Endocrine Physiology Research Center, Research Institute for Endocrine Sciences, Shahid Beheshti University of Medical Sciences. Tehran, Iran

The prevalence of obesity is increasing worldwide and type 2 diabetes, to date, is the most devastating complication of obesity. Decreased nitric oxide bioavailability is a feature of obesity and diabetes that links these two pathologies. Nitric oxide is synthesized both by nitric oxide synthase enzymes from L-arginine and nitric oxide synthase independent from nitrate/nitrite. Nitric oxide production from nitrate/nitrite could potentially be used for nutrition-based therapy in obesity and diabetes. Nitric oxide deficiency also contributes to pathogeneses of cardiovascular disease and hypertension, which are associated with obesity and diabetes. This review focuses on the anti-obesity and anti-diabetic effects of the nitrate-nitrite-nitric oxide pathway. In addition to increasing nitric oxide production, nitrate and nitrite reduce oxidative stress, increase adipose tissue browning, have favorable effects on nitric oxide synthase expression, and increase insulin secretion, all effects that are potentially promising for the management of obesity and diabetes. Based on current data, it could be suggested that amplifying the nitrate-nitrite-nitric oxide pathway is a diet-based strategy for increasing nitric oxide bioavailability and the management of these two interlinked conditions. Adding nitrate-nitrite to drugs that are currently used for managing diabetes (e.g., metformin) and possibly anti-obesity drugs may also enhance their efficacy. 


\title{
3.141. Efficacy of A Technology-Based 12-Week Weight Loss Intervention Program, 'DHC DIET AWARD
} $2015^{\prime}$

S. Kamohara, M. Onishi, Y. Imataka, N. Hasegawa and H. Seki

Affiliation not provided.

Background: Clinic-based liquid meal replacement programs are widely used for weight management. However, little is known about the safety and efficacy of a technology-based intervention for weight-loss. The aim of this study is to evaluate the feasibility and efficacy of a telephone- and/or web-based 12-week weight loss intervention with a commercial liquid meal replacement program, 'DHC Diet Award 2015'. Methods: Japanese obese subjects, otherwise healthy, participated in the program, using telephone and/or a personalized web site to receive individual dietary counseling from medical professionals. Commercial liquid meal replacements, 'DHC Protein Diet' series, were consumed once or twice daily instead of regular meals. A typical product contains $20.0 \mathrm{~g}$ of protein, one-third of the RDA of vitamins and minerals, $7.5 \mathrm{~g}$ of dietary fiber in 1 bag gross weight $50 \mathrm{~g}$ or $178 \mathrm{kcal}$. A genetic test to detect SNPs in $\beta 3 \mathrm{AR}, \beta 2 \mathrm{AR}$ and UCP1 related to susceptibility to obesity was also used with life-style questionnaires. Their body compositions were assessed before and after a 12-week weight-loss program. Results: After 12 weeks, 366 participants (96.8\%, male 55, female 311) out of 378 who completed the program, showed decreased body weight compared with baseline. Of the other participants, 11 showed increased body weight and one had no change. A significant decrease of the BMI was observed (male; from 28.4 to 24.3 , female; from 27.2 to 23.4). Conclusion: Our results demonstrated that a technology-based intervention, combined with commercial meal replacements is effective for reducing body weight in obese subjects.

\subsection{Protocol: Optimal Dietary Macronutrient Distribution in China (ODMDC) Trial}

\author{
Y. Wan ${ }^{1}$, F. Wang ${ }^{1}$, J. Yuan ${ }^{2}$, D. Li ${ }^{1,3}$ \\ 1 Department of Food Science and Nutrition, Zhejiang University, Hangzhou, China \\ 2 No. 1 Department of Nutrition, Chinese PLA General Hospital, Beijing, China \\ 3 Institute of Nutrition and Health, Qingdao University, Qingdao, China
}

Findings from observational studies in China showed that the increased dietary fat consumption might be a cause for considerable concern, leading to an obesity epidemic. However, some cohort studies reported that the carbohydrate intake, especially white rice, is associated with risk of obesity, type 2 diabetes and coronary heart disease in China. The aim of our study is to determine whether the traditional lower fat, higher carbohydrate diet or the western higher fat, lower carbohydrate diet is more effective for obesity prevention and related cardiometabolic profile in modern China. Optimal Dietary Macronutrient Distribution in China (ODMDC) Trial is a 6-month, multi-center, three-arm controlled feeding study. Based on the macronutrient transition in the past 30 years in China, we designed three isocaloric diets with a spectrum of fat and carbohydrate intake, but the same protein contents. Percentages of energy derived from fat, carbohydrate, and protein are $20 \%$, $66 \%$ and $14 \%$ in the first diet group; $30 \%, 56 \%$ and $14 \%$ in the second diet group; $40 \%, 46 \%$ and $14 \%$ in the third diet group, respectively. Participants will be provided with all of their food and most beverages for 6 months. The study population is expected to be 300 healthy non-obese adults aged 18 to 35 years. The primary outcome variable is body weight and cardiometabolic risk factors. Results from the current trial will have important implications in nutrition recommendations for the prevention of obesity and related cardiometabolic disturbances among healthy non-obese Chinese.

\subsection{Coffee Residue Attenuates Diet-Induced Metabolic Syndrome in Rats}

N. S. Bhandarkar, S. K. Panchal and L. Brown

Functional Foods Research Group, University of Southern Queensland, Toowoomba, QLD 4350, Australia

Coffee is ubiquitous as a beverage and many studies have investigated potential health benefits and adverse effects. However, only few studies have investigated possible health benefits of the 
residue from coffee brewing containing caffeine, chlorogenic acid, trigonelline, soluble and insoluble fibre, micronutrients and melanoidins. Coffee residue has been reported for its anti-oxidant properties. Fibres from coffee residue have shown positive modulation of gut activity as prebiotics. In this study, male Wistar rats (8-9 weeks old, $340 \pm 5 \mathrm{~g}, \mathrm{n}=48$ ) were given a diet high in simple sugars such as fructose and sucrose together with increased long-chain saturated and trans fatty acids for 16 weeks to induce metabolic, cardiovascular and liver changes characteristic of human metabolic syndrome. Rat food was supplemented with 5\% coffee residue for the last 8 weeks of the protocol. The high-carbohydrate, high-fat diet induced hypertension, dyslipidaemia, central obesity, impaired glucose tolerance and non-alcoholic fatty liver disease. In these rats, coffee residue reduced visceral fat deposition without affecting food and water intake, reversed increased systolic blood pressure and attenuated increased left ventricular diastolic stiffness and reduced infiltration of inflammatory cells and collagen deposition in the heart. Coffee residue treatment decreased liver inflammation, fat deposition in the liver, and plasma concentrations of triglycerides and non-esterified fatty acids. These results suggest that constituents of coffee residue improved obesity-related cardiovascular, liver and metabolic changes in diet-induced obese rats, possibly by suppressing inflammation.

\subsection{Change in Fruit and Vegetable Consumption Following the Cook Chill Chat Program}

\section{Mutua ${ }^{1}$, J. C. Russell ${ }^{1}$, S. Wright-Pedersen ${ }^{2}$ and D. Condon-Paoloni ${ }^{1}$}

1 School of Health and Society, University of Wollongong, Wollongong, Australia

2 Healthy Cities Illawarra, Fairy Meadow, NSW, Australia

The Cook Chill Chat (CCC) program is a six-week social inclusion cooking program run by Healthy People Illawarra which aims to increase social connectedness, improve physical and mental wellbeing, nutrition knowledge and cooking skills within the Illawarra community. The aim of this study was to assess changes in self efficacy, dietary habits and food skills following the six week CCC program. The evaluation was a pre-post study design, where quantitative data was collected from 116 participants using structured questionnaires before and after the program. The baseline and follow-up questionnaire assessed the change in participants' social connectedness, dietary habits, self-efficacy, food security and food skills. Statistical analysis of the quantitative data was conducted using McNemar, paired $t$ and chi squared tests. There was a significant increase in mean daily intake of vegetables from 2.72 to $3.65(p=0.00)$ and significant decrease in mean weekly serves of sugar sweetened beverages and mean monthly serves of take away from 6.11 to $3.48(p=0.02)$ and 3.64 to $2.60(p=0.04)$ respectively. However, there was no significant change in daily fruit intake (from 2.07 to $2.22, p=0.15)$. The initial evaluation showed that the CCC program was successful in increasing food skills and self-efficacy of the participants which consequently led to an increase in vegetable intake and decrease in takeaway and sugar sweetened beverage consumption. The next steps will be to assess these changes, as well as change in social connectedness and food security, at the six month follow up.

\subsection{Luteolin Prevents Obesity via Promoting Browning and Thermogenesis in Metabolic Organs of Mice with Diet Induced Obesity}

\section{E. Y. Kwon, S. Y. Kim, E. J. Do and M. S. Choi}

Center for Food and Nutritional Genomics Research, Department of Food Science and Nutrition, Kyungpook National University, Daegu, Korea

Obesity is a medical condition in which excess body fat has accumulated to the extent that it may have an adverse effect on health, and its complications are potential and important causes of metabolic syndrome. Luteolin is a dietary flavonoid with anti-inflammatory, anti-cancer and antiobesity properties. However, little is known about the detailed mechanism associated with the effect of luteolin on browning and thermogenesis in diet-induced obesity. The aim of the present study was to reveal the browning and thermogenesis effect of luteolin in metabolic organs such as muscle, white and brown adipose tissue of diet-induced obesity mice suing "transcriptomics" tool. Thirty-nine male 
C57BL/6J mice (4-week-old) were randomly divided into three groups and were fed normal diet, high-fat diet (HFD, 20\% fat) and HFD + 0.005\% ( $w / w)$ luteolin for 16 weeks. Luteolin decreased body fat mass, while it increased energy expenditure. In skeletal muscle, luteolin increased oxidative capacity and fatty acid utilization by elevating the enayme activities of citrate synthase (CS), cytochrome $\mathrm{C}$ oxidase (COX) and $\beta$-hydroxyacyl CoA dehydrogenase (HOAD) and UCP3 gene expression compared to HFD. Moreover, our microarray results of muscle and brown and brown and white adipose tissue revealed that down-regulated gene expressions associated with the TCA cycle by HFD were up-regulated by luteolin in white adipose and muscle tissue. Luteolin treatment directly elevated thermogenic gene expression in brown and epididymal adipose tissue. Taken together, our results indicate that luteolin increased energy expenditure and altered the muscle, white and brown adipocyte browning and thermogenesis metabolizing factors, thereby preventing obesity.

\subsection{University Healthier Choice Catering Guidelines: Development, Implementation and Social Marketing}

\section{S. R. Partridge ${ }^{1,+}$, N. Dergham ${ }^{1,+}$, L. Kairey ${ }^{1}$, E. Howse ${ }^{1}$, K. A. Sim ${ }^{2}$ and M. Allman-Farinelli ${ }^{1}$}

1 The University of Sydney, Nutrition and Dietetics Group, School of Life and Environmental Science, Charles Perkins Centre, Sydney, NSW 2006, Australia

2 The University of Sydney, The Boden Institute of Obesity, Nutrition, Exercise \& Eating Disorders, Charles Perkins Centre, Sydney, NSW 2006, Australia

+ These authors contributed equally.

Poor diet is a leading cause of the non-communicable disease (NCD) burden globally. The increasing prevalence of NCD has the potential to result in significant losses in workforce capacity and productivity. As employees spend almost half their waking hours at work, workplace health promotion initiatives and food environment modification are indicated measures to address this issue. This study aimed to (i) develop healthy catering guidelines for a large university; and (ii) develop a social marketing campaign to promote the utilization of the guidelines by staff members and catering companies. Firstly, an online survey and semi-structured telephone interviews investigated staff attitudes regarding catered events. This formative research informed the Healthier Choices Catering Guidelines (HCCG) that were then designed to adhere with national dietary guidelines. To test the feasibility of producing HCCG compliant menus, semi-structured telephone interviews with catering companies investigated factors affecting their ability to produce compliant menus. Finally, a social marketing campaign was developed to promote utilization of the guidelines. Survey results found nearly all staff $(99 \%, 333 / 338)$ agree it is important to have healthy food and drink options available at meetings and functions. Further, interviews with staff members responsible for catering events $(n=7)$, found orders were influenced by guests attending, meal occasion, cost, dietary requirements, gender, age and catering company menu and services offered. Interviews with cater companies $(n=5)$ revealed they were content producing HCCG compliant menus. Research is underway to determine the impact of the social marketing campaign for increasing awareness and utilization of the HCCG.

\subsection{Understanding Ethnic Differences in Portion Sizes in New Zealand Adults from the 2008/09 National Nutrition Survey}

A. R. Molenaar ${ }^{1}$, M. L. McIntyre ${ }^{2}$, H. Carr ${ }^{2}$, M. Turley ${ }^{2}$ and T. A. McCaffrey ${ }^{1}$

1 Department of Nutrition, Dietetics and Food, Monash University, Melbourne, Australia

2 New Zealand Ministry of Health, Wellington, New Zealand

National food consumption surveys provide valuable information on ethnic differences in eating habits that can be used to inform the development of food based dietary guidelines. The objective of this study was to examine differences in portion size (PS) and frequency of consumption of 33 food groups from the 2008/09 New Zealand Adult Nutrition Survey (NZANS). Data from one 24-h dietary recall were used to calculate PS for 33 food groups, and examine differences by anthropometric and demographic factors. The survey included 4721 adults 15 years or older (56.2\% females), $22 \%$ with a prioritised ethnicity of Māori, 14.8\% Pacific Islander and 63.1\% New Zealand European or Other 
(NZEO). Over $40 \%$ of participants were aged $19-50$ years with $22.6 \% \geq 71$ years, $33.5 \%$ were classified as overweight and $32.0 \%$ as obese. The top 10 most frequently consumed food groups were nonalcoholic beverages, milk, vegetables, sugar/sweets, fruit, bread, fat spreads, savoury sauces, starchy vegetables and grains and pasta, with similarities across ethnic groups and BMI categories. PS were significantly smaller $(p<0.0167)$ for NZEO participants for $>75 \%$ food groups relative to Māori ( $\uparrow$ PS 25/33) and Pacific Islanders ( $\uparrow$ PS 27/33). Māori participants consumed significantly smaller PS than Pacific Islanders for $8 / 33$ food groups $(p<0.0167)$. Males consumed significantly $(p<0.05)$ larger PS than females for $27 / 33$ foods groups. While the most frequently consumed food groups are similar across the population, differences exist in PS between Māori and Pacific people compared with NZEO. This information may assist with the development of appropriate public health messaging around PS.

\subsection{A Systematic Review of Estimation Errors for Portion Size Estimation Aids during Dietary Recall in Adults}

A. Molenaar ${ }^{1}$, M. Rollo ${ }^{2}$, N. de Vlieger ${ }^{2}$, M. Weltert ${ }^{2}$, C. Collins ${ }^{2}$, T. Bucher ${ }^{2}$ and T. McCaffrey ${ }^{1}$

1 Department of Nutrition, Dietetics and Food, Monash University, Melbourne, Australia

2 School of Health Sciences, Faculty of Health and Medicine \& Priority Research Centre for Physical Activity and Nutrition, The University of Newcastle, Newcastle, Australia

Portion size estimation aids (PSEAs) are used to assist with quantification of portions size (PS) during dietary recall. There is a need to systematically examine PS estimation error (PSEE) using PSEAs for different foods. Eight electronic databases were systematically searched. Inclusion criteria were English language, participants aged $\geq 17$ years, recall estimation of PS using a PSEA and PSEE reported. Fifteen studies were eligible for inclusion. The majority (n12) of studies were published between 1992 and 2006. Overall, 60.9\% of participants were female. Food photographs were the most commonly used PSEA (n10) with between one and 11 pictures of varying PSs. Other PSEAs included food diagrams (n2), ruler (n4), adjustable wedge (n2), size grid (n1), bean bags (n3), 3D models (n1), computer based images (n1) and household measures (n1). Participants were either asked to estimate the PS immediately after exposure to food (n7) or between one to four days post-exposure (n7) or both. PSEAs were used to recall either consumption of between 3 and 28 foods following a meal (n10) or presentation of between 1 and 9 food types without consumption (n5). The type of PSEA used with the same food was not consistent between studies. There was a large variation in PSEE ( $0 \%$ to $242.9 \%$ ) with no type of PSEA or food consistently resulting in lower PSEE, which may be due to the heterogeneity of studies. There is a need for more studies with consistent study designs to allow for further comparison of PSEE using PSEAs during dietary recall.

\subsection{A Comparison of Temporal Distribution of Energy and Macronutrient Intakes between Morning and Evening Exercise}

P. G. Brooker ${ }^{1}$, S. R. Gomersall ${ }^{2}$, N. A. King ${ }^{3}$ and M. D. Leveritt ${ }^{1}$

1 School of Human Movement and Nutrition Sciences, The University of Queensland, St. Lucia, Queensland, Australia

2 School of Health \& Rehabilitation Sciences, The University of Queensland, St. Lucia, Queensland, Australia

3 School of Exercise \& Nutrition Sciences, Queensland University of Technology, Kelvin Grove, Queensland, Australia

The distribution of energy intake (EI) could play a role in the effectiveness of weight loss interventions. Exercise can alter EI via changes in appetite and food preferences, but the impact of the time-of-day of exercise remains unknown. The purpose of this study was to investigate how individuals distribute their EI in response to morning or evening exercise. Forty-one inactive, overweight adults were randomised into one of two 12-week exercise groups: morning exercise (AMEx); or evening exercise (PMEx). Participants were asked to recall 24-h food and beverage consumption on two training days at the mid-point of the exercise intervention, using a five-step multiple-pass recall. Absolute daily energy and macronutrient intakes, and temporal distribution of 
energy and macronutrients were calculated. Macronutrient intakes were also calculated as a proportion of total daily EI. There were no differences in absolute amounts of total daily EI, carbohydrate or fat between groups. PMEx consumed more protein than AMEx $(p<0.05)$. Relative to total energy, AMEx consumed more of their energy from fat than PMEx $(p<0.05)$. There were no differences in the proportion of carbohydrate or protein consumption between groups. When considering the temporal distribution, there was a trend for the AMEx groups' EI to be higher in the lunch period compared to the PMEx group, whose EI was higher in the dinner period. This study supports previous work that exercise can moderate appetite. Since eating later in the day is associated with increased EI and weight gain, exercise timing may play a role in weight management.

\subsection{Energy Intake from Snacking in Australian Adults from the Australian National Nutrition and} Physical Activity Survey 2011-2013

\section{J. A Barbour ${ }^{1,2}$, A. M. Coates ${ }^{1}$ and K. M. Dickinson ${ }^{2}$}

1 Alliance in Research in Exercise, Nutrition and Activity, University of South Australia, Adelaide, SA 5001, Australia

2 Discipline of Nutrition and Dietetics, College of Nursing and Health Sciences, Flinders University, Bedford Park, SA 5042, Australia

Snacks can benefit intake by contributing nutrients to the diet; in contrast, the growing trend in "grazing" is associated with an increase in obesity. This study determined energy intake from snack foods consumed by adults (19+ years) using $24 \mathrm{~h}$ recall data from the 2011-2012 Australian National Nutrition and Physical Activity Survey. A snack was defined as any food or beverage (excluding alcohol) consumed between meals or a self-identified "snack" eating occasion. The relationship between body mass index (BMI) and percentage energy from snacks was assessed. Of the total number of subjects $(\mathrm{N}=9341), 96.5 \%$ consumed at least one food or beverage "snack" item. Males consumed $31 \%$ of their total energy intake as snacks and females $27 \%$. Snacks consisted of savoury non-core items, mainly processed ( $2 \%$ of total energy), sweet non-core items, mainly sweetened beverages and biscuits (13\%) and core food items, mainly dairy, breads and cereals (12\%). Of the total energy, $14 \%$ was consumed as beverages (milk, juice, tea, coffee and soft drinks). The highest proportion of energy from snacks was consumed by males aged $19-50$ years (35\%) and the lowest by males over 70 years (21\%). Spearman's correlation showed an inverse association between BMI and percentage of total energy from snacks $(\mathrm{r}=-0.26, p=0.022)$. These findings suggest that despite a high proportion of energy consumed via snacks and sweetened beverages, snacking does not contribute to increase in body weight. Core snack foods may be useful in providing additional nutrients in the diet of adults.

\subsection{Omega-3 PUFA for Reduction of Liver Fat: The Evidence to Date}

\section{H. M. Parker ${ }^{1}$, N. A. Johnson ${ }^{1}$, C. A. Burdon ${ }^{2}$, J. S. Cohn ${ }^{3}$, H. T. O'Connor ${ }^{1}$ and J. George ${ }^{4}$}

1 The University of Sydney, Sydney 2000 Australia

2 The University of Wollongong, Wollongong 2500, Australia

3 The Heart Research Institute, Sydney 2000, Australia.

4 Storr Liver Unit, Westmead Millennium Institute, Sydney 2000, Australia

Background: Non-alcoholic fatty liver disease (NAFLD) is associated with obesity, and is an independent predictor for cardiovascular disease. Yet the only accepted therapy remains weight loss via diet and exercise. Omega-3 polyunsaturated fatty acids (PUFA) represent a promising treatment, and consequently a fast-growing area of research. This systematic review aimed to collate the evidence to date for the effect of omega-3 PUFA on liver fat in adult humans. Methods: Databases were searched for studies of dietary omega-3 PUFA supplementation in human adults for the treatment of raised liver fat with or without hepatic inflammation (i.e., studies in NAFLD and nonalcoholic steatohepatitis; NASH) published since 2011. The search results were added to those from an original search conducted in 2012. Studies were included if outcome measures included change in liver steatosis. Results: The search netted 3016 non-duplicated articles, in addition to the original 
23,592 articles. Sixteen studies used PUFA for treatment of NAFLD/NASH in adults. Of these, 12 randomised and three non-randomised trials included liver steatosis as an outcome measure. Dose ranged from $0.83 \mathrm{~g}$ to $50 \mathrm{~mL}$ per day of omega-3 PUFA. Although NASH Activity Score did not change, three of five randomised studies in NASH, and five randomised studies in NAFLD demonstrated significant reduction of liver fat with PUFA. Conclusion: Omega-3 remains a promising agent for liver fat reduction in NAFLD and NASH, however further research is required to quantify the expected benefit, factors that influence benefit, and to establish a dose-response for this therapy.

3.152. Sugar-Sweetened Beverages Intake and Risk of Obesity and Weight Gain in Children and Adolescents: A Meta-Analysis of Studies in the West and Asia

Caicui Ding, Haijun Guo, Chao Song, Fan Yuan, Weiyan Gong and Ailing Liu

National Institute for Nutrition and Health, Chinese Center for Disease Control and Prevention, Beijing 100050, China

Background: To examine the association between sugar-sweetened beverages (SSBs) consumption and risk of obesity and weight gain in children and adolescents. Methods: PubMed, Cochrane Library, ELSEVIER Science Direct, Ovid, ProQuest, SpringerLink database, CNKI and Wanfang databases were searched. We included studies from January 1997 to July 2016 without language restrictions. Summary statistics (Odds Ratios, ORs) were used to convey the association of SSBs and obesity. The overall estimates and CIs for the association of the difference $(\Delta)$ in SSBs consumption with $\Delta$ body mass index (BMI; in $\mathrm{kg} / \mathrm{m}^{2}$ ) were produced to express the SSBs and weight gain. Results: A total of 13 studies met the inclusion criteria, 7 for the analysis of SSBs and obesity (n $=15,393)$, and 7 for weight gain $(n=17,227)$ (1 study was included in both). Overall, SSBs intake can significantly increase the risk of obesity in children and adolescents (OR: 1.41; 95\% CI: 1.12, 1.76; $p=$ 0.003). Subgroup analysis by study location suggested that SSBs were associated with a bigger increase of obesity risk in Asian children (OR: 1.742; 95\% CI: 1.495, 2.030) than in westerners (OR: 1.265; 95\% CI: 1.120, 1.428). There was a positive association between SSBs and BMI: one daily 12-oz serving increment of SSBs was associated with a 0.034 (95\% CI: -0.028, 0.097)-unit increase in BMI in children and adolescents. Conclusion: SSBs intake is positively and significantly associated with the development of obesity. The association between SSBs and body weight gain in children was positive but not statistically significant.

\subsection{Genetic and Environmental Relationships between Eating Behavior and Symptoms of Anxiety and} Depression

K. Lee

Department of Family Medicine, Busan Paik Hospital, Inje University College of Medicine, Busan, Korea

(1) Background: To assess gender-specific genetic and environmental correlations between depressive and anxiety symptoms, and concurrent and follow-up eating behavior in Korean twins and their family members; (2) Methods: Center for Epidemiological Studies Depression Scale and State-Trait Anxiety Inventory were used to measure depressive and anxiety symptoms in subjects. To assess concurrent and follow-up relationships of the symptoms with eating behavior domains (restrained, emotional, and external eating), the Dutch Eating Behavior Questionnaire was administered to 2359 subjects at baseline (men, $48.5 \% ; 42.0 \pm 12.7$ years old; monozygotic twins, $33.7 \%$ ) and to 1169 subjects at follow-up (men, $45.9 \% ; 44.9 \pm 11.6$ years old; monozygotic twins, 41.0\%). A mixed linear model and bivariate analysis were applied; (3) Results: After adjusting for age, twin and family effects, income, education, smoking status, alcohol use, exercise, and body mass index, depressive and anxiety symptoms were positively associated with concurrent and follow-up emotional and external eating, but not with restrained eating. The effect size of association with emotional eating increased in men over time, but decreased in women. Common genetic and environmental correlations showed a difference between genders, and their strength changed with 
time. Nevertheless, common genetic correlations were found between depressive and anxiety symptoms, and concurrent emotional eating, in both genders. There were common environmental correlations between anxiety symptoms, and concurrent restrained and emotional as well as followup emotional eating, in both genders; and (4) Conclusion: There are similarities and differences in genetic and environmental relationships between depressive and anxiety symptoms and eating behaviors, based on gender and time of assessment.

\subsection{Impaired Regulation of Hypothalamic CD36 and Neuropeptides in Diet-Induced Obese Rats}

Xiaoyi Wang, Yan Ma and Nianhong Yang *

Department of Nutrition and Food Hygiene, Hubei Key Laboratory of Food Nutrition and Safety, Ministry of Education (MOE) Key Lab of Environment and Health, School of Public Health, Tongji Medical College,

Huazhong University of Science and Technology, China

* Corresponding author.

We aimed to measure the expression of a hypothalamic fatty acid receptor cluster of differentiation 36(CD36) and selected appetite neuropeptides, and compare the possible difference in rats with different susceptibility to high fat diet-induced obesity. Diet-induced obese (DIO) and dietinduced obese resistant (DIR) rats were developed by 5 weeks of ad libitum high-fat diet feeding. Food intakes were measured each day and body weight was measured each week. At the end of the experience, rats were sacrificed in either fasting or refeeding states. Hypothalamic CD36 and neuropeptides expressions were measured using Western blotting and real-time PCR. Biochemical indicators such as serum glucose, insulin, total cholesterol and triglycerides, were determined by corresponding kits. Both the average food intake and body weight of DIO rats were significantly higher than those of the DIR rats. Under fasting condition, protein expressions of CD36, neuropeptide Y(NPY) and agouti-related protein (AgRP) were higher in DIO rats than DIR rats. In DIR rats, hypothalamic NPYAgRP and CD36 protein levels were down-regulated significantly after the refeeding situation compared with in the fasting condition. However, these regulations were attenuated in DIO rats. In conclusion, DIO rats had a higher fasting protein expression of hypothalamic CD36 and NPY/AgRP than DIR rats, and the impaired downregulation of the above protein levels in response to high fat diet feeding in DIO rats may be related to the mechanism of overeating and susceptibility of obesity induced by high fat diet.

\subsection{Eicosapentaenoic Acid and Oleic Acid Protect Cultured Hypothalamic Neurons against Palmitic Acid Induced Lipotoxicity via Separate Mechanisms}

D. Sergi, P. Kubitz, A. Morris and L. M. Williams

Obesity and Food Choice Theme, Rowett Institute, University of Aberdeen, Aberdeen Scotland

Lipotoxicity is emerging as a key mediator of hypothalamic dysfunction leading to hypothalamic inflammation, ER stress and resistance to anorexigenic hormones. We have previously shown that oleic acid (OA) and eicosapentaenoic acid (EPA) counteract palmitic acid (PA)-induced ceramide accumulation in mHypoE-N42 hypothalamic cells, but the mechanisms underlying these effects are unclear. Thus, we investigated how OA and EPA exert their protective effects against PAinduced ceramide lipotoxicity. mHypoE-N42 cells were challenged with $200 \mathrm{M}$ PA in the presence and absence of either OA or EPA $(125 \mathrm{M})$. Mitochondria were stained using MitoTracker Red and the expression of Mitofusin 2 ( $m f n 2$ ) was assessed by Real-Time PCR. Triglycerides were stained with Oil Red O. Image analysis of mitochondria and lipid droplets was carried out using ImageJ. Both OA and EPA, either alone $(p<0.001)$ or in combination with PA $(p<0.05)$, increased total cellular mitochondria content relative to PA alone, with EPA, but not OA, being able to increase mitochondrial fusion either alone $(p<0.01)$ or in combination with PA $(p<0.01)$. Also EPA upregulated $m f n 2$ relative to PA alone $(p<0.05)$ confirming enhanced mitochondrial fusion and therefore function. However, OA, alone or in combination with PA, increased intracellular Oil Red $\mathrm{O}$ staining, indicating a shift in lipid metabolism toward triglyceride synthesis $(p<0.001)$. To 
conclude, EPA and OA decrease the effects of PA by two distinct mechanisms: EPA promotes mitochondrial fusion which increases fatty acid utilisation, while OA channels excess PA toward triglyceride synthesis. Thus, EPA and OA can decrease ceramide synthesis and hence lipotoxicity via separate mechanisms.

\subsection{The Effect of Human Adenovirus-36 on Lipid Metabolism in a Rat Model}

Fatemeh Shirani ${ }^{1}$, Ali Teimoori ${ }^{2}$, Mohammad Rashno ${ }^{3}$, Seyed Mahmoud Latifi ${ }^{4}$ and Majid Karandish ${ }^{5, *}$

1 Student Research Committee, Ahvaz Jundishapur University of Medical Sciences, Ahvaz, Iran

2 Infectious and Tropical Diseases Research Center, Ahvaz Jundishapur University of Medical Sciences, Ahvaz, Iran

3 Department of Immunology, School of Medicine, Ahvaz Jundishapur University of Medical Sciences, Ahvaz, Iran

4 Diabetes Research Center, Ahvaz Jundishapur University of Medical Sciences, Ahvaz, Iran

5 Nutrition and Metabolic Diseases Research Center, Ahvaz Jundishapur University of Medical Sciences, Ahvaz, Iran

* Corresponding Author

Obesity is a multifactorial disease that is a major global health challenge. A positive correlation between obesity and several pathogens, with a particular emphasis on the human adenovirus-36 (Ad36) has been shown in animal models and it is considered as a possible risk factor for obesity in humans. Ad-36 may increase adipose tissue with paradoxical reduction of serum lipids in animal models; the results, however, were not consistent. The present study aimed to investigate the relationship between Ad-36 infection and lipid metabolism in a rat model. Sixty male Wistar rats (eight-week-old) were used for the experiment. Blood samples were collected at the beginning of the study and 3 months later in both infected (48 rats) and control groups (12 rats). The mean \pm standard deviation (SD) changes observed in serum triglycerides $(-15.3 \pm 22.4$ vs. $0.1 \pm 17.9 \mathrm{mg} / \mathrm{dL}, p=0.027)$, low-density lipoprotein cholesterol $(-1.5 \pm 4.0$ vs. $1.1 \pm 2.2 \mathrm{mg} / \mathrm{dL}, p=0.008)$, and total serum cholesterol $(-8.4 \pm 10.6$ vs. $1.5 \pm 5.3 \mathrm{mg} / \mathrm{dL}, p=0.001)$ were significantly lower in the infected group compared with the control group. There were no significant differences in high-density lipoprotein cholesterol ( $1.4 \pm 7.2$ vs. $-2.7 \pm 7.2 \mathrm{mg} / \mathrm{dL}, p=0.09)$ levels between the infected and control groups. We find a statistically significant association between Ad-36 infection and lipid metabolism. The present study shows some differences to the previous rat model study. Rats can be used as a good animal model for further investigations about Ad-36-induced obesity.

\subsection{Appropriate Gestational Weight Gain for Chinese Women}

J. Lai, J. Wang, Z. Yang, S. Yin, Y. Duan, X. Pang, S. Jiang and Y. Bi

National Institute for Nutrition and Health, Chinese Center for Disease Control and Prevention, Beijing 100050, China

Gestational weight gain (GWG) is a key clinical indicator for the health of mother and child, but no GWG guidelines are available for Chinese women. In total, 8323 mothers with full-term singleton babies at $0 \sim 24$ months postpartum were investigated from Chinese National Nutrition and Health Surveillance in 2013. GWG (by subtracting the prepregnancy weight from the weight before delivery), postpartum weight retention (PWR, by subtracting the prepregnancy weight from the postpartum weight), pregnant outcomes and child growth zscores were collected and calculated. The $P 50(P 25 \sim P 75)$ of GWG was 15.0(10.0 19.0) kg, and the proportions of inadequte, appropriate and excessive GWG accounted for $27.2 \%(2263 / 8323), 36.2 \%(3016 / 8323)$ and $36.6 \%(3044 / 8323)$ according to IOM GWG guidelines. Excessive GWG increased the risk of hypertension $(2.6 \% \& 1.6 \%, p=0.03)$, cesarean section $(55.7 \% \& 42.3 \%, p<0.001)$, macrosomia $(14.3 \% \& 9.0 \%, p<0.001)$, overweight or obesity of children $(16.9 \% \& 12.0 \%, p=0.003)$, and high PWR $(49.9 \% \& 30.5 \%, p<0.001)$, but inadequate GWG increased the risk of stunting $(9.9 \% \& 7.0 \%, p=0.006)$ compared to appropriate GWG group. Mothers with good pregnant outcomes, low PWR, and good growth status of their 
children were identified, and they were divided into four groups according to their prepregnancy BMI $\left(<18.5 \mathrm{~kg} / \mathrm{m}^{2}, 18.5 \sim 23.9 \mathrm{~kg} / \mathrm{m}^{2}, 24.0 \sim<27.9 \mathrm{~kg} / \mathrm{m}^{2}\right.$ and $\left.\geq 28.0 \mathrm{~kg} / \mathrm{m}^{2}\right)$; the optimal GWG ranges for each group were $(11.5 \sim 18.0) \mathrm{kg}$, (10.0 15.0) kg, (8.0 14.0) kg and (5.0 11.5) kg. The GWG guidelines based on Chinese women and BMI categories may be used to give clinical recommendations for promoting pregnant outcomes, lower PWR and optimal child growth.

\subsection{Contextualizing Food Experiences of New Skilled Migrant Mothers from an Emerging Community in Australia and Post-Migration Risk for Obesity}

F. Kavian, K. Mehta, J. Coveney and L. Matwiejczyk

Flinders University, College of Nursing and Health Sciences, Adelaide 5042, Australia

Despite acknowledgement of the importance of food to health, well-being and identity, there is a dearth of research into the migrant experience, particularly for skilled-migrants from new ethnic minorities in Australia. The role of nutrition is especially important in certain 'lifestyle' diseases such as obesity that impact disproportionately these populations in Australia. This qualitative study attempted to understand the perceptions and experiences about food and health of new Iranian female migrants who are family food providers. The research explored contextual factors in food acculturation when adjusting and managing food provisioning at the early stages of transition. Seven in-depth interviews using a semi-structure questionnaire were conducted with mothers aged 25 to 43 years, living in Adelaide, who had immigrated to Australia within the past 5 years. Four main themes emerged: food meanings and identity; maintaining traditional food ways; sociocultural pressures associated with migration and the role of children in acculturation. The findings reveal that migration to Australia appeared to bring new stresses in relation to employment and changes to domestic roles for women. Participants reported the incorporation of new western foods, usually at the request of their children which deviates from healthier traditional food ways. This research highlights the importance of psychosocial factors and politico-economic context in shaping migrant food experiences and how the process of adaptation might introduce potential risks for obesity in this population. Nutrition practitioners and public health providers may use these findings to support and develop strategies to improve advocacy during the adjustment experiences and prevent obesity.

\subsection{Lifestyle Intervention Strategies That Target Weight Outcomes in People with Psychosis; a Systematic} Review

D. W. Mucheru 1, M. C. Hanlon 1,2, M. McEvoy ${ }^{3,4}$ and L. MacDonald-Wicks 1,5

1 The University of Newcastle, Callaghan, Australia

2 Brain and Mental Health Program, Hunter Medical Research Institute, New Lambton, Australia

3 School of Medicine and Public Health, Faculty of Health and Medicine, The University of Newcastle, Callaghan, Australia

4 Centre for Clinical Epidemiology and Biostatistics, Hunter Medical Research Institute, New Lambton, Australia

5 Priority Research Centre for Physical Activity and Nutrition, The University of Newcastle, Callaghan, Australia

The aim of this study was to systematically review the efficacy of different lifestyle intervention strategies on weight outcomes in people with psychosis. This systematic review considered for inclusion randomised controlled studies that delivered lifestyle interventions targeting weight outcomes (weight, body mass index, waist circumference and waist-to-hip ratio) in communitydwelling adults with a psychotic disorder. We aimed to find studies published in English from the Cochrane Library, MEDLINE/PREMEDLINE, EMBASE, CINAHL, Scopus, and PsycINFO. We included 29 papers after screening and all lifestyle intervention studies provided nutrition and or physical activity components as the main form of intervention. Delivery of nutrition and physical activity information was mainly through education, using strategies including motivational interviewing, cognitive behavior therapy and psychoeducation. In addition to education, some studies enforced personalization of the intervention by helping participants set goals specific to 
nutrition or physical activity or both and provided review of progress on a regular basis. These studies reported statistically significant between group differences in weight outcomes more frequently than studies that did not personalize intervention components or offer regular review. Studies that did not offer personalization of intervention components or offer regular review but reported statistically significant differences in weight outcomes tended to have additional intervention components such as financial incentives or psychotherapy, which may have had a positive impact on outcomes. Lifestyle interventions offering nutrition and physical activity education for people with psychosis should personalize education by providing assistance with goal setting and offer regular review to attain significant weight loss results.

\title{
3.160. Fortified Natural Goat Milk Retains Dairy Lipid Bioactives
}

E. Carpenter ${ }^{1}$, C. Prosser ${ }^{1}$, E.-L. Andersson ${ }^{2}$ and O. Hernell ${ }^{2}$

1 Dairy Goat Co-Operative (NZ) Ltd., Hamilton, New Zealand

2 Pediatrics, Department of Clinical Sciences, Umeå University, Umeå, Sweden

Human breast milk is the best choice to provide infant nutrition and the gold standard used when designing infant formula. The major proportion of energy contained in breast milk is derived from milk fats. However, few current infant formulae contain milk lipids as it has become common to discard milk fat and the majority of the Milk Fat Globule Membrane (MFGM) during manufacture. Recently, clinical studies have supported the hypothesis that milk fat and MFGM plays a beneficial role in infant nutrition. The MFGM is a three-layered membrane, containing lipids and proteins originating from the secretory cell membrane, surrounding the milk fat globule triglyceride core. The MFGM contains numerous lipids (such as sphingomyelin and gangliosides), sialic acid, and cholesterol, but also a small $(\sim 2 \%)$ proportion of the total milk protein, including bioactive components such as mucin (MUC1), butyrophilin and lactadherin. Infant formula made by fortifying goat milk, that retains natural milk lipids and MFGM through the manufacturing process, has been examined to profile the milk-fat derived bioactives that are retained in powdered product following formulation. Further, fat blends made by combining selected vegetable oils with the cream fraction of goat milk were subjected to lipolysis under experimental conditions resembling the small intestine of newborn infants. Our results confirm that inclusion of goat milk fat in the fat blend for infant formula had no detrimental effect on lipolysis and retains important functional components from milk fat.

\subsection{Consumption Pattern of Malay Herbal Medicines during Pregnancy and Postpartum among} Malaysian Mothers

\author{
A. B. Normina ${ }^{1}$, T. Noradhiah ${ }^{1}$, C. K. Tan ${ }^{1}$, Y. B. Ho ${ }^{2}$, M. S. Seghayat ${ }^{1}$ and E. S. S. Tan ${ }^{1}$ \\ 1 Faculty of Medicine and Health Sciences, UCSI University, Malaysia \\ 2 Faculty of Medicine and Health Sciences, Universiti Putra Malaysia, Malaysia
}

Herbal medicine is increasingly in-demand as an alternative medicine. They had been consumed for generations to improve one's well-being. In Malaysia, Malay herbal medicines (MHMs) are commonly consumed during pregnancy and postpartum by mothers of the Malay community. This study aims to determine respondents' demographics, pattern of MHMs consumption as well as pregnancy and neonatal characteristics. This is a comparative questionnaire study involving 106 postpartum mothers; consisting of 64 consumers and 42 non consumers. Median age for respondents was 31 and ranged from 22 to 44 years old. Respondents' household income was $\leq$ MYR5,000 (52.8\%, $\mathrm{n}=56)$ and $>$ MYR5,000 $(47.2 \%, \mathrm{n}=50)$ and was significantly associated $(p<0.05)$ with consumption of MHMs. No association was found between consumption of MHMs with education, occupation and personal income. MHMs consumption during pregnancy and confinement were $20.3 \%$ and $50 \%$ respectively. The top three MHMs consumed were Ubat Periuk $(12.5 \%, \mathrm{n}=8)$, Manjakani $(26.6 \%, \mathrm{n}=$ $17)$ and Nona Roguy confinement set $(20.3 \%, n=13)$. Consumption of MHMs during pregnancy did not influence preterm delivery as well as neonatal birth weight, birth length and head circumference. 
Subsequently, the reported odds ratio for neonatal jaundice and need for phototherapy were 1.03 and 2.78 respectively. Meanwhile, mothers who did not consume MHMs during pregnancy reported lower incidences $(p<0.05)$ and lower risk $(\mathrm{OR}=0.25)$ of threatened abortions. Higher risk of neonatal jaundice $(\mathrm{OR}=1.24)$ was found for postpartum consumption. In conclusion, household income was an influencing demographic factor for MHMs consumption. Odds for phototherapy nearly tripled with pregnancy consumption; non-consumption appeared to be protective against threatened abortion.

\subsection{Children's Unhealthy Food Intake: Application of a Behavioural Economics Lens to Understand Parental Decision-Making}

R. K. Golley ${ }^{1}$, E. Huynh ${ }^{2}$, B. J. Johnson ${ }^{1}$ and J. Swait ${ }^{2}$

1 Nutrition and Food Science, School of Pharmacy and Medical Sciences, University of South, Adelaide, SA 5000, Australia

2 Institute for Choice, School of Business, UniSA, Sydney, NSW 2060, Australia

Public health nutrition programs have been unsuccessful in changing children's intakes of unhealthy foods. To better design programs and campaigns, the study aim was to understand the decision-making processes that influence parents' unhealthy food provision to children. A randomly selected sample of 500 parents of 4-12-year-old children were recruited from an online consumer research panel. The DCE was administered via a 20-min online survey. Parents were presented with lunch and dinner meal options that varied in nutritional value and cost. Econometric analysis determined parental lunchbox and dinner provision preferences. Explanatory variables examined the goals and trade-offs that parents prioritised. Child's age, household income and postcode (metropolitan/non-metropolitan) suggest a broadly representative sample reflective of the Australian internet population. A multiple goal-directed choice model, examining nutritional value and cost simultaneously explained parental food provision preferences with good fit. The most preferred lunchbox had medium cost and high energy. The least preferred lunchbox was the most expensive and was also high energy. This study is unique in that it represents parental motives across two eating occasions. Future research will determine whether parental choices are amendable to change via intervention messages based on their decision-making processes.

\subsection{Fat-Free Mass of Preterm Infants Fed Higher-Protein Human Milk Fortifier}

J. Miller, C. Davis, B. Lingwood and C. Collins

University of Newcastle, Newcastle 2300, Australia

Preterm infants typically incur a protein deficit during their neonatal admission, leading to suboptimal growth and body composition. In a previous RCT of preterm infants during their neonatal admission (mean GA 27.4 weeks, mean birth weight $1034.1 \mathrm{~g}$ ), we compared growth of infants receiving human milk fortifier containing $1 \mathrm{~g}$ protein $/ 100 \mathrm{~mL}$ expressed breast milk (standard care) with growth of infants receiving fortifier containing additional $0.4 \mathrm{~g}$ protein $/ 100 \mathrm{~mL}$ expressed breast milk. We found no difference in weight or length gain between groups, but reduced growth faltering in the higher protein group. Bioelectrical impedance spectroscopy (BIS) was used to measure body composition, but this data was not analysed due to lack of a valid equation for predicting fat-free mass (FFM).

The present study developed and validated an equation for predicting FFM in a separate cohort of preterm infants (mean GA 32.0 weeks, mean birth weight $1782.0 \mathrm{~g}$ ) using multiple regression and Bland-Altman analyses. We used this equation to predict FFM in the RCT cohort, and then used linear mixed models to determine differences between groups in FFM change, and Independent samples Ttests to determine differences between groups in proportion of FFM at study end. Weight and L2/R50 were the most significant predictors of FFM and were therefore included in the FFM prediction equation. We found no differences in FFM change or proportion of FFM at study end between groups 
in the RCT cohort. We therefore postulate that a mechanism other than, or in addition to, protein deficit may mediate suboptimal FFM deposition in preterm infants.

\title{
3.164. Exploring the Role of Grandparents in the Feeding of Grandchildren Aged 1-5 Years
}

\author{
E. M. Rogers, L. K. Bell and K. P. Mehta
}

Nutrition and Dietetics, School of Health Sciences, Flinders University, Bedford Park, SA 5042, Australia

Early childhood is a crucial time for the development of eating behaviours and food preferences. As a result of increased labour force participation by Australian parents of young children, grandparents are now acting as the main informal carers of young children. Participation in the care of their grandchildren gives grandparents the capacity to influence young children's food-related behaviour. The aim of this study was to gain insight into the perspectives of grandparents regarding the feeding of their grandchild/ren aged 1-5 years old. Semi-structured interviews were conducted with 11 grandparents (nine grandmothers and two grandfathers) from suburban Adelaide in South Australia, to determine their beliefs and opinions regarding feeding their 1 to 5 year old grandchild/ren. Thematic analysis revealed four categories: (1) intergenerational differences; (2) maintaining family relationships; (3) treating grandchildren with food and (4) nutritional efficacy. These findings highlight that grandparents thoughtfully manage familial relations regarding the feeding of children, as a result of intergenerational differences in practices. They also show some paradoxical thinking whereby grandparents prioritise the provision of healthy food whilst also 'treating' their grandchildren with unhealthy foods. This is not atypical compared to the rest of the population. Grandparents' social role in the complex psycho-social space of children's feeding warrants serious recognition and deeper understanding, in order to fully engage them as key stakeholders in children's nutritional health and ultimately improve young children's eating behaviours.

\subsection{A Profile of Milk Consumption among Australian Children and Its Impact on Nutrient Intakes with a} Focus on Flavoured Milk

\section{F. Fayet-Moore ${ }^{1}$, A. McConnell ${ }^{1}$ and J. Kim ${ }^{2}$ \\ 1 Nutrition Research Australia, Sydney, NSW 2000, Australia \\ 2 Nestlé Australia Ltd., Rhodes, NSW 2138, Australia}

Research has shown that flavoured milk can assist children in meeting nutrient targets. We aimed to profile consumption of milk types and its impact on nutrient intakes. Day 1 data from the 2011-2012 National Nutrition and Physical Activity Survey for children 2-18 y were analysed (n = 2812). Children were classified as plain, flavoured or other milk drinkers (e.g., milkshakes, coffee), non-drinkers (e.g., cereal) and milk avoiders. Total daily milk and nutrients were calculated and adjusted for confounders using general linear models. In total, $81 \%$ of children consumed milk. A total of $36 \%$ of milk was consumed as non-beverages, and $64 \%$ as beverages: $34 \%$ plain, $19 \%$ flavoured, $11 \%$ other milk. Among milk consumers, $37 \%$ were non-drinkers and $63 \%$ drinkers: $29 \%$ drank plain, $20 \%$ flavoured, $13 \%$ other milk. The highest prevalence of flavoured milk drinkers was among $9-13$ y (24\% of milk consumers) and among $14-18$ y there were more flavoured (18\%) than plain milk drinkers (15\%). Flavoured milk drinkers consumed more daily milk (480 $\pm 10.7 \mathrm{~g})$ than other milk drinkers $(245 \pm 13.8 \mathrm{~g})$ and non-drinkers $(165 \pm 8.2 \mathrm{~g})(p<0.001)$, but the same as plain milk drinkers $(445 \pm 9.0 \mathrm{~g})$. Daily calcium intakes were higher among flavoured $(1049 \pm 18.2 \mathrm{mg})$ and plain milk drinkers $(980 \pm 15.2 \mathrm{mg}$ ) than other milk drinkers $(783 \pm 23.3 \mathrm{mg})$, non-drinkers $(718 \pm 13.8 \mathrm{mg})$, and avoiders ( $587 \pm 17.5 \mathrm{mg}$ ). There was no difference in daily calcium or free sugars between plain and flavoured milk drinkers. Flavoured and plain milk drinkers had similar nutrient profiles, and higher calcium intake than other children. Flavoured milk was most prevalent among 9-18 y who are most at risk of calcium inadequacies and offers an opportunity to increase milk and calcium intake. 


\subsection{Maternal Diet during Breastfeeding and Infant Acceptance of Foods during the Weaning Period}

\section{J. Joyce and M. A. Choukri}

Department of Nursing, Midwifery, and Allied Heath, Ara Institute of Canterbury, Christchurch, New Zealand

Food-related sensory experiences during infancy may lay the foundation of the development of healthy eating habits in early childhood and beyond. Infants who have been exposed to a wide variety of flavours in breast milk may be more accepting of a wider variety of foods during the weaning period. This study investigates if there is a relationship between maternal dietary intake during breastfeeding and acceptance of a variety of food flavours in six to eight month old breastfed infants. Thirty mothers of infants have been recruited to date to participate in this study. Mothers complete a FFQ questionnaire including common fruits and vegetables, cereals and meats for the breastfeeding period, and infant food acceptance during the weaning period assessed as a number of times a food has been offered to the infant before it was accepted. Regression analyses will be used to compare the maternal frequency of food consumption during breastfeeding with the preparedness of the infant to accept the food. Secondary analyses will investigate the relationship of an overall variety in maternal diet and infant food acceptance or refusal. Findings from this study are expected to show that repeated exposure to flavours in mother's milk enhances acceptance of the same flavours for the infant, and a varied maternal diet increases infant food acceptance during the weaning period.

\subsection{Promoting Recommended Baby Feeding Practices to Improve the Nutritional Status of Young Children in Jogjakarta, Indonesia}

M Louisa ${ }^{1}$, L Matwiejczyk ${ }^{1}$, N. Kuntolowati ${ }^{2}$, R. Kaeksi ${ }^{3}$, Miftakhuljannah ${ }^{4}$ and D. C. Sulistyoningrum ${ }^{5}$

1 Discipline of Nutrition and Dietetics, College of Nursing and Health Sciences, Flinders University, Adelaide, SA, Australia

2 Ngemplak 2 Community Health Centre (Puskesmas Ngemplak 2), Sleman, Indonesia

3 Tempel 1 Community Health Centre (Puskesmas Tempel 1), Sleman, Indonesia

4 Mlati 2 Community Health Centre (Puskesmas Mlati 2), Sleman, Indonesia

5 Department of Nutrition and Health, Faculty of Medicine Universitas Gadjah Mada, Yogyakarta, Indonesia

In Yogyakarta, Indonesia, malnutrition in very young children is a significant public health problem, with stunting affecting optimal physical and cognitive development and school performance. It is also associated with a higher risk of morbidity and mortality and permanent consequences in later adult life. In rural Indonesia, the prevalence of malnutrition and its associated forms continues at concerning rates. Breast feeding rates can improve childhood nutrition and help mitigate this issue. Data, however, suggests that the scope of exclusive breastfeeding is below national targets. In addition, observations have shown that recommended baby feeding practices issued by the central and local government in villages are not necessarily known or followed by all mothers. A significant barrier to breastfeeding is the support and attitude of fathers. This study describes a novel program which aimed to increase the number of babies and children aged under two years who are exclusively breastfed and optimally fed through the development of a breastfeeding fathers support group. Methods: In the rural villages of Ngemplak in Sleman, Yogyakarta, fathers of breast-feeding mothers developed a support group for men between December-April 2017 with guidance from the local nutritionist and Head of Sub-District and supported the mothers with feeding practices and practicalities. Results: Process evaluation objectives were met and preliminary feedback was very positive, prompting more qualitative investigation. Conclusion: Data on breastfeeding rates and practices is being collected. Fathers' attitudes and support are a significant determinant of breastfeeding rates and qualitative research is needed to further understand fathers' motivations for participation. 
3.168. Personalized Nutrition Kit Development to Determine Secretor Status of Chinese Mothers by

Detecting a Specific Single Nucleotide Polymorphism (rs1047781)

X. Ze, Y. Wang, C. Deng and L. Chen

Biostime Institute of Nutrition and Care, Guangzhou, China

Background: The purpose of this pilot study was to develop an easy, convenient and noninvasive sample collection kit for Chinese mothers to allow personalized detection for secretor status and precise prediction for human milk oligosaccharides (HMO) composition in their milk. Methods: Secretor status was determined by detecting the genotype of the SNP site rs1047781 of the FUT2 (2'fucosyltransferase) gene, using the DNA extracted from the oral cells collected from an oral cell collecting kit developed in this study. Breast milk was also collected separately from those mothers for HMO (2'-fucosyllactose, (2'-FL), as a representative) detection, using high performance anion ion exchange Chromatography, to investigate the association between genotype and phenotype. Results: The result showed that the kit allowed sufficient biological sample collection and delivery at room temperature. The DNA extracted was of good quality for subsequent gene detection. Seventeen of the total 22 mothers were secretors with the genotype AA $(n=7)$ or AT $(n=10)$ while the other five mothers were non-secretors, with the genotype TT. Twelve mothers were selected for HMO detection. The 2'-FL concentrations in milk of secretor mothers were AA, $1.70 \pm 0.86 \mathrm{~g} / \mathrm{L}(\mathrm{n}=4), \mathrm{AT}$, $0.56 \pm 0.17 \mathrm{~g} / \mathrm{L}(\mathrm{n}=4)$, respectively. While the 2 '-FL concentration was under the detection limit in the milk of non-secretor mothers $(n=4)$. Conclusion: This pilot study confirmed that the genotype of SNP rs1047781 can be used as an indicator for HMO composition. Our work provides mothers an easy, reliable and realizable tool for personalized gene detection for milk nutrition.

\subsection{Ruth Marcia English AO-Her Life's Work and Legacy in Public Health Nutrition}

B. Wood

\section{Affiliation not provided}

Ruth English (1929-2015), distinguished Public Health Nutritionist and Dietitian, worked tirelessly in the development of public health nutrition (PHN) policies and strategies in Australia and internationally. Biographical information was gathered by direct participation in some of these national PHN activities, also literature review and discussion with colleagues. Ruth English left Australia (1954) to work in England and the United States. This early work and post-graduate study experience in America were to establish her career path and the basis of her subsequent professional achievements in Australia and internationally. Returning to Australia (1957), Ruth was employed as a Nutritionist in the Commonwealth Department of Health, and while her children were young she worked there on an intermittent basis. In 1979, she was appointed Chief Nutritionist for Australia and immediately began rapid development of the Department's Nutrition Section and her prodigious professional output. It was a time of growing maturity and awareness of the role of food and nutrition in population health, and the need to consult and co-ordinate the views of many experts. On resignation from the public service (1993), her professional activities continued as nutrition consultant for Australian organizations and many regional governments in Far East Asia and Oceania. The chronological content of her biography demonstrates an exceptional era of activity in PHN which has not been fully sustained since. Through initiation of many different PHN policies and strategies, Ruth English conducted her life's work and left an inspiring legacy in public health nutrition.

\subsection{Indonesian Independently Practicing Midwives' Response towards Pregnant Women Nutrition Android Application: A Qualitative Study}

D. N. Chandra ${ }^{1}$, S. Bardosono ${ }^{1}$, Aryati ${ }^{2}$ and E. Wasito ${ }^{2}$

1 Nutrition Department Faculty of Medicine Universitas Indonesia-Cipto Mangunkusumo General Hospital, West Java 16424, Indonesia

2 Danone Nutricia Early Life Nutrition, Macquarie Park 2113, Australia. 
We aimed to explore factors contributing to midwives' response towards a new android application (the Apps) developed by digitalizing Indonesian Ministry of Health's book of Mother and Child Health with additional nutritional status analysis of the pregnant women. One hundred and sixty five independently practicing midwives from four selected cities (Medan, Jakarta, Semarang and Malang) were trained to use the Apps, i.e., to record at least 10 pregnant women's health data in their daily practice and diagnose their nutritional status using the Apps within three months. Evaluation was conducted for the Apps usage based on the health record data uploaded by the midwives which were classified as poor user ( $\leq 3$ data/user), average user (4-6 data/user), and frequent user ( $\geq 7$ data/user). Respondents of this qualitative study were chosen with the purposive sampling method based on extreme cases. In-depth interviews were conducted on 24 midwives, i.e., 17 midwives classified as poor users and 7 midwives classified as frequent users. Data collection and analysis were carried out by two investigators by using the triangulation approach. Data was coded and analyzed using the strength-weakness-opportunity-threat (SWOT) method. Almost all respondents have good response towards the Apps. The midwives consider the Apps to be useful with main challenge concerning the time issues. The amount of time allocated for using the Apps was considered as an additional work time since they also have to make reports for the health ministry and the midwife association. Therefore, the Apps require enhancement in providing downloadable reports needed by the midwives to increase the usage.

\subsection{A Sustainable and Collaborative Approach to Integrating Indigenous Health Content within Nutrition Science Curricula}

\section{J. McCartan, J. Dart, A. Dordevic and A. Bryce}

Department of Nutrition, Dietetics and Food, Monash University, Melbourne, Australia

There is increasing recognition for the need to embed cultural competency curricula in tertiary education for nutrition professionals. In 2014, the Australian Department of Health released the Aboriginal and Torres Strait Islander Health Curriculum Framework which aims to prepare graduates to practice in a culturally safe manner with Indigenous communities. In 2017, an Indigenous curriculum working party was established within the Department of Nutrition, Dietetics and Food at Monash University. The purpose of this paper is to describe the preliminary steps undertaken to scaffold and integrate Indigenous content across a three-year nutrition science program. Indigenous content has been largely concentrated and taught in distinct units. The working party is progressing a two-year plan to map, evaluate and highlight strengths, gaps and opportunities for developing teaching, learning and assessment approaches to support fulfilment of the 17 learning outcomes articulated in the framework from novice to entry level. This process has enabled an interdisciplinary collaboration within the academic team, with guidance from the faculty Indigenous engagement unit, to explore opportunities to integrate innovative Indigenous content across the curricula. Diverse expertise of the academic team include public health nutrition, nutrition science, dietetics, nutrigenomics and humanities. This has enabled embedding Indigenous content across the program with a broad focus, including professionalism, history, food and culture, nutrition science, health across the lifespan, sports nutrition, food and sustainability and public health nutrition. This paper provides a working example of the collaborative approach required to integrate Indigenous health content in a meaningful way within nutrition science curricula.

\subsection{Preparedness of Nutrition Science Students to Address Future Food and Sustainability Challenges}

\section{J. McCartan, L. Barbour and M. Bonham}

Department of Nutrition, Dietetics and Food, Monash University, Melbourne, Australia

Whole of system, multi-sectorial responses are required to address the environmental consequences of industrialised food production practices. Nutrition scientists can play a key role in recognizing opportunities to improve human and environmental health outcomes within a food supply system approach. To date, there have been limited tertiary-based opportunities to prepare the 
future nutrition science workforce to create healthy and sustainable food systems. In response to this need, Monash University introduced a single semester, compulsory undergraduate food sustainability unit, which is now in its second year. The purpose of this study is to assess student satisfaction with this unit and their preparedness to address the sustainability challenges posed throughout semester. Unit evaluation comprised an online survey implemented at the commencement of semester ( $55 \%$ response rate) and end of semester ( $39 \%$ response rate). Both surveys focused on student interest and confidence to pursue a future career in the field. Evaluation results revealed that the majority of students believed that the unit had added value to their studies in nutrition science (score 82/100) and that knowledge gained through the unit would be useful in their future career (score 70/100). Additionally, students reported an increase in confidence to pursue a future career in the field of food and sustainability (score 48/100 pre-semester compared to 70/100 post-semester). This demonstrates that embedding a food sustainability unit within existing nutrition science curricula has the capacity to positively influence Australia's future nutrition workforce by improving their preparedness to address future food and sustainability challenges.

\subsection{Causal Loop Diagram of Structural Contributors to the Japanese Healthy Diet}

\section{N. Nishi}

National Institutes of Biomedical Innovation, Health and Nutrition, Tokyo, Japan

The life expectancy of the Japanese is one of the highest in the world, which is attributable, in part, to a healthy diet and a relatively low proportion of overweight and obesity among the population. However, Japan faces many challenges such as a rapidly increasing proportion of elderly population and accumulating national debt. This study aimed to examine structural contributors to a balanced diet (taking meals with staple food, main dish and side dish at least twice a day) using a causal loop diagram of system dynamics. Included in the model were health-related variables (proportion of people with balanced diet, proportion of people with normal weight, life expectancy, health literacy of the people), demographic variables (proportion of elderly population, proportion of youth population, average age of the first marriage, birth rate), political variables (conservativeness of the people), economic variables (national medical expenditure, social security cost, national debt, gross domestic product, industrial advancement, household budget), educational variables (university advancement rate, subsidy for national university, tuition fee for university, household education expense), agriculture-related variables (restructure of agriculture, subsidy for agriculture), and food-related variables (import of food, household food expense, variety of available food, unit price of staple food). A total of 14 loops was identified on "proportion of people with balanced diet", and there were four reinforcing loops and 10 balancing loops. The model indicates that dynamically complex structural variables are affecting each other to contribute to the Japanese healthy diet and long life expectancy. (This work was supported by KAKENHI 15K08761.)

\subsection{Reflection of Clinical Instructor's towards Dietetic Placement for Nutrition Students}

Harry F. Luglio ${ }^{1}$, Susetyowati ${ }^{1}$, Toto Sudargo ${ }^{1}$, Amanda Wray ${ }^{2}$ and Jacqueline Miller ${ }^{2}$

1 Department of Health and Nutrition, Faculty of Medicine, Universitas Gadjah Mada, Farmako street, Sekip Utara Yogyakarta 55281, Indonesia

2 Nutrition and Dietetics, Flinders Medical Centre, Flinders University, Adelaide, SA 5042, Australia

(1) Background: Indonesia is currently improving the dietetic education system by introducing a dietetic internship for nutrition students during their fourth year of undergraduate study. To facilitate this, Universitas Gadjah Mada (UGM) collaborated with Flinders University, who hosted an Australian Award Fellowship program focused on training for clinical instructors in Indonesia. Fifteen academics and clinical instructors attended the program in Adelaide in February 2017. On return to Indonesia, UGM conducted training for 17 local clinical instructors. The objective of this study is to report the initial reflections from the clinical instructors on dietetic placement within their institution; (2) Methods: Clinical instructors were asked to evaluate the learning process and students 
performance during clinical placements using the following five questions: "What has been done correctly during the dietetic placement?", "What has not been done correctly during the dietetic placement?", "What have you learnt about the clinical practice?", "Do you need specific training for trainers?"; (3) Results: Key issues for clinical instructors were the students' attitude during dietetic placement. The majority of the clinical instructors agreed that they need specific training to improve their skills and knowledge in guiding students; (4) Conclusion: Several areas should be improved during dietetic placement so that the Dietetic Internship Program can be improved.

(C) 2018 by the authors. Licensee MDPI, Basel, Switzerland. This article is an open access article distributed under the terms and conditions of the Creative Commons Attribution (CC BY) license (http://creativecommons.org/licenses/by/4.0/). 